\author{
Universidade de São Paulo \\ Instituto de Física
}

\title{
Existência de Pares " $d$-wave" e Ondas de Densidade em uma Classe de Modelos Microscópicos para Supercondutores com Alta Temperatura de Transição
}

\author{
Antonio Delgado De Pasquale
}

Orientador: Prof. Dr. Walter de Siqueira Pedra Coorientador: Prof. Dr. Jean-Bernard Bru

Tese de doutorado apresentada ao Instituto de Física da Universidade de São Paulo, como requisito parcial para a obtenção do título de Doutor em Ciências.

Banca Examinadora:

Prof. Dr. Walter de Siqueira Pedra - Orientador (IFUSP)

Prof. Dr. Domingos Humberto Urbano Marchetti (IFUSP)

Prof. Dr. Gustavo Barbagallo de Oliveira (UFMG)

Prof. Dr. Silas Luiz de Carvalho (UFMG)

Prof. Dr. Roberto Venegeroles Nascimento (UFABC)

São Paulo 


\section{Agradecimentos}

Agradeço principalmente a meu orientador Walter Pedra e meu co-orientador J.-B. Bru pelo constante apoio e paciência no desenvolvimento deste trabalho, pela valiosa guia neste avance significativo de meu conhecimento no campo da física matemática aplicada na matéria condensada. Também agradeço ao IFUSP e o BCAM pelo ambiente do estudo facilitado, e a CNPq pelo importante apoio financeiro. 


\section{Resumo}

Os supercondutores de alta temperatura crítica apresentam propriedades diferentes dos supercondutores convencionais, uma destas propriedades importantes é a simetria não isotrópica do parâmetro de ordem. Neste trabalho se apresenta um modelo que mostra a presença de simetria $d$-wave em uma classe de supercondutores de alta $T_{c}$, assim como a condição para a existência de ondas de densidade nos estados de equilíbrio. Se analisa primeiro um sistema de três corpos, um bipolaron e dois elétrons, com o objetivo de estudar o efeito da repulsão coulombiana local e não local sobre a simetria do parâmetro de ordem para o caso exato e também o limite de acoplamento forte. Se mostra também a construção de um Hamiltoniano efetivo que desacopla a interação elétron-bipolaron com o fim de abordar o problema coletivo de infinitos corpos e vemos como o modelo proposto prediz a formação de ondas de densidade para uma região especifica dos parâmetros físicos do Hamiltoniano estudado. 


\begin{abstract}
High-temperature superconductors have different properties than conventional superconductors, one of these important properties is non-isotropic symmetry of the order parameter. In this work we present a model that shows the presence of symmetry $d$-wave in a class of superconductors of high $T_{c}$, as well as the condition for the existence of density waves in the states of equilibrium. We first analyze a three-body system, a bipolaron and two electrons, in order to study the effect of local and non-local Coulomb repulsion on the symmetry of the order parameter for the exact case and also the strong coupling limit. It is also shown the construction of an effective Hamiltonian that decouples the electron-bipolaron interaction in order to approach the collective problem of infinite bodies and we see how the proposed model predicts the formation of density waves for a specific region of the physical parameters of the studied Hamiltonian .
\end{abstract}




\section{Conteúdo}

1 Integral Direta de Espaços de Hilbert $\quad 11$

1.1 Espaço Mensurável e Integral de Lebesgue . . . . . . . . . . . . . . . . . . 12

1.1.1 Espaço Mensurável . . . . . . . . . . . . . . . . . . . . . . . . . . 12

1.1.2 Medida de Dirac e Função Característica . . . . . . . . . . . . . . . 12

1.1.3 Integral de Lebesgue . . . . . . . . . . . . . . . . . . . . . 13

1.2 Espaço de Hilbert $L^{2}\left(X, d \mu ; \mathcal{H}^{\prime}\right) \ldots \ldots \ldots \ldots$

1.3 Integral Direta de Fibras de Operadores . . . . . . . . . . . . . 15

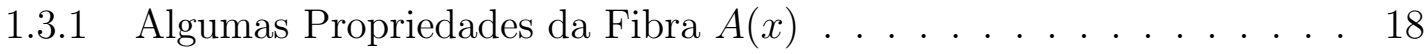

2 Um Modelo Microscópico para Cupratos Supercondutores 27

2.1 Modelo Bipolaron-Elétron para Supercondutores de Alta $T_{c} \ldots \ldots$. . . . . 28

2.1.1 Espaço de Dois Férmions e um Bóson . . . . . . . . . . . . . . 33

2.1.2 Decomposição em Fibras do Hamiltoniano Unitariamente Equivalente a $H^{(2,1)} \ldots \ldots \ldots \ldots \ldots$

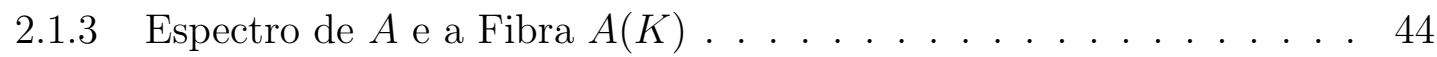

2.1.4 Estabilidade da Formação de Pares de Férmions . . . . . . . . . . . 54

2.1.5 Existência de Simetria $d$-wave no Parâmetro de Ordem . . . . . . . 55

3 Efeitos do Alcance da Repulsão Eletrônica e da Interação Bipolaron$\begin{array}{ll}\text { Elétron sobre a Simetria dos Pares } & 61\end{array}$

3.1 Estudo Numérico de Termos de Repulsão não Locais . . . . . . . . . . . . . 62

3.2 Considerações para o Calculo das Componentes de Função de Onda e Simetria $p$-wave . . . . . . . . . . . . . . . . . . . . . 65 
4 Ajuste do Modelo Bipolaron-Elétron para o Caso do $\mathrm{La}_{2} \mathrm{CuO}_{4} \quad 71$

4.1 Parâmetros Prototípicos dos Experimentos . . . . . . . . . . . . . . . . 74

4.2 Consequências fenomenológicas . . . . . . . . . . . . . . 77

4.3 Simetrias na Função de Onda dos Pares . . . . . . . . . . . . . . . . . . . . 82

5 Modelo Fermiônico Efetivo e Ondas de Densidade $\quad 91$

5.1 Hamiltoniano Fermiônico Efetivo . . . . . . . . . . . . . . . . . . . . 92

5.2 Cálculo da Pressão Termodinâmica . . . . . . . . . . . . . . . . . 97

$5.2 .1 \quad$ Caso $d$-wave . . . . . . . . . . . . . . . . . . . . . . . . . . . 99

5.2 .2 Caso $p$-wave . . . . . . . . . . . . . . . . . . . . . . 103

5.3 Condição no Limite de Baixas Temperaturas para Existência de Ondas de Densidade no Caso $d$-wave e $p$-wave . . . . . . . . . . . . . . 106

5.4 Ondas de Densidade . . . . . . . . . . . . . . . . . . . . . 109

5.4.1 Outra Condição para a Existência de Ondas de Densidade . . . . . 113

5.5 Efeito do Termo Cinético na Pressão Termodinâmica . . . . . . . . . . . . 119 


\section{Introdução}

Cupratos e outros materiais supercondutores com alta temperatura crítica tem propriedades que não são usuais, comparadas ás propriedades dos supercondutores convencionais ou BCS. Algumas destas propriedades exóticas, como por exemplo a simetria $d$-wave no parâmetro de ordem, são comuns em supercondutores de alta $T_{c}$, em particular os chamados cupratos [1]. O estudo de um modelo microscópico geral para o tipo típico desta classe de materiais pode revelar mecanismos que expliquem as propriedades observadas nestes supercondutores.

De fato, os princípios microscópicos da supercondutividade de alta $T_{c}$ são ainda objeto de debate. O objetivo deste trabalho é estudar rigorosamente um modelo microscópico que explique a presença da simetria $d$-wave observada em alguns supercondutores não típicos, assim como a formação de ondas de densidade nos estados de equilíbrio. Na primeira etapa apresentada neste trabalho se analisa o problema de três corpos, dois férmions e um bóson, interagindo em uma rede bidimensional perfeita. Rigorosamente se mostra como o modelo proposto conduz a uma simetria $d$-wave na função fermiônica que descreve o par de elétrons acoplados.

Heuristicamente para a função de onda $\psi$ do par de elétrons, temos

$$
\psi\left(r_{\uparrow}-r_{\downarrow}\right)=\sum_{k} g(k) \mathrm{e}^{i k \cdot\left(r_{\uparrow}-r_{\downarrow}\right)}
$$

onde $r_{\uparrow}$ e $r_{\downarrow}$ são respetivamente as posições associadas ao elétron "up" e "down". Por conveniência, discutiremos antes a transformada de Fourier $g(k)$ que a função de onda $\psi\left(r_{\uparrow}-r_{\downarrow}\right)$ diretamente. A função $g(k)$ é isotrópica no caso do supercondutores típicos ou 
"BCS". Se diz que tais supercondutores apresentam "supercondutividade $s$-wave". Como se mencionou anteriormente, nos supercondutores de alta $T_{c}$ é observada a presença de supercondutividade $d$-wave, ou seja a função $g(k)$ não é isotrópica e presenta inversão de sinal sob rotações de $90^{\circ}$ no espaço de momento, ou seja

$$
g\left(k_{x}, k_{y}\right)=-g\left(k_{y},-k_{x}\right)
$$

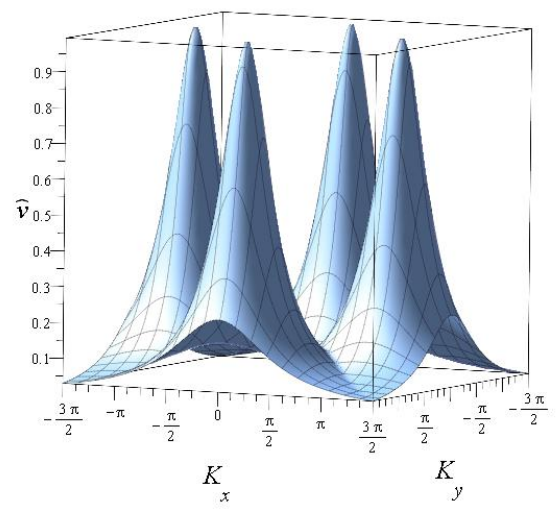

Figura 1: Função acoplamento $\hat{v}$.

Com base em fatos experimentais [2] foi proposto que nos supercondutores de alta $T_{c}$ a interação atrativa entre dois elétrons é mediada por um bipolaron (um par ligado, espacialmente localizado, de elétrons interagindo mediante fônons). O termo da interação entre elétrons e bipolarons proposto é

$$
W^{(\kappa)} \doteq \sum_{x, y \in \mathbb{Z}^{2}} v(x-y)\left(b_{x}^{\dagger} c_{y}^{(\kappa)}+c_{y}^{(\kappa) \dagger} b_{x}\right)
$$

onde

$$
c_{x}^{(\kappa)} \doteq \sum_{z \in \mathbb{Z}^{2},|z| \leq \gamma \kappa^{-1}} \mathrm{e}^{-\kappa|z|} a_{x+z, \uparrow} a_{x, \downarrow}
$$

é o operador de destruição de um par eletrônico e $b_{x}$ o operador de destruição de um bipolaron, com $x \in \mathbb{Z}^{2}$. O parâmetro $\gamma \in \mathbb{R}^{+}$é fixo (tipicamente $\gamma \sim 1$ ) e $\gamma \kappa^{-1} \in \mathbb{R}^{+}$ quantifica o "tamanho do bipolaron".

Mostramos rigorosamente que o modelo de interação bipolaron-elétron conduz a uma simetria $d$-wave, para estados de energia mínima, que depende fortemente de que a função de acoplamento $(\hat{v})$ presente picos no espaço de momento em $( \pm \pi, 0),(0, \pm \pi)$ tal como é observado na figura acima. Deslocando os picos desta função mostramos também que 
uma componente da função do par de elétrons tem simetria $p$-wave, ou seja

$$
g\left(k_{x}, k_{y}\right)=-g\left(-k_{y},-k_{x}\right)
$$

No primeiro capitulo introduzimos algumas ferramentas que vão a ser usadas para o estudo do problema dos três corpos, em particular a integral direta de espaços de Hilbert e a decomposição em fibras de operadores autoadjuntos. No seguinte capítulo se estuda em detalhe o problema com o Hamiltoniano proposto, que é o Hamiltoniano de Hubbard mais a interação bipolaron-elétron, e se considera em geral o caso de repulsão não local. Para o caso de repulsão local, que é possível tratar de forma analítica, se encontra o ponto mínimo do espectro do Hamiltoniano fibra e se consegue mostrar a presença de simetria $d$-wave no estado fundamental. Depois se analisa o caso não local com auxílio de cálculos numéricos. Com o propósito de testar nosso modelo para o caso particular do $\mathrm{La}_{2} \mathrm{CuO}_{4}$ fizemos também os ajustes nos parâmetros do Hamiltoniano para conseguir aproximar-nos dos dados experimentais para este material. Neste caso se parametrizo de forma independente a repulsão coulombiana local e não local e para a interação bipolaron-elétron se considerou uma estrutura mais próxima aos dados experimentais para o caso particular do $\mathrm{La}_{2} \mathrm{CuO}_{4}$. Finalmente se discute como é possível desacoplar as interações bipolaron-elétron em um Hamiltoniano efetivo puramente fermiônico, com o fim de analisar o problema coletivo de infinitos corpos e mostrar as condições para a formação de ondas de densidade nos estados de equilíbrio.

Para a analise do problema de infinitos corpos (principalmente o cálculo do limite termodinâmico da pressão) o termo de interação efetivo foi tratado mediante o método do Hamiltoniano aproximado de Bogoliubov [3]. A simetria do Hamiltoniano aproximado permite deduzir propriedades dos estados de equilíbrio, em particular como eles podem ser escritos como estados produto. Usando esta simetria e o método do Bogoliubov se calcula o limite termodinâmico da pressão de forma similar como é feito em [4]. Mostramos que existe uma região de parâmetros onde o problema variacional de Bogolioubov associado, apresenta duas soluções. Nesta região de parâmetros temos então uma família de soluções para as quais a "pressão local" oscila na rede $\mathbb{Z}^{2}$. Calculamos assim a densidade eletrônica 
local para este tipo de soluções, mostrando que nosso modelo prediz a formação de ondas de densidade em uma família de estados de equilíbrio.

A aproximação de campo médio é crucial na analise do problema de infinitos corpos. Mostramos como é "natural" definir no modelo efetivo o operador de interação $\tilde{W}^{\prime}$ de campo médio para $\Lambda \subset \mathbb{Z}^{2}$

$$
\tilde{W}^{\prime}:=-\frac{1}{|\Lambda|} \sum_{x, y \in|\Lambda|} w(x-y) c_{x}^{\dagger} c_{y}=-\hat{w}|\Lambda| \sum_{k_{m}}\left(\frac{1}{|\Lambda|} \sum_{x \in \Lambda} \mathrm{e}^{i k_{m} \cdot x} c_{x}\right)^{\dagger}\left(\frac{1}{|\Lambda|} \sum_{x \in \Lambda} \mathrm{e}^{i k_{m} \cdot x} c_{x}\right)
$$

Como é provado em [4], os estados de equilíbrio para um ponto da rede vem dados por a dinâmica do Hamiltoniano aproximado de Bogoliubov com as constantes $\mathfrak{b}_{m}$ (5.6) que atingem a pressão de equilíbrio. Assim temos os estados de Bogoliubov dados por

$$
\Omega_{\eta, \tilde{\eta}}(\cdot)=\frac{\operatorname{Tr}\left(\cdot \mathrm{e}^{-\beta H_{\Lambda_{b}}}\right)}{\operatorname{Tr}\left(\mathrm{e}^{-\beta H_{\Lambda_{b}}}\right)}
$$

Vemos que o estado $\Omega_{\eta, \tilde{\eta}}(\cdot)$ atua sobre elementos $A \in \mathcal{U}_{\Lambda_{b}}$, onde $\mathcal{U}_{\Lambda_{b}}$ é a álgebra $C^{*}$ gerada por $\left\{a_{x, \uparrow}, a_{x, \downarrow}, \mathbb{1}\right\}$ com $x \in \Lambda$. Notamos também que o estado $\Omega_{\eta, \tilde{\eta}}(\cdot)$ é um estado produto, ou seja se $A_{i} \in \mathcal{U}_{i} \operatorname{com} \mathcal{U}_{i}$ a sub-álgebra $C^{*}$ local gerada por $\left\{a_{i, \uparrow}, a_{i, \downarrow}, \mathbb{1}\right\}$ com $i \in \mathbb{Z}^{2}$, e assim

$$
\Omega_{\eta, \tilde{\eta}}\left(\prod_{i} A_{i}\right)=\prod_{i} \Omega^{i}\left(A_{i}\right)
$$

com

$$
\Omega^{i}(\cdot)=\frac{\operatorname{Tr}\left(\cdot \mathrm{e}^{-\beta H_{i}}\right)}{\operatorname{Tr}\left(\mathrm{e}^{-\beta H_{i}}\right)} .
$$

Calculamos, a partir dos estados de Bogoliubov, a densidade eletrônica por site da rede e a existência de estados de equilíbrio com ondas de densidade. Depois disto mostramos que a presença de um termo cinético com um parâmetro de "hopping" pequeno não modifica a "estrutura" da pressão termodinâmica de equilíbrio e portanto a presença das ondas de densidade nos possíveis estados de equilíbrio. 


\section{Capítulo 1}

\section{Integral Direta de Espaços de}

\section{Hilbert}

Existe uma classe de espaços de Hilbert, cujos elementos (os espaços) podem ser interpretados como uma "soma contínua" de espaços de Hilbert. Tendo um espaço de Hilbert $\mathcal{H}^{\prime}$ podemos encontrar outro espaço de Hilbert $\mathcal{H}$ que seja isomórfico a uma "soma direta continua" de $\mathcal{H}^{\prime}$, simbolicamente

$$
\mathcal{H} \cong \int_{X}^{\oplus} \mathcal{H}^{\prime} d \mu
$$

onde $X$ é um espaço mensurável e $\mu$ uma medida.

Um subconjunto dos operadores limitados que vão de $\mathcal{H}$ em $\mathcal{H}$ podem interpretar-se também como uma "soma contínua de operadores" $A^{\prime}$, onde cada um atua sobre o espaço $\mathcal{H}^{\prime}$. Simbolicamente

$$
A \cong \int_{X}^{\oplus} A^{\prime} d \mu
$$

O espaço $\mathcal{H}^{\prime}$ é chamado espaço fibra ou fibra, assim como $A^{\prime}$ é chamado a fibra do operador $A$. No caso de que $A$ seja autoadjunto, os espectros dos operadores $A$ e $A^{\prime}$ estão vinculados de uma forma simples, assim é possível encontrar propriedades entre os espectros da fibra e o operador $A$.

Com o objetivo de fazer uma introdução ao tema se mencionará brevemente as 
definições de espaço mensurável e integral de Lebesgue.

\section{Espaço Mensurável e Integral de Lebesgue}

\section{Espaço Mensurável}

Seja a tripla

$$
(X, \Sigma, \mu)
$$

onde

- $X$ é um conjunto qualquer

- $\Sigma$ é uma $\sigma$-álgebra, uma coleção de subconjuntos de $X$ tal que:

$$
\begin{aligned}
& -\emptyset, X \in \Sigma \\
& -A \in X \Rightarrow X \backslash A \in \Sigma \\
& \text { - } \bigcup_{n=1}^{\infty} A_{n} \in X \text { quando } A_{n} \in X
\end{aligned}
$$

- $\mu$ é uma medida, $\mu: \Sigma \rightarrow \mathbb{R}_{0}^{+}$tal que:

$$
\begin{aligned}
& -\mu(\emptyset)=0 \\
& -\mu\left(\bigcup_{n=1}^{\infty} A_{n}\right)=\sum_{n=1}^{\infty} \mu\left(A_{n}\right) \quad \text { se } A_{n} \cap A_{m}=\emptyset \forall n \neq m .
\end{aligned}
$$

A tripla $(X, \Sigma, \mu)$ é chamada espaço de medida.

\section{Medida de Dirac e Função Característica}

Seja $X \neq \emptyset x \in X$ e $A \in \Sigma$, a medida de Dirac se define por

$$
\delta_{x}(A)=\left\{\begin{array}{lll}
1 & \text { se } & x \in A \\
0 & \text { se } & x \notin A
\end{array}\right.
$$


Dado um conjunto $A$ mensurável contido em $X$, se define a função característica

$$
\chi_{A}(x)=\left\{\begin{array}{lll}
1 & \text { si } & x \in A \\
0 & \text { si } & x \notin A
\end{array}\right.
$$

\section{Integral de Lebesgue}

$\underline{\text { Função Simples }}$

Uma função $f: X \rightarrow \mathbb{R}$ é chamada simples se seu range é finito, isto é, se

$$
f=\sum_{j=1}^{n} \alpha_{j} \chi_{A_{j}} \quad \text { con } \quad A_{j}=f^{-1}\left(\alpha_{j}\right) \in \Sigma
$$

Por definição,

$$
\int_{X} \chi_{A}(x) d \mu=\mu(A)
$$

Para uma função simples $\left(f=\sum_{j=1}^{n} \alpha_{j} \chi_{A j}\right)$,

$$
\int_{X} f d \mu=\sum_{j=1}^{n} \alpha_{j} \int_{X} \chi_{A_{j}} d \mu=\sum_{j=1}^{n} \alpha_{j} \mu\left(A_{j}\right)
$$

e para uma função $f$ não simples positiva,

$$
\int_{X} f d \mu=\sup _{s \leq f} \int_{X} s d \mu
$$

Para $f$ positiva $\int_{X} f d \mu=0 \Rightarrow f=0$ a.e. (onde a. e. significa "almost everywere", fora de um conjunto de medida zero) 


\section{Espaço de Hilbert $L^{2}\left(X, d \mu ; \mathcal{H}^{\prime}\right)$}

Seja $(X, \mu)$ um espaço mensurável e $\mathcal{H}^{\prime}$ um espaço de Hilbert. Seja $L^{2}\left(X, d \mu ; \mathcal{H}^{\prime}\right)$ o conjunto de funções mensuráveis sobre $X$ com valores em $\mathcal{H}^{\prime}$, que satisfazem

$$
\int_{X}\|f(x)\|_{\mathcal{H}^{\prime}}^{2} d \mu<\infty
$$

O conjunto é um espaço de Hilbert com o produto interno

$$
\langle f, g\rangle=\int_{X}\langle f(x), g(x)\rangle_{\mathcal{H}^{\prime}} d \mu
$$

Notemos o que acontece se $\mu$ é a soma de

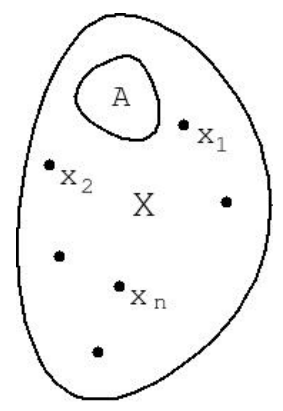

Figura 1.1: Ilustração do conjunto $X$ e os pontos $x_{i} \in X$ associados as medidas de Dirac. medidas de Dirac sobre um número finito de pontos $\left\{x_{1}, x_{2}, \cdots, x_{n}\right\}$

$$
\mu(A)=\sum_{i=1}^{n} \delta_{x_{i}}(A) \quad A \in \Sigma
$$

$\mu(X)$ será finita e igual a $n$. Para uma função simples $\phi=\sum_{k=1}^{l} \alpha_{k} \chi_{A_{k}} \operatorname{com} A_{k}=\phi^{-1}\left(\alpha_{k}\right)$ temos

$$
\begin{gathered}
\int_{X} \phi d \mu=\sum_{k=1}^{l} \alpha_{k} \mu\left(A_{k}\right)=\sum_{i, k} \alpha_{k} \delta_{x_{i}}\left(A_{k}\right)=\sum_{i, k} \alpha_{k} \chi_{A_{k}}\left(x_{i}\right) \\
=\sum_{i=1}^{n} \phi\left(x_{i}\right)
\end{gathered}
$$

então,

$$
\int_{X}\|f(x)\|_{\mathcal{H}^{\prime}}^{2} d \mu=\sum_{i=1}^{n}\left\|f\left(x_{i}\right)\right\|_{\mathcal{H}^{\prime}}^{2} .
$$

Com esta medida discreta cada $f \in L^{2}\left(X, d \mu ; \mathcal{H}^{\prime}\right)$ fica determinada pela $n$-tupla 
$\left(f\left(x_{1}\right), f\left(x_{2}\right), \cdots, f\left(x_{n}\right)\right)$, assim o espaço $L^{2}\left(X, d \mu ; \mathcal{H}^{\prime}\right)$ é isomorfo a

$$
\bigoplus_{i=1}^{n} \mathcal{H}^{\prime}
$$

e mais concretamente

$$
\begin{aligned}
\varphi: L^{2}\left(X, d \mu, \mathcal{H}^{\prime}\right) & \rightarrow \bigoplus_{i=1}^{n} \mathcal{H}^{\prime} \quad f \mapsto\left(f\left(x_{1}\right), f\left(x_{2}\right), \cdots, f\left(x_{n}\right)\right) \\
\langle f, g\rangle_{L^{2}\left(X, d \mu ; \mathcal{H}^{\prime}\right)} & =\int_{X}\langle f(x), g(x)\rangle_{\mathcal{H}^{\prime}} d \mu=\sum_{i=1}^{n}\left\langle f\left(x_{i}\right), g\left(x_{i}\right)\right\rangle_{\mathcal{H}^{\prime}} \\
& =\langle\varphi(f), \varphi(g)\rangle_{\oplus_{i=1}^{n} \mathcal{H}^{\prime}} .
\end{aligned}
$$

Vemos então que com uma medida discreta $L^{2}\left(X, d \mu ; \mathcal{H}^{\prime}\right) \cong \bigoplus \mathcal{H}^{\prime}$. Para una medida não discreta $\mathcal{H}=L^{2}\left(X, d \mu ; \mathcal{H}^{\prime}\right)$ será isomorfo a uma "especie de soma continua" de espaços $\mathcal{H}^{\prime}$, então se escreve simbolicamente

$$
\mathcal{H}=\int_{X}^{\oplus} \mathcal{H}^{\prime} d \mu
$$

$\mathcal{H}$ é chamado integral direta de espaços de Hilbert ou integral direta de fibras constantes, onde a fibra é o espaço $\mathcal{H}^{\prime}$.

\section{Integral Direta de Fibras de Operadores}

Agora vamos ver o espaço de os operadores lineares e acotados $B(\mathcal{H}): \mathcal{H} \rightarrow \mathcal{H}$ $\left(\mathcal{H}=\int_{X}^{\oplus} \mathcal{H}^{\prime} d \mu=L^{2}\left(X, d \mu ; \mathcal{H}^{\prime}\right)\right)$.

Seja $A \in B(\mathcal{H})$

$$
A \psi=\psi^{\prime} \quad \psi, \psi^{\prime} \in \mathcal{H}
$$

se

$$
A \psi(x)=\psi^{\prime}(x)=A(x) \psi(x) \quad \psi^{\prime}(x) \in \mathcal{H}^{\prime} \quad A(x) \in B\left(\mathcal{H}^{\prime}\right) \text { para } x \text { a.e. }
$$


se diz que A é a integral direta das fibras $A(x)$ e se escreve

$$
A=\int_{X}^{\oplus} A(x) d \mu
$$

Vemos que se $A$ pode ser descomposto em fibras, existe associado a este operador uma função mensurável $A(\cdot): X \rightarrow B\left(\mathcal{H}^{\prime}\right)$.

Agora vamos ver a que espaço pertence a função $A(\cdot)$. Se nós pensamos primeiro em uma função $a(\cdot) \in L^{\infty}(X, \mu ; \mathbb{C})$, quer dizer uma função que vai aos complexos e que é acotada em nos conjuntos cuja medida é diferente de zero ou essencialmente acotada

$$
\|a(\cdot)\|_{\infty}=\operatorname{ess} \sup |a(x)|<\infty
$$

podemos multiplicar $a(x)$ pelo operador identidade do espaço $B\left(\mathcal{H}^{\prime}\right)$, assim $a(x) I_{B\left(\mathcal{H}^{\prime}\right)}$ define um operador $A: \mathcal{H} \rightarrow \mathcal{H}$

$$
A \psi(x)=a(x) I_{B\left(\mathcal{H}^{\prime}\right)} \psi(x)
$$

Pode ser visto que $A \in B(\mathcal{H})$

$$
\begin{gathered}
\int_{X}\|A \psi(x)\|_{\mathcal{H}^{\prime}}^{2} d \mu=\int_{X}\|a(x) \psi(x)\|_{\mathcal{H}^{\prime}}^{2} d \mu \\
\|A \psi\|_{\mathcal{H}}^{2} \leq \operatorname{ess} \sup |a(m)|^{2}\|\psi\|_{\mathcal{H}}^{2} .
\end{gathered}
$$

Justamente pode ser provado que

$$
\|A\|_{B(\mathcal{H})}=\operatorname{ess} \sup |a(m)|=\|a(\cdot)\|_{\infty}
$$

isto quer dizer que ainda cada $a(\cdot) \in L^{\infty}(X, \mu ; \mathbb{C})$ define um operador $A \in B(\mathcal{H})$, também existe uma isometria entre estes espaços $\left(B(\mathcal{H})\right.$ e $\left.L^{\infty}(X, \mu ; \mathbb{C})\right)$, só que estritamente $L^{\infty}(X, \mu ; \mathbb{C})$ seria isométrico a um subespaço de $B(\mathcal{H})$ que vamos chamar $B_{m}(\mathcal{H})$, aqueles do tipo multiplicativo

$$
B_{m}(\mathcal{H}) \cong L^{\infty}(X, \mu ; \mathbb{C}) \otimes \mathbb{I}_{B\left(\mathcal{H}^{\prime}\right)}
$$


O caso restrito anterior pode dar a ideia para uma isometria geral que cubra tudo o espaço de operadores que podem ser descompostos em fibras. Seja uma função mensurável $A(\cdot): X \rightarrow B\left(\mathcal{H}^{\prime}\right)$, tal que

$$
A \Psi(x)=A(x) \Psi(x)
$$

Como vimos antes, a condição principal para encontrar a isometria entre os espaços era o requerimento da limitação essencial. Agora vamos pedir que $\|A(\cdot)\|_{B\left(\mathcal{H}^{\prime}\right)}$ seja essencialmente limitado

$$
\text { ess } \sup \|A(\cdot)\|_{B\left(\mathcal{H}^{\prime}\right)}<\infty
$$

assim, seguindo os mesmos passos que no caso restrito obtemos que

$$
\sup \frac{\|A \Psi\|_{\mathcal{H}}}{\|\Psi\|_{\mathcal{H}}}=\|A\|_{B(\mathcal{H})}=\operatorname{ess} \sup \|A(\cdot)\| \|_{B\left(\mathcal{H}^{\prime}\right)}
$$

como ess $\sup \|A(\cdot)\|_{B\left(\mathcal{H}^{\prime}\right)}$ é justamente $\|A(\cdot)\|_{\infty}, A(\cdot) \in L^{\infty}\left(X, \mu ; B\left(\mathcal{H}^{\prime}\right)\right)$, então

$$
\|A\|_{B(\mathcal{H})}=\|A\|_{\infty}
$$

Novamente vemos que para cada $A(\cdot) \in L^{\infty}\left(X, \mu ; B\left(\mathcal{H}^{\prime}\right)\right)$, existe um $A \in B(\mathcal{H})$ que pode ser descomposto em fibras, então chamando $B_{d}(\mathcal{H})$ a este espaço temos que

$$
B_{d}(\mathcal{H}) \cong L^{\infty}\left(X, \mu ; B\left(\mathcal{H}^{\prime}\right)\right)
$$

Vemos então que a cada operador $A \in B_{d}(\mathcal{H})$ é associado a uma função $A(\cdot) \in$ $L^{\infty}\left(X, \mu ; B\left(\mathcal{H}^{\prime}\right)\right)$, mas $A(\cdot)$ define um continuo de operadores, daí a notação heurística

$$
A=\int_{X}^{\oplus} A(x) d \mu
$$

ainda como $\|A\|_{B(\mathcal{H})}=\|A(\cdot)\|_{\infty}, A=0 \Rightarrow A(\cdot)=0$ a.e., o que lembra a propriedade das integrais de funções positivas $\int f d \mu=0 \Rightarrow f=0$ a.e.. 


\section{Algumas Propriedades da Fibra $A(x)$}

Seja $A \in B(\mathcal{H})$ e $A=A^{\dagger}$, temos que $(A+i)^{-1}$ existe e é limitado, ou seja $(A+i)^{-1} \in B(\mathcal{H})$.

Em particular se $\mathcal{H}=\int_{X}^{\oplus} \mathcal{H}^{\prime} d \mu$ e $A \in B_{d}(\mathcal{H}),(A+i)^{-1} \in B_{d}(\mathcal{H})$.

Para demostrar a afirmação acima suponhamos $z=x+i y$, então

$$
\begin{aligned}
\|(A+z) \Psi\|^{2}=\|(A+x) \Psi+i y \Psi\|^{2} & =\langle(A+x) \Psi+i y \Psi,(A+x) \Psi+i y \Psi\rangle \\
& =\quad\|(A+x) \Psi\|^{2}+y^{2}\|\Psi\|^{2}
\end{aligned}
$$

devido a que

$$
\langle(A+x) \Psi, i y \Psi\rangle=-\langle i y \Psi,(A+x) \Psi\rangle
$$

então vemos

$$
\begin{aligned}
\|(A+z) \Psi\|^{2} & =\|(A+x) \Psi\|^{2}+y^{2}\|\Psi\|^{2} \geq y^{2}\|\Psi\|^{2} \\
& \Rightarrow \operatorname{ker}(A+z)=\{0\} \Rightarrow(A+z)^{-1} \exists
\end{aligned}
$$

se colocamos $\Psi=(A+z)^{-1} \psi$

$$
\begin{array}{rlc}
\left\|(A+z)(A+z)^{-1} \psi\right\| & \geq & y^{2}\left\|(A+z)^{-1} \psi\right\|^{2} \\
& \Rightarrow & \|\psi\|^{2} \geq y^{2}\left\|(A+z)^{-1} \psi\right\|^{2} \\
& \Rightarrow & \frac{\left\|(A+z)^{-1} \psi\right\|}{\|\psi\|} \leq|y|^{-1} \\
& \Rightarrow\left\|(A+z)^{-1}\right\| \leq|y|^{-1} \Rightarrow \operatorname{com} z=i\left\|(A+i)^{-1}\right\| \leq 1
\end{array}
$$

Se $A \in B_{d}(\mathcal{H})$ temos então que $(A+i)^{-1} \in B_{d}(\mathcal{H})$, ou seja para $\Psi \in \mathcal{H}=\int_{X}^{\oplus} \mathcal{H}^{\prime} d \mu$,

$$
(A+i) \Psi(x)=(A(x)+i) \Psi(x)
$$


e

$$
(A+i)^{-1}=\int_{X}^{\oplus}(A(x)+i)^{-1} d \mu
$$

A propriedade anterior é importante para mostrar uma similar para grupos unitários gerados por operadores descomponíveis. Seja $A \in B_{d}(\mathcal{H})$ e autoadjunto, então

$$
\mathrm{e}^{i A t}=\lim _{n \rightarrow \infty}\left(\mathbb{1}-\frac{i A t}{n}\right)^{-n}
$$

devido a que o produto de dois operadores que podem ser decompostos em fibras também pode ser decomposto em fibras, ou seja para $T$ e $U$ pertencentes a $B_{d}(\mathcal{H})$ se tem que

$$
T U \Psi(x)=T(x) U(x) \Psi(m) \text { com } \Psi \in \mathcal{H}
$$

$\mathrm{Ou}$

$$
T U=\int_{X}^{\oplus} T(x) U(x) d \mu
$$

e com a propriedade mostrada acima, $\left\|(A+i)^{-1}\right\| \leq 1$, temos que $\mathrm{e}^{i A t} \in B_{d}(\mathcal{H})$

$$
\lim _{n \rightarrow \infty}\left(\mathbb{1}-\frac{i A t}{n}\right)^{-n}=\int_{X}^{\oplus} \lim _{n \rightarrow \infty}\left(\mathbb{1}-\frac{i A(x) t}{n}\right)^{-n} d \mu
$$

$\mathrm{Ou}$

$$
\mathrm{e}^{i A t}=\int_{X}^{\oplus} \mathrm{e}^{i A(x) t} d \mu
$$

O resultado anterior é usado para mostrar outra importante propriedade. Seja $\hat{f}(k)$ uma função real pertencente a espaço $L^{1}(\mathbb{R}), \int_{R}|f(k)| d k<\infty$, então

$$
f(x)=\int \hat{f}(k) \mathrm{e}^{i k x} d k
$$

e para um operador $A$ a imagem homomórfica da função $f(x)$ no espaço dos operadores será

$$
f(A)=\int \hat{f}(k) \mathrm{e}^{i k A} d k
$$


se $A \in B_{d}(\mathcal{H})$ e $A$ é autoadjunto temos que

$$
\begin{aligned}
f(A) & =\int \hat{f}(k)\left(\int_{X}^{\oplus} \mathrm{e}^{i k A(x)} d \mu\right) d k \\
& =\int_{x}^{\oplus} \int \hat{f}(k) \mathrm{e}^{i k A(x)} d k d \mu
\end{aligned}
$$

e

$$
f(A)=\int_{X}^{\oplus} f(A(x)) d \mu
$$

A propriedade acima é importante para estudar a relação entre o espectro da fibra $A(x)$ e o operador $A$. Com este fim temos que estudar primeiro um lema conhecido como "lema de Weyl".

Lema 1. Se existe uma sequência $\left\{\Psi_{n}\right\}$ pertencente a dominio do operador $A$ tal que $\left\|\Psi_{n}\right\|=1$ e $\left\|(A-\lambda) \Psi_{n}\right\| \rightarrow 0$ então $\lambda \in \sigma(A)$, onde $\sigma(A)$ é o espectro de A. A sequência $\left\{\Psi_{n}\right\}$ é chamada sequência de Weyl.

Demonstração. Seja $\left\{\Psi_{n}\right\}$ uma sequência de Weyl, então $\lambda \in \rho(A)$, onde $\rho(A)=\mathbb{C} \backslash \sigma(A)$, é impossível porque

$$
1=\left\|\Psi_{n}\right\|=\left\|(A-\lambda)^{-1}(A-\lambda) \Psi_{n}\right\| \leq\left\|(A-\lambda)^{-1}\right\|\left\|(A-\lambda) \Psi_{n}\right\| \rightarrow 0 .
$$




\section{Projeção Espectral}

Como é sabido o espaço de operadores limitados $B(\mathcal{H}), A: \mathcal{H} \rightarrow \mathcal{H}$, onde $H$ é um espaço de Hilbert, formam uma álgebra $C^{*}$, ou seja formam um espaço de Banach com as propriedades:

- $\|A B\| \leq\|A\|\|B\|$

- $\left(A^{*}\right)^{*}=A$

- $(A+B)^{*}=A^{*}+B^{*}$

- $(\alpha A)^{*}=\bar{\alpha} A^{*}$

- $(A B)^{*}=B^{*} A^{*}$

- $\|A\|^{2}=\left\|A^{*} A\right\| \quad \operatorname{com} A$ e $B \in B(\mathcal{H})$

onde a involução $*: B(\mathcal{H}) \rightarrow B(\mathcal{H})$ é a operação de tomar o operador adjunto.

Outro exemplo de álgebra $C^{*}$ é o espaço de funções essencialmente acotadas $L^{\infty}([a, b] ; \mathbb{R}) f:[a, b] \rightarrow \mathbb{R},[a, b] \subset \mathbb{R}$ e $\|f\|=\operatorname{ess} \sup |f(x)|$, onde a involução é a operação de tomar o conjugado complexo ponto a ponto. Quando as funções são reais $\bar{f}=f$, o que lembra a propriedade dos operadores autoadjuntos no espaço $B(\mathcal{H})$. De fato existe um homomorfismo, chamado homomorfismo $*$, entre a subálgebra $C^{*}$ de $B(\mathcal{H})$ dos operadores autoadjuntos e a álgebra $C^{*}$ de as funções reais essencialmente limitadas, definido por

$$
\phi: L^{\infty}([a, b] ; \mathbb{R}) \rightarrow B(\mathcal{H}) \quad \phi(f)=f(A)
$$

com

- $\phi(f g)=\phi(f) \phi(g)$

- $\phi(\lambda f)=\lambda \phi(f)$

- $\phi(1)=\mathbb{1}$

- $\phi(\bar{f})=\phi^{*}(f)$ 
Numa visão mais geral pode ser provado que todas as álgebras $C^{*}$ são isomórficas * a uma subálgebra $C^{*}$ de uma álgebra de operadores limitados $B(\mathcal{H})$ sobre um espaço de Hilbert $\mathcal{H}$, este fato é conhecido como Teorema de Gelfand-Naimark.

Agora queremos ver a imagem homomórfica $*$, entre $L^{\infty}([a, b] ; \mathbb{R})$ e os operadores autoadjuntos que pertencem a $B(H)$, para a função característica

$$
\chi_{\Omega}(x)=\left\{\begin{array}{lll}
1 & \text { si } & x \in \Omega \\
0 & \text { si } & x \notin \Omega
\end{array}\right.
$$

com $\Omega \subset \mathbb{R}$. Temos que $\phi\left(\chi_{\Omega}(\lambda)\right)=\chi_{\Omega}(A)$, o que a princípio não é trivial a reconhecer. Notando que

$$
\chi_{\Omega}^{2}(\lambda)=\chi_{\Omega}(\lambda) \Rightarrow \chi_{\Omega}^{2}(A)=\chi_{\Omega}(A)
$$

podemos inferir que $\chi_{\Omega}(A) \equiv P_{A}(\Omega)$ é um projetor ortogonal.

Temos também que

$$
\chi_{\Omega}(\lambda)=\sum_{i=1}^{n} \chi_{\Omega_{i}}(\lambda)
$$

para qualquer união finita $\Omega=\cup_{i=1}^{n} \Omega_{i}$ de conjuntos disjuntos. Vemos que

$$
\phi\left(\sum_{i=1}^{n} \chi_{\Omega_{i}}(\lambda)\right)=\sum_{i=1}^{n} \phi\left(\chi_{\Omega_{i}}(\lambda)\right)
$$

o que implica

$$
P_{A}(\Omega)=\sum_{i=1}^{n} P_{A}\left(\Omega_{i}\right)
$$

então

$$
P_{A}(\mathbb{R})=\mathbb{1} \quad P_{A}(\emptyset)=0 \quad P_{A}(R \backslash \Omega)=\mathbb{1}-P_{A}(\Omega) .
$$

Da propriedade

$$
\chi_{\Omega_{i}}(\lambda) \chi_{\Omega_{j}}(\lambda)=\chi_{\Omega_{i} \cap \Omega_{j}}(\lambda)
$$

temos também que

$$
P_{A}\left(\Omega_{i}\right) P_{A}\left(\Omega_{j}\right)=P_{A}\left(\Omega_{i} \cap \Omega_{j}\right)
$$


O conjunto $\left\{P_{A}\left(\Omega_{i}\right)\right\}$ de projetores é chamado família espectral. Para uma função simples $f=\sum_{i} \alpha_{i} \chi_{\Omega_{i}}, \Omega_{i}=f^{-1}\left(\alpha_{i}\right)$ temos

$$
\phi(f)=\sum_{i} \alpha_{i} \phi\left(\chi_{\Omega_{i}}\right)=\sum_{i} \alpha_{i} P_{A}\left(\Omega_{j}\right)
$$

do que segue que

$$
\phi(f)=\int_{\mathbb{R}} f(\lambda) d P_{\lambda}
$$

Disto vemos que a imagem homomórfica, nos operadores autoadjuntos pertencentes a $B(\mathcal{H})$, de qualquer função simples pode ser decomposta em projetores espectrais. Seja $\Psi$, $\varphi$ pertencentes a $\mathcal{H}$, vemos que

$$
\langle\varphi, \phi(f) \Psi\rangle=\sum_{i} \alpha_{i}\left\langle\varphi, P_{A}\left(\Omega_{i}\right) \Psi\right\rangle
$$

e $\mu_{\varphi, \Psi}\left(\Omega_{i}\right)=\left\langle\varphi, P_{A}\left(\Omega_{i}\right) \Psi\right\rangle$ tem as propriedades de uma medida, unicamente que com valores complexos em geral. Em termos desta medida, resulta que

$$
\langle\varphi, \phi(f) \Psi\rangle=\int_{\mathbb{R}} f(\lambda) d \mu_{\varphi, \Psi}
$$

Se $f(\lambda)=\lambda$ temos pela equação (1.2)

$$
A=\int_{\mathbb{R}} \lambda d P_{\lambda}
$$

Teorema 1. O espectro de A é dado por

$$
\sigma(A)=\left\{\lambda \in \mathbb{R} \mid P_{A}(\lambda-\epsilon, \lambda+\epsilon) \neq 0 \quad \forall \epsilon>0\right\}
$$

Demonstração. Seja $\Omega_{n}=\left(\lambda_{0}-\frac{1}{n}, \lambda_{0}+\frac{1}{n}\right)$, se $P_{A}\left(\Omega_{n}\right) \neq 0$ podemos encontrar um 
$\Psi_{n}=P_{A}\left(\Omega_{n}\right) \varphi \operatorname{com}\left\|\Psi_{n}\right\|=1$, como $\Psi_{n}=P_{A}\left(\Omega_{n}\right) \Psi_{n}$ temos que

$$
\begin{aligned}
\left\|\left(A-\lambda_{0}\right) \Psi_{n}\right\|^{2}=\left\|\left(A-\lambda_{0}\right) P_{A}\left(\Omega_{n}\right) \Psi_{n}\right\|^{2} & =\left\langle\left(A-\lambda_{0}\right) P_{A}\left(\Omega_{n}\right) \Psi_{n},\left(A-\lambda_{0}\right) P_{A}\left(\Omega_{n}\right) \Psi_{n}\right\rangle \\
& =\quad\left\langle\Psi_{n},\left(A-\lambda_{0}\right)^{2} P_{A}\left(\Omega_{n}\right) \Psi_{n}\right\rangle
\end{aligned}
$$

como

$$
\phi\left(\left(\lambda-\lambda_{0}\right)^{2} \chi_{\Omega_{n}}(\lambda)\right)=\left(A-\lambda_{0}\right)^{2} P_{A}\left(\Omega_{n}\right)
$$

temos que

$$
\left\langle\Psi_{n},\left(A-\lambda_{0}\right)^{2} P_{A}\left(\Omega_{n}\right) \Psi_{n}\right\rangle=\left\langle\Psi_{n}, \phi\left(\left(\lambda-\lambda_{0}\right)^{2} \chi_{\Omega_{n}}(\lambda)\right) \Psi_{n}\right\rangle
$$

e pela equação (1.3)

$$
\left\langle\Psi_{n}, \phi\left(\left(\lambda-\lambda_{0}\right)^{2} \chi_{\Omega_{n}}(\lambda)\right) \Psi_{n}\right\rangle=\int_{\mathbb{R}}\left(\lambda-\lambda_{0}\right)^{2} \chi_{\Omega_{n}} d \mu_{\Psi_{n}, \Psi_{n}} \leq \frac{1}{n^{2}} \int_{\mathbb{R}} \chi_{\Omega_{n}}(\lambda) d \mu_{\Psi_{n}, \Psi_{n}}
$$

dado que

$$
\frac{1}{n^{2}} \int_{\mathbb{R}} \chi_{\Omega_{n}}(\lambda) d \mu_{\Psi_{n}, \Psi_{n}}=\frac{1}{n^{2}} \mu_{\Psi_{n}, \Psi_{n}}\left(\Omega_{n}\right)=\frac{1}{n^{2}}\left\langle\Psi_{n}, P_{A}\left(\Omega_{n}\right) \Psi_{n}\right\rangle=\frac{1}{n^{2}}
$$

esto implica

$$
\left\|\left(A-\lambda_{0}\right) \Psi_{n}\right\|^{2} \leq \frac{1}{n^{2}}
$$

então encontramos uma sequência de Weyl, o que implica que $\lambda_{0} \in \sigma(A)$.

Reciprocamente, se $P_{A}\left(\lambda_{0}-\epsilon, \lambda_{0}+\epsilon\right)=0$ pode ver-se que $\lambda_{0} \in \rho(A)$. Seja

$$
f_{\epsilon}(\lambda)=\chi_{\mathbb{R} \backslash\left(\lambda_{0}-\epsilon, \lambda_{0}+\epsilon\right)}(\lambda)\left(\lambda-\lambda_{0}\right)^{-1}
$$

então

$$
\begin{aligned}
\left(A-\lambda_{0}\right) \phi\left(f_{\epsilon}(\lambda)\right)=\phi\left(\left(\lambda-\lambda_{0}\right) f_{\epsilon}(\lambda)\right) & =\quad P_{A}\left(\mathbb{R} \backslash\left(\lambda_{0}-\epsilon, \lambda_{0}+\epsilon\right)\right) \\
& =\mathbb{1}-P_{A}\left(\lambda_{0}-\epsilon, \lambda_{0}+\epsilon\right)=\mathbb{1} \Rightarrow \lambda_{0} \in \rho(A) .
\end{aligned}
$$


Em particular vemos que $P_{A}\left(\left(\lambda_{i}, \lambda_{j}\right)\right)=0$ unicamente se $\left(\lambda_{i}, \lambda_{j}\right) \subseteq \rho(A)$. Também $P_{A}(\sigma(A))=\mathbb{1}$ e $P_{A}(\mathbb{R} \cap \rho(A))=0$.

O Teorema anterior é importante para encontrar a conexão entre o espectro de um operador pertencente a $B_{d}(\mathcal{H})$ e o espectro da fibra $A(x)$. Da propriedade mostrada antes

$$
f(A)=\int_{X}^{\oplus} f(A(x)) d \mu
$$

temos que

$$
P_{A}(\lambda-\epsilon, \lambda+\epsilon)=\int_{X}^{\oplus} P_{A(x)}(\lambda-\epsilon, \lambda+\epsilon) d \mu
$$

lembrando que

$$
\|A\|_{B_{d}(\mathcal{H})}=\operatorname{ess} \sup \|A(x)\|_{B\left(\mathcal{H}^{\prime}\right)}
$$

temos

$$
A=0 \Rightarrow A(x)=0 \text { a.e. ou } A \neq 0 \Rightarrow A(x) \neq 0 \text { para } \quad x \in \mathrm{X} \text { onde } \mathrm{X}=\{x \mid \mu(x) \neq 0\}
$$

ou seja

$$
P_{A}(\lambda-\epsilon, \lambda+\epsilon)(A) \neq 0 \Rightarrow P_{A(x)}(\lambda-\epsilon, \lambda+\epsilon) \neq 0
$$

para algum $x$, ou subconjunto de $\mathrm{X}$, cuja medida não seja nula. Então $\lambda \in \sigma(A)$ unicamente se $\forall \epsilon>0$ existe ao menos um $x$ tal que $\mu(x)>0$ e

$$
\sigma(A(x)) \cap(\lambda-\epsilon, \lambda+\epsilon) \neq \emptyset
$$

$\mathrm{Ou}$

$$
\mu(\{x \mid \sigma(A(x)) \cap(\lambda-\epsilon, \lambda+\epsilon) \neq \emptyset\})>0
$$




\section{Capítulo 2}

\section{Um Modelo Microscópico para Cupratos Supercondutores}

Como é sabido alguns materiais, como os cupratos, apresentam supercondutividade em uma temperatura de transição alta (> $77 \mathrm{~K})$. Estes materiais são isolantes para temperaturas mais elevadas do que a temperatura crítica $T_{c}$, a diferença dos supercondutores de baixa $T_{c}$ que nas condições normais são condutores, explicados com sucesso pela teoria BCS. Outra propriedade importante é a simetria d-wave (observada experimentalmente) do parâmetro de ordem dos supercondutores de alta $T_{c}$, a diferença da simetria s-wave observada no os supercondutores BCS.

Um modelo que explica bem a transição de fase isolante para fase condutora (conhecida como transição de Mott) é o conhecido modelo de Hubbard [5]. O modelo de Hubbard toma no conta a repulsão coulombiana a diferença do modelo BCS. Normalmente é estudado o caso com repulsão local, onde os elétrons só experimentam a interação coulombiana quando são encontrados no mesmo site da rede. De fatos experimentais [2] tem sido proposto que nos supercondutores de alta $T_{c}$ a interação atrativa entre dois elétrons é mediada por um bipolaron (um par espacial de elétrons interagindo mediante fônons). Primeiro se fará um tratamento analítico, onde é mostrado a simetria do parâmetro de ordem, para o caso com repulsão local e depois um estudo numérico exato para o caso não local, onde não é possível fazer o cálculo de forma analítica. Com o fim 
de estudar a interação entre dois férmions (elétrons) mediada por um bóson (bipolaron) se considera só o caso de três partículas.

\section{Modelo Bipolaron-Elétron para Supercondutores de}

\section{Alta $T_{c}$}

De fatos experimentais e por simplicidade é considerado algumas suposições no modelo:

- Como foi explicitado antes, consideraremos em principio para o tratamento analítico só repulsão coulombiana local ou "repulsão de Hubbard".

- A interação entre as partículas são fortes comparada com a energia cinética (regime de acoplamento forte). Esta suposição é utilizada numa parte da analise mas é obtida a solução exata do problema para o caso de três corpos para qualquer ordem de magnitude da energia cinética.

- O movimento das partículas ocorre em uma rede bidimensional, isto devido a estrutura dos cupratos onde o material esta constituído por camadas que podem ser consideradas independentes.

- A interação entre elétrons e bipolarons é forte só no pontos bem localizados da primeira zona de Brillouin $(( \pm \pi, 0),(0, \pm \pi))[6,7]$.

- O spin total do bipolaron é zero.

- A energia cinética do bipolaron é pequena comparada com a dos elétrons, isto devido a que a massa dos bipolarons e bem maior que a massa dos elétrons.

- Um bipolaron pode decair em dois elétrons que por sua vez podem formar um bipolaron, sendo esta processo forte só nos pontos $( \pm \pi, 0),(0, \pm \pi)$.

- As interações são invariantes sob traslações, reflexões e rotações de $90^{\circ}$. 
Tomando em conta as suposições acima, propomos um Hamiltoniano para bósons e férmions na rede $\mathbb{Z}^{2}$. Consideramos também que o material é um cristal cubico bidimensional perfeito.

O espaço de Fock bosônico $(+)$ e fermiônico $(-)$ é dado por

$$
\mathcal{F}_{+-} \doteq \bigoplus_{n=0}^{\infty} \mathfrak{h}_{ \pm}^{n}
$$

onde $\mathfrak{h}_{ \pm}^{n}$ denota o $n$-ésimo, $n \in \mathbb{N}$, produto tensorial do espaço de um bóson $(+)$ e um férmion (-) $\left(\mathfrak{h}_{ \pm}^{1}\right)$. Lembrando que o espaço fermiônico (bosônico) de $n$ partículas é o subespaço simétrico (antissimétrico) do espaço de $n$ partículas. Por definição, o espaço vácuo $\mathfrak{h}_{-}^{0}=\mathfrak{h}_{+}^{0} \doteq \mathbb{C}$. O espaço do sistema composto de férmions e bósons é dado por

$$
\mathcal{F}_{-} \otimes \mathcal{F}_{+} \cong \bigoplus_{n, m=0}^{\infty} \mathfrak{h}_{-}^{m} \otimes \mathfrak{h}_{+}^{n}
$$

Em uma rede bidimensional $\left(\mathbb{Z}^{2}\right)$, o espaço de Hilbert para um elétron é dado por

$$
\mathfrak{h}_{-}^{1} \doteq \ell^{2}\left(\mathbb{Z}^{2} ; \mathbb{C}^{2}\right) \quad\left(\operatorname{spin} \frac{1}{2}\right)
$$

Como assumimos que os bipolarons tem spin total zero, o correspondente espaço de Hilbert para um bipolaron será

$$
\mathfrak{h}_{+}^{1} \doteq \ell^{2}\left(\mathbb{Z}^{2} ; \mathbb{C}\right) \quad(\operatorname{spin} 0)
$$

Os operadores de criação e destruição são denotados por

$$
a_{x, s}^{\dagger} \equiv a_{x, s}^{\dagger} \otimes \mathbf{1}_{\mathcal{F}_{+}}, \quad a_{x, s} \equiv a_{x, s} \otimes \mathbf{1}_{\mathcal{F}_{+}}, \quad x \in \mathbb{Z}^{2}, \quad s \in\{\uparrow, \downarrow\}
$$

para férmions, e

$$
b_{x}^{\dagger} \equiv \mathbf{1}_{\mathcal{F}_{-}} \otimes b_{x}^{\dagger}, \quad b_{x} \equiv \mathbf{1}_{\mathcal{F}_{-}} \otimes b_{x}, \quad x \in \mathbb{Z}^{2},
$$

para caso bosônico. 
A parte fermiônica do Hamiltoniano é definida por

$$
H_{f}^{(m)} \doteq \epsilon\left(-\frac{1}{2} \sum_{s \in\{\uparrow, \downarrow\}, x, y \in \mathbb{Z}^{2},|x-y|=1} a_{x, s}^{\dagger} a_{y, s}+2 \sum_{s \in\{\uparrow, \downarrow\}, x \in \mathbb{Z}^{2}} a_{x, s}^{\dagger} a_{x, s}\right)+\sum_{x \in \mathbb{Z}^{2},|z| \leq m} U_{z} n_{x, \uparrow} n_{x+z, \downarrow}
$$

o parâmetro $\epsilon \geq 0$ quantifica a energia cinética de férmions ${ }^{1}$. Aqui,

$$
U_{z}: \mathbb{Z}^{2} \rightarrow \mathbb{R}^{+}
$$

é uma função invariante por reflexão que descreve a repulsão entre férmions e $m \in \mathbb{R}^{+}$ representa o alcance da repulsão, no caso de repulsão local $m=0$. Notamos que $n_{x, \downarrow}$, $n_{x+z, \uparrow}$ são os operadores número de partículas para férmions de spin down e spin up

\footnotetext{
${ }^{1} \mathrm{~A}$ amplitude de hopping $\left(-\frac{\epsilon}{2}\right)$ e o potencial químico $(2 \epsilon)$ são escolhidos para ter a mínima energia de referencia no Hamiltoniano como zero, na ausência de repulsão coulombiana. Em geral para $U_{z}=0 \forall z \in \mathbb{Z}^{2}$, temos o Hamiltoniano de Hubbard sem repulsão com parâmetros de hopping $(t)$ e potencial químico $(\mu)$ dado por
}

$$
H=-t \sum_{s \in\{\uparrow, \downarrow\}, x, y \in \mathbb{Z}^{2},|x-y|=1} a_{x, s}^{\dagger} a_{y, s}+\mu \sum_{s \in\{\uparrow, \downarrow\}, x \in \mathbb{Z}^{2}} a_{x, s}^{\dagger} a_{x, s} .
$$

Lembrando que

$$
a_{x, s}=\frac{1}{\sqrt{|\Lambda|}} \sum_{k} \mathrm{e}^{i k \cdot x} a_{k, s}
$$

onde $|\Lambda|$ e ó volume de um setor restringido da rede, escrevemos o Hamiltoniano no espaço de momento.

Tomando no conta que

$$
\begin{aligned}
\sum_{s \in\{\uparrow, \downarrow\}, x, y \in \mathbb{Z}^{2},|x-y|=1} a_{x, s}^{\dagger} a_{y, s}=\frac{1}{|\Lambda|} \sum_{k, k^{\prime}, x, a,|a|=1} \mathrm{e}^{-i k \cdot x} \mathrm{e}^{i k^{\prime} \cdot(x+a)} a_{k, s}^{\dagger} a_{k^{\prime}, s} & \\
& =\sum_{k, s} 2\left(\cos \left(k_{x}\right)+\cos \left(k_{y}\right)\right) a_{k, s}^{\dagger} a_{k, s}
\end{aligned}
$$

e similarmente

$$
\sum_{s \in\{\uparrow, \downarrow\}, x \in \mathbb{Z}^{2}} a_{x, s}^{\dagger} a_{x, s}=\sum_{k, s} a_{k, s}^{\dagger} a_{k, s}=n_{k, s}
$$

temos que

$$
H=\sum_{k, s}(\underbrace{-2 t\left(\cos \left(k_{x}\right)+\cos \left(k_{y}\right)\right)+\mu}_{\varepsilon_{k}}) n_{k, s}
$$

assim o mínimo valor de energia $\varepsilon_{k}$ corresponde a

$$
\varepsilon_{\min }=4 t+\mu
$$

daqui se $\varepsilon_{\min }=0$ temos que $\mu=-4 t$. 
respetivamente, na posição $x \in \mathbb{Z}^{2}$ da rede.

Similarmente o Hamiltoniano do setor bosônico é

$$
H_{b} \doteq \epsilon\left(-\frac{h_{b}}{2} \sum_{x, y \in \mathbb{Z}^{2},|x-y|=1} b_{x}^{\dagger} b_{y}+2 h_{b} \sum_{x \in \mathbb{Z}^{2}} b_{x}^{\dagger} b_{x}\right)
$$

$h_{b} \geq 0$ quantifica a energia dos bósons relativa a dos férmions.

O termo de interação que representa o bipolaron decaindo em dois elétrons, que por sua vez podem formar um bipolaron, é definido como

$$
W^{(\kappa)} \doteq \sum_{x, y \in \mathbb{Z}^{2}} v(x-y)\left(b_{x}^{\dagger} c_{y}^{(\kappa)}+c_{y}^{(\kappa) \dagger} b_{x}\right)
$$

A figura abaixo ilustra os termos da interação elétron-bipolaron.
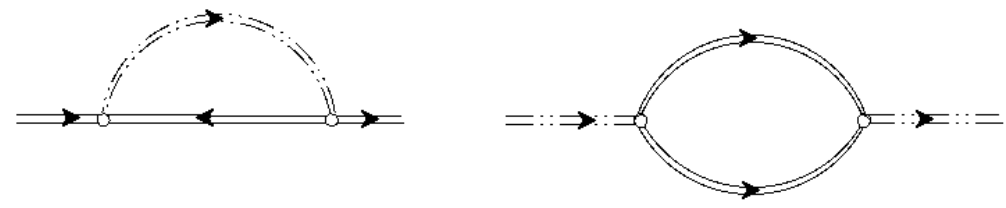

Figura 2.1: A figura da esquerda mostra o decaimento de um par de elétrons em um bipolaron, a da direita o decaimento de um bipolaron em dois elétrons.

$\operatorname{Em}(2.3)$

$$
c_{x}^{(\kappa)} \doteq \sum_{z \in \mathbb{Z}^{2},|z| \leq \gamma \kappa^{-1}} \mathrm{e}^{-\kappa|z|} a_{x+z, \uparrow} a_{x, \downarrow}
$$

onde $c_{x}^{(\kappa)}$ é o operador de destruição de um par eletrônico, e $x \in \mathbb{Z}^{2}$. O parâmetro $\gamma \in \mathbb{R}^{+}$ é fixo (tipicamente $\gamma \sim 1$ ) e $\gamma \kappa^{-1} \in \mathbb{R}^{+}$quantifica o tamanho do bipolaron.

A função de acoplamento $v(x-y)$ é suposta invariante por reflexão e simétrica sob rotações de $90^{\circ}$ tal como se menciono antes. Pode ver-se facilmente que a transformada de Fourier $\hat{v}$ conservará estas simetrias. Como foi dito nas suposições originais, que se sustentam nas observações experimentais, a função $\hat{v}$ deve estar concentrada o ter picos nos pontos $( \pm \pi, 0),(0, \pm \pi)$. 
O Hamiltoniano total será

$$
H \doteq H_{f}^{(m)}+H_{b}+W^{(\kappa)}
$$

Para o caso das três partículas, $H$ atuará sobre o setor correspondente do espaço $\mathcal{F}_{+} \otimes \mathcal{F}_{-}$que deixa invariante o número de partículas, neste caso um bóson e dois férmions. Este espaço será

$$
\mathcal{H}_{\uparrow, \downarrow}^{(2,1)} \doteq\left(\mathfrak{h}_{-,(0)}^{2} \otimes \mathfrak{h}_{+}^{0}\right) \oplus\left(\mathfrak{h}_{-}^{0} \otimes \mathfrak{h}_{+}^{1}\right) \cong \mathfrak{h}_{-,(0)}^{2} \oplus \mathfrak{h}_{+}^{1}
$$

onde $\mathfrak{h}_{-,(0)}^{2}$ denota o espaço de os dois elétrons com spin total zero.

$$
\mathfrak{h}_{-,(0)}^{2} \cong \ell^{2}\left(\mathbb{Z}^{2} ; \mathbb{C}\right) \otimes \ell^{2}\left(\mathbb{Z}^{2} ; \mathbb{C}\right) \cong \ell^{2}\left(\mathbb{Z}^{2} \times \mathbb{Z}^{2} ; \mathbb{C}\right)
$$

Portanto $^{2}$

$$
\mathcal{H}_{\uparrow, \downarrow}^{(2,1)} \cong \ell^{2}\left(\mathbb{Z}^{2} \times \mathbb{Z}^{2} ; \mathbb{C}\right) \times \ell^{2}\left(\mathbb{Z}^{2} ; \mathbb{C}\right)
$$

\footnotetext{
${ }^{2} \mathrm{O}$ isomorfismo em (2.6) resulta natural, seja $\varpi\left(x, x^{\prime}\right) \in \ell^{2}\left(\mathbb{Z}^{2} \times \mathbb{Z}^{2} ; \mathbb{C}\right)$ com $x, x^{\prime} \in \mathbb{Z}^{2}$, então

$$
\varpi\left(x, x^{\prime}\right)=\sum_{a, b} \alpha_{a, b} \delta_{a, b}^{x, x^{\prime}}
$$
}

com

$$
\delta_{a, b}^{x, x^{\prime}}=\left\{\begin{array}{ccc}
1 & \text { se } & \left(x, x^{\prime}\right)=(a, b) \\
0 & \text { se } & \left(x, x^{\prime}\right) \neq(a, b) .
\end{array}\right.
$$

Por outo lado uma base para o espaço $\ell^{2}\left(\mathbb{Z}^{2} ; \mathbb{C}\right) \otimes \ell^{2}\left(\mathbb{Z}^{2} ; \mathbb{C}\right)$ é

$$
\delta_{a}^{x} \otimes \delta_{b}^{x^{\prime}}
$$

$\mathrm{e}$

$$
\delta_{a, b}^{x, x^{\prime}} \cong \delta_{a}^{x} \otimes \delta_{b}^{x^{\prime}}
$$

portanto os espaços $\ell^{2}\left(\mathbb{Z}^{2} ; \mathbb{C}\right) \otimes \ell^{2}\left(\mathbb{Z}^{2} ; \mathbb{C}\right)$ e $\ell^{2}\left(\mathbb{Z}^{2} \times \mathbb{Z}^{2} ; \mathbb{C}\right)$ tem bases ortonormais da mesma cardinalidade. 


\section{Espaço de Dois Férmions e um Bóson}

Como vimos o espaço invariante, para o Hamiltoniano apresentado acima, de dois elétrons e um bipolaron com spin total zero é

$$
\mathcal{H}_{\uparrow, \downarrow}^{(2,1)} \cong \ell^{2}\left(\mathbb{Z}^{2} \times \mathbb{Z}^{2} ; \mathbb{C}\right) \times \ell^{2}\left(\mathbb{Z}^{2} ; \mathbb{C}\right)
$$

Notando que

$$
\ell^{2}\left(\mathbb{Z}^{2} ; \mathbb{C}\right) \cong L^{2}(\underbrace{[-\pi, \pi)^{2}}_{\mathbb{T}^{2}}, d^{2} K ; \mathbb{C})
$$

onde $L^{2}\left(\mathbb{T}^{2}, d^{2} K ; \mathbb{C}\right)$ são as funções do quadrado integrável que vão do torus-2 aos complexos,
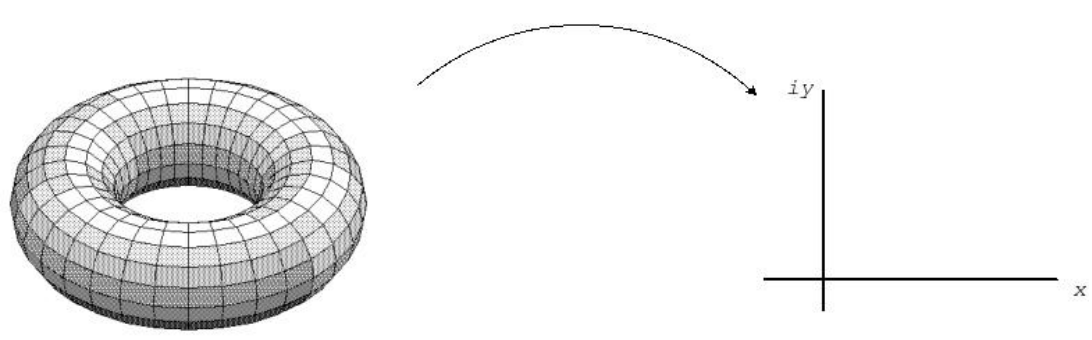

temos que

$$
\ell^{2}\left(\mathbb{Z}^{2} \times \mathbb{Z}^{2} ; \mathbb{C}\right) \cong L^{2}\left(\mathbb{T}^{2} ; L^{2}\left(\mathbb{T}^{2} ; \mathbb{C}\right)\right)=\int_{\mathbb{T}^{2}}^{\oplus} L^{2}\left(\mathbb{T}^{2} ; \mathbb{C}\right) d^{2} K
$$

pelo que

$$
\mathcal{H}_{\uparrow, \downarrow}^{(2,1)} \cong \mathfrak{F}_{\uparrow, \downarrow}^{(2,1)}=\left(\int_{\mathbb{T}^{2}}^{\oplus} L^{2}\left(\mathbb{T}^{2} ; \mathbb{C}\right) d^{2} K\right) \times \underbrace{L^{2}\left(\mathbb{T}^{2} ; \mathbb{C}\right)}_{\int_{\mathbb{T}^{2}}^{\oplus} \mathbb{C} d^{2} K}
$$

Também pode ver-se que

$$
L^{2}\left(X, d \mu ; \mathcal{H}^{\prime}\right) \times L^{2}(X, d \mu ; \mathcal{H}) \cong L^{2}\left(X, d \mu ; \mathcal{H} \times \mathcal{H}^{\prime}\right)
$$

assim

$$
\mathfrak{F}_{\uparrow, \downarrow}^{(2,1)} \cong L^{2}\left(\mathbb{T}^{2} ; L^{2}\left(\mathbb{T}^{2} ; \mathbb{C}\right) \times \mathbb{C}\right)=\int_{\mathbb{T}^{2}}^{\oplus} L^{2}\left(\mathbb{T}^{2} ; \mathbb{C}\right) \times \mathbb{C} d^{2} K
$$


ou seja todo o espaço de os dois elétrons e do bipolaron é isomórfico a integral direta do espaço fibra $L^{2}\left(\mathbb{T}^{2} ; \mathbb{C}\right) \times \mathbb{C}$.

Observamos que

$$
H^{(2,1)} \doteq \overline{\left.H\right|_{\mathcal{H}_{\uparrow, \downarrow}^{(2,1)}}}
$$

é um operador limitado e autoadjunto. Como veremos $H^{(2,1)}$ tem uma imagem unitariamente equivalente $A$, pertencente a $B\left(\mathfrak{F}_{\uparrow, \downarrow}^{(2,1)}\right)$, que permite decomposição em fibra, ou seja,

$$
A \in B_{d}\left(\mathfrak{F}_{\uparrow, \downarrow}^{(2,1)}\right)
$$

e portanto tem associado uma imagem $A(\cdot)$ pertencente ao espaço

$$
L^{\infty}\left(\mathbb{T}^{2} ; B\left(L^{2}\left(\mathbb{T}^{2} ; \mathbb{C}\right) \times \mathbb{C}\right)\right)
$$

\section{Decomposição em Fibras do Hamiltoniano Unitariamente Equivalente a $H^{(2,1)}$}

Seja $\left(\Psi_{\uparrow \downarrow}, \Psi_{b}\right) \in L^{2}\left(\mathbb{T}^{2} ; L^{2}\left(\mathbb{T}^{2} ; \mathbb{C}\right) \times \mathbb{C}\right)$, vamos a definir a transformação unitária

$$
\mathfrak{U}: L^{2}\left(\mathbb{T}^{2} ; L^{2}\left(\mathbb{T}^{2} ; \mathbb{C}\right) \times \mathbb{C}\right) \rightarrow \ell^{2}\left(\mathbb{Z}^{2} \times \mathbb{Z}^{2} ; \mathbb{C}\right) \times \ell^{2}\left(\mathbb{Z}^{2} ; \mathbb{C}\right)
$$

e sua inversa $\mathfrak{U}^{\dagger}$. Temos para o operador $A$ unitariamente equivalente a $H^{(2,1)}$

$$
\begin{aligned}
A\left(\Psi_{\uparrow \downarrow}, \Psi_{b}\right) & =\mathfrak{U}^{\dagger} H^{(2,1)} \mathfrak{U}\left(\Psi_{\uparrow \downarrow}, \Psi_{b}\right) \\
A & \doteq \mathfrak{U}^{\dagger} H^{(2,1)} \mathfrak{U}
\end{aligned}
$$

vemos que,

$$
\begin{gathered}
\mathfrak{U}\left(\Psi_{\uparrow \downarrow}, \Psi_{b}\right) \in \ell^{2}\left(\mathbb{Z}^{2} \times \mathbb{Z}^{2} ; \mathbb{C}\right) \times \ell^{2}\left(\mathbb{Z}^{2} ; \mathbb{C}\right) \\
\mathfrak{U}\left(\Psi_{\uparrow \downarrow}, \Psi_{b}\right)=\left(\phi_{f}\left(x_{\uparrow}, x_{\downarrow}\right), \phi_{b}\left(x_{b}\right)\right)
\end{gathered}
$$


com $x_{\uparrow}, x_{\downarrow}$ e $x_{b} \in \mathbb{Z}^{2}$. As transformações $\mathfrak{U}=\left(\mathfrak{U}_{f}, \mathfrak{U}_{b}\right)$ e $\mathfrak{U}^{\dagger}=\left(\mathfrak{U}_{f}^{\dagger}, \mathfrak{U}_{b}^{\dagger}\right)$ são definidas assim

$$
\mathfrak{U}_{f}\left[\Psi_{\uparrow \downarrow}(K)\right]\left(k_{\uparrow \downarrow}\right)=\phi_{f}\left(x_{\uparrow}, x_{\downarrow}\right) \doteq \frac{1}{(2 \pi)^{4}} \int_{\mathbb{T}^{2}} d^{2} K \int_{\mathbb{T}^{2}} d^{2} k_{\uparrow \downarrow} e^{i K \cdot x_{\uparrow}} e^{i k_{\uparrow \downarrow} \cdot\left(x_{\downarrow}-x_{\uparrow}\right)}\left[\Psi_{\uparrow \downarrow}(K)\right]\left(k_{\uparrow \downarrow}\right)
$$

$\operatorname{com} K, k_{\uparrow \downarrow} \in \mathbb{T}^{2}$. Para $\mathfrak{U}_{b}$ temos

$$
\mathfrak{U}_{b} \phi_{b}\left(x_{b}\right) \doteq \frac{1}{(2 \pi)^{2}} \int_{\mathbb{T}^{2}} e^{i K \cdot x_{b}} \Psi_{b}(K) d^{2} K
$$

Para a transformação $\mathfrak{U}^{\dagger}$ temos

$$
\mathfrak{U}_{f}^{\dagger} \phi_{f}\left(x_{\uparrow}, x_{\downarrow}\right)=\left[\Psi_{\uparrow \downarrow}(K)\right]\left(k_{\uparrow \downarrow}\right) \doteq \sum_{x_{\uparrow}, x_{\uparrow \downarrow} \in \mathbb{Z}^{2}} \mathrm{e}^{-i K \cdot x_{\uparrow}} \mathrm{e}^{-i k_{\uparrow \downarrow} \cdot x_{\uparrow \downarrow}} \phi_{f}\left(x_{\uparrow}, x_{\uparrow}+x_{\uparrow \downarrow}\right)
$$

e

$$
\mathfrak{U}_{b}^{\dagger} \phi_{b}\left(x_{b}\right)=\Psi_{b}(K) \doteq \sum_{x_{b} \in \mathbb{Z}^{2}} \mathrm{e}^{-i K \cdot x_{b}} \phi_{b}\left(x_{b}\right)
$$

Agora calculamos $\mathfrak{U}^{\dagger} H^{(2,1)} \mathfrak{U}$ com o fim de encontrar a forma explicita de $A$,

$$
H^{(2,1)} \mathfrak{U}\left(\Psi_{\uparrow \downarrow}, \Psi_{b}\right)=\left(\phi_{f}^{\prime}, \phi_{b}^{\prime}\right)
$$

O isomorfismo entre o espaço $\mathfrak{h}_{-,(0)}^{2}$ dos dois elétrons e o espaço $\ell^{2}\left(\mathbb{Z}^{2} \times \mathbb{Z}^{2} ; \mathbb{C}\right)$ é escolhido de forma tal que

$$
a_{x, \uparrow} a_{y, \downarrow}\left(\phi_{f}\right)=\phi_{f}(x, y)
$$

o que é natural se vemos a ação do operador $a_{x, \uparrow} a_{y, \downarrow}$ sobre um ket da base original,

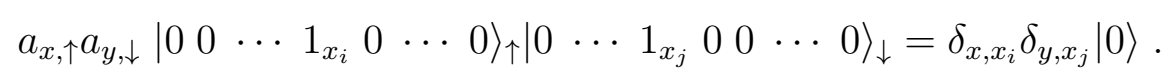

Começando com a parte cinética do Hamiltoniano da parte fermiônica (2.1),

$$
\sum_{s \in\{\uparrow, \downarrow\}, x, y \in \mathbb{Z}^{2},|x-y|=1} a_{x, s}^{\dagger} a_{y, s}\left(\phi_{f}\left(x_{\uparrow}, x_{\downarrow}\right), \phi_{b}\left(x_{b}\right)\right)
$$


ao somar sobre cada spin

$$
\left(\sum_{x, y \in \mathbb{Z}^{2},|x-y|=1} a_{x, \uparrow}^{\dagger} a_{y, \uparrow}+\sum_{x, y \in \mathbb{Z}^{2},|x-y|=1} a_{x, \downarrow}^{\dagger} a_{y, \downarrow}\right)\left(\phi_{f}\left(x_{\uparrow}, x_{\downarrow}\right), \phi_{b}\left(x_{b}\right)\right)
$$

que também pode ser escrito assim

$$
\left(\sum_{x, z \in \mathbb{Z}^{2},|z|=1} a_{x+z, \uparrow}^{\dagger} a_{x, \uparrow}+\sum_{x, z \in \mathbb{Z}^{2},|z|=1} a_{x+z, \downarrow}^{\dagger} a_{x, \downarrow}\right)\left(\phi_{f}\left(x_{\uparrow}, x_{\downarrow}\right), \phi_{b}\left(x_{b}\right)\right) .
$$

O operador $a_{x+z, \uparrow}^{\dagger} a_{x, \uparrow}$ destrói um elétron $\uparrow$ em $x$ e cria um elétron $\uparrow$ em $x+z$, quando a soma em $x$ destes operadores atua sobre a função $\phi_{f}\left(x_{\uparrow}, x_{\downarrow}\right)$, que representa por sua vez uma soma de estados na representação de posição, gera uma traslação

$$
\sum_{x \in \mathbb{Z}^{2}} a_{x+z, \uparrow}^{\dagger} a_{x, \uparrow} \phi_{f}\left(x_{\uparrow}, x_{\downarrow}\right)=\phi_{f}\left(x_{\uparrow}+z, x_{\downarrow}\right)
$$

assim o termo cinético atuando sobre a função $\phi_{f}$ resulta

$$
\sum_{z \in \mathbb{Z}^{2},|z|=1}\left(\phi_{f}\left(x_{\uparrow}+z, x_{\downarrow}\right)+\phi_{f}\left(x_{\uparrow}, x_{\downarrow}+z\right)\right)
$$

O seguinte termo no Hamiltoniano (2.1), chamado do potencial químico, deixa invariante a função

$$
\sum_{x \in \mathbb{Z}^{2}} a_{x, \uparrow}^{\dagger} a_{x, \uparrow} \phi_{f}\left(x_{\uparrow}, x_{\downarrow}\right)=\phi_{f}\left(x_{\uparrow}, x_{\downarrow}\right)
$$

portanto

$$
\sum_{s \in\{\uparrow, \downarrow\}, x \in \mathbb{Z}^{2}} a_{x, s}^{\dagger} a_{x, s} \phi_{f}\left(x_{\uparrow}, x_{\downarrow}\right)=2 \phi_{f}\left(x_{\uparrow}, x_{\downarrow}\right) .
$$

Para a repulsão coulombiana, o último termo do Hamiltoniano (2.1), temos

$$
\sum_{x \in \mathbb{Z}^{2},|z| \leq m} U_{z} n_{x, \uparrow} n_{x+z, \downarrow} \phi_{f}\left(x_{\uparrow}, x_{\downarrow}\right)
$$

Observemos a ação do operador sobre um elemento da base original do espaço de os 
dois elétrons

$$
\sum_{x^{\prime} \in \mathbb{Z}^{2},|z| \leq m} U_{z} n_{x^{\prime}, \uparrow} n_{x^{\prime}+z, \downarrow} \mid \begin{array}{lllllllllllll}
0 & 0 & \cdots & 1_{x_{i}} & 0 & \cdots & 0\rangle_{\uparrow} \mid 0 & \cdots & 1_{x_{j}} & 0 & 0 & \cdots & 0\rangle_{\downarrow}
\end{array}
$$

então podemos escrever o termo anterior assim

$$
\sum_{x^{\prime} \in \mathbb{Z}^{2},|z| \leq m} U_{z} \delta_{x^{\prime}+z, x_{j}} \delta_{x^{\prime}, x_{i}} \mid \begin{array}{lllllllllllll}
0 & 0 & \cdots & 1_{x_{i}} & 0 & \cdots & 0\rangle_{\uparrow} \mid 0 & \cdots & 1_{x_{j}} & 0 & 0 & \cdots & 0\rangle_{\downarrow}
\end{array}
$$

o que é igual a

$$
\sum_{|z| \leq m} U_{z} \delta_{x_{i}+z, x_{j}}\left|\begin{array}{lllllllllllll}
0 & 0 & \cdots & 1_{x_{i}} & 0 & \cdots & 0\rangle_{\uparrow} \mid 0 & \cdots & 1_{x_{j}} & 0 & 0 & \cdots & 0
\end{array}\right\rangle_{\downarrow}
$$

Então temos

$$
\sum_{x^{\prime} \in \mathbb{Z}^{2},|z| \leq m} U_{z} n_{x^{\prime}, \uparrow} n_{x^{\prime}+z, \downarrow} \phi_{f}\left(x_{\uparrow}, x_{\downarrow}\right)=\sum_{|z| \leq m} U_{z} \delta_{x_{\uparrow}+z, x_{\downarrow}} \phi_{f}\left(x_{\uparrow}, x_{\downarrow}\right) .
$$

Finalmente calculamos a ação do operador $W^{(\kappa)}(2.3)$ sobre $\left(\phi_{f}\left(x_{\uparrow}, x_{\downarrow}\right), \phi_{b}\left(x_{b}\right)\right)$,

$$
\sum_{x, y \in \mathbb{Z}^{2}} v(x-y)\left(b_{x}^{\dagger} c_{y}^{(\kappa)}+c_{y}^{(\kappa) \dagger} b_{x}\right)\left(\phi_{f}\left(x_{\uparrow}, x_{\downarrow}\right), \phi_{b}\left(x_{b}\right)\right)
$$

primeiro observamos o efeito de $c_{y}^{(\kappa)}$,

$$
c_{y}^{(\kappa)}\left(\phi_{f}\left(x_{\uparrow}, x_{\downarrow}\right), \phi_{b}\left(x_{b}\right)\right)=\sum_{z \in \mathbb{Z}^{2},|z| \leq \gamma \kappa^{-1}} \mathrm{e}^{-\kappa|z|} a_{y+z, \uparrow} a_{y, \downarrow}\left(\phi_{f}\left(x_{\uparrow}, x_{\downarrow}\right), \phi_{b}\left(x_{b}\right)\right)
$$

como vimos antes

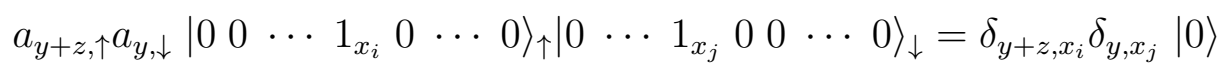


portanto

$$
\begin{aligned}
& \sum_{x, y \in \mathbb{Z}^{2}} v(x-y) b_{x}^{\dagger} c_{y}^{(\kappa)} \phi_{f}\left(x_{\uparrow}, x_{\downarrow}\right) \otimes \phi_{b, 0} \\
&=\sum_{x, y \in \mathbb{Z}^{2}} v(x-y) b_{x}^{\dagger} \sum_{z \in \mathbb{Z}^{2},|z| \leq \gamma \kappa^{-1}} \mathrm{e}^{-\kappa|z|} \delta_{y+z, x_{\uparrow}} \delta_{y, x_{\downarrow}} \phi_{f, 0} \otimes \phi_{b, 0} \\
&=\sum_{x_{b}} \sum_{|z| \leq \gamma \kappa^{-1}} e^{-\kappa|z|} v\left(x_{b}-x_{\downarrow}\right) \delta_{x_{\uparrow}+z, x_{\downarrow}} \phi_{b}\left(x_{b}\right)
\end{aligned}
$$

onde $\phi_{f, 0}$ e $\phi_{b, 0}$ denotam o vácuo fermiônico e bosônico respectivamente. Finalmente para $\phi_{f}^{\prime}$ temos

$$
\begin{aligned}
\phi_{f}^{\prime}\left(x_{\uparrow}, x_{\downarrow}\right)= & -\frac{\epsilon}{2} \sum_{z \in \mathbb{Z}^{2},|z|=1} \phi_{f}\left(x_{\uparrow}+z, x_{\downarrow}\right)-\frac{\epsilon}{2} \sum_{z \in \mathbb{Z}^{2},|z|=1} \phi_{f}\left(x_{\uparrow}, x_{\downarrow}+z\right) \\
& +4 \epsilon \phi_{f}\left(x_{\uparrow}, x_{\downarrow}\right)+\sum_{|z| \leq m} U_{z} \delta_{x_{\uparrow}, x_{\downarrow}+z} \phi_{f}\left(x_{\uparrow}, x_{\downarrow}\right) \\
& +\sum_{x_{b}} \sum_{|z| \leq \gamma n} \mathrm{e}^{-\kappa|z|} v\left(x_{b}-x_{\downarrow}\right) \delta_{x_{\uparrow}+z, x_{\downarrow}} \phi_{b}\left(x_{b}\right) .
\end{aligned}
$$

Similarmente, para o termino de interação atuando sobre a parte bosônica temos

$$
\begin{aligned}
\sum_{x, y \in \mathbb{Z}^{2}} v(x-y) c_{y}^{(\kappa) \dagger} b_{x} \phi_{f, 0} \otimes \phi_{b}\left(x_{b}\right) & =\sum_{y \in \mathbb{Z}^{2}} v\left(x_{b}-y\right) \sum_{z \in \mathbb{Z}^{2},|z| \leq \gamma \kappa^{-1}} \mathrm{e}^{-\kappa|z|} a_{y, \downarrow}^{\dagger} a_{y+z, \uparrow}^{\dagger} \phi_{f, 0} \otimes \phi_{b, 0} \\
& =\sum_{x \in \mathbb{Z}^{2}} \sum_{z \in \mathbb{Z}^{2},|z| \leq \gamma \kappa^{-1}} \mathrm{e}^{-\kappa|z|} v\left(x_{b}-x\right) \phi_{f}(x+z, x)
\end{aligned}
$$

assim temos que

$\phi_{b}^{\prime}\left(x_{b}\right)=-\frac{\epsilon h_{b}}{2} \sum_{z \in \mathbb{Z}^{2},|z|=1} \phi_{b}\left(x_{b}+z\right)+2 \epsilon h_{b} \phi_{b}\left(x_{b}\right)+\sum_{x \in \mathbb{Z}^{2}} \sum_{z \in \mathbb{Z}^{2},|z| \leq \gamma \kappa^{-1}} \mathrm{e}^{-\kappa|z|} v\left(x_{b}-x\right) \phi_{f}(x+z, x)$.

Agora para encontrar a forma do Hamiltoniano unitariamente equivalente no espaço $B\left(\mathfrak{F}_{\uparrow, \downarrow}^{(2,1)}\right)$ aplicamos a transformação inversa $\mathfrak{U}^{\dagger}$, para a função fermiônica

$$
\left[\Psi_{\uparrow \downarrow}^{\prime}(K)\right]\left(k_{\uparrow \downarrow}\right)=\sum_{x_{\uparrow}, x_{\uparrow \downarrow} \in \mathbb{Z}^{2}} \mathrm{e}^{-i K \cdot x_{\uparrow}} e^{-i k_{\uparrow \downarrow} \cdot x_{\uparrow \downarrow}} \phi_{f}^{\prime}\left(x_{\uparrow}, x_{\uparrow}+x_{\uparrow \downarrow}\right)
$$


para o primeiro término de $\phi_{f}^{\prime}$ correspondente a parte cinética temos

$$
-\frac{\epsilon}{2} \sum_{x_{\uparrow}, x_{\uparrow \downarrow} \in \mathbb{Z}^{2}, z \in \mathbb{Z}^{2},|z|=1} \mathrm{e}^{-i K \cdot x_{\uparrow}} \mathrm{e}^{-i k_{\uparrow \downarrow} \cdot x_{\uparrow \downarrow}}\left(\phi_{f}\left(x_{\uparrow}+z, x_{\downarrow}\right)+\phi_{f}\left(x_{\uparrow}, x_{\downarrow}+z\right)\right) .
$$

Assim

$\sum_{x_{\uparrow}, x_{\uparrow \downarrow} \in \mathbb{Z}^{2}, z \in \mathbb{Z}^{2},|z|=1} \mathrm{e}^{-i K \cdot x_{\uparrow}} \mathrm{e}^{-i k_{\uparrow \downarrow} \cdot x_{\uparrow \downarrow}} \phi_{f}\left(x_{\uparrow}+z, x_{\downarrow}\right)=\sum_{z \in \mathbb{Z}^{2},|z|=1} \mathrm{e}^{i\left(K-k_{\uparrow \downarrow}\right) \cdot z}\left[\Psi_{\uparrow \downarrow}(K)\right]\left(k_{\uparrow \downarrow}\right)$

$$
=2 \cos \left(K-k_{\uparrow \downarrow}\right)\left[\Psi_{\uparrow \downarrow}(K)\right]\left(k_{\uparrow \downarrow}\right)
$$

onde $\cos \left(K-k_{\uparrow \downarrow}\right) \doteq \cos \left(K_{x}-k_{\uparrow \downarrow, x}\right)+\cos \left(K_{y}-k_{\uparrow \downarrow}, y\right)$. Similarmente

$$
\sum_{x_{\uparrow}, x_{\uparrow \downarrow} \in \mathbb{Z}^{2}, z \in \mathbb{Z}^{2},|z|=1} \mathrm{e}^{-i K \cdot x_{\uparrow}} \mathrm{e}^{-i k_{\uparrow \downarrow} \cdot x_{\uparrow \downarrow}} \phi_{f}\left(x_{\uparrow}, x_{\downarrow}+z\right)=2 \cos \left(k_{\uparrow \downarrow}\right)\left[\Psi_{\uparrow \downarrow}(K)\right]\left(k_{\uparrow \downarrow}\right) .
$$

Para inverter o seguinte termo, $4 \epsilon \phi_{f}\left(x_{\uparrow}, x_{\downarrow}\right)$, na equação (2.12) fica direto. Calculamos agora a inversa para a repulsão coulombiana

$$
\sum_{x_{\uparrow}, x_{\uparrow \downarrow} \in \mathbb{Z}^{2}} \mathrm{e}^{-i K \cdot x_{\uparrow}} \mathrm{e}^{-i k_{\uparrow \downarrow} \cdot x_{\uparrow \downarrow}} \sum_{|z| \leq m} U_{z} \delta_{x_{\uparrow}, x_{\downarrow}+z} \phi_{f}\left(x_{\uparrow}, x_{\downarrow}\right)
$$

Reescrevendo $\phi_{f}\left(x_{\uparrow}, x_{\downarrow}\right)$ temos

$$
\sum_{x_{\uparrow}, x_{\uparrow \downarrow} \in \mathbb{Z}^{2}} \mathrm{e}^{-i K \cdot x_{\uparrow}} \mathrm{e}^{-i k_{\uparrow \downarrow} \cdot x_{\uparrow \downarrow}} \sum_{|z| \leq m} U_{z} \delta_{x_{\uparrow}, x_{\downarrow}+z} \frac{1}{(2 \pi)^{4}} \int_{\mathbb{T}^{2}} d^{2} K \int_{\mathbb{T}^{2}} d^{2} k_{\uparrow \downarrow} \mathrm{e}^{i K \cdot x_{\uparrow}} \mathrm{e}^{i k_{\uparrow \downarrow} \cdot\left(x_{\downarrow}-x_{\uparrow}\right)}\left[\Psi_{\uparrow \downarrow}(K)\right]\left(k_{\uparrow \downarrow}\right) .
$$

Somando em $x_{\uparrow \downarrow}$

$$
\sum_{x_{\uparrow},|z| \leq m} \mathrm{e}^{-i K \cdot x_{\uparrow}} \mathrm{e}^{-i k_{\uparrow \downarrow} \cdot z} U_{z} \frac{1}{(2 \pi)^{4}} \int_{\mathbb{T}^{2}} d^{2} K \int_{\mathbb{T}^{2}} d^{2} k_{\uparrow \downarrow} \mathrm{e}^{i K \cdot x_{\uparrow}} \mathrm{e}^{i k_{\uparrow \downarrow} \cdot z}\left[\Psi_{\uparrow \downarrow}(K)\right]\left(k_{\uparrow \downarrow}\right)
$$


Notamos que

$$
\begin{array}{r}
\sum_{|z| \leq m} \mathrm{e}^{-i k_{\uparrow \downarrow} \cdot z} U_{z} \sum_{x_{\uparrow}} \mathrm{e}^{-i K \cdot x_{\uparrow}} \frac{1}{(2 \pi)^{2}} \int_{\mathbb{T}^{2}} d^{2} K \mathrm{e}^{i K \cdot x_{\uparrow}} \underbrace{\frac{1}{(2 \pi)^{2}} \int_{\mathbb{T}^{2}} d^{2} k_{\uparrow \downarrow} \mathrm{e}^{i k_{\uparrow \downarrow} \cdot z}\left[\Psi_{\uparrow \downarrow}(K)\right]\left(k_{\uparrow \downarrow}\right)}_{\varphi(K, z)} \\
=\sum_{|z| \leq m} \mathrm{e}^{-i k_{\uparrow \downarrow} \cdot z} U_{z} \varphi(K, z)
\end{array}
$$

que pode ser escrito assim

$$
\sum_{|z| \leq m} U_{z}\left|\mathrm{e}^{-i k_{\uparrow \downarrow} \cdot z}\right\rangle\left\langle\mathrm{e}^{-i k_{\uparrow \downarrow} \cdot z},\left[\Psi_{\uparrow \downarrow}(K)\right]\left(k_{\uparrow \downarrow}\right)\right\rangle=\sum_{|z| \leq m} U_{z} P_{z}\left[\Psi_{\uparrow \downarrow}(K)\right]\left(k_{\uparrow \downarrow}\right)
$$

com $P_{z}=\left|\mathrm{e}^{-i k_{\uparrow \downarrow} \cdot z}\right\rangle\left\langle\mathrm{e}^{-i k_{\uparrow \downarrow} \cdot z}\right|$, sendo $\langle\cdot, \cdot\rangle$ o produto interno de $L^{2}\left(\mathbb{T}^{2} ; \mathbb{C}\right)$

$$
\langle\phi, \Psi\rangle:=\frac{1}{(2 \pi)^{2}} \int_{\mathbb{T}^{2}} d^{2} k^{\prime} \phi^{*}\left(k^{\prime}\right) \Psi\left(k^{\prime}\right)
$$

Para o último termo na equação (2.12) temos

$$
\sum_{x_{\uparrow}, x_{\uparrow \downarrow} \in \mathbb{Z}^{2}} \mathrm{e}^{-i K \cdot x_{\uparrow}} \mathrm{e}^{-i k_{\uparrow \downarrow} \cdot x_{\uparrow \downarrow}} \sum_{x_{b}} \sum_{|z| \leq \gamma \kappa^{-1}} \mathrm{e}^{-\kappa|z|} v\left(x_{b}-x_{\downarrow}\right) \delta_{x_{\uparrow}+z, x_{\downarrow}} \phi_{b}\left(x_{b}\right) .
$$

Reordenando a soma

$$
\sum_{x_{\uparrow}, x_{\downarrow} \in \mathbb{Z}^{2}} \mathrm{e}^{-i\left(K-k_{\uparrow \downarrow}\right) \cdot x_{\uparrow}} \mathrm{e}^{-i k_{\uparrow \downarrow} \cdot x_{\downarrow}} \sum_{x_{b}} \sum_{|z| \leq \gamma \kappa^{-1}} \mathrm{e}^{-\kappa|z|} v\left(x_{b}-x_{\downarrow}\right) \delta_{x_{\uparrow}+z, x_{\downarrow}} \phi_{b}\left(x_{b}\right)
$$

somando em $x_{\uparrow}$

$$
\sum_{x_{\downarrow}, z \in \mathbb{Z}^{2}|| z \mid \leq \gamma \kappa^{-1}} \mathrm{e}^{-\kappa|z|} \mathrm{e}^{-i\left(K-k_{\uparrow \downarrow}\right) \cdot\left(x_{\downarrow}-z\right)} \mathrm{e}^{-i k_{\uparrow \downarrow} \cdot x_{\downarrow}} \sum_{x_{b}} v\left(x_{b}-x_{\downarrow}\right) \phi_{b}\left(x_{b}\right)
$$

que é igual a

$$
\sum_{|z| \leq \gamma \kappa^{-1}} \mathrm{e}^{-\kappa|z|} \mathrm{e}^{i\left(K-k_{\uparrow \downarrow}\right) \cdot z} \sum_{x_{\downarrow} \in \mathbb{Z}^{2}} \mathrm{e}^{-i K \cdot x_{\downarrow}} \sum_{x_{b}} v\left(x_{b}-x_{\downarrow}\right) \phi_{b}\left(x_{b}\right)
$$


pelo teorema da convolução

$$
\sum_{x_{\downarrow} \in \mathbb{Z}^{2}} \mathrm{e}^{-i K \cdot x_{\downarrow}} \sum_{x_{b}} v\left(x_{b}-x_{\downarrow}\right) \phi_{b}\left(x_{b}\right)=\hat{v}(K) \Psi_{b}(K)
$$

com

$$
\hat{v}(K)=\sum_{x \in \mathbb{Z}^{2}} \mathrm{e}^{-i K \cdot x} v(x)
$$

observando também que

$$
\sum_{|z| \leq \gamma \kappa^{-1}} \mathrm{e}^{-\kappa|z|} \mathrm{e}^{i\left(K-k_{\uparrow \downarrow}\right) \cdot z}=1+\left[f^{(\gamma, \kappa)}(K)\right]\left(k_{\uparrow \downarrow}\right)
$$

com

$$
\left[f^{(\gamma, \kappa)}(K)\right]\left(k_{\uparrow \downarrow}\right)=2 \sum_{0<n^{\prime} \leq \gamma \kappa^{-1}} \mathrm{e}^{-\kappa n^{\prime}} \cos \left(n^{\prime}\left(k_{\uparrow \downarrow}-K\right)\right)+4 \sum_{n^{\prime}, m^{\prime}>0} \sum_{\left|z^{\prime}\right|<\gamma \kappa^{-1}} e^{-\kappa\left|z^{\prime}\right|}\left[g_{n^{\prime}, m^{\prime}}(K)\right]\left(k_{\uparrow \downarrow}\right)
$$

onde

$$
\left[g_{n^{\prime}, m^{\prime}}(K)\right]\left(k_{\uparrow \downarrow}\right)=\cos \left(n^{\prime}\left(k_{\uparrow \downarrow x}-K_{x}\right)\right) \cos \left(m^{\prime}\left(k_{\uparrow \downarrow y}-K_{y}\right)\right)
$$

temos que (2.14) pode escrever-se assim

$$
\sum_{|z| \leq \gamma \kappa^{-1}} \mathrm{e}^{-\kappa|z|} \mathrm{e}^{i\left(K-k_{\uparrow \downarrow}\right) \cdot z} \sum_{x_{\downarrow} \in \mathbb{Z}^{2}} \mathrm{e}^{-i K \cdot x_{\downarrow}} \sum_{x_{b}} v\left(x_{b}-x_{\downarrow}\right) \phi_{b}\left(x_{b}\right)=\hat{v}(K)\left(1+\left[f^{(\gamma, \kappa)}(K)\right]\left(k_{\uparrow \downarrow}\right)\right) \Psi_{b}(K) .
$$

Para a função bosônica

$$
\Psi_{b}^{\prime}(K)=\sum_{x_{b} \in \mathbb{Z}^{2}} \mathrm{e}^{-i K \cdot x_{b}} \phi_{b}^{\prime}\left(x_{b}\right)
$$

temos analogamente para a parte cinética

$$
\sum_{x_{b} \in \mathbb{Z}^{2}} \mathrm{e}^{-i K \cdot x_{b}} \sum_{z \in \mathbb{Z}^{2}} \phi_{b}\left(x_{b}+z\right)=2 \cos (K) \Psi_{b}(K)
$$


e para o termo de potencial químico diretamente

$$
\sum_{x_{b} \in \mathbb{Z}^{2}} \mathrm{e}^{-i K \cdot x_{b}} \phi_{b}\left(x_{b}\right)=\Psi_{b}(K)
$$

Para a parte de interação bipolaron-elétron no (2.13) temos

$$
\sum_{x_{b} \in \mathbb{Z}^{2}} \mathrm{e}^{-i K \cdot x_{b}} \sum_{x \in \mathbb{Z}^{2}} \sum_{z \in \mathbb{Z}^{2},|z| \leq \gamma \kappa^{-1}} \mathrm{e}^{-\kappa|z|} v\left(x_{b}-x\right) \phi_{f}(x+z, x)
$$

reordenando e usando novamente o teorema da convolução podemos escrever a expressão anterior assim

$$
\hat{v}(K) \sum_{z \in \mathbb{Z}^{2},|z| \leq \gamma \kappa^{-1}} \mathrm{e}^{-\kappa|z|} \mathfrak{U}_{b}^{\dagger} \phi_{f}(x+z, x) .
$$

Agora

$\mathfrak{U}_{b}^{\dagger} \phi_{f}(x+z, x)=\sum_{x \in \mathbb{Z}^{2}} \mathrm{e}^{-i K \cdot x} \frac{1}{(2 \pi)^{2}} \int_{\mathbb{T}^{2}} d^{2} K \mathrm{e}^{i K \cdot x} \frac{1}{\left(2 \pi^{2}\right)} \int_{\mathbb{T}^{2}} d^{2} k_{\uparrow \downarrow} \mathrm{e}^{i\left(K-k_{\uparrow \downarrow}\right) \cdot z}\left[\Psi_{\uparrow \downarrow}(K)\right]\left(k_{\uparrow \downarrow}\right)$

pelo que (2.15) pode escrever-se assim

$$
\begin{array}{r}
\hat{v}(K) \sum_{x \in \mathbb{Z}^{2}} \mathrm{e}^{-i K \cdot x} \frac{1}{(2 \pi)^{2}} \int_{\mathbb{T}^{2}} d^{2} K \mathrm{e}^{i K \cdot x} \underbrace{\frac{1}{(2 \pi)^{2}} \int_{\mathbb{T}^{2}} d^{2} k_{\uparrow \downarrow} \sum_{z \in \mathbb{Z}^{2},|z| \leq \gamma \kappa^{-1}} \mathrm{e}^{-\kappa|z|} \mathrm{e}^{i\left(K-k_{\uparrow \downarrow}\right) \cdot z}\left[\Psi_{\uparrow \downarrow}(K)\right]\left(k_{\uparrow \downarrow}\right)}_{\psi(K)} \\
=\hat{v}(K) \psi(K) \quad(2.16)
\end{array}
$$

$\mathrm{Ou}$

$$
\hat{v}(K) \psi(K)=\hat{v}(K)\left\langle 1+\left[f^{(\gamma, \kappa)}(K)\right]\left(k_{\uparrow \downarrow}\right),\left[\Psi_{\uparrow \downarrow}(K)\right]\left(k_{\uparrow \downarrow}\right)\right\rangle .
$$


Finalmente com todos os cálculos obtemos

$$
\begin{aligned}
{\left[\Psi_{\uparrow \downarrow}^{\prime}(K)\right]\left(k_{\uparrow \downarrow}\right)=\epsilon(4-} & \left.\cos \left(k_{\uparrow \downarrow}-K\right)-\cos \left(k_{\uparrow \downarrow}\right)\right)\left[\Psi_{\uparrow \downarrow}(K)\right]\left(k_{\uparrow \downarrow}\right) \\
& +\sum_{|z| \leq m} U_{z} \mathrm{e}^{-i k_{\uparrow \downarrow} \cdot z}\left\langle\mathrm{e}^{-i k_{\uparrow \downarrow} \cdot z},\left[\Psi_{\uparrow \downarrow}(K)\right]\left(k_{\uparrow \downarrow}\right)\right\rangle \\
& +\hat{v}(K)\left(1+\left[f^{(n)}(K)\right]\left(k_{\uparrow \downarrow}\right)\right) \Psi_{b}(K)
\end{aligned}
$$

e

$$
\Psi_{b}^{\prime}(K)=\epsilon h_{b}(2-\cos (K)) \Psi_{b}(K)+\hat{v}(K)\left\langle 1+\left[f^{(n)}(K)\right]\left(k_{\uparrow \downarrow}\right),\left[\Psi_{\uparrow \downarrow}(K)\right]\left(k_{\uparrow \downarrow}\right)\right\rangle
$$

o que também pode escrever-se no forma matricial

$$
\left(\begin{array}{c}
{\left[\Psi_{\uparrow \downarrow}^{\prime}(K)\right]\left(k_{\uparrow \downarrow}\right)} \\
\Psi_{b}^{\prime}(K)
\end{array}\right)=\left(\begin{array}{cc}
A_{1,1}(K) & A_{1,2}(K) \\
A_{2,1}(K) & A_{2,2}(K)
\end{array}\right)\left(\begin{array}{c}
{\left[\Psi_{\uparrow \downarrow}(K)\right]\left(k_{\uparrow \downarrow}\right)} \\
\Psi_{b}(K)
\end{array}\right)=A(K)\left(\begin{array}{c}
{\left[\Psi_{\uparrow \downarrow}(K)\right]\left(k_{\uparrow \downarrow}\right)} \\
\Psi_{b}(K)
\end{array}\right)
$$

com

$$
\begin{aligned}
& A_{1,1}(K) \doteq \epsilon\left(4-\cos \left(k_{\uparrow \downarrow}-K\right)-\cos \left(k_{\uparrow \downarrow}\right)\right)+\sum_{|z| \leq m} U_{z} P_{z} \\
& A_{1,2}(K) \doteq \hat{v}(K)\left(1+\left[f^{(n)}(K)\right]\left(k_{\uparrow \downarrow}\right)\right) \\
& A_{2,1}(K) \doteq \hat{v}(K)\left\langle 1+\left[f^{(n)}(K)\right]\left(k_{\uparrow \downarrow}\right), \cdot\right\rangle \\
& A_{2,2}(K) \doteq \epsilon h_{b}(2-\cos (K))
\end{aligned}
$$


onde os operadores limitados estão definidos nos espaços

$$
\begin{aligned}
& A_{1,1}(K): L^{2}\left(\mathbb{T}^{2} ; \mathbb{C}\right) \rightarrow L^{2}\left(\mathbb{T}^{2} ; \mathbb{C}\right) \\
& A_{1,2}(K): \mathbb{C} \rightarrow L^{2}\left(\mathbb{T}^{2} ; \mathbb{C}\right) \\
& A_{2,1}(K): L^{2}\left(\mathbb{T}^{2} ; \mathbb{C}\right) \rightarrow \mathbb{C} \\
& A_{2,2}(K): \mathbb{C} \rightarrow \mathbb{C} .
\end{aligned}
$$

Observamos que $A(\cdot)$ é essencialmente limitado, portanto pertence ao espaço $L^{\infty}\left(\mathbb{T}^{2} ; B\left(L^{2}\left(\mathbb{T}^{2} ; \mathbb{C}\right) \times \mathbb{C}\right)\right)$. Temos assim que $A$, o operador unitariamente equivalente a $H^{(2,1)}$, pode ser decomposto no fibras $A(K)$

$$
A=\int_{\mathbb{T}^{2}}^{\oplus} A(K) d^{2} K
$$

\section{Espectro de $A$ e a Fibra $A(K)$}

Vimos que no espaço $\mathfrak{F}_{\uparrow, \downarrow}^{(2,1)}$ é possível descrever a função de onda dos dois elétrons e o bipolaron em termos de os dois parâmetros $K$ e $k_{\uparrow \downarrow}$, e que o Hamiltoniano $A$ pertencente ao espaço $B\left(\mathfrak{F}_{\uparrow, \downarrow}^{(2,1)}\right)$ pode ser decomposto em fibras, o que simplificará o análise do problema. Na relação (1.4) pode observar-se a o vinculo entre o espectro do operador $A$ e o espectro de suas fibras $A(x)$.

Seja $E_{0}=\min \sigma(A)$, então $P_{A}\left(\left(E_{0}-\delta\right)-\epsilon,\left(E_{0}-\delta\right)+\epsilon\right)=0$ para algum $\delta>0$, onde $P_{A}(\lambda)$ é a projeção espetral associada a $A$ como vimos antes. A relação (1.4) e consequência de

$$
P_{A}(\lambda-\epsilon, \lambda+\epsilon)=\int_{X}^{\oplus} P_{A(x)}(\lambda-\epsilon, \lambda+\epsilon) d \mu
$$

pelo que podemos observar que se $P_{A}\left(\left(E_{0}-\delta\right)-\epsilon,\left(E_{0}-\delta\right)+\epsilon\right)=0$, isto implica que $P_{A(x)}\left(\left(E_{0}-\delta\right)-\epsilon,\left(E_{0}-\delta\right)+\epsilon\right)=0$ a.e. . 
No nosso caso $A(K)$ é contínuo e portanto ess $\sup _{K \in \mathbb{T}^{2}}\|A(K)\|=\sup _{K \in \mathbb{T}^{2}}\|A(K)\|$, daí junto com a teoria de Kato para perturbação do espectro discreto de operadores fechados, temos que

$$
P_{A}\left(\left(E_{0}-\delta\right)-\epsilon,\left(E_{0}-\delta\right)+\epsilon\right)=0 \Rightarrow P_{A(K)}\left(\left(E_{0}-\delta\right)-\epsilon,\left(E_{0}-\delta\right)+\epsilon\right)=0 \forall K \in \mathbb{T}^{2}
$$

$\mathrm{e}$

$$
E_{0}=\min \sigma(A)=\min \sigma\left(H^{(2,1)}\right)=\min _{K \in \mathbb{T}^{2}}\{\min (\sigma(A(K)))\}
$$

Do anterior vemos que podemos encontrar a energia fundamental $E_{0}$ de $A$ a partir do mínimo valor do espectro das fibras $A(K)$.

Pode ser observado que se o mínimo valor do espectro de $A(K)$ é menor que zero, tem que ser um autovalor, isto do fato que $A_{1,2}(K), A_{2,1}(K)$ e $\sum_{|z| \leq m} U_{z} P_{z}$ são operadores limitados de range finito dimensional e portanto compactos. Como

$$
\sigma(A)=\sigma_{e s s}(A) \cup \sigma_{d}(A)
$$

$\mathrm{e}$

$$
\sigma_{e s s}(A)=\sigma_{e s s}(A+\tilde{K})
$$

onde $\tilde{K}$ e um operador compacto, se tem que

$$
\sigma_{e s s}(A)=\sigma(\widetilde{A})
$$

onde

$$
\widetilde{A}=\left(\begin{array}{cc}
\epsilon\left(4-\cos \left(k_{\uparrow \downarrow}-K\right)-\cos \left(k_{\uparrow \downarrow}\right)\right) & 0 \\
0 & \epsilon h_{b}(2-\cos (K))
\end{array}\right)
$$

Como $\widetilde{A}$ é um operador multiplicativo seu espectro será igual a seu rango essencial, portanto

$$
\sigma_{e s s}(A) \subset[0,8 \epsilon]
$$




\section{Autovalores Negativos das Fibras $A(K)$}

Como vimos na seção anterior se existe um valor negativo do espectro de a fibra $A(K)$ este deve ser um autovalor. Calcularemos agora os possíveis autovalores negativos $\lambda$ para $A(K)$, temos então as equações de autovalores

$$
\left(\begin{array}{c}
\left(A_{1,1}(K)-\lambda\right) \Psi_{\uparrow \downarrow}+A_{1,2}(K) \Psi_{b} \\
A_{2,1}(K) \Psi_{\uparrow \downarrow}+\left(A_{2,2}(K)-\lambda\right) \Psi_{b}
\end{array}\right)=\left(\begin{array}{l}
0 \\
\\
0
\end{array}\right)
$$

com $\Psi_{\uparrow \downarrow} \neq 0$ e $\Psi_{b} \neq 0$. Uma vez que $U_{z}, \epsilon, h_{b}>0$, se $\lambda<0$ temos que $\left(A_{1,1}(K)-\lambda\right)$ e $\left(A_{2,2}(K)-\lambda\right)$ são estritamente positivos e portanto invertíveis.

Para o cálculo seguinte se usará uma versão do principio de Birman-Schwinger, cujo enunciado é

Seja $d \geq 1$ e $H_{0}, V \in \mathcal{B}\left(\ell^{2}\left(\mathbb{Z}^{d}\right)\right)$ operadores positivos e limitados. Suponhamos que $V$ é compacto. Para qualquer $\lambda<0$, definimos o compacto, autoadjunto e positivo operador de Birman-Schwinger

$$
B(\lambda)=B\left(\lambda, H_{0}, V\right):=V^{1 / 2}\left(H_{0}-\lambda\right)^{-1} V^{1 / 2}
$$

então $\lambda<0$ é um autovalor de $\left(H_{0}-V\right)$ de multiplicidade $M$ se e somente se 1 é um autovalor de $B(\lambda)$ de multiplicidade $M$.

Do par de equações de autovalores temos

$$
\left[\left(A_{1,1}(K)-\lambda\right)-A_{1,2}(K)\left(A_{2,2}(K)-\lambda\right)^{-1} A_{2,1}(K)\right] \Psi_{\uparrow \downarrow}=0
$$

observamos que para um $K$ fixo, $\left(A_{2,2}(K)-\lambda\right)^{-1}=\left(\epsilon h_{b}(2-\cos (K))-\lambda\right)^{-1}$ é constante, e $A_{1,2}(K)$ ficara atuando sobre $A_{2,1}(K)$, agora vemos que

$$
A_{1,2}(K) A_{2,1}(K)=|\hat{v}(K)|^{2}\|\mathfrak{f}\|^{2} P_{\mathfrak{f}}
$$


com

$$
\mathfrak{f} \doteq 1+\left[f^{(\gamma, \kappa)}(K)\right]\left(k_{\uparrow \downarrow}\right)
$$

e

$$
P_{\mathfrak{f}} \doteq \frac{1}{\|\mathfrak{f}\|^{2}}|\mathfrak{f}\rangle\langle\mathfrak{f}|
$$

De a equação (2.21) vemos que

$$
\left(A_{1,1}(K)-A_{1,2}(K)\left(A_{2,2}(K)-\lambda\right)^{-1} A_{2,1}(K)\right) \Psi_{\uparrow \downarrow}=\lambda \Psi_{\uparrow \downarrow}
$$

portanto resulta natural usar o principio de Birman-Schwinger com $H_{0}=A_{1,1}(K)$ and $V=A_{1,2}(K)\left(A_{2,2}(K)-\lambda\right)^{-1} A_{2,1}(K)$, como

$$
V^{1 / 2}=\left(\epsilon h_{b}(2-\cos (K))-\lambda\right)^{-1 / 2}|\hat{v}(K)|\|\mathfrak{f}\| P_{\mathfrak{f}}
$$

temos

$$
\left(\epsilon h_{b}(2-\cos (K))-\lambda\right)^{-1}|\hat{v}(K)|^{2}\|\mathfrak{f}\|^{2} P_{\mathfrak{f}}\left(A_{1,1}(K)-\lambda\right)^{-1} P_{\mathfrak{f}} \varphi=\varphi
$$

para algum $\varphi \in L^{2}\left(\mathbb{T}^{2} ; \mathbb{C}\right)$. Aplicando o operador $\langle\mathfrak{f}, \cdot\rangle$ na equação anterior vemos que

$$
|\hat{v}(K)|^{2} \mathcal{R}(K, \lambda)+\lambda-\epsilon h_{b}(2-\cos (K))=0
$$

onde $\mathcal{R}(K, \lambda)$ é definido assim

$$
\mathcal{R}(K, \lambda) \doteq \mathfrak{R}_{\mathfrak{f}, \mathfrak{f}}=\left\langle\mathfrak{f},\left(A_{1,1}(K)-\lambda\right)^{-1} \mathfrak{f}\right\rangle
$$

A equação (2.22) permite calcular os possíveis autovalores negativos da fibra $A(K)$, agora é preciso ter uma forma explicita para o elemento de matriz $\mathfrak{R}_{\mathfrak{f}, \mathfrak{f}}$.

O elemento $A_{1,1}(K)$ pode escrever-se assim

$$
A_{1,1}(K)=A_{1,1}^{(0)}(K)+\sum_{|z| \leq m} U_{z} P_{z}
$$


onde $A_{1,1}^{(0)}(K)=\epsilon\left(4-\cos \left(k_{\uparrow \downarrow}-K\right)-\cos \left(k_{\uparrow \downarrow}\right)\right)$. Da equação resolvente

$$
R(\lambda)=R^{(0)}(\lambda)-R^{(0)}(\lambda)\left(\sum_{|z| \leq m} U_{z} P_{z}\right) R(\lambda)
$$

onde

$$
R(\lambda)=\left(A_{1,1}(K)-\lambda\right)^{-1}
$$

e

$$
R^{(0)}(\lambda)=\left(A_{1,1}^{(0)}(K)-\lambda\right)^{-1}
$$

nós temos que

$$
\mathfrak{R}_{j, k}=\mathfrak{R}_{j, k}^{(0)}-\sum_{i|| i \mid \leq m} U_{i} \mathfrak{R}_{j, i}^{(0)} \mathfrak{R}_{i, k}
$$

com $j, k \in I^{(m)}$, onde $m \geq 0$,

$$
I^{(m)}:=\left\{z \in \mathbb{Z}^{2}|| z \mid \leq m\right\} \cup\{\mathfrak{f}\}
$$

e os elementos das matrizes, de ordem $n\left(I^{(m)}\right) \times n\left(I^{(m)}\right), \mathfrak{R}$ e $\mathfrak{R}^{(0)}$ são definidos assim

$$
\begin{gathered}
\mathfrak{R}_{l, m}:=\left\langle e^{i k_{\uparrow \downarrow} \cdot l},\left(A_{1,1}(K)-\lambda\right)^{-1} e^{-i k_{\uparrow \uparrow} \cdot m}\right\rangle \\
\mathfrak{R}_{\mathrm{l}, \mathfrak{f}}:=\left\langle e^{i k_{\uparrow \downarrow} \cdot l},\left(A_{1,1}(K)-\lambda\right)^{-1} \mathfrak{f}\right\rangle
\end{gathered}
$$

com $l, m \in I^{(m)} \cap \mathbb{Z}^{2}$, e como foi definido em (2.23)

$$
\mathfrak{R}_{\mathfrak{f}, \mathfrak{f}}:=\left\langle\mathfrak{f},\left(A_{1,1}(K)-\lambda\right)^{-1} \mathfrak{f}\right\rangle
$$

Assim mesmo definimos os elementos de $\mathfrak{R}^{(0)}$

$$
\begin{gathered}
\mathfrak{R}_{l, m}^{(0)}:=\left\langle e^{i k_{\uparrow \downarrow} \cdot l},\left(A_{1,1}^{(0)}(K)-\lambda\right)^{-1} e^{-i k_{\uparrow \downarrow} \cdot m}\right\rangle \\
\mathfrak{R}_{\mathfrak{l}, \mathfrak{f}}^{(0)}:=\left\langle\mathrm{e}^{i k_{\uparrow \downarrow} \cdot l},\left(A_{1,1}^{(0)}(K)-\lambda\right)^{-1} \mathfrak{f}\right\rangle
\end{gathered}
$$


$\operatorname{com} l, m \in I^{(m)} \cap \mathbb{Z}^{2}, \mathrm{e}$

$$
\mathfrak{R}_{\mathfrak{f}, \mathfrak{f}}^{(0)}:=\left\langle\mathfrak{f},\left(A_{1,1}^{(0)}(K)-\lambda\right)^{-1} \mathfrak{f}\right\rangle
$$

Outra matriz de ordem $n\left(I^{(m)}\right) \times n\left(I^{(m)}\right)$ útil a definir é $\tilde{\mathfrak{R}}^{(0)}$

$$
\begin{gathered}
\tilde{\mathfrak{R}}_{k, i}^{(0)}:=U_{i} \mathfrak{R}_{k, i}^{(0)} . \\
\mathfrak{R}_{i, \mathfrak{f}}:=0
\end{gathered}
$$

$\operatorname{com} i \in I^{(m)}$ e $k \in I^{(m)} \cap \mathbb{Z}^{2}$.

Das equações (2.24) e (2.25), temos

$$
\mathfrak{R}=\mathfrak{R}^{(0)}-\tilde{\mathfrak{R}}^{(0)} \mathfrak{R}
$$

ou equivalentemente

$$
\mathfrak{R}=\left(\tilde{\mathfrak{R}}^{(0)}+\mathbb{1}\right)^{-1} \mathfrak{R}^{(0)}
$$

Da equação matricial (2.27) encontramos o elemento $\mathfrak{R}_{\mathfrak{f}, \mathfrak{f}}$.

O calculo de o elemento $\mathfrak{R}_{\mathfrak{f}, \mathfrak{f}}$ e muito extenso e não é possível fazer analiticamente ao menos que as matrizes não sejam grandes. O caso mais simples onde pode calcular-se $\mathfrak{R}_{\mathfrak{f}, \mathfrak{f}}$ de forma analítica é quando a repulsão é local, sendo o valor do parâmetro $m=0$ no Hamiltoniano (2.1).

Para o caso de repulsão local podemos observar que

$$
\begin{aligned}
\mathfrak{R} & =\left(\begin{array}{ll}
\mathfrak{R}_{0,0} & \mathfrak{R}_{0, \mathfrak{f}} \\
\mathfrak{R}_{\mathfrak{f}, 0} & \mathfrak{R}_{\mathfrak{f}, \mathfrak{f}}
\end{array}\right) \\
\mathfrak{R}^{(0)} & =\left(\begin{array}{ll}
\mathfrak{R}_{0,0}^{(0)} & \mathfrak{R}_{0, \mathfrak{f}}^{(0)} \\
\mathfrak{R}_{\mathfrak{f}, 0}^{(0)} & \mathfrak{R}_{\mathfrak{f}, \mathfrak{f}}^{(0)}
\end{array}\right)
\end{aligned}
$$




$$
\left(\tilde{\mathfrak{R}}^{(0)}+\mathbb{1}\right)^{-1}=\frac{1}{1+U_{0} \mathfrak{R}_{0,0}^{(0)}}\left(\begin{array}{cc}
1 & 0 \\
-U_{0} \mathfrak{R}_{0, \mathfrak{f}}^{(0)} & U_{0} \mathfrak{R}_{0,0}+1
\end{array}\right)
$$

portanto

$$
\mathfrak{R}=\left(\begin{array}{cc}
\mathfrak{R}_{0,0} & \mathfrak{R}_{0, \mathfrak{f}} \\
\mathfrak{R}_{\mathfrak{f}, 0} & \mathfrak{R}_{\mathfrak{f}, \mathfrak{f}}
\end{array}\right)=\frac{1}{1+U_{0} \mathfrak{R}_{0,0}^{(0)}}\left(\begin{array}{cc}
1 & 0 \\
-U_{0} \mathfrak{R}_{0, \mathfrak{f}}^{(0)} & U_{0} \mathfrak{R}_{0,0}+1
\end{array}\right)\left(\begin{array}{cc}
\mathfrak{R}_{0,0}^{(0)} & \mathfrak{R}_{0, \mathfrak{f}}^{(0)} \\
\mathfrak{R}_{\mathfrak{f}, 0}^{(0)} & \mathfrak{R}_{\mathfrak{f}, \mathfrak{f}}^{(0)}
\end{array}\right)
$$

e

$$
\mathfrak{R}_{\mathfrak{f}, \mathfrak{f}}=\frac{\mathfrak{R}_{\mathfrak{f}, \mathfrak{f}}^{(0)}}{1+U_{0} \mathfrak{R}_{0,0}^{(0)}}+U_{0} \frac{\mathfrak{R}_{\mathfrak{f}, \mathfrak{f}}^{(0)} \mathfrak{R}_{0,0}^{(0)}-\left(\mathfrak{R}_{0, \mathfrak{f}}^{(0)}\right)^{2}}{1+U_{0} \mathfrak{R}_{0,0}^{(0)}}
$$

onde explicitamente temos

$$
\begin{aligned}
& \mathfrak{R}_{0,0}^{(0)}=\frac{1}{(2 \pi)^{2}} \int_{\mathbb{T}^{2}} \frac{1}{\epsilon\left(4-\cos \left(k_{\uparrow \downarrow}-K\right)-\cos \left(k_{\uparrow \downarrow}\right)\right)-\lambda} d^{2} k_{\uparrow \downarrow} \\
& \mathfrak{R}_{\mathfrak{\uparrow}, \mathrm{f}}^{(0)}=\frac{1}{(2 \pi)^{2}} \int_{\mathbb{T}^{2}} \frac{\left(1+\left[f^{(\gamma, \kappa)}(K)\right]\left(k_{\uparrow \downarrow}\right)\right)^{2}}{\epsilon\left(4-\cos \left(k_{\uparrow \downarrow}-K\right)-\cos \left(k_{\uparrow \downarrow}\right)\right)-\lambda} d^{2} k_{\uparrow \downarrow} \\
& \mathfrak{R}_{0, f}^{(0)}=\frac{1}{(2 \pi)^{2}} \int_{\mathbb{T}^{2}} \frac{1+\left[f^{(\gamma, \kappa)}(K)\right]\left(k_{\uparrow \downarrow}\right)}{\epsilon\left(4-\cos \left(k_{\uparrow \downarrow}-K\right)-\cos \left(k_{\uparrow \downarrow}\right)\right)-\lambda} d^{2} k_{\uparrow \downarrow} .
\end{aligned}
$$

No caso mais simples de interesse $\gamma \kappa^{-1}=1 \mathrm{em}(2.4)$, podemos observar que

$$
1+\left[f^{(\gamma, \kappa)}(K)\right]\left(k_{\uparrow \downarrow}\right)=1+2 \mathrm{e}^{-\kappa} \cos \left(k_{\uparrow \downarrow}-K\right)
$$

A existência e o cálculo de uma solução $\lambda<0$ para a equação (2.22) pode ser explorada com cálculos numéricos. Para o caso $\gamma \kappa^{-1}=1$ e $m=0$ presentamos abaixo a simulação para a função $|\hat{v}(K)|^{2} \mathcal{R}(K, \lambda)$ e a reta $\epsilon h_{b}(2-\cos (K))-\lambda$ mostrando que existe uma solução negativa para a equação (2.22). 


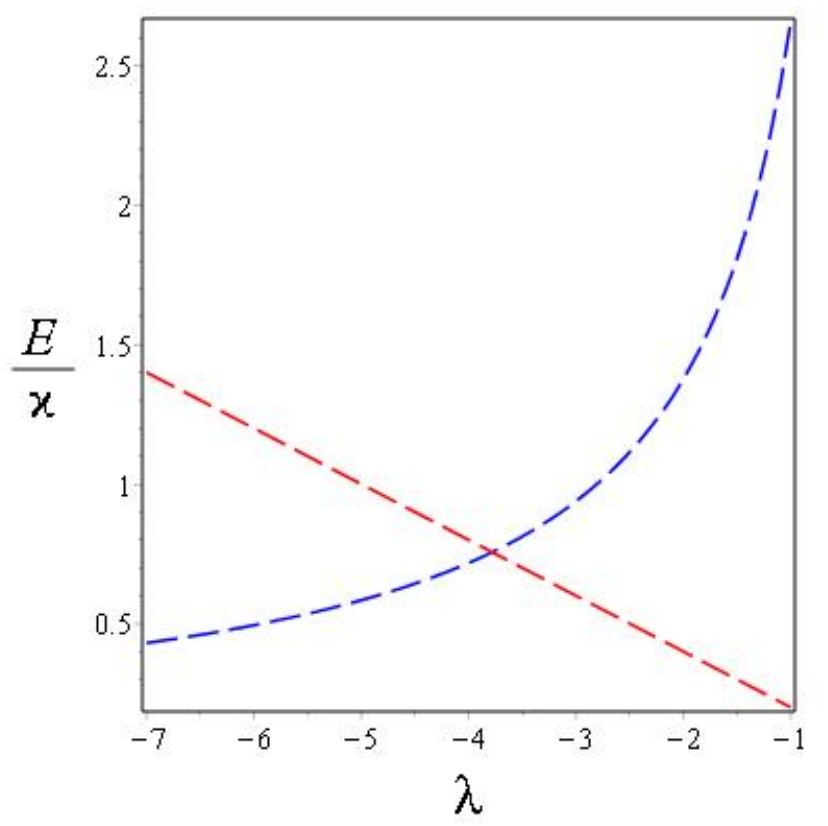

Figura 2.2: No azul esta a função $|\hat{v}(K)|^{2} \mathcal{R}(K, \lambda)$ e no vermelho $\epsilon h_{b}(2-\cos (K))-\lambda$ com os parâmetro $U_{0}=100, K_{x}=0$ e $K_{y}=\pi$.

A energia na figura acima esta nas unidades de $\varkappa \in \mathbb{R}^{+}, \varkappa$ é o parâmetro que quantifica a força da interação bipolaron-elétron, ou seja corresponde ao máximo da função $\varkappa \hat{v}(K)$. Como está nas proposições do modelo, a função $\hat{v}(K)$ deve ter picos nos pontos $(0, \pm \pi)$, $( \pm \pi, 0)$ onde a interação bipolaron-elétron é forte. Presentamos abaixo o gráfico da função $\hat{v}(K)$ escolhida, semelhante a escolhida em $[8,9]$. 


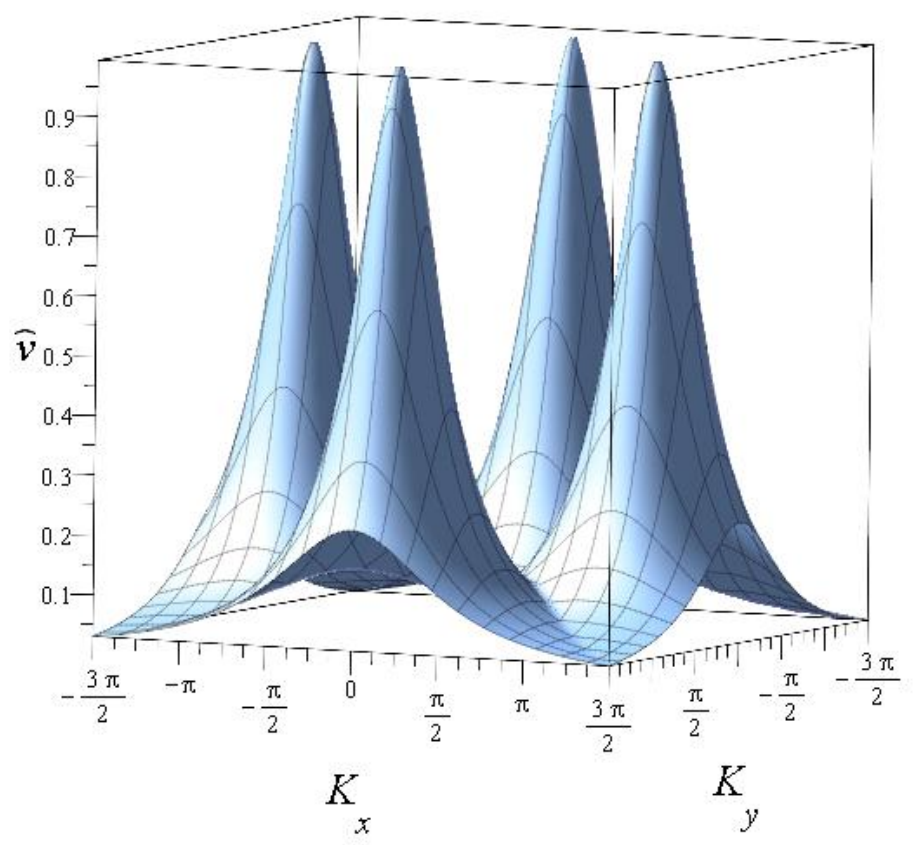

Figura 2.3: Função acoplamento $\hat{v}$.

$$
\begin{array}{r}
\hat{v}\left(K_{x}, K_{y}\right)=\max \left\{\frac{1}{\alpha\left(\left(K_{x}-\pi\right)^{2}+K_{y}^{2}\right)+1}, \frac{1}{\alpha\left(\left(K_{x}+\pi\right)^{2}+K_{y}^{2}\right)+1},\right. \\
\left.\frac{1}{\alpha\left(K_{x}^{2}+\left(K_{y}-\pi\right)^{2}\right)+1}, \frac{1}{\alpha\left(K_{x}^{2}+\left(K_{y}+\pi\right)^{2}\right)+1}\right\} .
\end{array}
$$

Pode ser visto também como varia o autovalor para distintas fibras $A(K)$. Abaixo apresentamos alguns cálculos numéricos. 


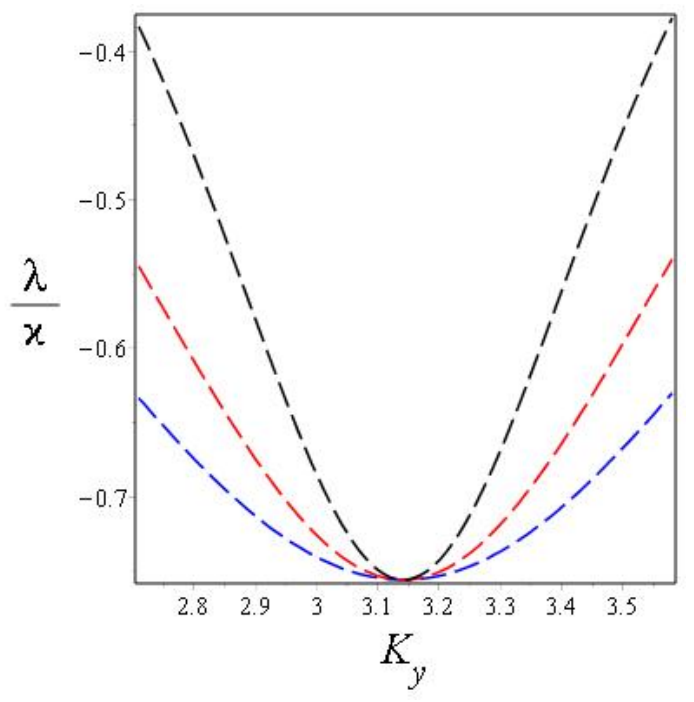

Figura 2.4: Variação do autovalor para distintos valores de $\alpha$. Para a curva azul $\alpha=1$, a vermelha $\alpha=2$ e a preta $\alpha=5$. Os parâmetros escolhidos são $K_{x}=0, \varkappa=5, \epsilon=0,01$, $U_{0}=100$ e $h_{b}=0,2$.

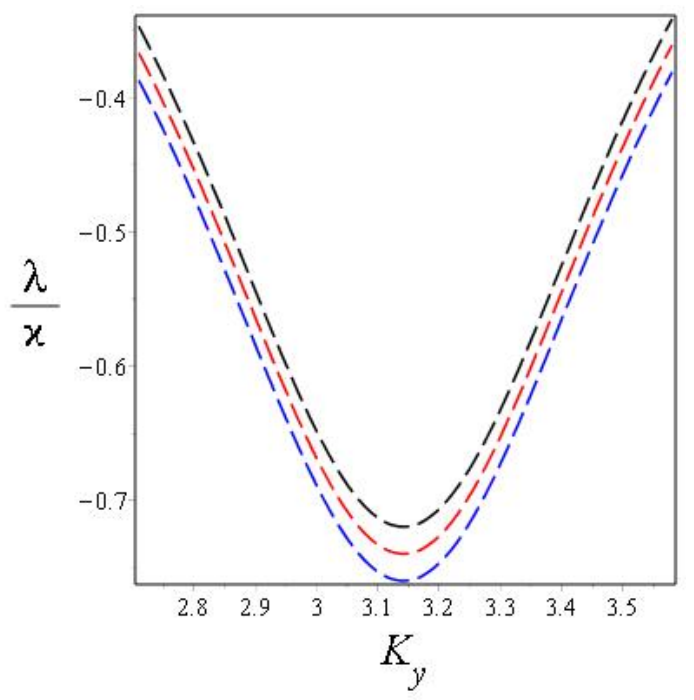

Figura 2.5: Variação do autovalor para distintos valores de a energia $\epsilon$. Para a curva azul $\epsilon=0,001$, a vermelha $\epsilon=0,05$ e a preta $\epsilon=0,1$. Os parâmetros escolhidos são $K_{x}=0$, $\alpha=5, \varkappa=5, U_{0}=100$, e $h_{b}=0,2$. 


\section{Estabilidade da Formação de Pares de Férmions}

Como vimos se existir um eigenvalor para a fibra $A(k)$ este deve ser negativo, e

$$
E_{0}=\min \sigma(A)=\min \sigma\left(H^{(2,1)}\right)=\min _{K \in \mathbb{T}^{2}}\{\min (\sigma(A(K)))\}
$$

Vemos então que o valor mínimo do espectro $E_{0}$ de $H^{(2,1)}$ será negativo se o conjunto de eigenvalores $\left\{\lambda \mid \lambda \in \sigma_{d}(A(k))\right\} \neq\{\emptyset\}$. Seja $\left(\phi_{f}\left(x_{\uparrow}, x_{\downarrow}\right), \phi_{b}\left(x_{b}\right)\right)_{(0)}$ o estado base do $H^{(2,1)}$, então

$$
\left(\phi_{f}\left(x_{\uparrow}, x_{\downarrow}\right), \phi_{b}\left(x_{b}\right)\right)_{(t)}=\mathrm{e}^{-i t E_{0}}\left(\phi_{f}\left(x_{\uparrow}, x_{\downarrow}\right), \phi_{b}\left(x_{b}\right)\right)_{(0)}
$$

Como discutimos anteriormente $E_{0}$ corresponde ao mínimo autovalor das fibras $A(K)$, ou $E_{0}=\min _{K \in \mathbb{T}^{2}} E(K)$, onde

$$
A(K)\left(\left[\Psi_{\uparrow \downarrow}(K)\right]\left(k_{\uparrow \downarrow}\right), \Psi_{b}(K)\right)=E(K)\left(\left[\Psi_{\uparrow \downarrow}(K)\right]\left(k_{\uparrow \downarrow}\right), \Psi_{b}(K)\right)
$$

portanto

$$
\begin{aligned}
\left(\Psi_{\uparrow \downarrow}, \Psi_{b}\right)_{(t)}=\int_{\mathbb{T}^{2}}^{\oplus} \mathrm{e}^{-i t A(K)}\left(\left[\Psi_{\uparrow \downarrow}(K)\right]\left(k_{\uparrow \downarrow}\right), \Psi_{b}(K)\right) d^{2} K \\
=\int_{\mathbb{T}^{2}}^{\oplus} \mathrm{e}^{-i t E(K)}\left(\left[\Psi_{\uparrow \downarrow}(K)\right]\left(k_{\uparrow \downarrow}\right), \Psi_{b}(K)\right) d^{2} K .
\end{aligned}
$$

Se $E_{0}$ existe, então também existe uma autofunção fibra $\left(\left[\Psi_{\uparrow \downarrow}(K)\right]\left(k_{\uparrow \downarrow}\right), \Psi_{b}(K)\right) \neq 0$, portanto

$$
\left\|\Psi_{\uparrow \downarrow}(t)\right\|^{2}>0 \Rightarrow\left\|\phi_{f}(t)\right\|^{2}>0 \forall t
$$

Também observamos do fato

$$
\left\|\phi_{f}(t)\right\|^{2}=\sum_{x_{\uparrow}, x_{\downarrow} \in \mathbb{Z}^{2}}\left|\phi_{f}\left(x_{\uparrow}, x_{\downarrow}\right)_{(t)}\right|^{2}=\left\|\phi_{f}(0)\right\|^{2}
$$

que

$$
\sum_{x_{\uparrow}, x_{\downarrow} \in \mathbb{Z}^{2}|| x_{\uparrow}-x_{\downarrow} \mid<R}\left|\phi_{f}\left(x_{\uparrow}, x_{\downarrow}\right)_{(t)}\right|^{2} \leq\left\|\phi_{f}(0)\right\|^{2}
$$


dando-se a igualdade só no caso $R \rightarrow \infty$. Portanto para todo tempo existirá uma probabilidade diferente de zero de encontrar pares de elétrons.

\section{Existência de Simetria $d$-wave no Parâmetro de Ordem}

Agora calcularemos em detalhe a autofunção fermiônica correspondente ao estado fundamental com o fim de estudar sua simetria. Como é sabido a função que descreve o par de elétrons acoplados no regime supercondutor é o chamado parâmetro de ordem da transição. Heuristicamente temos uma função em termos da coordenada relativa do par de elétrons

$$
\psi\left(r_{\uparrow}-r_{\downarrow}\right)=\sum_{k} g(k) \mathrm{e}^{i k \cdot\left(r_{\uparrow}-r_{\downarrow}\right)}
$$

onde a função $g(k)$ é isotrópica no caso do supercondutores típicos, chamados também BCS. Se diz que tais supercondutores apresentam "supercondutividade s-wave". Como se mencionou anteriormente nos supercondutores de alta $T_{c}$ foi observado a presença de supercondutividade $d$-wave, ou seja a função $g(k)$ não é isotrópica e presenta inversão de sinal sob rotações de $90^{\circ}$ no espaço $k$,

$$
g\left(k_{x}, k_{y}\right)=-g\left(k_{y},-k_{x}\right)
$$

Mostraremos agora que a autofunção do estado base no nosso modelo presenta simetria $d$-wave.

Da primeira equação para a autofunção da fibra $A(K)$

$$
\left(\begin{array}{c}
\left(A_{1,1}(K)-\lambda\right) \Psi_{\uparrow \downarrow}+A_{1,2}(K) \Psi_{b} \\
A_{2,1}(K) \Psi_{\uparrow \downarrow}+\left(A_{2,2}(K)-\lambda\right) \Psi_{b}
\end{array}\right)=\left(\begin{array}{l}
0 \\
\\
0
\end{array}\right)
$$

temos

$$
\Psi_{\uparrow \downarrow}=-\left(A_{1,1}(K)-\lambda\right)^{-1} A_{1,2}(K) \Psi_{b} .
$$


Considerando $\Psi_{b}=1$

$$
\Psi_{\uparrow \downarrow}=-\left(A_{1,1}(K)-\lambda\right)^{-1} \hat{v}(K) \mathfrak{f}
$$

e usando (2.24) temos

$$
\Psi_{\uparrow \downarrow}=-\left(A_{1,1}^{(0)}(K)-\lambda\right)^{-1} \hat{v}(K)\left(\mathfrak{f}-\sum_{|z| \leq m} U_{z} e^{-i k_{\uparrow \downarrow} \cdot z} \mathfrak{R}_{z, \mathfrak{f}}\right) .
$$

Primeiro consideremos o caso de repulsão local e $\gamma \kappa^{-1}=1$, que permite tratamento analítico. O caso não local e com $\gamma \kappa^{-1}>1$ será considerado no capitulo seguente mediante calculações numéricas.

Para $m=0$ e $\gamma \kappa^{-1}=1$ temos

$$
\Psi_{\uparrow \downarrow}=-\left(A_{1,1}^{(0)}(K)-\lambda\right)^{-1} \hat{v}(K)\left(\left(1+2 \mathrm{e}^{-\kappa} \cos \left(k_{\uparrow \downarrow}-K\right)\right)-U_{0} R_{\mathfrak{s , f}}\right)
$$

Da equação (2.27) para o caso $m=0$, temos

$$
\begin{gathered}
\mathfrak{R}_{0, \mathfrak{f}}=\frac{\mathfrak{R}_{0, \mathfrak{f}}^{(0)}}{1+U_{0} \mathfrak{R}_{0,0}^{(0)}} \\
\mathfrak{R}_{0, f}=\frac{\left\langle 1,\left(A_{1,1}^{(0)}(K)-\lambda\right)^{-1}\left(1+2 \mathrm{e}^{-\kappa} \cos \left(k_{\uparrow \downarrow}-K\right)\right)\right\rangle}{1+U_{0}\left\langle 1,\left(A_{1,1}^{(0)}(K)-\lambda\right)^{-1} 1\right\rangle}
\end{gathered}
$$

então temos

$$
\Psi_{\uparrow \downarrow}=-\frac{\hat{v}(K)\left(\left(1+2 \mathrm{e}^{-\kappa} \cos \left(k_{\uparrow \downarrow}-K\right)\right)-\frac{U_{0}\left\langle 1,\left(A_{1,1}^{(0)}(K)-\lambda\right)^{-1}\left(1+2 \mathrm{e}^{-\kappa} \cos \left(k_{\uparrow \downarrow}-K\right)\right)\right\rangle}{1+U_{0}\left\langle 1,\left(A_{1,1}^{(0)}(K)-\lambda\right)^{-1} 1\right\rangle}\right)}{\epsilon\left(4-\cos \left(k_{\uparrow \downarrow}-K\right)-\cos \left(k_{\uparrow \downarrow}\right)\right)-E(K)}
$$

onde $\lambda=E(K)$.

Como pode ser observado nas figuras 2.4 e 2.5, o espectro das fibras é mais localizado a medida que $\alpha$ cresce e a mínima energia $E_{0}$ é mais negativa a medida que $\epsilon$ decresce. Justamente a região de interesse a ser explorada é quando $\epsilon \rightarrow 0$ e $\alpha \rightarrow \infty$. 
No limite $\alpha \rightarrow \infty E(K)$ será muito localizado nos pontos onde $\hat{v}(K)$ tem os picos, então podemos assumir neste limite

$$
E(K)=E_{0} \chi^{\varepsilon}(K)
$$

onde definimos

$$
\chi^{\varepsilon}(K)=\left\{\begin{array}{ccc}
1 & \text { se } & K \in\left[k_{m, x}-\varepsilon, k_{m, x}+\varepsilon\right] \times\left[k_{m, y}-\varepsilon, k_{m, y}+\varepsilon\right] \\
0 & \text { se } \quad K \notin\left[k_{m, x}-\varepsilon, k_{m, x}+\varepsilon\right] \times\left[k_{m, y}-\varepsilon, k_{m, y}+\varepsilon\right]
\end{array}\right.
$$

para $k_{m}=\{(0, \pi),(0,-\pi),(\pi, 0),(-\pi, 0)\}, \varepsilon \gtrsim 0 \in \mathbb{R}$ e $E_{0}<0$ o mínimo do espectro.

Também definimos

$$
\hat{\mu}(K)=\lim _{\alpha \rightarrow \infty} \hat{v}(K) \approx \varkappa \chi^{\varepsilon}(K)
$$

De (2.30) vemos que se $\epsilon \rightarrow 0$ e $\alpha \rightarrow \infty$

$$
\Psi_{\uparrow \downarrow} \rightarrow \chi^{\varepsilon}(K) \frac{\varkappa}{E_{0}}\left(\left(1+2 \mathrm{e}^{-\kappa} \cos \left(k_{\uparrow \downarrow}-K\right)\right)-\frac{U_{0}\left\langle 1, \frac{1}{E_{0}}\left(1+2 \mathrm{e}^{-\kappa} \cos \left(k_{\uparrow \downarrow}-K\right)\right)\right\rangle}{1+U_{0}\left\langle 1, \frac{1}{E_{0}} 1\right\rangle}\right) .
$$

Agora a função no espaço da posição é

$$
\phi_{f}\left(x_{\uparrow}, x_{\downarrow}\right)=\frac{1}{(2 \pi)^{4}} \int_{\mathbb{T}^{2}} d^{2} k_{\uparrow \downarrow} \mathrm{e}^{i k_{\uparrow \downarrow} \cdot x_{\uparrow \downarrow}} \int_{\mathbb{T}^{2}} d^{2} K \mathrm{e}^{i K \cdot x_{\uparrow}}\left[\Psi_{\uparrow \downarrow}(K)\right]\left(k_{\uparrow \downarrow}\right) .
$$

Para $\epsilon \rightarrow 0$ e $\alpha \rightarrow \infty$, a integral $\int_{\mathbb{T}^{2}} d^{2} K \mathrm{e}^{i K \cdot x_{\uparrow}}\left[\Psi_{\uparrow \downarrow}(K)\right]\left(k_{\uparrow \downarrow}\right)$ tende a

$$
\sum_{K \in k_{m}} 4 \varepsilon^{2} \mathrm{e}^{i K \cdot x_{\uparrow}} \frac{\varkappa}{E_{0}}\left(\left(1+2 \mathrm{e}^{-\kappa} \cos \left(k_{\uparrow \downarrow}-K\right)\right)-\frac{\frac{U_{0}}{E_{0}}}{1+\frac{U_{0}}{E_{0}}}\right) \doteq \widetilde{\Psi}_{\uparrow \downarrow}\left(k_{\uparrow \downarrow}\right)
$$

e

$$
\phi_{f}\left(x_{\uparrow}, x_{\downarrow}\right) \rightarrow \frac{1}{(2 \pi)^{4}} \int_{\mathbb{T}^{2}} d^{2} k_{\uparrow \downarrow} e^{i k_{\uparrow \downarrow} \cdot x_{\uparrow \downarrow}} \widetilde{\Psi}_{\uparrow \downarrow}\left(k_{\uparrow \downarrow}\right) .
$$


Agora calculamos $\phi_{f}\left(x_{\uparrow}, x_{\downarrow}\right)$,

$$
\phi_{f}\left(x_{\uparrow}, x_{\downarrow}\right)=\sum_{K \in k_{m}} \frac{4 \varepsilon^{2}}{(2 \pi)^{4}} \mathrm{e}^{i K \cdot x_{\uparrow}} \frac{\varkappa}{E_{0}} \int_{\mathbb{T}^{2}} d^{2} k_{\uparrow \downarrow} \mathrm{e}^{i k_{\uparrow \downarrow} \cdot x_{\uparrow \downarrow}}\left(\frac{1}{1+\frac{U_{0}}{E_{0}}}+2 \mathrm{e}^{-\kappa} \cos \left(k_{\uparrow \downarrow}-K\right)\right)
$$

como

$$
\int_{\mathbb{T}^{2}} d^{2} k_{\uparrow \downarrow} \mathrm{e}^{i k_{\uparrow \downarrow} \cdot x_{\uparrow \downarrow}}=(2 \pi)^{2} \delta_{x_{\uparrow \downarrow}, 0}=(2 \pi)^{2} \delta_{x_{\uparrow}, x_{\downarrow}}
$$

e

$$
\begin{array}{r}
\int_{\mathbb{T}^{2}} d^{2} k_{\uparrow \downarrow} \mathrm{e}^{i k_{\uparrow \downarrow} \cdot x_{\uparrow \downarrow}} \cos \left(k_{\uparrow \downarrow}-K\right)=\int_{\mathbb{T}^{2}} d^{2} k_{\uparrow \downarrow} \mathrm{e}^{i k_{\uparrow \downarrow} \cdot x_{\uparrow \downarrow}}\left(\cos \left(k_{\uparrow \downarrow}, x-K_{x}\right)+\cos \left(k_{\uparrow \downarrow}, y-K_{y}\right)\right) \\
=\frac{(2 \pi)^{2}}{2} s_{K}\left(x_{\uparrow \downarrow}\right)
\end{array}
$$

onde

$$
s_{K}\left(x_{\uparrow \downarrow}\right)=\left(\mathrm{e}^{i K_{x}} \delta_{x_{\uparrow \downarrow},(1,0)}+\mathrm{e}^{-i K_{x}} \delta_{x_{\uparrow \downarrow},(-1,0)}+\mathrm{e}^{-i K_{y}} \delta_{x_{\uparrow \downarrow},(0,-1)}+\mathrm{e}^{i K_{y}} \delta_{x_{\uparrow \downarrow},(0,1)}\right)
$$

temos que

$$
\phi_{f}\left(x_{\uparrow}, x_{\downarrow}\right)=\frac{4 \varepsilon^{2}}{(2 \pi)^{2}} \frac{\varkappa}{E_{0}} \sum_{K \in k_{m}} \mathrm{e}^{i K \cdot x_{\uparrow}}\left(\frac{1}{1+U_{0} / E_{0}} \delta_{x_{\uparrow}, x_{\downarrow}}+\mathrm{e}^{-\kappa} s_{K}\left(x_{\downarrow}-x_{\uparrow}\right)\right)
$$

Para considerar os limites $U_{0} \rightarrow 0$ e $U_{0} \rightarrow \infty$, na função do par de elétrons, temos que ter em conta que $E_{0}$ depende de $U_{0}$, então usando a equação (2.22) temos que, se $\epsilon$ tende a zero

$$
\begin{gathered}
|\hat{v}(K)|^{2} \mathcal{R}(K, \lambda)+\lambda-\epsilon h_{b}(2-\cos (K)) \rightarrow|\hat{v}(K)|^{2} \mathcal{R}(K, \lambda)+\lambda \rightarrow 0 \\
\lambda \rightarrow-\varkappa^{2} \mathcal{R}(K, \lambda) \\
\mathcal{R}(K, \lambda)=\frac{\mathfrak{R}_{\mathfrak{f}, \mathfrak{f}}^{(0)}}{1+U_{0} \mathfrak{R}_{0,0}^{(0)}}+U_{0} \frac{\mathfrak{R}_{\mathfrak{f}, \mathfrak{f}}^{(0)} \mathfrak{R}_{0,0}^{(0)}-\left(\mathfrak{R}_{0, \mathfrak{f}}^{(0)}\right)^{2}}{1+U_{0} \mathfrak{R}_{0,0}^{(0)}}
\end{gathered}
$$


se $U_{0} \rightarrow 0$ e $\epsilon \rightarrow 0$

$\mathcal{R}(K, \lambda) \rightarrow \mathfrak{R}_{\mathfrak{f}, \mathfrak{f}}^{(0)}=\left\langle 1+2 \mathrm{e}^{-\kappa} \cos \left(k_{\uparrow \downarrow}-K\right),\left(A_{1,1}^{(0)}(K)-\lambda\right)^{-1}\left(1+2 \mathrm{e}^{-\kappa} \cos \left(k_{\uparrow \downarrow}-K\right)\right)\right\rangle \rightarrow-\frac{1+4 \mathrm{e}^{-2 \kappa}}{\lambda}$

o que implica que

$$
E_{0}=\lambda \rightarrow-\varkappa \sqrt{1+4 \mathrm{e}^{-2 \kappa}} \doteq E_{0}^{(0)}
$$

Agora se $U_{0} \rightarrow \infty$ e $\epsilon \rightarrow 0$

$$
\mathcal{R}(K, \lambda) \rightarrow \frac{R_{\mathfrak{f}, \mathfrak{f}}^{(0)} \mathfrak{R}_{0,0}^{(0)}-\left(\mathfrak{R}_{0, \mathfrak{f}}^{(0)}\right)^{2}}{\mathfrak{R}_{0,0}^{(0)}} \rightarrow-\frac{4 \mathrm{e}^{-2 \kappa}}{\lambda}
$$

o que implica que

$$
E_{0}=\lambda \rightarrow-2 \varkappa \mathrm{e}^{-\kappa} \doteq E_{0}^{(\infty)}
$$

Calculados os limites da energia $E_{0}$ quando $U_{0} \rightarrow 0$ e $U_{0} \rightarrow \infty$ temos que

$$
\phi_{f}\left(x_{\uparrow}, x_{\downarrow}\right) \rightarrow \frac{4 \varepsilon^{2}}{(2 \pi)^{2}} \frac{\varkappa}{E_{0}^{(0)}} \sum_{K \in k_{m}} \mathrm{e}^{i K \cdot x_{\uparrow}}\left(\delta_{x_{\uparrow}, x_{\downarrow}}+\mathrm{e}^{-\kappa} s_{K}\left(x_{\downarrow}-x_{\uparrow}\right)\right) \quad \text { se } U_{0} \rightarrow 0
$$

e

$$
\phi_{f}\left(x_{\uparrow}, x_{\downarrow}\right) \rightarrow \frac{4 \varepsilon^{2}}{(2 \pi)^{2}} \frac{\varkappa}{E_{0}^{(\infty)}} \sum_{K \in k_{m}} \mathrm{e}^{i K \cdot x_{\uparrow}} \mathrm{e}^{-\kappa} s_{K}\left(x_{\downarrow}-x_{\uparrow}\right) \quad \text { se } U_{0} \rightarrow \infty .
$$

Observamos que no limite $U_{0} \rightarrow \infty$ as componentes da função de onda $\phi_{f}\left(x_{\uparrow}, x_{\downarrow}\right)$ associadas a coordenada relativa dos dois elétrons $s_{(0, \pi)}\left(x_{\downarrow}-x_{\uparrow}\right), s_{(0,-\pi)}\left(x_{\downarrow}-x_{\uparrow}\right)$, $s_{(\pi, 0)}\left(x_{\downarrow}-x_{\uparrow}\right)$ e $s_{(-\pi, 0)}\left(x_{\downarrow}-x_{\uparrow}\right)$ tem simetria d-wave, o que fica mais evidente ainda na sua transformada de Fourier

$$
\hat{s}_{(0, \pi)}=\cos \left(K_{x}\right)-\cos \left(K_{y}\right)
$$

Temos então que no limite $U_{0} \rightarrow \infty$ a onda de os férmions é d-wave pura. Para repulsões pequenas, $U_{0} \rightarrow 0$, os elétrons tem mais probabilidade de ficar num mesmo sitio da rede e a onda tem componente s-wave. Como vimos este comportamento acontece se o termo cinético da energia é muito pequeno, e a função de interação $\hat{v}(K)$ tem picos muito 
localizados nos puntos $K \in k_{m}$.

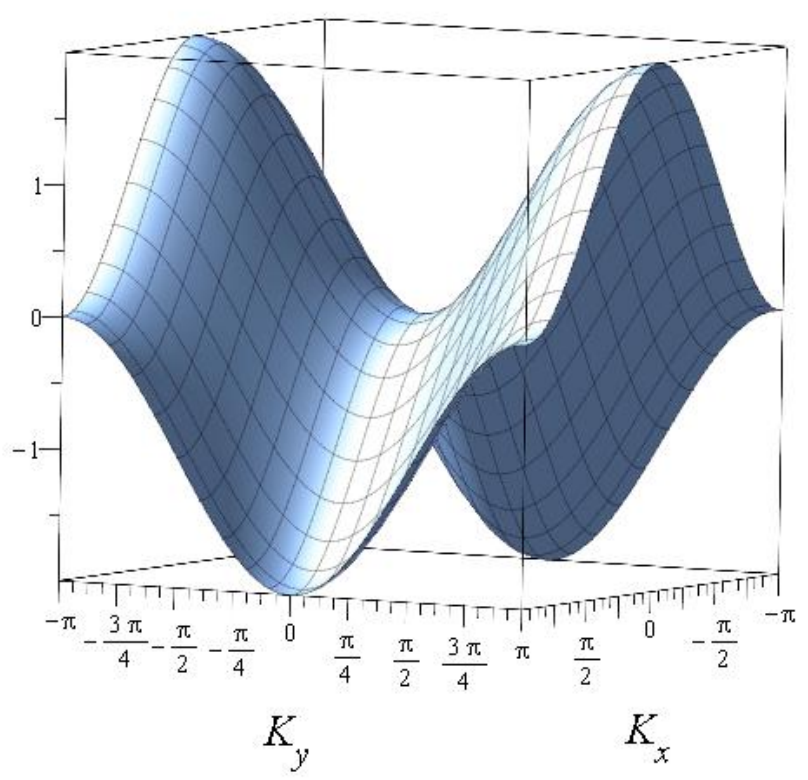

Figura 2.6: Gráfico da função d-wave $\hat{s}_{(0, \pi)}$. 


\section{Capítulo 3}

\section{Efeitos do Alcance da Repulsão}

\section{Eletrônica e da Interação}

\section{Bipolaron-Elétron sobre a Simetria}

\section{dos Pares}

No capítulo anterior vimos que se levamos em conta somente a repulsão local na Hamiltoniana (2.1), o limite $\epsilon \rightarrow 0$ e a função $\hat{v}(K)$ muito localizada nos pontos $(0, \pm \pi),( \pm \pi, 0)$ obtemos que o parâmetro de ordem tem simetria d-wave pura no limite em que a repulsão coulombiana é muito forte. Surge a pergunta: o que aconteceria no caso com repulsão não local? O tratamento analítico para o caso não local é mais difícil, devido a que ao aumentar o alcance da repulsão o calculo direto da energia do estado base é inviável.

Outro efeito que pode considerar-se é o devido ao alcance da interação bipolaronelétron, que é parametrizada por $\gamma \kappa^{-1}$ como pode ser visto na equação (2.4). Anteriormente consideramos só $\gamma \kappa^{-1}=1$ por simplicidade no tratamento analítico. Nos cálculos numéricos exatos presentados aqui consideramos também o caso $\gamma \kappa^{-1}>1$.

Estamos interessados no perceber o efeito dos alcances da repulsão e a interação de acoplamento sobre a simetria do parâmetro de ordem no limite "strong coupling", 
ainda assim aqui consideramos os cálculos exatos para $\epsilon \neq 0$ no termo cinético do nosso modelo. Também consideramos o caso no que a função de acoplamento $\hat{v}(K)$ tem seus máximos fora dos pontos $K \in\{( \pm \pi, 0),(0, \pm \pi)\}$, em particular consideramos $K \in\{( \pm \pi / 2,0),(0, \pm \pi / 2)\}$ e mostramos que ao considerar esta forma para a interação surge uma componente $p$-wave na função do par fermiônico.

\section{Estudo Numérico de Termos de Repulsão não Locais}

A correspondente solução da equação de Schrödinger no espaço invariante $\mathcal{H}_{\uparrow, \downarrow}^{(2,1)}$ será analisada por métodos numéricos. Nos gráficos que presentamos agora representamos a componente simétrica ( $s$-wave) e a componente antissimétrica ( $d$-wave) da função de onda na energia do estado fundamental. Observamos nos gráficos que, em geral, a função de onda não é completamente antissimétrica no limite $U_{0} / \varkappa>>0$.

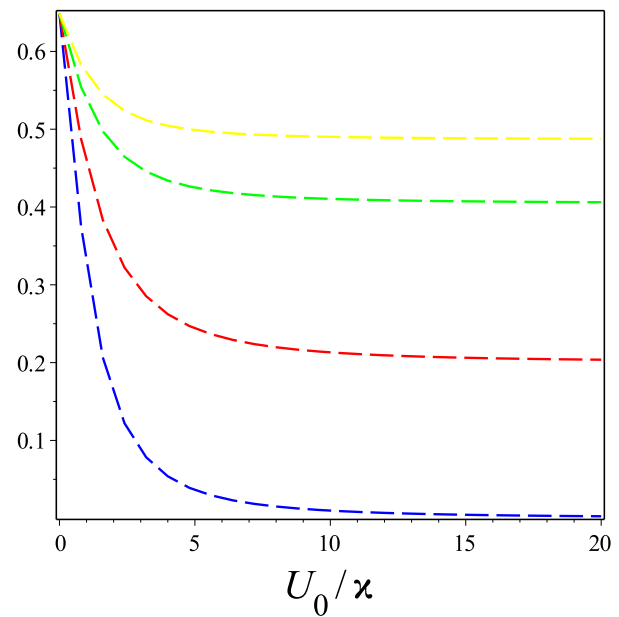

(a) componente s-wave

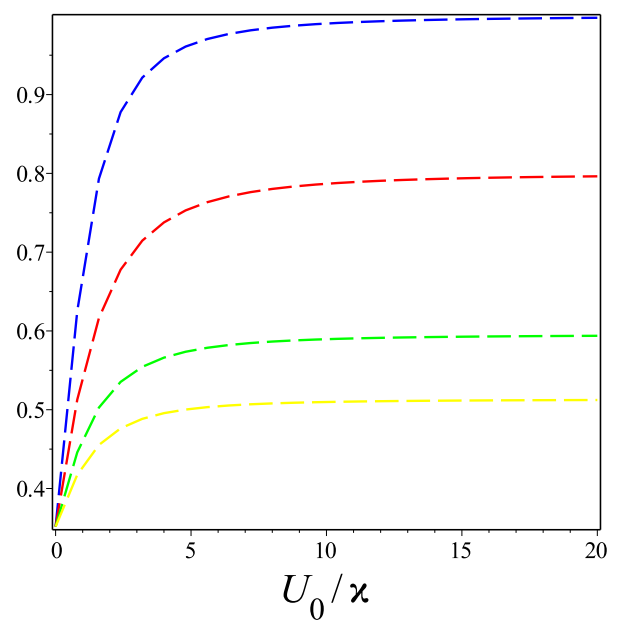

(b) componente d-wave

Figura 3.1: $U_{z}=U_{0} \mathrm{e}^{-\frac{|z|}{m}} \quad \kappa=\frac{1}{n} \quad \gamma=1 \quad k_{x}=0 \quad k_{y}=\pi \quad \varkappa=5 \quad \epsilon=0.01 \quad h_{b}=$ $0.2 n=1 \quad m=0^{+}$azul $m=1$ vermelho $m=2$ verde $m=3$ amarelo.

Observamos também nos gráficos acima que a componente d-wave decresce quando o alcance da repulsão eletrônica cresce. A formação de pares $d$-waves puros (no limite $\left.U_{0} / \varkappa>>0\right)$ se apresenta só no caso no qual o "tamanho dos bipolarons" $\left(\gamma \kappa^{-1}=1\right)$ é maior que o alcance da repulsão (ver (2.4)). 
Para confirmar a hipótese de que a componente $d$-wave domina só no caso no que o tamanho tipico do bipolaron é maior que o alcance da repulsão, no seguente cálculo numérico nos fixamos o alcance da repulsão $m=3$ e consideramos $(\gamma, \kappa)=(1,1 / n)$ para $n=1,2,3,4$ e 5 . Neste cálculo escolhemos uma pequena amplitude do termo cinético $(\epsilon=0.01)$ devido ao interesse no regime de forte acoplamento e a que a simetria do par fermiônico esta mais relacionado a uma competição entre os alcances da interação bipolaron-elétron e a repulsão entre elétrons como se observou anteriormente.

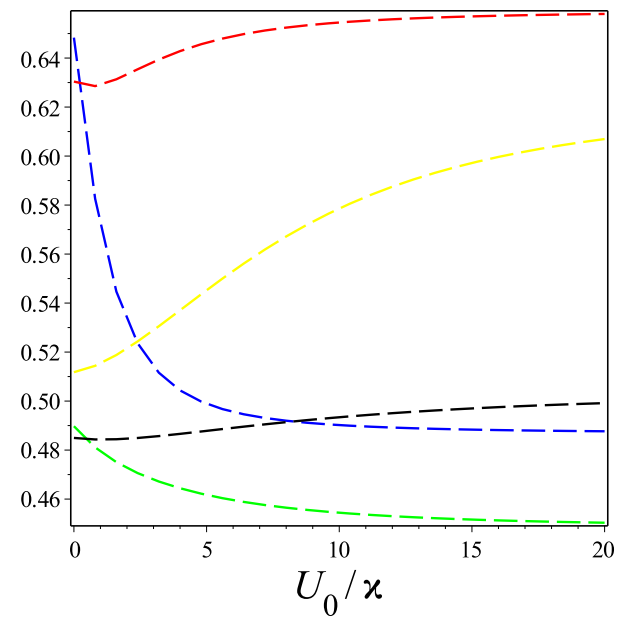

(a) componente s-wave

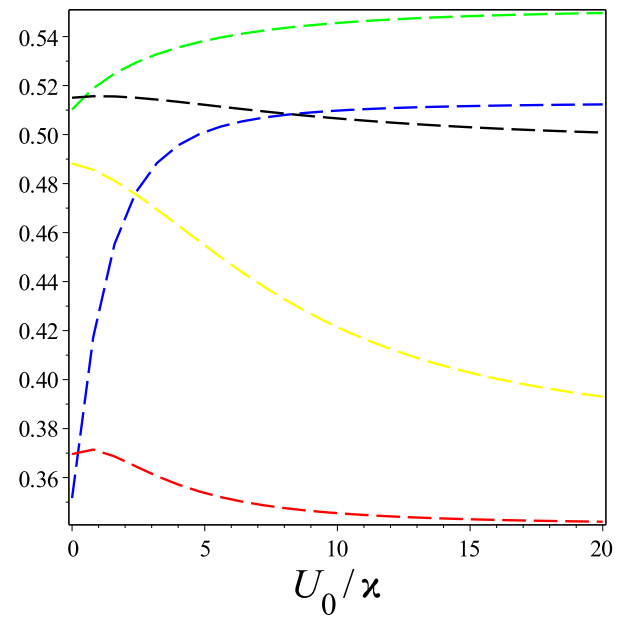

(b) componente d-wave

Figura 3.2: $U_{z}=U_{0} e^{-\frac{|z|}{m}} \quad \kappa=\frac{1}{n} \quad \gamma=1 \quad k_{x}=0 \quad k_{y}=\pi \quad \varkappa=5 \quad \epsilon=0.01 \quad h_{b}=$ $0.2 m=3 \quad n=1$ azul $n=2$ vermelho $n=3$ verde $n=4$ amarelo $n=5$ preto.

Por uma inspeção direta dos gráficos acima, observamos que a componente antissimétrica ou $d$-wave, é maximizada para $\gamma \kappa^{-1}=m$ (curva verde). Se interpretamos novamente a $\gamma \kappa^{-1}$ como o tamanho do bipolaron e $m$ como o alcance da repulsão efetiva, a condição física para ter uma simetria que tenha mais componente d-wave seria que o alcance da repulsão fosse igual que o tamanho típico do bipolaron. Notamos então que a componente $d$-wave e máxima (no limite $U_{0} / \varkappa>>0$ ) quando $\gamma \kappa^{-1}=m$ e também que quando $\gamma \kappa^{-1}=m-1$ a componente $d$-wave é minimizada (curva vermelha), isto sugere um comportamento singular no caso em que o tamanho do bipolaron é comparável ao alcance da repulsão eletrônica. 
Agora consideramos o caso $(\gamma, \kappa)=(3,1)$ com $m=0,1,2$ e 3 .

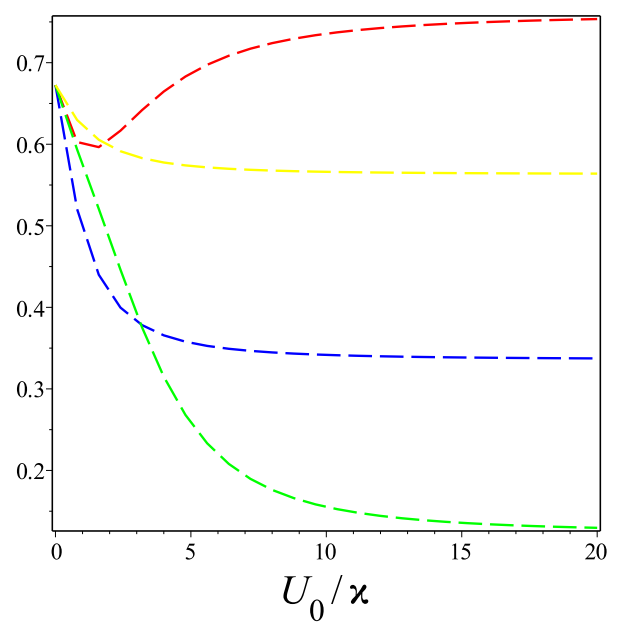

(a) componente s-wave

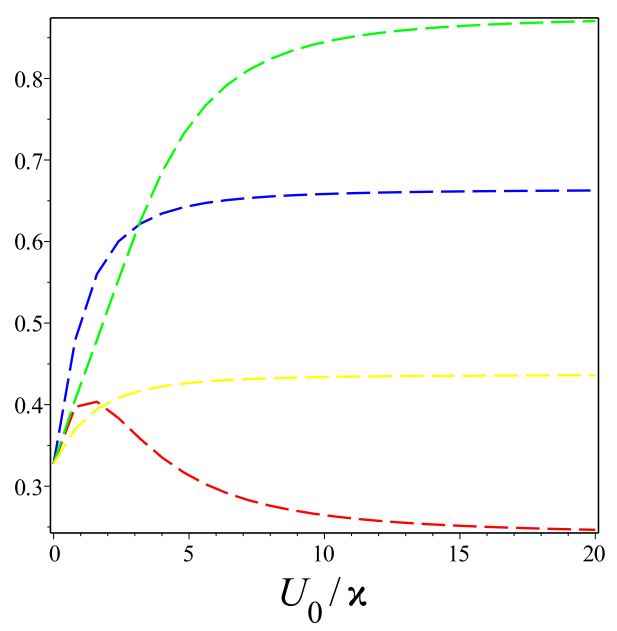

(b) componente d-wave

Figura 3.3: $U_{z}=U_{0} e^{-\frac{|z|}{m}} \quad \kappa=\frac{1}{n} \quad \gamma=3 \quad k_{x}=0 \quad k_{y}=\pi \quad \varkappa=5 \quad \epsilon=0.01 \quad h_{b}=$ $0.2 n=1 \quad m=0^{+}$azul $m=1$ vermelho $m=2$ verde $m=3$ amarelo.

Observamos nos gráficos acima coerentemente com nossa hipótese anterior que neste caso a componente $d$-wave é maximizada para $m=2$, ou seja quando o tamanho do bipolaron $\left(\gamma \kappa^{-1}=3\right)$ é maior que o alcance $(m=2)$ da repulsão. 


\section{Considerações para o Calculo das Componentes de Função de Onda e Simetria $p$-wave}

Como vimos no capítulo anterior a autofunção da fibra associada ao impulso total $K$ do Hamiltoniano $H^{(2,1)}$ para um $m \geq 0$ e $\gamma \kappa^{-1} \geq 1$ é

$$
\Psi_{\uparrow \downarrow}=-\left(A_{1,1}^{(0)}(K)-\lambda\right)^{-1} \hat{v}(K)\left(\mathfrak{f}-\sum_{|z| \leq m} U_{z} e^{-i k_{\uparrow \downarrow} \cdot z} \mathfrak{R}_{z, \mathfrak{f}}\right)
$$

onde

$$
\left(A_{1,1}^{(0)}(K)-\lambda\right)^{-1}=\frac{1}{\epsilon\left(4-\cos \left(k_{\uparrow \downarrow}-K\right)-\cos \left(k_{\uparrow \downarrow}\right)\right)-\lambda}
$$

e o elemento de matriz

$$
\mathfrak{R}_{z, \mathfrak{f}}=\left\langle\mathrm{e}^{-i k_{\uparrow \downarrow} \cdot z},\left(A_{1,1}(K)-\lambda\right)^{-1} \mathfrak{f}\right\rangle
$$

com

$$
\mathfrak{f}=1+\left[f^{(\gamma, \kappa)}(K)\right]\left(k_{\uparrow \downarrow}\right)
$$

e

$$
\left[f^{(\gamma, \kappa)}(K)\right]\left(k_{\uparrow \downarrow}\right)=2 \sum_{0<n^{\prime} \leq \gamma \kappa^{-1}} \mathrm{e}^{-\kappa n^{\prime}} \cos \left(n^{\prime}\left(k_{\uparrow \downarrow}-K\right)\right)+4 \sum_{n^{\prime}, m^{\prime}>0} \sum_{\left|z^{\prime}\right|<\gamma \kappa^{-1}} e^{-\kappa\left|z^{\prime}\right|}\left[g_{n^{\prime}, m^{\prime}}(K)\right]\left(k_{\uparrow \downarrow}\right)
$$

onde a função $\left[g_{n^{\prime}, m^{\prime}}(K)\right]\left(k_{\uparrow \downarrow}\right)$ é definida da seguinte forma:

$$
\left[g_{n^{\prime}, m^{\prime}}(K)\right]\left(k_{\uparrow \downarrow}\right):=\cos \left(n^{\prime}\left(k_{\uparrow \downarrow x}-K_{x}\right)\right) \cos \left(m^{\prime}\left(k_{\uparrow \downarrow y}-K_{y}\right)\right) .
$$

Os elementos de matriz $\mathfrak{R}_{z, \mathfrak{f}}$ são calculados a partir da equação (2.27).

Notamos que nos pontos $k^{\prime} \in k_{m}=\{(0, \pi),(0,-\pi),(\pi, 0),(-\pi, 0)\}$ a função $\Psi_{\uparrow \downarrow}$ é invariante sob reflexão, ou seja

$$
\left[\Psi_{\uparrow \downarrow}\left(k^{\prime}\right)\right]\left(-k_{\uparrow \downarrow}\right)=\left[\Psi_{\uparrow \downarrow}\left(k^{\prime}\right)\right]\left(k_{\uparrow \downarrow}\right)
$$


Isto vem do fato que $\left(A_{1,1}^{(0)}\left(k^{\prime}\right)-\lambda\right)^{-1}$ e $\mathfrak{f}$ são invariantes sob reflexão para $k^{\prime} \in k_{m}$ e de que $U_{z}=U_{-z}$, portanto

$$
\sum_{|z| \leq m} U_{z} e^{-i k_{\uparrow \downarrow} \cdot z} R_{z, \mathfrak{f}}=\sum_{|z| \leq m} U_{z} e^{i k_{\uparrow \downarrow} \cdot z} R_{z, \mathfrak{f}}
$$

notamos também que $R_{-z, \mathfrak{f}}=R_{z, \mathfrak{f}} \in \mathbb{R}$, isto a causa de que o operador $\left(A_{1,1}(K)-\lambda\right)^{-1}$ atua sobre $\mathfrak{f}$, que é invariante sob reflexão (ou par) nos pontos $K \in\{( \pm \pi, 0),(0, \pm \pi)\}$. Como o operador $\left(A_{1,1}(K)-\lambda\right)$ conserva a paridade (para $\left.K \in\{( \pm \pi, 0),(0, \pm \pi)\}\right)$ da função sobre a que atua, isto implica que seu inverso $\left(A_{1,1}(K)-\lambda\right)^{-1}$ também conservara a paridade.

Como vimos anteriormente o espaço (fibra) de um par de férmions em quasimomentum constante $K$ é o Espaço de Hilbert $L^{2}\left(\mathbb{T}^{2} ; \mathbb{C}\right)$ com produto escalar

$$
\langle\varphi \mid \psi\rangle \doteq \frac{1}{(2 \pi)^{2}} \int_{\mathbb{T}^{2}} \overline{\varphi(k)} \psi(k) \mathrm{d}^{2} k
$$

Usamos agora a notação bra-ket para elementos deste espaço. Assim denotamos um elemento deste espaço como $|\varphi\rangle$, mas a função $|\varphi\rangle$ em um $K \in \mathbb{T}^{2}$ é escrito como $\varphi(K) \in \mathbb{C}$.

A rede é invariante sob o grupo $\{0, \pi / 2, \pi, 3 \pi / 2\}$ de rotações, que é gerada pelas $\pi / 2$-rotações. Definimos por

$$
\left[R_{\perp}|\varphi\rangle\right]\left(k_{x}, k_{y}\right) \doteq \varphi\left(k_{y},-k_{x}\right), \quad\left(k_{x}, k_{y}\right) \in \mathbb{T}^{2}
$$

o operador unitário $R_{\perp}$ implementa a $\pi / 2$-rotação em $L^{2}\left(\mathbb{T}^{2} ; \mathbb{C}\right)$. Definimos também os 
projetores mutuamente ortogonais ${ }^{1}$

$$
\begin{aligned}
P_{s} & \doteq \frac{R_{\perp}^{4}+R_{\perp}^{3}+R_{\perp}^{2}+R_{\perp}}{4} \\
P_{d} & \doteq \frac{R_{\perp}^{4}-R_{\perp}^{3}+R_{\perp}^{2}-R_{\perp}}{4} \\
P_{p} & \doteq \frac{R_{\perp}^{4}-R_{\perp}^{2}}{2}
\end{aligned}
$$

Desde que $P_{s}+P_{d}+P_{p}=\mathbf{1}$, qualquer função de onda $|\varphi\rangle \in L^{2}\left(\mathbb{T}^{2} ; \mathbb{C}\right)$ de um par de férmions pode ser decomposta em componentes ortogonais $s, d$-e $p$-wave como

$$
|\varphi\rangle=\left|\varphi^{s}\right\rangle+\left|\varphi^{d}\right\rangle+\left|\varphi^{p}\right\rangle, \quad\left|\varphi^{\#}\right\rangle \doteq P_{\#}|\varphi\rangle
$$

Observe que

$$
R_{\perp}\left|\varphi^{s}\right\rangle=\left|\varphi^{s}\right\rangle, \quad R_{\perp}\left|\varphi^{d}\right\rangle=-\left|\varphi^{d}\right\rangle, \quad R_{\perp}^{2}\left|\varphi^{p}\right\rangle=-\left|\varphi^{p}\right\rangle
$$

Em particular, se $|\varphi\rangle \in L^{2}\left(\mathbb{T}^{2} ; \mathbb{C}\right)$ é invariante em relação a $R_{\perp}^{2}$, então $\left|\varphi^{p}\right\rangle$ desaparece de forma idêntica e $|\varphi\rangle$ tem apenas $s$ - e $d$-wave componentes.

A simetria $p$-wave é observada também no parâmetro de ordem de alguns supercondutores. No nosso modelo não aparece a componente $p$-wave por causa da localização dos picos da função $\hat{v}(K)$. Se consideramos que os picos estiveram deslocados

\footnotetext{
${ }^{1}$ Para encontrar expressões explícitas destes projetores em termos de $R_{\perp}$ observamos que
}

$$
R_{\perp} P_{s}|\varphi\rangle=\left|\varphi_{s}\right\rangle \text { e } R_{\perp} P_{d}|\varphi\rangle=-\left|\varphi_{d}\right\rangle .
$$

Para o projetor $P_{s}$

$$
(R_{\perp}+R_{\perp}^{2}+R_{\perp}^{3}+\underbrace{R_{\perp}^{4}}_{\mathbb{1}}) P_{s}|\varphi\rangle=4\left|\varphi_{s}\right\rangle .
$$

O operador

$$
\widetilde{P}=\frac{R_{\perp}+R_{\perp}^{2}+R_{\perp}^{3}+\mathbb{1}}{4}
$$

é autoadjunto e idempotente, portanto um projetor ortogonal. Vemos então que

$$
\widetilde{P} P_{s}=P_{s}^{2} \Rightarrow \widetilde{P}=P_{s} .
$$

De forma similar vemos que

$$
P_{d}=\frac{-R_{\perp}+R_{\perp}^{2}-R_{\perp}^{3}+\mathbb{1}}{4} .
$$


dos pontos $(0, \pm \pi),( \pm \pi, 0)$, fazendo a mesma análise podemos observar também uma componente $p$-wave na função de onda do par de férmions. Apresentamos agora alguns cálculos numéricos onde a função $\hat{v}(K)$ tem máximos fora de $(0, \pm \pi),( \pm \pi, 0)$, em particular consideramos que os máximos estejam localizados agora nos pontos $(0, \pm \pi / 2),( \pm \pi / 2,0)$.

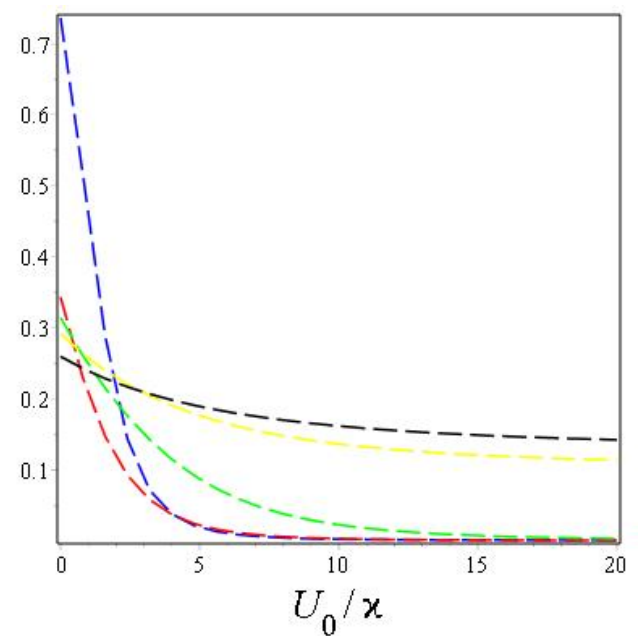

(a) componente s-wave

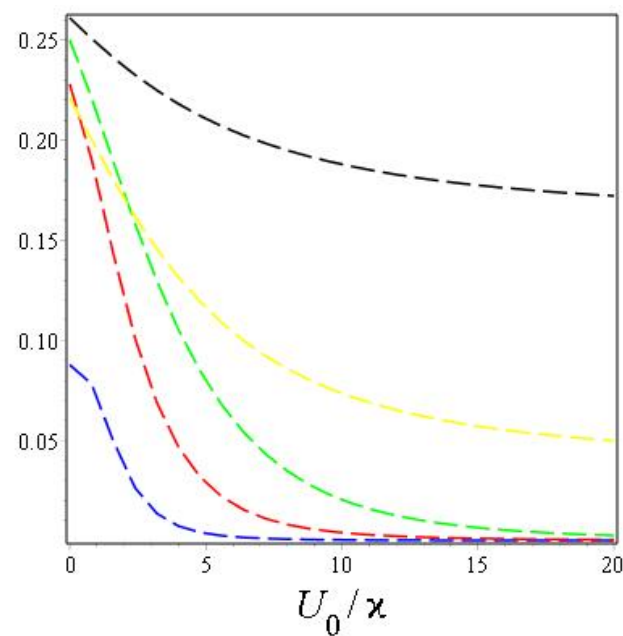

(b) componente d-wave

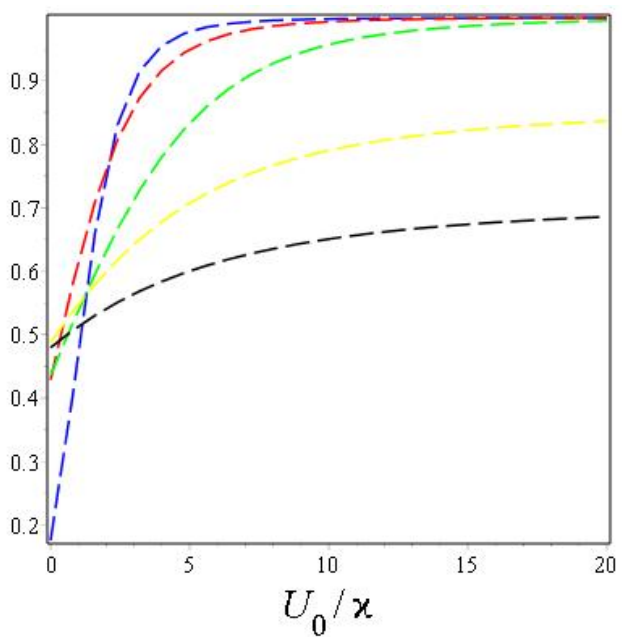

(c) componente p-wave

Figura 3.4: $U_{z}=U_{0} \mathrm{e}^{-\frac{|z|}{m}} \quad \kappa=\frac{1}{n} \quad \gamma=1 \quad k_{x}=0 \quad k_{y}=\pi / 2 \quad \varkappa=5 \quad \epsilon=0.01 \quad h_{b}=$ $0.2 m=3 \quad n=1$ azul $n=2$ vermelho $n=3$ verde $n=4$ amarelo $n=5$ preto. 


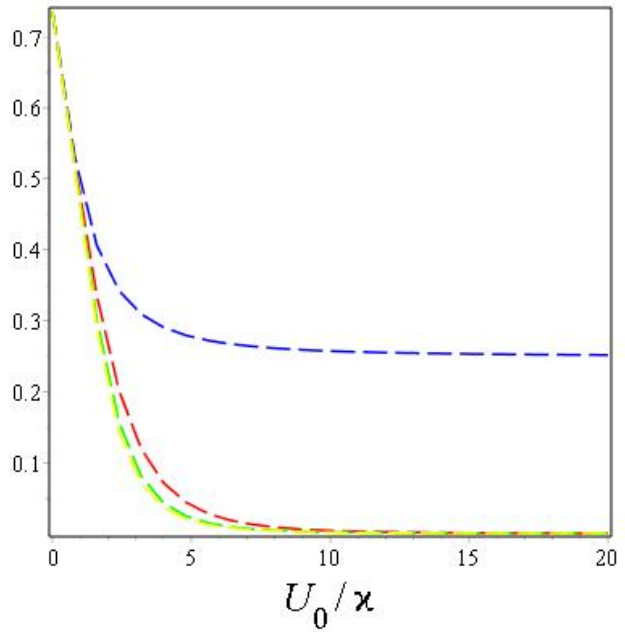

(a) componente s-wave

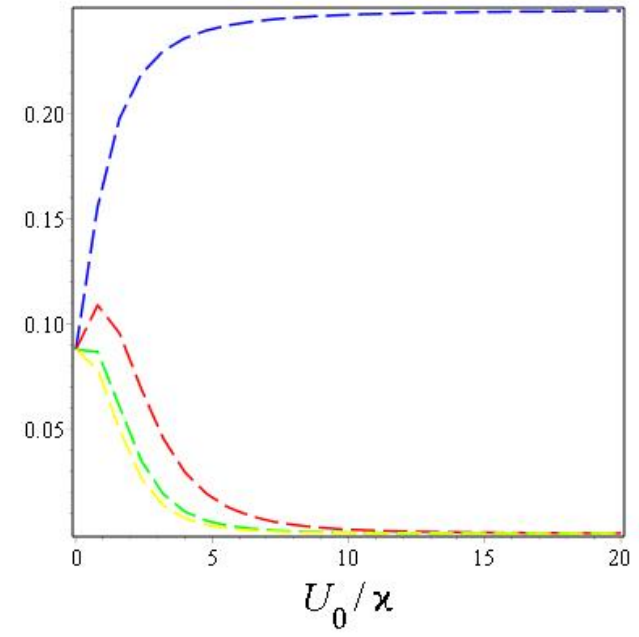

(b) componente d-wave

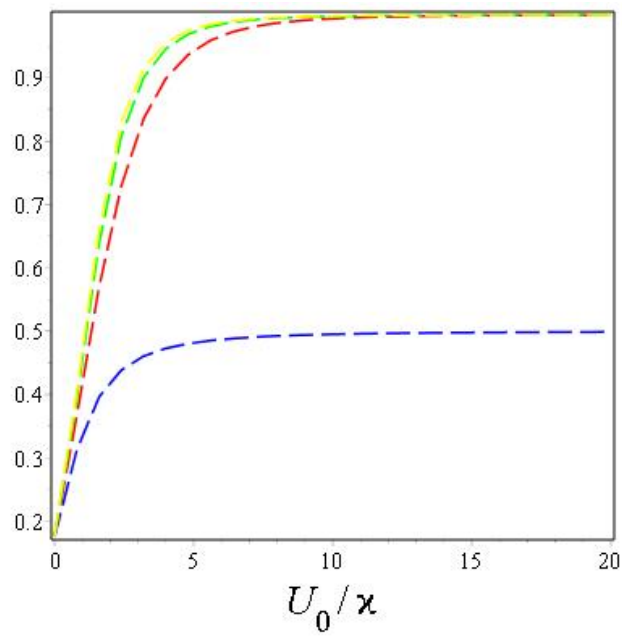

(c) componente p-wave

Figura 3.5: $U_{z}=U_{0} \mathrm{e}^{-\frac{|z|}{m}} \quad \kappa=\frac{1}{n} \quad \gamma=1 \quad k_{x}=0 \quad k_{y}=\pi / 2 \quad \varkappa=5 \quad \epsilon=0.01 \quad h_{b}=$ $0.2 n=1 \quad m=0^{+}$azul $m=1$ vermelho $m=2$ verde $m=3$ amarelo. 


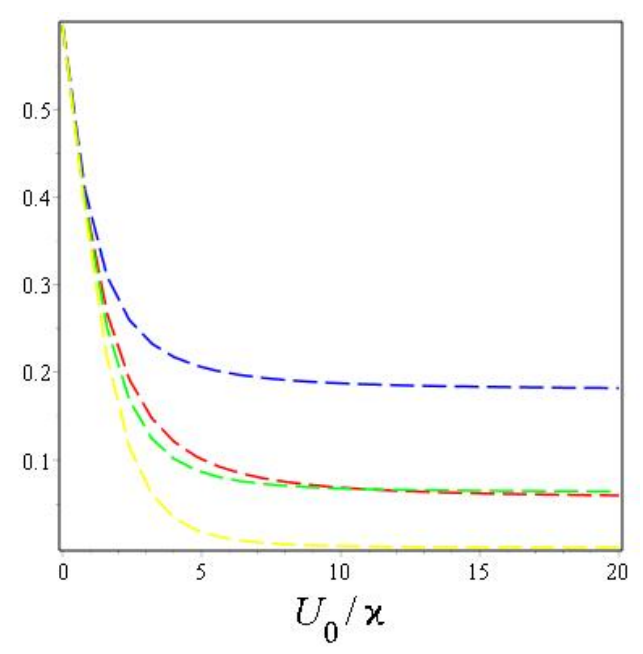

(a) componente s-wave

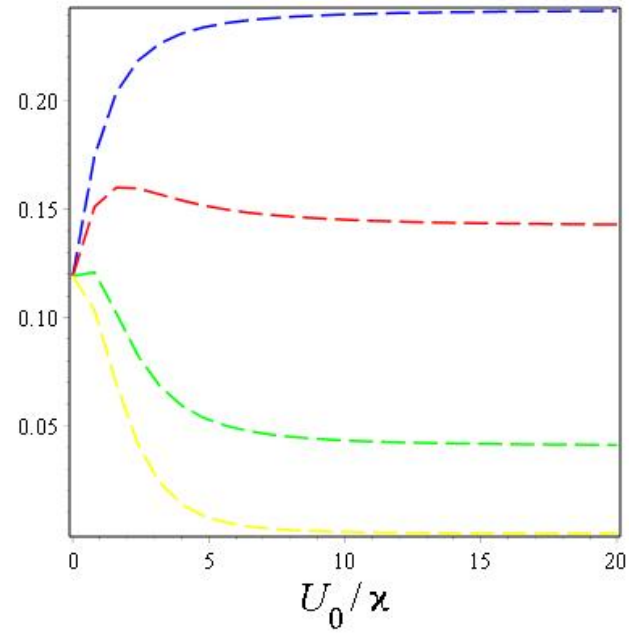

(b) componente d-wave

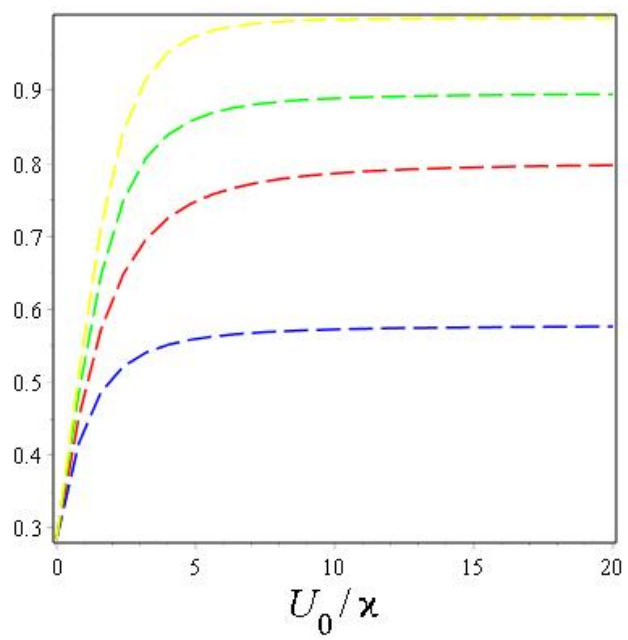

(c) componente p-wave

Figura 3.6: $U_{z}=U_{0} \mathrm{e}^{-\frac{|z|}{m}} \quad \kappa=\frac{1}{n} \quad \gamma=3 \quad k_{x}=0 \quad k_{y}=\pi / 2 \quad \varkappa=5 \quad \epsilon=0.01 \quad h_{b}=$ $0.2 n=1 \quad m=0^{+}$azul $m=1$ vermelho $m=2$ verde $m=3$ amarelo.

Observamos nos gráficos anteriores que a formação de pares $p$-wave é favorecida se o alcance da repulsão $(m)$ é grande comparado com o tamanho do bipolaron $\left(\gamma \kappa^{-1}\right)$. Os cálculos sugerem que no contexto da formação de pares mediadas por bipolarons, a existência de pares $p$-wave esta relacionada ao fato de que a função de acoplamento da interação bipolaron-elétron $\hat{v}(K)$ é concentrada longe dos pontos $K \in\{(0, \pm \pi),( \pm \pi, 0)\}$. Isto representa uma explicação teórica para a formação de pares p-wave. 


\section{Capítulo 4}

\section{Ajuste do Modelo Bipolaron-Elétron para o Caso do $\mathrm{La}_{2} \mathrm{CuO}_{4}$}

Com o propósito de testar nosso modelo para o caso particular do $\mathrm{La}_{2} \mathrm{CuO}_{4}$ neste capitulo ajustaremos os parâmetros do Hamiltoniano para conseguir aproximar-nos dos dados experimentais para este material.

Basicamente faremos algumas mudanças pequenas na forma da repulsão e a interação bipolaron-elétron apresentadas anteriormente. Se parametrizara de forma independente a repulsão coulombiana local e não local e para a interação bipolaron-elétron se considerara uma estrutura mais próxima aos dados experimentais para o caso particular do $\mathrm{La}_{2} \mathrm{CuO}_{4}$.

Definimos agora o Hamiltoniano total, similar ao apresentado em (2.5). Para qualquer amplitude de hopping $\epsilon \geq 0$, parâmetros $U, U_{0} \geq 0$, alcance $r \geq 0$ e amplitude da interação bipolaron-elétron $\varkappa \geq 0$, temos

$$
\mathbf{H} \doteq \epsilon \mathbf{T}+U_{0} \mathbf{W}_{\mathrm{f}-\mathrm{f}}^{(0)}+U \mathbf{W}_{\mathrm{f}-\mathrm{f}}^{(r)}+\varkappa \mathbf{W}_{\mathrm{b}-\mathrm{f}}
$$

onde $\mathbf{W}_{\mathrm{f}-\mathrm{f}}^{(0)}, \mathbf{W}_{\mathrm{f}-\mathrm{f}}^{(r)}$ são a repulsão no mesmo site (local) e a repulsão eletrônica fora de mesmo site respetivamente. $\mathrm{O}$ termo $\mathbf{W}_{\mathrm{b}-\mathrm{f}}$ corresponde a interação bipolaron-elétron e o 
termo cinético T fica igual ao usado anteriormente em (2.1) e (2.2)

$$
\begin{aligned}
\mathbf{T} \doteq h_{b}\left(-\frac{1}{2} \sum_{x, y \in \mathbb{Z}^{2},|x-y|=1} b_{x}^{\dagger} b_{y}\right. & \left.+2 \sum_{x \in \mathbb{Z}^{2}} b_{x}^{\dagger} b_{x}\right) \\
& -\frac{1}{2} \sum_{s \in\{\uparrow, \downarrow\}, x, y \in \mathbb{Z}^{2},|x-y|=1} a_{x, s}^{\dagger} a_{y, s}+2 \sum_{s \in\{\uparrow, \downarrow\}, x \in \mathbb{Z}^{2}} a_{x, s}^{\dagger} a_{x, s}
\end{aligned}
$$

Aqui como foi definido anteriormente, $h_{b} \geq 0$ é a razão entre a amplitude de "hopping" dos bipolarons e a dos férmions. Este parâmetro fixa a relação entre as massas desses dois tipos de partícula.

Para $r \geq 0$ e $\lambda>0$, a repulsão coulombiana parcialmente blindada dos férmions é definida pelas interações densidade-densidade definidas por ${ }^{1}$

$$
\begin{aligned}
& \mathbf{W}_{\mathrm{f}-\mathrm{f}}^{(0)} \doteq \sum_{\substack{x \in \mathbb{Z}^{2}, s_{1}, s_{2} \in\{\uparrow, \downarrow\} \\
\mathbf{W}_{\mathrm{f}-\mathrm{f}}^{(r)}}} a_{x, s_{1}}^{\dagger} a_{x, s_{1}} a_{x, s_{2}}^{\dagger} a_{x, s_{2}} . \\
& \sum_{\substack{x, z \in \mathbb{Z}^{2}, 1 \leq|z| \leq r \\
s_{1}, s_{2} \in\{\uparrow, \downarrow\}}} \mathrm{e}^{-\lambda^{-1}|z|} a_{x, s_{1}}^{\dagger} a_{x, s_{1}} a_{x+z, s_{2}}^{\dagger} a_{x+z, s_{2}}
\end{aligned}
$$

O termo correspondente a interação bipolaron-elétron é definida como antes (salvo a forma do operador $c_{x}$ ) por

$$
\mathbf{W}_{\mathrm{b}-\mathrm{f}} \doteq \sum_{x, y \in \mathbb{Z}^{2}} v(x-y)\left(b_{x}^{\dagger} c_{y}+c_{y}^{\dagger} b_{x}\right)
$$

\footnotetext{
${ }^{1}$ Ressaltamos que a diferença do modelo apresentado em (2.1) aqui parametrizamos, desde o começo, de forma independente a repulsão local e a não local. Mas aclaramos que na verdade o Hamiltoniano (2.1) contempla este caso definindo

$$
U_{z} \doteq U \mathrm{e}^{-\lambda^{-1}|z|}+\left(U_{0}-U\right) \delta_{0,|z|}, \quad z \in \mathbb{Z}^{2}
$$

a diferença da função

$$
U_{z}=U_{0} \mathrm{e}^{-\frac{|z|}{m}}
$$

escolhida anteriormente para o estudo numérico que foi feito.
} 
$\operatorname{com} v: \mathbb{Z}^{2} \rightarrow \mathbb{R}$ uma função igual à definida anteriormente em (2.29), e

$$
c_{x} \doteq \sum_{z \in \mathbb{Z}^{2},|z| \leq 1}\left(a_{x+z, \uparrow} a_{x, \downarrow}+a_{x+z, \uparrow} a_{x-z, \downarrow}\right)
$$

é o operador de aniquilação de o par fermiônico perto do $x \in \mathbb{Z}^{2}$. Aqui notamos a diferença respeito ao operador $c_{x}$ definido em (2.4). A seguir justificamos esta escolha.

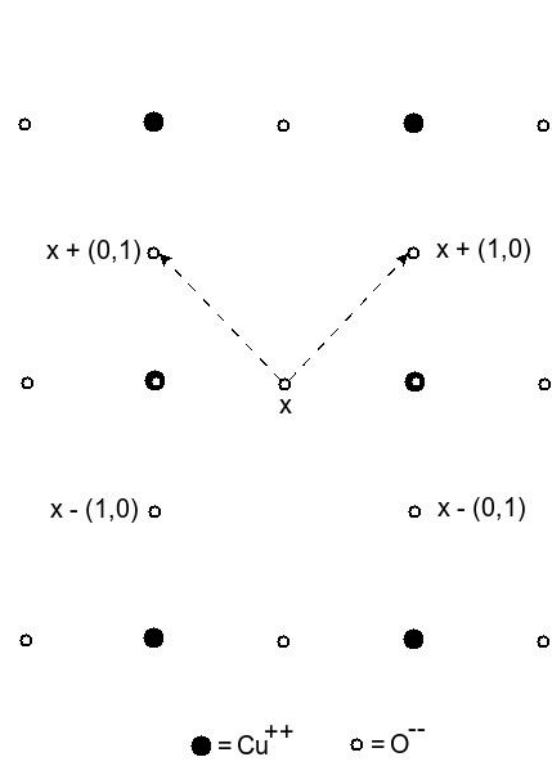

Figura 4.1: $\mathrm{CuO}_{2}$ layer.

A forma escolhida para $c_{x}$ é baseada na estrutura do bipolarons de Jahn-Teller obtida de cálculos $a b$ initio para $\mathrm{La}_{2} \mathrm{CuO}_{4}$ dopados [10], como esta descrito em $[11$, Sect. 5.2]: JT bipolarons são formados por dois buracos antiparalelos presos nos ligantes de oxigênio de dois íons de cobre adjacentes. Cada íon de oxigênio situa-se entre um único par de íons de cobre adjacentes, como esta mostrado na Fig. 4.1. Então podemos associar com cada oxigênio no site da rede $x$ um modo

bipolarônico inter-site. Assumimos que o decaimento de um bipolaron em $x$ produz um par de fermions (buracos nesta discussão) nos ligantes dos íons de cobre correspondentes. Além disso, o par fermiônico deve situar-se entre os íons de cobre e ter seu baricentro próximo a $x$. Essas condições são satisfeitas pelo seguinte espaço configurações do par férmions em torno de $x \in \mathbb{Z}^{2}:\left(y_{\uparrow}, y_{\downarrow}\right)=(x, x+z)$ ou $(x-z, x+z)$ com $|z| \leq 1$. Compare com (4.4) e veja a Fig. 4.1. Note que podemos atribuir facilmente pesos diferentes a cada espaço configuração de pares de férmions.

Com base em fatos experimentais, a estrutura microscópica dos cupratos supercondutores é isotrópico. Portanto, a função $v: \mathbb{Z}^{2} \rightarrow \mathbb{R}$ tem que ser invariante 
sob rotações de $\pi / 2$, como foi dito anteriormente, tal que o Hamiltoniano completo $\mathbf{H} e ́$ invariante em relação a esta rotação. No entanto, $\mathbf{W}_{\mathrm{b}-\mathrm{f}} \mathrm{e}$, portanto, $\mathbf{H}$, não conserva o número do férmions, em contraste com a interação elétron-fônon de Fröhlich. Um modelo semelhante a $\mathbf{H}$ para $r=0$ é proposto como vimos anteriormente para explicar a existência de simetria $d$-wave em baixas energias na presença de isotropia espacial.

\section{Parâmetros Prototípicos dos Experimentos}

Em estudos teóricos usando o modelo de Hubbard, os parâmetros "padrão" são $\epsilon \simeq 1$, $U_{0} \simeq 8(\mathrm{eV})$ e $U=0$. Em geral, estes são principalmente derivados de métodos numéricos. Veja, por exemplo, [12, Tabela 7.1.]. Em contraste, neste trabalho a escolha de todos os parâmetros é baseado em dados experimentais para o dopado $\mathrm{La}_{2} \mathrm{CuO}_{4}$ :

- $(\epsilon)$ A amplitude de "hopping" $\epsilon$ é obtida do espaçamento da rede $\mathbf{a}=2.672 \AA[13$, Sect. 6.3.1] dos íons de oxigênio ${ }^{2}$ e a massa efetiva dos buracos $m^{*} \simeq 4 m_{e}$ [14, Fig. 2.] para $\mathrm{La}_{2-x} \mathrm{Sr}_{x} \mathrm{CuO}_{4}$ com $x \in[0,0.2]$, onde $m_{e}$ é a massa de elétrons. Isto corresponde a $\epsilon=\hbar^{2} /\left(m^{*} \mathbf{a}^{2}\right) \simeq 0.266 \mathrm{eV}$. Compare com [12, Tabela 7.1.].

- $\left(U_{0}\right)$ É mostrado experimentalmente que é a banda de oxigênio a que dá origem à supercondutividade. A força de repulsão local $U_{0}$ é assim considerada como sendo a primeira afinidade eletrônica do oxigênio ${ }^{16} \mathrm{O}$, ou seja, a diferença de energia entre ${ }^{16} \mathrm{O}^{-}$(um buraco em o anião de oxigênio ${ }^{16} \mathrm{O}^{--}$) e ${ }^{16} \mathrm{O}$ (dois buracos). De [15], $U_{0} \simeq 1.461 \mathrm{eV}$. Observe que $U_{0} \epsilon^{-1} \simeq 5.5$, o que corresponde ao usual regime "strong coupling" de todos os estudos teóricos anteriores.

- $(U)$ A repulsão entre sites resulta da repulsão de Coulomb parcialmente blindada. Portanto, estabelecemos $U=U_{0}$ como um parâmetro prototípico. A separação da repulsão férmion-férmion local e entre sites é usado para estudar o regime do núcleo duro, o que corresponde a grandes repulsões locais.

\footnotetext{
${ }^{2}$ Isso corresponde a um espaçamento de rede dos íons de cobre igual a $3.779 \AA$.
} 
- $(\lambda)$ De fatos experimentais temos que o comprimento do blindagem de Thomas-Fermi $\lambda_{\mathrm{TF}}$ é comparável ao comprimento de coerência $\xi$ em cupratos supercondutores [16]. Por exemplo, $\lambda_{\mathrm{TF}} \simeq 5 \AA$ para $\mathrm{YBa}_{2} \mathrm{Cu}_{3} \mathrm{O}_{7-\delta}$. Para o dopado $\mathrm{La}_{2} \mathrm{CuO}_{4}$, estimamos este parâmetro, em duas dimensões, através da fórmula bem conhecida $\lambda_{\mathrm{TF}}=\left(4 \pi \varepsilon \times \hbar^{2}\right) /\left(2 m^{*} \mathbf{e}^{2}\right)$ (unidades SI) com e e $\varepsilon$ sendo, respectivamente, a carga eletrônica e a constante dielétrica das camadas $\mathrm{CuO}_{2}[17$, Eqs. (1.21), (5.38) em unidades gaussianas]. Por [18, Sect. VI.C], $\varepsilon \simeq 30 \varepsilon_{0}$ onde $\varepsilon_{0}$ é a constante dielétrica do espaço livre. Isto produz $\lambda=\lambda_{\mathrm{TF}} \simeq 2 \AA$, semelhante ao caso do $\mathrm{YBa}_{2} \mathrm{Cu}_{3} \mathrm{O}_{7-\delta}$. (Observe que alguns resultados matematicamente rigorosos nas interações de Coulomb blindadas (2D) foram recentemente derivadas em [19, Theorem 3.2].)

- $(r)$ Em duas dimensões, o decaimento da repulsão de Coulomb blindada não é exponencial, mas sim polinomial [17, Eq. (5.41)]. Em particular, mesmo que $\lambda_{\mathrm{TF}} \leq \mathbf{a}$, é razoável considerar a repulsão de Coulomb para alguns sites vizinhos. Assim, tomamos o parâmetro $r=2$ como o valor padrão.

- $\left(h_{b}\right)$ Nós deduzimos a amplitude de "hopping" relativo $h_{b}$ através da massa efetiva dos bipolarons, como experimentalmente é determinado em [20]: $m^{* *} \simeq 695 m_{e}$. Isso corresponde a $h_{b}=m^{*} / m^{* *} \simeq 0.00575$. Dado que [20] foi criticado pela massa bipolarônica muito grande (veja, por exemplo, [21]), também consideramos valores maiores de o parâmetro $h_{b}$, até 1 .

- $(\varkappa)$ A força da interação de acoplamento $\varkappa$ é escolhido para obter $90 \%$ de depleção em $(\pi, 0)$ e $(0, \pi)$ : A computação direta usando [22, Fig. 2, Fig. 3 (b)], com o espaçamento de rede a dos íons de oxigênio igual a $2.672 \AA$, e a massa efetiva $m^{*} \simeq 4 m_{e}$ [14, Fig. 2.] dos buracos móveis, para $\mathrm{La}_{2-x} \mathrm{Sr}_{x} \mathrm{CuO}_{4}$ com $x \simeq 0.2$, da uma depleção da densidade dos pares supercondutores aproximadamente igual a 90\%. Na Fig. $4.3, \varkappa \simeq 0.11 \mathrm{eV}$.

- $(\alpha)$ Como é explicado anteriormente, usamos uma função de acoplamento para a interação bipolaron-férmion $v$ cuja transformada de Fourier $\hat{v}$ é concentrada em 
torno de $(\pi, 0)$ e $(0, \pi)$ na zona de Brillouin $\mathbb{T}^{2} \doteq[-\pi, \pi)^{2}$. Semelhante a $[8,9]$, nós escolhemos como foi feito anteriormente $(2.29) \hat{v}$ da forma

$$
\begin{array}{r}
\hat{v}\left(K_{x}, K_{y}\right)=\max \left\{\frac{1}{\alpha\left(\left(K_{x}-\pi\right)^{2}+K_{y}^{2}\right)+1}, \frac{1}{\alpha\left(\left(K_{x}+\pi\right)^{2}+K_{y}^{2}\right)+1},\right. \\
\left.\frac{1}{\alpha\left(K_{x}^{2}+\left(K_{y}-\pi\right)^{2}\right)+1}, \frac{1}{\alpha\left(K_{x}^{2}+\left(K_{y}+\pi\right)^{2}\right)+1}\right\} .
\end{array}
$$

para momentos $\left(K_{x}, K_{y}\right) \in \mathbb{T}^{2}$ perto de $(\pi, 0)$ ou $(0, \pi)$, onde $\alpha>0$. A transformada de Fourier $\hat{v}$ é esboçada em a Fig. 2.1.3. Pode verse que $\alpha$ determina a massa efetiva $m^{* * *}$ de pares de férmions ligados (vestidos de bipolarons). A relação de dispersão é representado na Fig. 4.2. O parâmetro $\alpha$ pode ser recuperado de $m^{* * *}$. Uma estimativa dessa massa de dados experimentais em $\mathrm{La}_{2} \mathrm{CuO}_{4+y}$ podem ser encontrados em [21]: $m^{* * *} \in\left[m_{e}, 3 m_{e}\right]$. Este parâmetro não tem influência significativa em nosso estudo porque nós geralmente fixamos o momento total $K$. Apenas a forma precisa das Figs. 2.1.3 e 4.2 dependem de $\alpha$. Explicitamente pode mostrar-se ${ }^{3}$ que $m^{* * *} \simeq 2 m_{e}$ leva a $\alpha \simeq 1.35$, o qual é o nosso valor padrão.

\footnotetext{
${ }^{3}$ Por teoria de perturbações padrão para autovalores não degenerados, um calcula que, em $K=(0, \pi)$ e $(\pi, 0)$$$
\lambda(K+\eta, \varkappa \hat{v}((K+\eta))
$$$$
=\lambda(K, \varkappa)+\partial_{K_{x}}^{2} \lambda(K, \varkappa) \frac{\eta_{x}^{2}}{2}+\partial_{K_{y}}^{2} \lambda(K, \varkappa) \frac{\eta_{y}^{2}}{2}
$$$$
+\varkappa \partial_{\varkappa} \lambda(K, \varkappa) \partial_{K_{x}}^{2} \hat{v}(K) \frac{|\eta|^{2}}{2}+\mathcal{O}\left(|\eta|^{3}\right)
$$

for $\eta \in \mathbb{T}^{2},|\eta| \ll 1$. Portanto, a massa do o par de férmions ligados é igual a

$$
m^{* * *}=\frac{\mu_{\mathrm{ex}} \mu_{0}}{\mu_{0}+\mu_{\mathrm{ex}}} m_{e} \in m_{e} \min \left\{\mu_{\mathrm{ex}}, \mu_{0}\right\}[1 / 2,1],
$$

onde

$$
\begin{aligned}
\mu_{0} & \doteq \frac{\hbar^{2}}{m_{e} \mathbf{a}^{2}}\left(\begin{array}{cc}
\frac{1}{\partial_{K_{x}}^{2} \lambda(K, \varkappa)} & 0 \\
0 & \frac{1}{\partial_{K_{y}}^{2} \lambda(K, \varkappa)}
\end{array}\right), \\
\mu_{\mathrm{ex}} & \doteq-\frac{\hbar^{2}}{2 \alpha m_{e} \mathbf{a}^{2} \varkappa \partial_{\varkappa} \lambda(K, \varkappa)}\left(\begin{array}{cc}
1 & 0 \\
0 & 1
\end{array}\right)
\end{aligned}
$$

são, respectivamente, a massa intrínseca e induzida pela interação bipolaron-férmion em relação à massa do elétron $m_{e}$. Lembrando de que $\mathbf{a}=2.672 \AA$ é o espaçamento da rede. Estimativas numéricas em 


\section{Consequências fenomenológicas}

Os resultados a seguir correspondem ao setor de dois férmions e um bipolaron para parâmetros prototípicos tirados dos experimentos para o $\mathrm{La}_{2} \mathrm{CuO}_{4}$ ( $\left.\mathrm{LaSr} 214\right)$ perto do doping ótimo:

(a) No estado fundamental, demonstramos a existência de pares de férmions com quasimomentum total perto de $(\pi, 0)$ ou $(0, \pi)$, veja a Fig. 4.2. Os pares têm uma componente bipolarônica grande, isto é, são pares de férmions ligados vestidos, veja Fig. 4.3. Novamente pela Fig. 4.2, os pares de férmions vestidos se comportam como partículas maciças que têm energia mínima e estão em repouso sempre que seu quasimomentum é igual a $(\pi, 0)$ ou $(0, \pi)$.

$\overline{\text { parâmetros prototípicos para } K=(0, \pi)}$ dão

$$
\mu_{0} \simeq\left(\begin{array}{cc}
80 & 0 \\
0 & -52
\end{array}\right)
$$

Para $K=(\pi, 0), \mu_{0}$ é o mesmo, salvo uma permutação dos elementos diagonais. Portanto, para obter $m^{* * *} \simeq 2 m_{e}[21]$, basta ter $\mu_{\mathrm{ex}} \simeq 2$, que implica $\alpha \simeq 1.35$, o qual é o nosso valor padrão. ( $m^{* * *} \in\left[m_{e}, 3 m_{e}\right]$ leva a $\alpha \in[0.9,2.7]$.) 


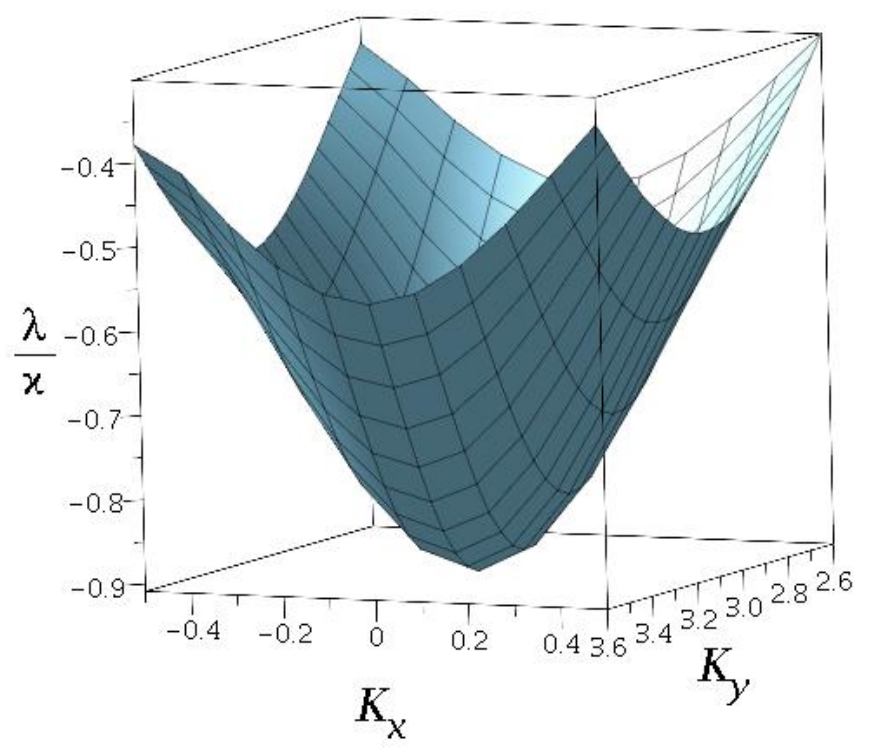

Figura 4.2: Energia de ligação $\lambda$ (em unidades de $\varkappa$ ) como uma função do quasimomentum total $K$ para parâmetros prototípicos.

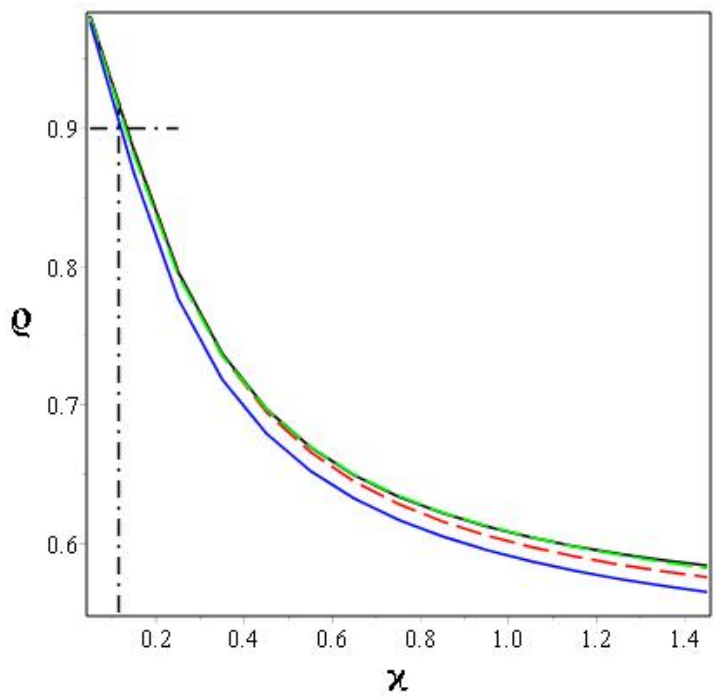

Figura 4.3: Depleção $\varrho$ como uma função de $\varkappa$ no total quasimomentum $K=(0, \pi)$ para magnitudes de repulsão local $U_{0}=1.461$ (azul), 5 (verde), 15 (preto), 50 (vermelho). As linhas vertical e horizontal de ponto-tracejado destacam a queda de $90 \%$ para os parâmetros prototípicos.

(b) A componente $p$-wave dos pares de férmions ligados com quasimomentum $(\pi, 0)$ ou 
$(0, \pi)$ é sempre zero. Ou seja, a simetria $C_{2}$ é não quebrada nessa caso, como para o $\mathrm{YBa}_{2} \mathrm{Cu}_{3} \mathrm{O}_{6+\delta}$ [23]. Em contraste, se a transformada de Fourier $\hat{v}$ da função de acoplamento $v$ é concentrada perto de $( \pm \pi / 2,0)$ e $(0, \pm \pi / 2)$, então o componente de $p$-wave dos pares domina. Veja abaixo Fig. 4.15. Como a componente $p$-wave nunca foi encontrado em cupratos supercondutores, esse fato fortalece nossa suposição de uma função $\hat{v}$ com seus máximos locais apenas em $(\pi, 0)$ e $(0, \pi)$.

(c) Na Fig. 4.4, obtemos $83.5 \%$ de componente $d$-wave e $16.5 \%$ de componente $s$ wave, de acordo com o que era deduzido de dados experimentais [24-26]. Veja também [11, Seção 6]. A existência de uma componente $d$-wave dominante e uma depleção forte ${ }^{4}$ (cerca de 90\%), como é observado em [22], estão vinculados. Veja linhas ponto-tracejadas nas Figs. 4.3 e 4.4. Em particular, a simetria $C_{4}$ é quebrada, como é observado para o $\mathrm{YBa}_{2} \mathrm{Cu}_{3} \mathrm{O}_{6+\delta}[23]$.
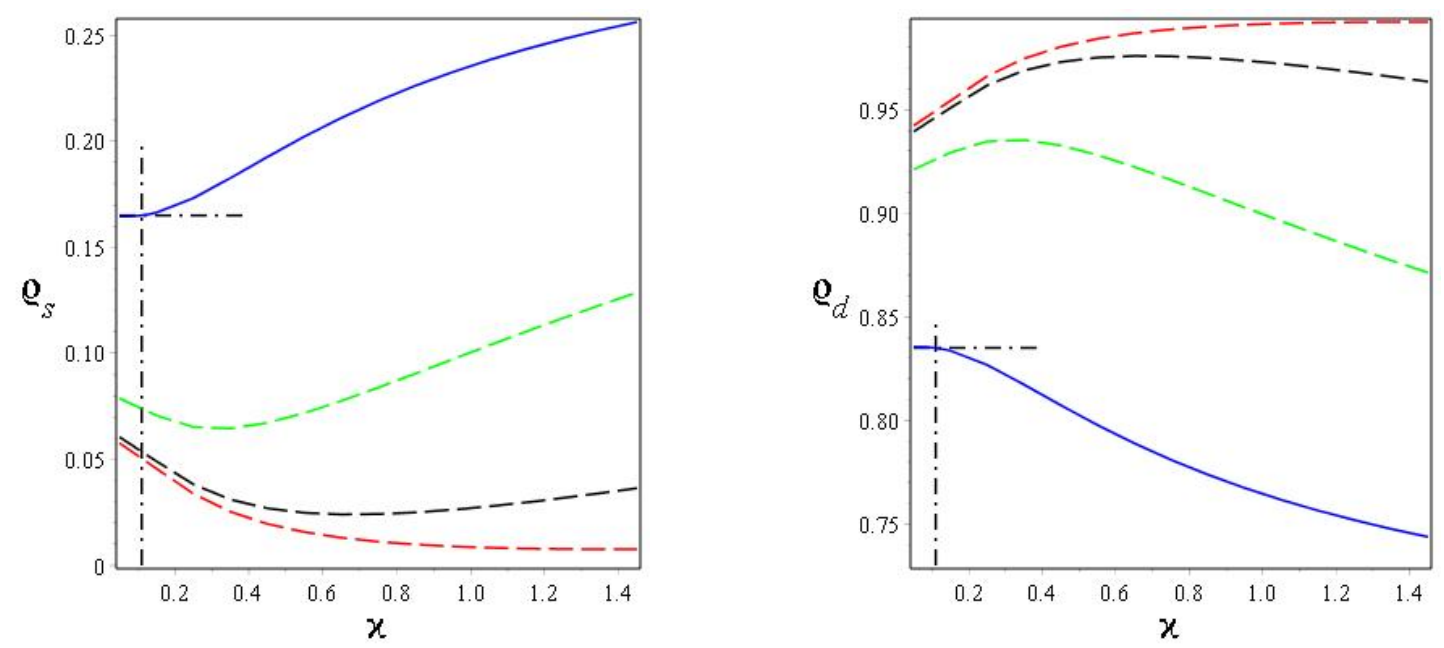

Figura 4.4: $s$ - e $d$-wave componentes (esquerda e direita, respectivamente) como funções de $\varkappa$ no quasimomentum total $K=(0, \pi)$ para magnitudes de repulsão local $U_{0}=1.461$ (azul), 5 (verde), 15 (preto), 50 (vermelho). As linhas verticais pontilhadas destacam o valor prototípico $\varkappa=0.11 \mathrm{eV}$.

(d) Calculamos no doping ideal um comprimento de coerência $\xi \simeq 8 \mathbf{a} \simeq 21 \AA$ (a é o espaçamento da rede), que é o mesmo obtido da teoria de Ginzburg-Landau

\footnotetext{
${ }^{4}$ Isto significa que a componente fermiônica dos pares de fermiões ligados torna-se muito pequeno em comparação com a componente bipolarônica.
} 
para este material. Veja Fig. 4.5 (em unidades da rede) e [13, Tabela 9.1]. Note que a compreensão da pequenez do tamanho dos pares de Cooper em cupratos supercondutores é uma questão importante na teoria microscópica de supercondutividade de alta temperatura.

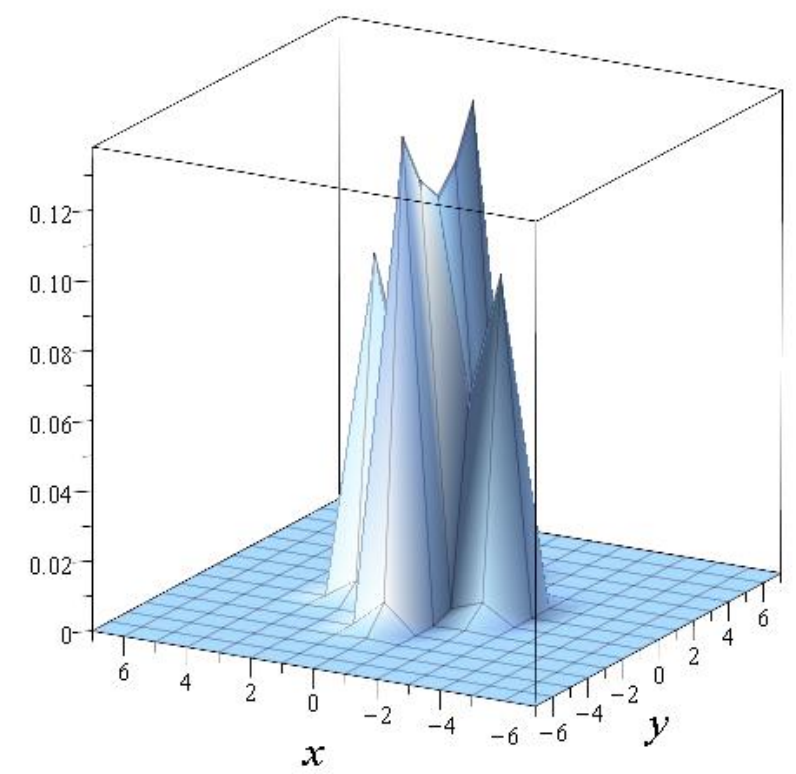

Figura 4.5: Densidade normalizada $\left|\check{\psi}_{(0, \pi)}\right|^{2}$ do par de férmions ligados como função da posição relativa em $K=(0, \pi)$ para parâmetros prototípicos.

(e) Nossa teoria prevê que os pares $d$-wave dominam o regime do pseudogap, ou seja, a temperaturas muito superiores às temperatura de transição supercondutora de 39K para o dopagem ótimo de o $\mathrm{La}_{2} \mathrm{CuO}_{4}$ [13, Fig. 5.1]. Veja as linhas pretas pontotracejadas da Fig. 4.6. É predito que a temperatura do pseudogap dependa da força da repulsão coulombiana inter-site (Fig. 4.11), mas não muito da local (Fig. 4.6). No entanto, em parâmetros prototípicos, um blindagem mais forte da interação de Coulomb não aumentaria significativamente a temperatura de pseudogap (ver Fig. 4.11). Para dopagem ótimo de $\mathrm{La}_{2} \mathrm{CuO}_{4}$, a temperatura do pseudogap é 400K [27, Fig. 26]. 


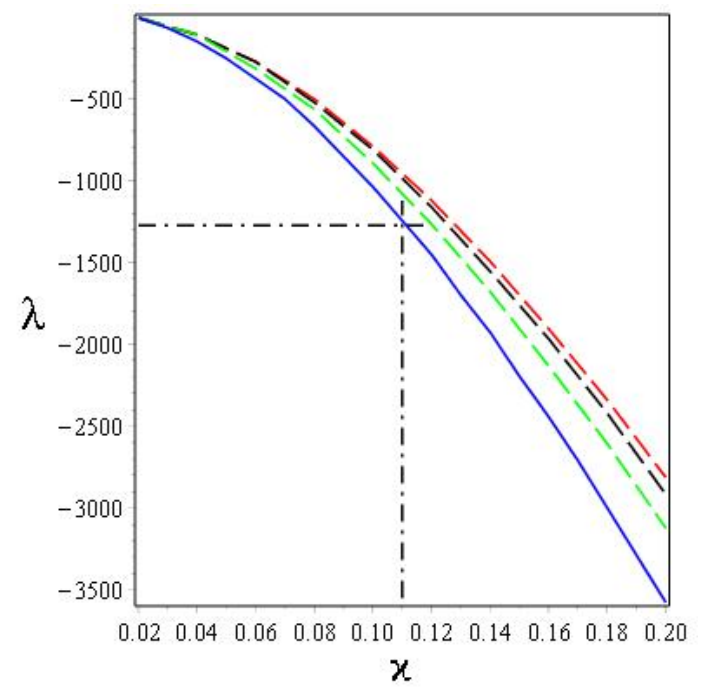

Figura 4.6: Energia de ligação $\lambda$ (em Kelvin) como função de $\varkappa$ em total quasimomentum $K=(0, \pi)$ para magnitude de repulsão local $U_{0}=1.461$ (azul), 5 (verde), 15 (preto), 50 (vermelho). As linas vertical e horizontal pontilhadas destacam o valor prototípico $\varkappa=0.11 \mathrm{eV}$.

(f) Interação bipolaron-férmion local implica pares de elétrons ligados com componente puramente $s$-wave. Uma supressão considerável da componente $d$-wave dos pares de férmions ligados também são encontrados na ausência da repulsão de Coulomb. Veja Fig. 4.12 (à direita) para mais detalhes. Consequentemente, tanto a repulsão eletrônica e os bipolarons inter locais são necessários para ter uma componente $d$-wave dominante.

(g) Não são necessárias repulsões de Coulomb fortes para obter a depleção forte (fermiônica), medida em [22]. Veja Fig. 4.12 (à esquerda). A forte depleção está relacionada, em vez disso, ao masa grande dos bipolarons, como vemos na Fig. 4.9. Além disso, a temperatura grande de pseudogap [27, Fig. 26] não é compatível com bipolarons leves. Veja a Fig. 4.10. A grande massa dos bipolarons estimados em [20] é, no entanto, desnecessário para que nossa teoria microscópica funcione: um bipolaron 10 vezes mais leve produziria a mesma fenomenologia, embora ainda haja de ser significativamente mais pesado do que os buracos.

(h) A massa calculada em [21] para os portadores de carga devem ser vistos como uma 
massa estimada para os pares de fermiões em vez de bipolarons. Nosso modelo prediz que uma massa efetiva grandem*** de pares férmions ligados não é compatível com uma depleção perto de 90\%, medida em [22]. Mais precisamente, em tais depleções, nossa teoria microscópica implica que $m^{* * *} \leq 80 m_{e}$, onde $m_{e}$ é a massa do elétron. $\mathrm{Na}$ verdade, o valor exato de $m^{* * *}$ depende fortemente da função de acoplamento $\hat{v}$ perto do seu máximo (em pontos de van Hove neste caso). Usando a estimativa $m^{* * *} \in\left[m_{e}, 3 m_{e}\right]$ de [21], concluímos que $\hat{v}$ é apenas fracamente concentrado em torno dos pontos $\{( \pm \pi, 0),(0, \pm \pi)\}$, veja a Fig. 2.1.3. Isto é coerente com o relação da dispersão de fônons exibido em [28, Fig. 2].

(i) Finalmente, o regime do núcleo duro, ou seja, $U_{0} \gg 1$, captura bem as propriedades físicas dos pares de férmions ligados vestidos. Veja as Figs. 4.3 (depleção), 4.4 (componentes $s$ - e $d$-wave) e 4.6 (energia de ligação). A estrutura das correlações dos pares também está bem descrita neste regime: em comparação com a Fig. 4.5, apenas a densidade na origem é afetado, na medida em que é fortemente suprimido, o que está de acordo com o maior componente $d$-wave vista na Fig. 4.4, para $U_{0} \gg 1$. Em contraste, nem o pequeno hopping $(\epsilon)$ nem o grande (simultaneamente local e inter-site) regimes de repulsão podem reproduzir corretamente a fenomenologia de pares de fermiões ligados em parâmetros prototípicos para dopados $\mathrm{La}_{2} \mathrm{CuO}_{4}$. Veja as Figs. 4.11, 4.13 e ref fig-new-11. Isso demonstra que o aparamento em cupratos é um fenômeno essencialmente quântico.

\section{Simetrias na Função de Onda dos Pares}

Como foi feito no capitulo anterior, estudamos aqui o autovetor $\left(\left|\psi_{K}\right\rangle, 1\right)$ de $A(K)$ associado a um autovalor estritamente negativo $\lambda \equiv \lambda(K, \varkappa \hat{v}(K))<0$ para aqueles momentos totais $K$ que maximizam a função de acoplamento $|\hat{v}(K)|$, normalmente $(\pi, 0)$ e $(0, \pi)$. O parâmetro que quantifica a força da interação de acoplamento $\varkappa$ é escolhida para obter $90 \%$ de depleção em $(\pi, 0)$ e $(0, \pi)$. A depleção em momento total $K$ é definido 
pela relação

$$
\varrho \doteq \frac{100}{\left(\left\|\psi_{K}\right\|_{2}^{2}+1\right)} \%
$$

Usando parâmetros prototípicos fornecidos na seção anterior, a Fig. 4.3 (curva azul) representa essa relação como uma função de $\varkappa$ para $K=(0, \pi)$. Isso leva a $\varkappa \simeq 0.11 \mathrm{eV}$ quando $\varrho \simeq 90 \%$.

A componente dominante $\left(s^{-}, d^{-}\right.$ou $p$-wave $)$ de $\left|\psi_{K}\right\rangle$ determina a simetria de emparelhamento do par fermiônico ligado com quasimomentum total $K$. Para $K \in$ $\{(0, \pi),(0, \pi)\}$, a componente $p$-wave é zero ou $P_{p}\left|\psi_{K}\right\rangle=0$. Em geral ${ }^{5}, \psi_{K}$ é uma mistura não trivial de componentes $s$ - e $d$-wave. Usando os parâmetros prototípicos, derivamos as componentes $s$ e $d$-wave do par do férmions ligados como uma função de $\varkappa$ at $K=(0, \pi)$. Veja a Fig. 4.4 (curvas azuis).

Para $\varkappa \simeq 0.11 \mathrm{eV}$, ou seja, com $90 \%$ de depleção, a componente $d$-wave é quase maximizada para os parâmetros prototípicos. Nesse caso, temos

$$
\varrho_{s} \doteq \frac{\left|P_{s} \psi_{(0, \pi)}\right|_{2}^{2}}{\left|\psi_{(0, \pi)}\right|_{2}^{2}} \times 100 \% \simeq 16.5 \%
$$

componente $s$-wave, que é relativamente próximo da estimativa $(20 \%$ - 25\%) deduzida fenomenologicamente dos dados experimentais [24-26]. Veja também [11, Seção 6].

A densidade (normalizada) $\left|\breve{\psi}_{(0, \pi)}\right|^{2}$ do par de férmions ligados no espaço de posição (coordenada relativa) está representado na Fig. 4.5. É anisotrópica por causa da massa negativa dos férmions livres em apenas um dos eixos no ponto de van Hove $(0, \pi)$. O par fermiônico correspondente ao outro ponto de van Hove $(\pi, 0)$ tem função de onda $\left|\psi_{(\pi, 0)}\right\rangle=R_{\perp}\left|\psi_{(0, \pi)}\right\rangle$. Portanto, até rotações de $\pi / 2$, tem a mesma estrutura espacial que $\left|\psi_{(0, \pi)}\right\rangle$. Ambas as funções estão fortemente concentradas em uma região de maior diâmetro $\xi_{\max } \simeq 8$ (em unidades de rede), ou seja, $\xi_{\max } \simeq 8 \mathbf{a}=21.376 \AA$. Isso é quase o mesmo que o comprimento de coerência $\xi=21 \AA$ do $\mathrm{La}_{1.8} \mathrm{Sr}_{0.2} \mathrm{CuO}_{4}$, o qual é calculado a partir de dados experimentais através da teoria de Ginzburg-Landau. Veja [13, Tabela 9.1] e referências nele contidas. Pela Fig. 4.5, o menor diâmetro é igual a $\xi_{\min } \simeq 6$ (em

\footnotetext{
${ }^{5}$ Para $\varkappa=1, r=0, K \in\{(0, \pi),(0, \pi)\}$, a componente $s$-wave apenas desaparece no limite $\epsilon \rightarrow 0$, $U \rightarrow \infty$, por [29, Theorem 4].
} 
unidades de rede), isto é, $\xi_{\min } \simeq 6 \mathbf{a}=16.032 \AA$. Observe que [21] dá a estimativa $\xi \gtrsim 16 \AA$ para o diâmetro do par ligado, que é derivado de dados experimentais para $\mathrm{La}_{2} \mathrm{CuO}_{4+y}$ combinados com um simples modelo hidrogênico (e não da teoria de Ginzburg-Landau).

Os cálculos numéricos mostram que $\xi_{\min }$ e $\xi_{\max }$ não dependem muito da escolha do alcance $r$ da repulsão: $\xi_{\max } \simeq 8 \mathbf{a}$ e $\xi_{\min } \simeq 6 \mathbf{a}$ para $r=0,1,1,5,2$. Os efeitos do parâmetro $r$ em na depleção, a magnitude da componente $d$-wave e a energia de ligação, como é mostrado nas Figs. 4.7 e 4.11 (à esquerda), também são mínimos. No entanto, as alterações em $r$ modificam a forma de $\left|\check{\psi}_{(0, \pi)}\right|^{2}$. Por exemplo, para $r=0$ o valor máximo de $\left|\breve{\psi}_{(0, \pi)}\right|^{2}$ é atingido a distância 1 (em unidades de rede) da origem, enquanto que, para $r=1,1,5,2$, o máximo é atingido a distância 2. Compare a Fig. 4.5 para $r=2 \mathrm{com}$ a Fig. 4.8 para $r=0$.
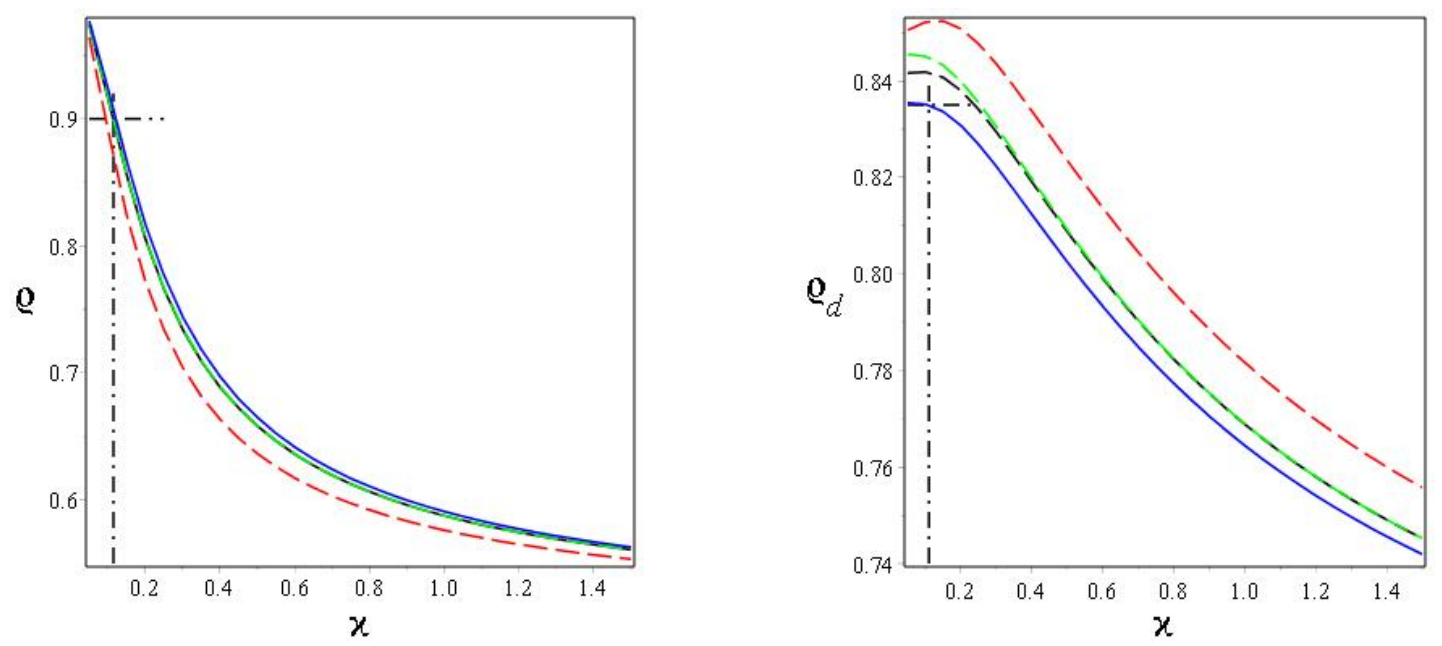

Figura 4.7: Depleção (esquerda) e componente $d$-wave (direta) como função de $\varkappa$ em total quasimomentum $K=(0, \pi)$ para $r=0$ (vermelho), 1 (preto), 1.5 (verde) e 2 (azul). 


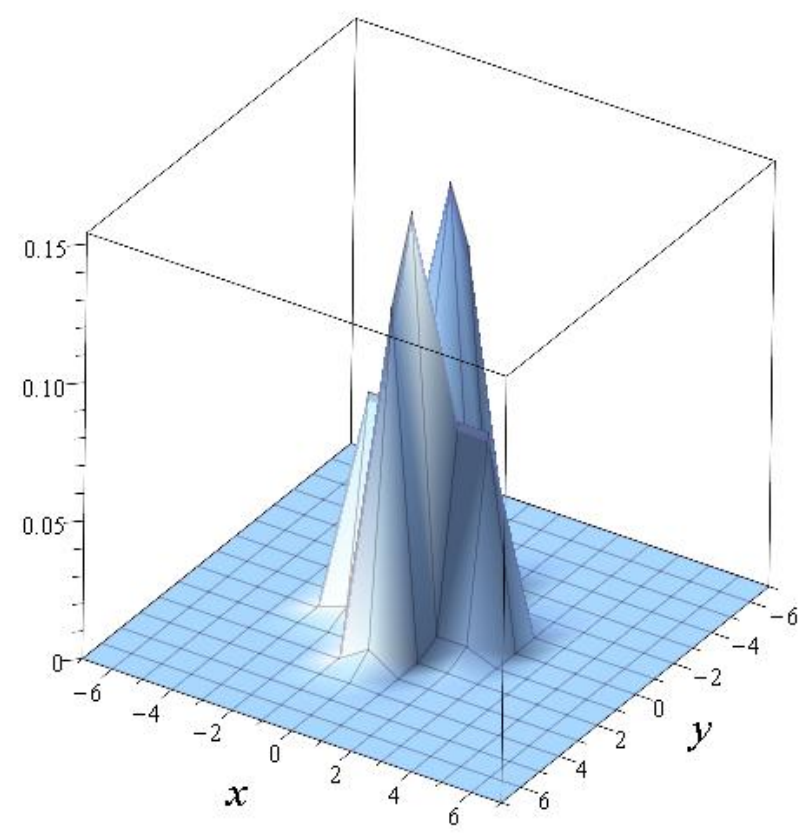

Figura 4.8: Densidade normalizada $\left|\breve{\psi}_{(0, \pi)}\right|^{2}$ de o par fermiônico ligado como função da coordenada relativa em total quasimomentum $K=(0, \pi)$ para repulsão local $(r=0)$.

Como foi explicado anteriormente, a massa efetiva $m^{* *}$ dos bipolarons é controversa. Seu valor prototípico neste estudo é baseado em [20]. Isso corresponde a $h_{b} \simeq 0.00575$, mas nas Figs. 4.9 e 4.10 também consideramos valores maiores deste parâmetro, até o caso $h_{b}=1$. Verificou-se que uma massa efetiva $m^{* *}$ até 10 vezes menor do que a $\left(\simeq 695 m_{e}\right)$ estimado em $[20]$ produziria o par fermiônico ligado com quase exatamente as mesmas propriedades físicas que aqueles com valores padrões de parâmetros. A depleção (fermiônica) nunca pode exceder $75 \%$ quando $m^{* *} \leq 8 m_{e}$ e $\varkappa \leq 0.2 \mathrm{eV}$, veja Figs. 4.9 e 4.10. Além disso, a energia de ligação dos pares de férmions ligados são fortemente reduzidos para $m^{* *} \leq 8 m_{e}$, em comparação com a situação prototípica. Veja a Fig.4.10. Lembrando, no entanto, de que a pequena massa calculada em [21] não é necessariamente contraditória com o valor grande da massa determinada em [20] uma vez que, em nosso modelo, eles se referem a massas de dois quase-partículas distintas: bipolarons em [20] e pares de férmions ligados em [21]. 

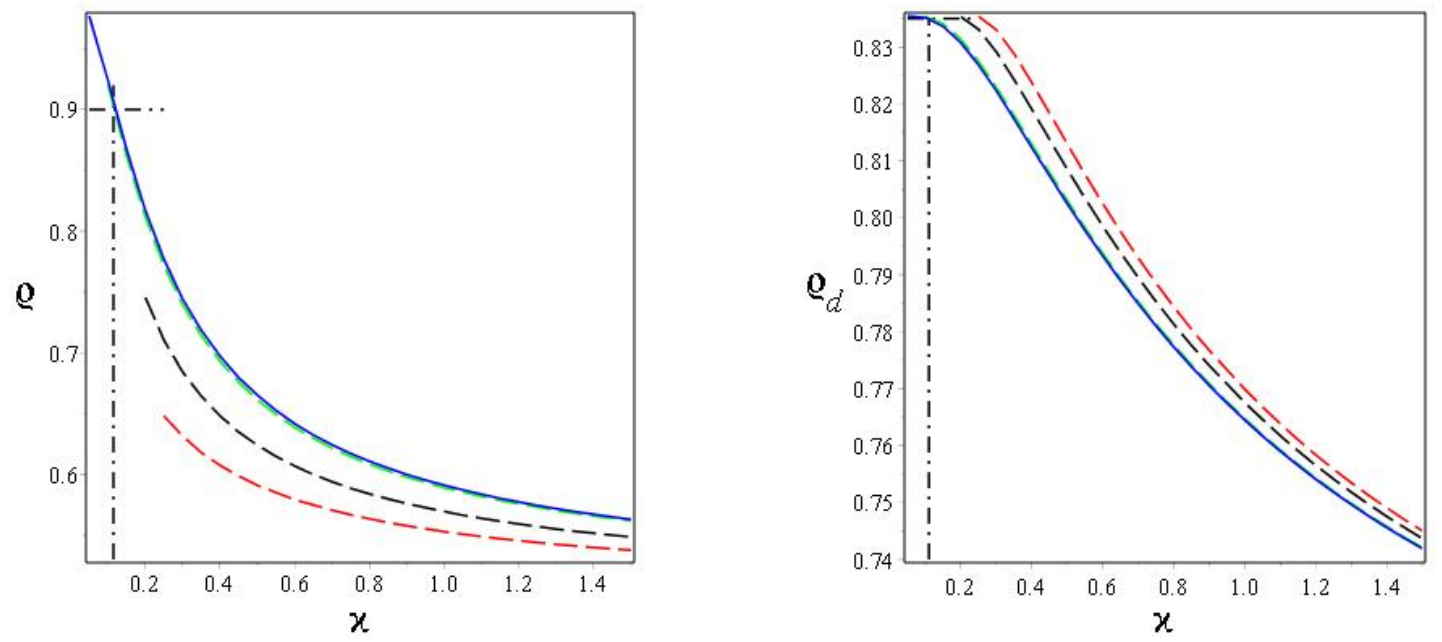

Figura 4.9: Depleção (esquerda) e componente $d$-wave (direta) como função de $\varkappa$ em total quasimomentum $K=(0, \pi)$ para $h_{b}=0.00575$ (azul), 0.0575 (verde), 0.575 (preto), 1 (vermelho).

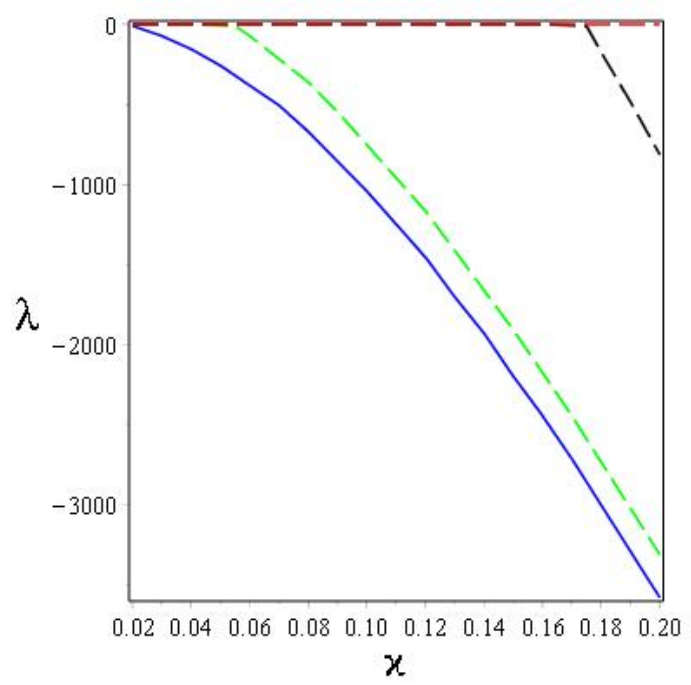

Figura 4.10: Energia de ligação $\lambda$ (em Kelvin) como função de $\varkappa$ em total quasimomentum $K=(0, \pi)$ para $h_{b}=0.00575$ (azul), 0.0575 (verde), 0.575 (preto), 1 (vermelho). 

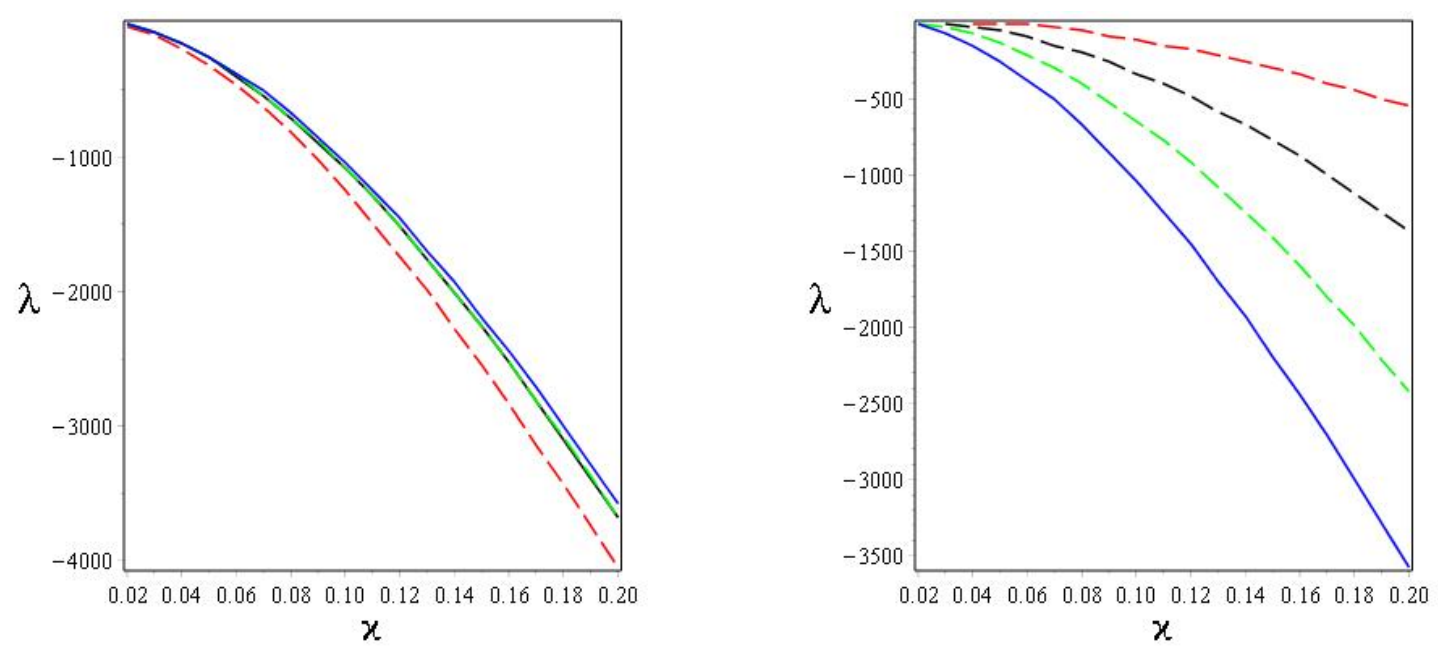

Figura 4.11: Energia de ligação $\lambda$ (em Kelvin) como função de $\varkappa$ em total quasimomentum $K=(0, \pi)$. Na figura da esquerda, $r=0$ (azul), 1 (verde), 1.5 (preto), 2 (vermelho). Na figura da direta, $U=1.461$ (azul), 5 (verde), 15 (preto), 50 (vermelho). As linhas pontilhadas vertical e horizontal ressaltam o parâmetro prototípico $\varkappa=0.11 \mathrm{eV}$.

A natureza inter-site dos bipolarons e a presença da repulsão de Coulomb forte é essencial para obter-se uma grande $(i 3)$ razão entre a componente $d$-wave e $s$-wave. Isso é visto na Fig. 4.12, onde as linhas azul e vermelha representam os casos $U_{0}=U=0$ (sem repulsão de Coulomb) e $c_{x}=2 a_{x, \uparrow} a_{x, \downarrow}$ (bipolarons locais, não inter-site como em (4.4)), respectivamente. Além disso, a Fig. 4.13 demonstra que os comportamentos da depleção e o pareamento não são bem reproduzidos no regime de hopping $(\epsilon)$ pequeno. Em particular, a simetria dos pares fermiônicos ligados depende não apenas da competição entre a interação bipolaron-fermion e a repulsão de Coulomb, mas também da energia cinética. Por exemplo, no limite de hopping pequeno (massa efetiva de férmions grande) a simetria da função de onda associada aos pares de férmions é puramente $d$-wave com uma depleção que não excede $75 \%$. 

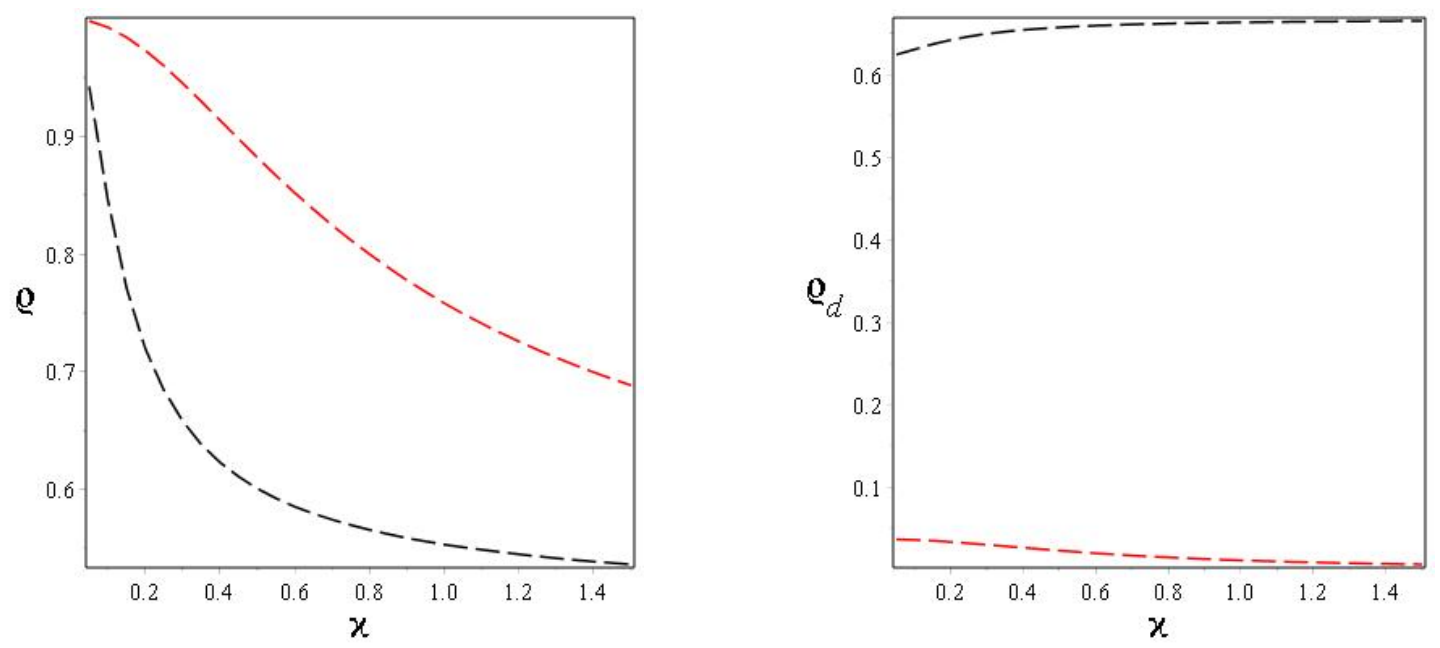

Figura 4.12: Depleção (esquerda) e componente $d$-wave (direta) como função de $\varkappa$ em total quasimomentum $K=(0, \pi)$ para $U=0$ (não repulsão, preto) e bipolarons locais (vermelho).

Por outro lado, como já foi discutido, o regime do núcleo duro $U_{0} \gg 1$ capta bastante bem as propriedades físicas dos pares de férmions ligados, pelas figs. 4.3 (depleção), 4.4 (componentes $s$ - e $d$-wave) e 4.6 (energia de ligação). Da Fig. 4.14 note que o regime de repulsão forte $U_{0}=U \gg \varkappa$ produz uma componente $d$-wave quase pura, mas não é compatível com o 90\% da depleção. Além disso, a energia de ligação dos pares de fermiões ligados diminui fortemente para grandes $U_{0}=U \gg \varkappa$, pela Fig. 4.11. 

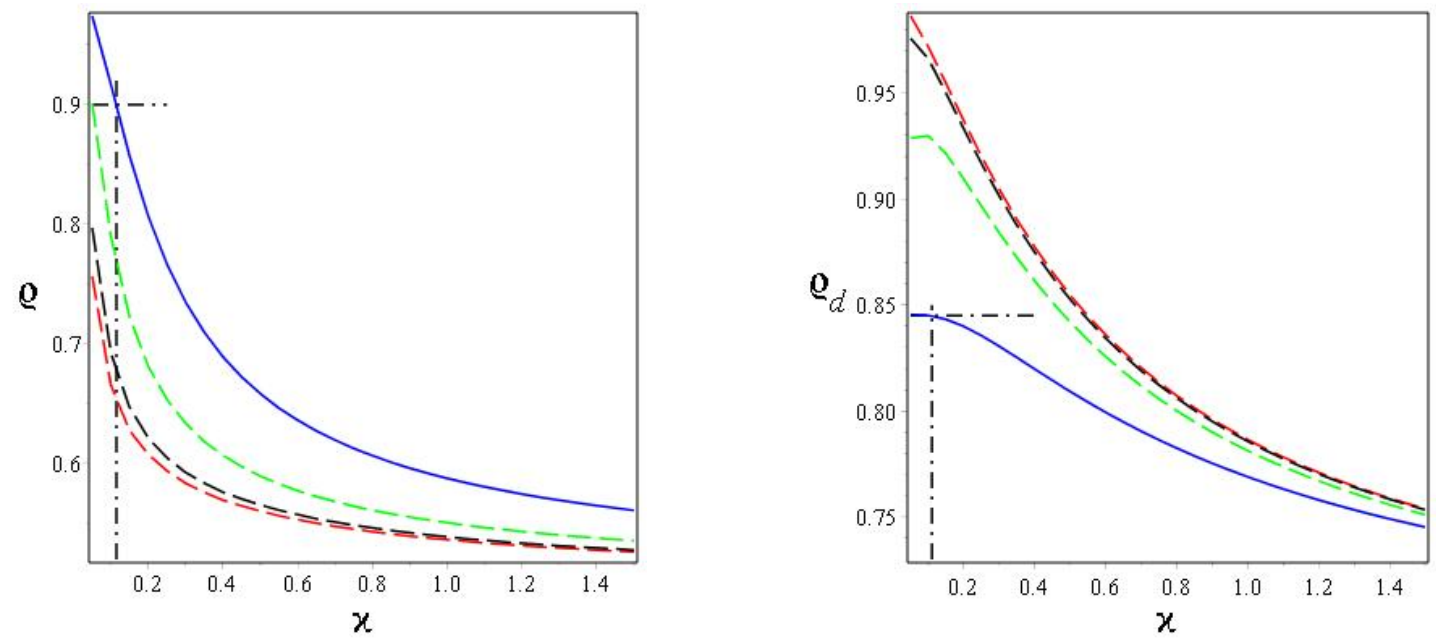

Figura 4.13: Depleção (esquerda) e componente $d$-wave (direta) como função de $\varkappa$ em total quasimomentum $K=(0, \pi)$ para amplitudes de hopping $\epsilon=0.266$ (azul), $0.266 \times 25 \%$ (verde), $0.266 \times 5 \%$ (preto), $0.266 \times 1 \%$ (vermelho). As linhas pontilhadas vertical e horizontal ressaltam os parâmetros prototípicos.
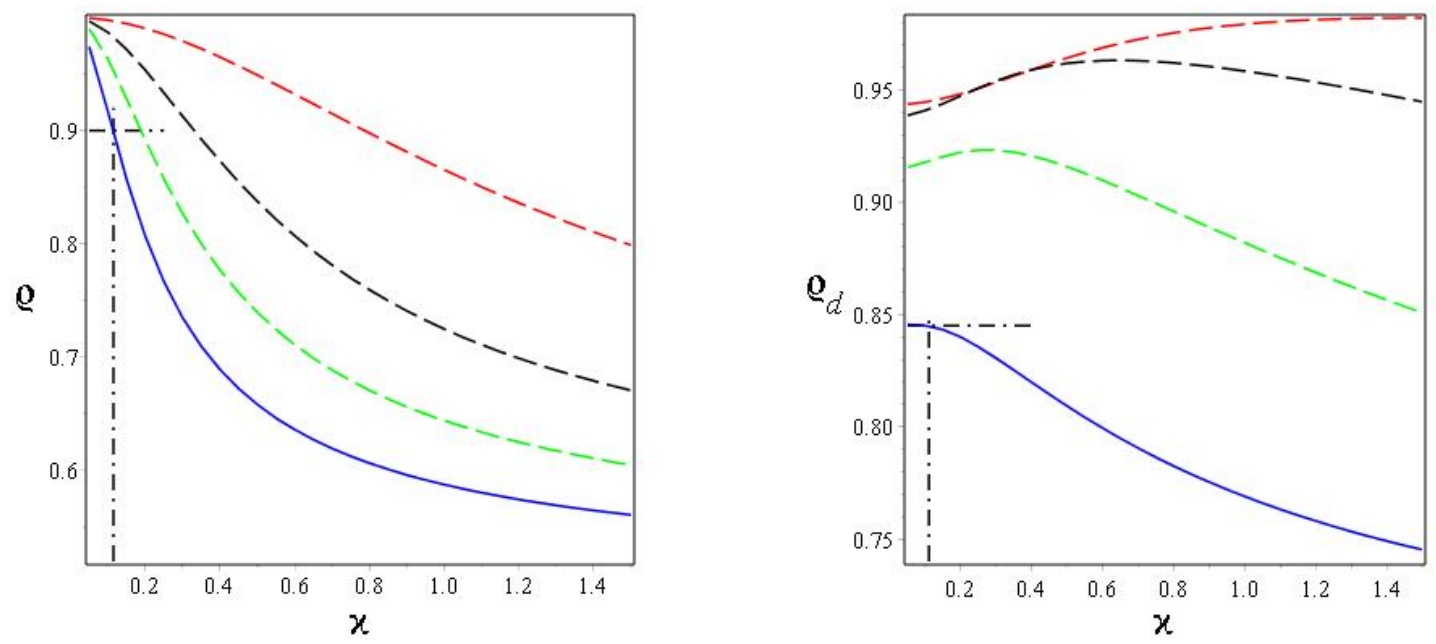

Figura 4.14: Depleção (esquerda) e componente $d$-wave (direta) como função de $\varkappa$ em total quasimomentum $K=(0, \pi)$ para $U=1.461$ (azul), 5 (verde), 15 (preto), 50 (vermelho).

Concluímos estudando o caso em que $\hat{v}(K)$ está concentrado em $K=(0, \pm \pi / 2)$ e $( \pm \pi / 2,0)$, em vez de $(\pi, 0)$ e $(0, \pi)$. Nesse caso como foi explicado anteriormente, aparece uma componente $p$-wave da função de onda fermiônica. Em $K=(0, \pi / 2)$ (os outros casos são equivalentes), as componentes $s^{-}, d$ - e $p$-wave de $\left|\psi_{(0, \pi / 2)}\right\rangle$ em parâmetros prototípicos 
são representadas na Fig. 4.15 como uma função de $\varkappa$. Nós observamos que a formação de pares $p$-wave é sempre favorecida em $90 \%$ de depleção.
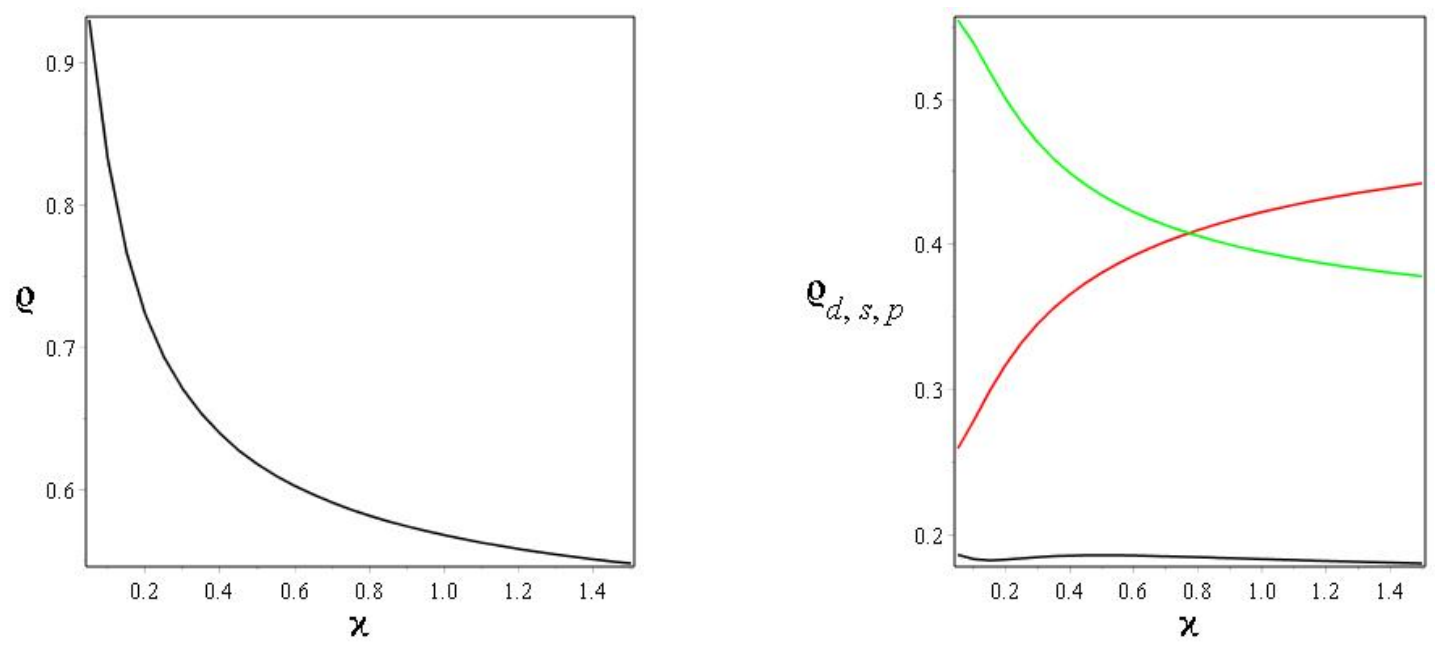

Figura 4.15: Depleção (esquerda) e componentes $s-, d$-, $p$-wave (direta) como função de $\varkappa$ em total quasimomentum $K=(0, \pi / 2)$ para parâmetros prototípicos. As componentes $s^{-}, d$ - e $p$-wave são representadas em vermelho, preto e verde respetivamente.

Isso demonstra, como foi discutido também no capitulo anterior, que a existência de pares $d$-wave em cupratos supercondutores está diretamente relacionado ao fato de que $\hat{v}(K)$ é concentrado próximo dos pontos $(K=(0, \pi),(\pi, 0))$. Longe desses pontos, parecem aparecer pares $p$-wave e são dominantes em $K=(0, \pm \pi / 2$ e $( \pm \pi / 2,0)$. Não há pares $p$-wave observados em cupratos supercondutores e nosso estudo sugere que as anomalias nos pontos onde a interação bipolaron-elétron domina no espaço de momentum são, portanto, parte do mecanismo físico que leva à formação deste tipo de pares no outros materiais.

Os pares fermiônicos $p$-wave foram observados em outros superconductores com alta temperatura de transição (não cupratos). De fato, o rutenato de estrôncio $\mathrm{Sr}_{2} \mathrm{RuO}_{4}$ é a baixa temperatura um supercondutor dentro de camadas de $\mathrm{RuO}_{2}$ e apresenta simetria $p$-wave, veja, por exemplo, [30, Tabela I]. No entanto, nosso estudo não se aplica a esse tipo de material porque consideramos apenas aqui pares de férmions que formam singletos de spin; os pares supercondutores em $\mathrm{Sr}_{2} \mathrm{RuO}_{4}$ são tripletos de spin, semelhantes aos do superfluido quântico ${ }^{3} \mathrm{He}$. 


\section{Capítulo 5}

\section{Modelo Fermiônico Efetivo e Ondas de Densidade}

Para o caso de dois elétrons e um bipolaron mostramos que no modelo proposto existe um estado ligado de elétrons resultante da interação mediada pelo bipolaron. No limite em que a repulsão coulombiana é forte mostramos também que a simetria da função de onda fermiônica é do tipo $d$-wave tal como é observado experimentalmente para alguns supercondutores de alta temperatura crítica, como os cupratos. O limite tomado antes onde a energia cinética dos elétrons e o bipolaron é desprezível com respeito as interações entre os elétrons é conhecido como limite de acoplamento forte. Nesta região á analise do problema é mais simples e esta justificado pelo fato experimental da energia de interação entre partículas ser cerca de oito vezes superior a energia cinética das mesmas.

O analise do problema dos três corpos é relevante para o estudo da interação entre dois elétrons, mas na realidade a supercondução é um fenômeno coletivo e, portanto, o problema a muitos corpos deverá ser abordado. Os métodos usados para o estudo do problema dos três corpos não são viáveis para a analise do problema de os infinitos corpos, mas a primeira aproximação natural é tomar o limite de acoplamento forte tal como foi feito para o caso do três corpos. Além de tomar o limite de acoplamento forte, é possível considerar um Hamiltoniano efetivo que desacopla o setor fermiônico do setor bosônico e trata o problema como um caso sem interação entre bósons e férmions. 
O objetivo da analise do problema de infinitos corpos é encontrar os estados de equilíbrio, que são agora funcionais normalizados positivos na álgebra CAR associada aos férmions na rede. Mostraremos a condição para que na situação de equilíbrio se apresentem ondas de densidade, tal como é observado em cupratos supercondutores.

\section{Hamiltoniano Fermiônico Efetivo}

Por simplicidade consideraremos só o caso com repulsão local, ou seja

$$
H_{f}^{(0)}=\epsilon\left(-\frac{1}{2} \sum_{s \in\{\uparrow, \downarrow\}, x, y \in \mathbb{Z}^{2},|x-y|=1} a_{x, s}^{\dagger} a_{y, s}+2 \sum_{s \in\{\uparrow, \downarrow\}, x \in \mathbb{Z}^{2}} a_{x, s}^{\dagger} a_{x, s}\right)+\sum_{x \in \mathbb{Z}^{2}} U_{0} n_{x, \uparrow} n_{x, \downarrow} .
$$

Agora consideramos um termo de interação efetivo que atua sobre o espaço fermiônico e que deve simular a interação elétron-bipolaron no limite de baixa energia cinética $(\epsilon \rightarrow 0)$. Tal interação efetiva é considerada similar ao modelo BCS, então propomos

$$
W^{\prime}=-\sum_{x, y \in \mathbb{Z}^{2}} w(x-y) c_{x}^{\dagger} c_{y}
$$

onde

$$
c_{x} \doteq \sum_{z \in \mathbb{Z}^{2},|z| \leq 1} \mathrm{e}^{-\kappa|z|} a_{x+z, \uparrow} a_{x, \downarrow}
$$

que corresponde ao caso $\gamma \kappa^{-1}=1$ em (2.4).

Definimos então o Hamiltoniano efetivo $\widetilde{H}_{f}$

$$
\widetilde{H}_{f} \doteq H_{f}^{(0)}+W^{\prime}
$$

Agora só falta determinar a função $w(x-y)$ que simule corretamente a dinâmica do problema original no limite $\epsilon \rightarrow 0$ e repulsão local forte $\left(U_{0} / \varkappa>>1\right)$. Com o fim de encontrar esta função podemos usar os resultados obtidos para o caso do problema dos três corpos. Primeiro calcularemos a fibra $\widetilde{A}(K)$ do Hamiltoniano $\widetilde{H}_{f}$, e iremos impor a condição de que a autofunção (2.31) (no limite "strong coupling" $\epsilon \rightarrow 0, U_{0} / \varkappa>>1$ ) seja 
autofunção também de $\widetilde{A}(K)$ com a mesma energia $E_{0}$ do estado fundamental de nosso Hamiltoniano original.

Para o cálculo da fibra $\widetilde{A}(K)$ seguimos os mesmos passos feitos anteriormente para o cálculo da fibra $A(K)$. Calculando para o único termo diferente $W^{\prime}$, no espaço dos dois elétrons, temos

$$
\begin{gathered}
{\left[\Psi_{\uparrow \downarrow}^{\prime}(K)\right]\left(k_{\uparrow \downarrow}\right)=\mathfrak{U}_{f}^{\dagger}\left(\sum_{x, y \in \mathbb{Z}^{2}} w(x-y) c_{x}^{\dagger} c_{y}\right) \phi_{f}\left(x_{\uparrow}, x_{\downarrow}\right)} \\
c_{y} \phi_{f}\left(x_{\uparrow}, x_{\downarrow}\right)=\sum_{z \in \mathbb{Z}^{2},|z| \leq 1} \mathrm{e}^{-\kappa|z|} a_{y+z, \uparrow} a_{y, \downarrow} \phi_{f}\left(x_{\uparrow}, x_{\downarrow}\right)=\sum_{z \in \mathbb{Z}^{2},|z| \leq 1} \mathrm{e}^{-\kappa|z|} \delta_{y+z, x_{\uparrow}} \delta_{y, x_{\downarrow}}|0\rangle \\
\sum_{x, y \in \mathbb{Z}^{2}} w(x-y) c_{x}^{\dagger} c_{y} \phi_{f}\left(x_{\uparrow}, x_{\downarrow}\right) \\
=\sum_{x, y \in \mathbb{Z}^{2}} w(x-y) \sum_{z, z^{\prime} \in \mathbb{Z}^{2},|z| \leq 1,\left|z^{\prime}\right| \leq 1} \mathrm{e}^{-\kappa|z|} \mathrm{e}^{-\kappa\left|z^{\prime}\right|} \delta_{y+z, x_{\uparrow}} \delta_{y, x_{\downarrow}} \phi_{f}\left(x+z^{\prime}, x\right) .
\end{gathered}
$$

Aplicando $\mathfrak{U}_{f}^{\dagger}$

$$
\sum_{x_{\uparrow}, x_{\uparrow \downarrow} \in \mathbb{Z}^{2}} \mathrm{e}^{-i K \cdot x_{\uparrow}} \mathrm{e}^{-i k_{\uparrow \downarrow} \cdot x_{\uparrow \downarrow}} \sum_{x, y \in \mathbb{Z}^{2}} w(x-y) c_{x}^{\dagger} c_{y} \phi_{f}\left(x_{\uparrow}, x_{\downarrow}\right)
$$

temos

$\sum_{x_{\uparrow}, x_{\uparrow \downarrow}, x, y \in \mathbb{Z}^{2}} \mathrm{e}^{-i K \cdot x_{\uparrow}} \mathrm{e}^{-i k_{\uparrow \downarrow} \downarrow x_{\uparrow \downarrow}} w(x-y) \sum_{z, z^{\prime} \in \mathbb{Z}^{2},|z| \leq 1,\left|z^{\prime}\right| \leq 1} \mathrm{e}^{-\kappa|z|} \mathrm{e}^{-\kappa\left|z^{\prime}\right|} \delta_{y+z, x_{\uparrow}} \delta_{y, x_{\downarrow}} \phi_{f}\left(x+z^{\prime}, x\right)$.

Somando sobre $x_{\uparrow}$ e $x_{\downarrow}$

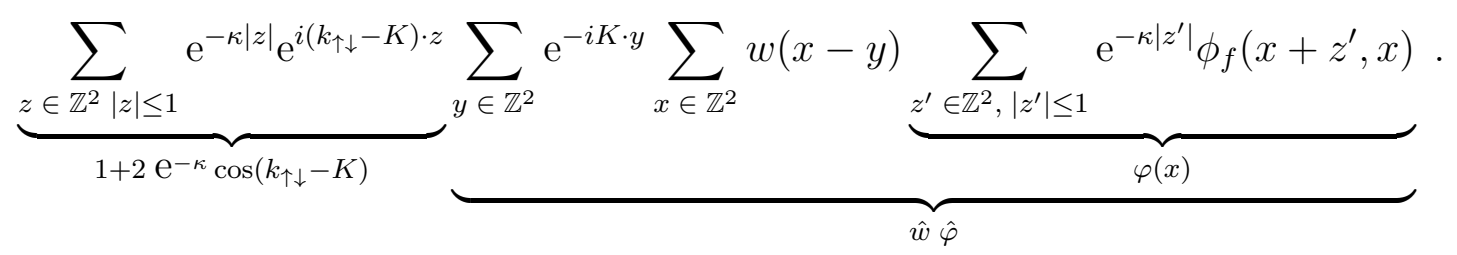


Seguindo os mesmos passos que no (2.16) temos

$$
\hat{\varphi}=\left\langle 1+2 \mathrm{e}^{-\kappa} \cos \left(k_{\uparrow \downarrow}-K\right),\left[\Psi_{\uparrow \downarrow}(K)\right]\left(k_{\uparrow \downarrow}\right)\right\rangle
$$

pelo que

$$
\left[\Psi_{\uparrow \downarrow}^{\prime}(K)\right]\left(k_{\uparrow \downarrow}\right)=\hat{w}\left(1+4 \mathrm{e}^{-2 \kappa}\right) P_{s+d}\left[\Psi_{\uparrow \downarrow}(K)\right]\left(k_{\uparrow \downarrow}\right)
$$

onde o projetor ortogonal $P_{s+d}$ é definido assim

$$
P_{s+d}=\frac{1}{1+4 \mathrm{e}^{-2 \kappa}}\left|1+2 \mathrm{e}^{-\kappa} \cos \left(k_{\uparrow \downarrow}-K\right)\right\rangle\left\langle 1+2 \mathrm{e}^{-\kappa} \cos \left(k_{\uparrow \downarrow}-K\right)\right| .
$$

Com o cálculo feito anteriormente temos que a fibra $\widetilde{A}(K)$ do Hamiltoniano $\widetilde{H}_{f}$ é

$$
\widetilde{A}(K)=\underbrace{\epsilon\left(4-\cos \left(k_{\uparrow \downarrow}-K\right)-\cos (K)\right)+U_{0} P_{0}}_{A_{1,1}(K)}-\hat{w}\left(1+4 \mathrm{e}^{-2 \kappa}\right) P_{s+d} .
$$

Calculamos agora o autovalor $\tilde{E}(K)$ da fibra $\widetilde{A}(K)$, temos assim para a autofunção $[\tilde{\Psi}(K)]\left(k_{\uparrow \downarrow}\right)$ que

$$
\left(A_{1,1}(K)-\hat{w}\left(1+4 \mathrm{e}^{-2 \kappa}\right) P_{s+d}\right)[\tilde{\Psi}(K)]\left(k_{\uparrow \downarrow}\right)=\tilde{E}(K)[\tilde{\Psi}(K)]\left(k_{\uparrow \downarrow}\right) .
$$

Uma aplicação direta do principio de Birman-Schwinger, tal como foi feito para obter a equação (2.22), com

$$
H_{0}=A_{1,1}(K) \quad \text { e } \quad V=\hat{w}\left(1+4 \mathrm{e}^{-2 \kappa}\right) P_{s+d}
$$

nos da que $\lambda=\tilde{E}(K) \in \sigma_{d}(\widetilde{A}(K))$ se

$$
\hat{w} \mathcal{R}(k, \lambda)=1
$$

com $\mathcal{R}(k, \lambda)$ tal como é definido em $(2.23)$.

No limite $\epsilon \rightarrow 0, U_{0} / \varkappa>>1$ vimos que a autofunção de $A(K)$ tem só componente 
$d$-wave (2.31), portanto vamos impor sobre $\widetilde{A}(K)$ que sua autofunção neste limite seja a mesma de $A(k)$ e tenha o autovalor do estado fundamental.

Temos então para a autofunção $[\tilde{\Psi}(K)]\left(k_{\uparrow \downarrow}\right)$ de $\widetilde{A}(K)_{(\epsilon \rightarrow 0)}$,

$\widetilde{A}(K)_{(\epsilon \rightarrow 0)}[\tilde{\Psi}(K)]\left(k_{\uparrow \downarrow}\right)=\left(U_{0} P_{0}-\hat{w}\left(1+4 \mathrm{e}^{-2 \kappa}\right) P_{s+d}\right)[\tilde{\Psi}(K)]\left(k_{\uparrow \downarrow}\right)=\tilde{E}(K)[\tilde{\Psi}(K)]\left(k_{\uparrow \downarrow}\right)$

onde $\tilde{E}_{0}=\tilde{E}(K)$ com $K \in k_{m}=\{(0, \pm \pi),( \pm \pi, 0)\}$ corresponde a energia do estado fundamental. Em (2.36) calculamos o limite da energia do estado fundamental em ordem zero para $U_{0} / \varkappa>>1$, ate o seguente ordem obtemos ${ }^{1}$

$$
E_{0}=E(K) \rightarrow-\frac{\varkappa^{2}}{2 U_{0}}-2 \varkappa \mathrm{e}^{-\kappa} \quad K \in k_{m}=\{(0, \pm \pi),( \pm \pi, 0)\}
$$

Agora calculamos a forma de $\hat{w}$ para obter o autovalor $\tilde{E}_{0} \rightarrow E_{0}$ ate ordem $U_{0}^{-1}$, assim de $(2.34)$ e $(5.1)$, temos que

$$
\hat{w} \rightarrow-\frac{\varkappa^{2}}{E_{0}}=\frac{\varkappa \mathrm{e}^{\kappa}}{2\left(1+\frac{\varkappa}{4 U_{0} \mathrm{e}^{-\kappa}}\right)} \rightarrow \tilde{\gamma} \varkappa-\tilde{\gamma}^{2} \frac{\varkappa^{2}}{2 U_{0}}
$$

$\operatorname{com} \tilde{\gamma}=\mathrm{e}^{\kappa} / 2$.

Como é observado de (2.32) e (2.36), no limite $\epsilon \rightarrow 0, U_{0} / \varkappa>>1$ temos para a autofunção fermiônica do estado fundamental no espaço de momentos

$$
\left[\Psi_{\uparrow \downarrow}(K)\right]\left(k_{\uparrow \downarrow}\right) \rightarrow \frac{\varkappa}{U_{0}}+\cos \left(k_{\uparrow \downarrow}-K\right) .
$$

Para nosso Hamiltoniano fermiônico fibra efetivo $\widetilde{A}(K)$ obtemos no limite $\epsilon \rightarrow 0$,

${ }^{1}$ Observamos de (2.34) e (2.35) que para $\epsilon \rightarrow 0$ ate ordem $U_{0}^{-1}$, o autovalor $\lambda$ satisfaz a equação

$$
\frac{U_{0}}{\varkappa^{2}} \lambda^{2}+\left(1+4 \mathrm{e}^{-2 \kappa}\right) \lambda-4 \mathrm{e}^{-2 \kappa} U_{0}=0
$$

daqui temos que para $U_{0} / \varkappa>>1$

$$
\lambda \rightarrow-\frac{\varkappa^{2}}{2 U_{0}}-2 \varkappa \mathrm{e}^{-\kappa}
$$




$$
\begin{aligned}
& U_{0} / \varkappa>>1 \\
& \begin{aligned}
\widetilde{A}(K)_{(\epsilon \rightarrow 0)}\left(\frac{\varkappa}{U_{0}}+\cos \left(k_{\uparrow \downarrow}-K\right)\right)=\left(U_{0} P_{0}-\hat{w}\left(1+4 \mathrm{e}^{-2 \kappa}\right) P_{s+d}\right)\left(\frac{\varkappa}{U_{0}}+\cos \left(k_{\uparrow \downarrow}-K\right)\right) \\
\rightarrow-\left(\frac{\varkappa^{2}}{2 U_{0}}+2 \varkappa \mathrm{e}^{-\kappa}\right) \cos \left(k_{\uparrow \downarrow}-K\right)
\end{aligned}
\end{aligned}
$$

onde usamos a forma calculada anteriormente para $\hat{w}$ em (5.2). Assim constatamos que com a função $\hat{w}$ calculada reproduzimos corretamente a dinâmica do Hamiltoniano original no limite considerado.

Observamos que a função $\hat{w}$ tem picos nos pontos $K \in\{(0, \pm \pi),( \pm \pi, 0)\}$ se a função $\hat{v}$ tem também. Por causa disso é natural aproximar $w$ como

$$
w(x)=\sum_{k_{m}} \hat{w} \mathrm{e}^{-i k_{m} \cdot x}
$$

Notamos então que

$$
W^{\prime}=-\sum_{k_{m}, x, y \in \mathbb{Z}^{2}} \hat{w} \mathrm{e}^{-i k_{m} \cdot(x-y)} c_{x}^{\dagger} c_{y}=-\hat{w} \sum_{k_{m}}\left(\sum_{x \in \mathbb{Z}^{2}} \mathrm{e}^{i k_{m} \cdot x} c_{x}\right)^{\dagger} \underbrace{\left(\sum_{x \in \mathbb{Z}^{2}} \mathrm{e}^{i k_{m} \cdot x} c_{x}\right)}_{\hat{c}} .
$$

Observamos que se nos restringimos em um setor da rede $\Lambda \subset \mathbb{Z}^{2}$ podemos interpretar o operador

$$
\frac{1}{|\Lambda|} \sum_{x \in \Lambda} \mathrm{e}^{i k_{m} \cdot x} c_{x}
$$

como a média de um operador ou um campo médio.

Assim para $\Lambda \subset \mathbb{Z}^{2}$ resulta natural definir o operador de interação de campo médio

$$
\tilde{W}^{\prime}:=-\frac{1}{|\Lambda|} \sum_{x, y \in|\Lambda|} w(x-y) c_{x}^{\dagger} c_{y}=-\hat{w}|\Lambda| \sum_{k_{m}}\left(\frac{1}{|\Lambda|} \sum_{x \in \Lambda} \mathrm{e}^{i k_{m} \cdot x} c_{x}\right)^{\dagger}\left(\frac{1}{|\Lambda|} \sum_{x \in \Lambda} \mathrm{e}^{i k_{m} \cdot x} c_{x}\right)
$$

Podemos reconhecer em $\tilde{W}^{\prime}$ uma simetria de invariância por permutação de blocos na rede $^{2}$. Esta simetria pode ser usada para encontrar os estados de equilíbrio [4].

\footnotetext{
${ }^{2}$ Para ter simetria por permutação de blocos $(2 \times 2)$ na rede consideramos o caso de bipolarons locais,
} 
Como vimos a dinâmica do Hamiltoniano efetivo é uma aproximação que é valida no limite de baixa energia cinética e repulsão coulombiana forte comparada com a força da interação atrativa elétron-elétron efetiva, que é a região que interessa para o estudo das ondas de densidade. A simetria do Hamiltoniano efetivo é similar ao caso estudado em [4], pelo que um resultado importante deste trabalho é usado (Teorema 2.1). Num principio consideramos então o caso de acoplamento forte, depois se investigara o efeito do termo cinético que pode ser considerado por métodos perturbativos.

\section{Cálculo da Pressão Termodinâmica}

Para a analise do problema (principalmente o cálculo do limite termodinâmico da pressão) o termo de interação efetivo $\tilde{W}^{\prime}$ é desdobrado mediante o método do Hamiltoniano aproximado de Bogoliubov [3]. A simetria por permutação de blocos permite deduzir propriedades dos estados de equilíbrio, em particular eles podem ser escritos como estados produto [4]. Usando esta simetria e o método do Bogoliubov se calcula o limite termodinâmico da pressão de forma similar como é feito em [4]. Como foi mencionado, a diferença do caso estudado em [4], onde o termo de interação é invariante por permutação de pontos da rede, a interação efetiva (5.4) sera invariante por permutação de blocos da rede, o que é considerar que cada elétron tem mais graus de liberdade para um "ponto efetivo". Finalmente pode ver-se que numa região de parâmetros $\left(\mu, U_{0}, \hat{w}\right)$ a pressão de equilíbrio no limite termodinâmico se atinge quando se presentam ondas de densidade.

Como foi dito anteriormente na fase inicial consideramos o limite do acoplamento forte, ou seja não se toma no conta a parte cinética em (2.1), temos assim o Hamiltoniano de acoplamento forte ou "strong coupling"

$$
H_{s c} \doteq-\mu \sum_{s \in\{\uparrow, \downarrow\}, x \in \Lambda} a_{x, s}^{\dagger} a_{x, s}+U_{0} \sum_{x \in \Lambda} n_{x, \uparrow} n_{x, \downarrow}+\tilde{W}^{\prime}
$$

ou seja

$$
c_{x} \doteq a_{x, \uparrow} a_{x, \downarrow}
$$


O Hamiltoniano $H_{s c}$ é o Hamiltoniano considerado para fazer a aproximação mediante o método de Bogoliubov. Pela estrutura dos operadores de campo médio (5.3) o Hamiltoniano aproximado de Bogoliubov sera invariante por permutação de blocos da rede, como é mencionado acima. O problema de encontrar o limite termodinâmico da pressão se reduz assim a encontrar as constantes de Bogoliubov que minimizam a energia livre.

Como mencionamos acima o termo de interação $\tilde{W}^{\prime}$ em (5.5) é função dos operadores de campo médio. Assim podemos escrever nosso Hamiltoniano aproximado de Bogoliubov

$$
\begin{aligned}
H_{s c}\left(\mathfrak{b}_{1}, \mathfrak{b}_{2}, \mathfrak{b}_{3}, \mathfrak{b}_{4}\right) \doteq & -\mu \sum_{s \in\{\uparrow, \downarrow\}, x \in \Lambda} a_{x, s}^{\dagger} a_{x, s}+U_{0} \sum_{x \in \Lambda} n_{x, \uparrow} n_{x, \downarrow} \\
& -\hat{w} \sum_{m=1}^{4} \sum_{x \in \Lambda}\left(\left(\mathfrak{b}_{m}^{*} \mathrm{e}^{i k_{m} \cdot x} c_{x}\right)^{\dagger}+\mathfrak{b}_{m}^{*} \mathrm{e}^{i k_{m} \cdot x} c_{x}\right)
\end{aligned}
$$

onde as constantes $\mathfrak{b}_{m} \in \mathbb{C}$. Observamos que

$\hat{w}|\Lambda||\mathfrak{b}|^{2}+H_{s c}\left(\mathfrak{b}_{1}, \mathfrak{b}_{2}, \mathfrak{b}_{3}, \mathfrak{b}_{4}\right)-H_{s c}=\frac{\hat{w}}{|\Lambda|} \sum_{m=1}^{4}\left(\sum_{x \in \Lambda} \mathrm{e}^{i k_{m} \cdot x} c_{x}-|\Lambda| \mathfrak{b}_{m}\right)^{\dagger}\left(\sum_{x \in \Lambda} \mathrm{e}^{i k_{m} \cdot x} c_{x}-|\Lambda| \mathfrak{b}_{m}\right)$

onde $|\mathfrak{b}|^{2}=\sum_{m}\left|\mathfrak{b}_{m}\right|^{2}$

Temos a pressão na região $\Lambda$ definida por

$$
p_{\Lambda}\left(\mu, U_{0}, \hat{w}, \beta\right) \doteq \frac{1}{\beta|\Lambda|} \ln \operatorname{Tr}\left(\mathrm{e}^{-\beta H_{s c}}\right)
$$

Usando (5.7) e a desigualdade de Golden-Thompson $\operatorname{Tr}\left(\mathrm{e}^{A+B^{*} B}\right) \leq \operatorname{Tr}\left(\mathrm{e}^{A}\right)$ temos que

$$
p_{\Lambda}\left(\mu, U_{0}, \hat{w}, \beta\right) \geq \sup _{\mathfrak{b}_{1}, \mathfrak{b}_{2}, \mathfrak{b}_{3}, \mathfrak{b}_{4}}\left(-\hat{w}|\mathfrak{b}|^{2}+p\left(\mathfrak{b}_{1}, \mathfrak{b}_{2}, \mathfrak{b}_{3}, \mathfrak{b}_{4}\right)\right)
$$

onde

$$
p\left(\mathfrak{b}_{1}, \mathfrak{b}_{2}, \mathfrak{b}_{3}, \mathfrak{b}_{4}\right) \doteq \frac{1}{\beta|\Lambda|} \ln \operatorname{Tr}\left(\mathrm{e}^{-\beta H_{s c}\left(\mathfrak{b}_{1}, \mathfrak{b}_{2}, \mathfrak{b}_{3}, \mathfrak{b}_{4}\right)}\right)
$$


Pode ser provado [4] justamente que

$$
p_{\Lambda}\left(\mu, U_{0}, \hat{w}, \beta\right)=\sup _{\mathfrak{b}_{1}, \mathfrak{b}_{2}, \mathfrak{b}_{3}, \mathfrak{b}_{4}}\left(-\hat{w}|\mathfrak{b}|^{2}+p\left(\mathfrak{b}_{1}, \mathfrak{b}_{2}, \mathfrak{b}_{3}, \mathfrak{b}_{4}\right)\right)
$$

Agora o problema de encontrar a pressão de equilíbrio se traduz em um problema variacional dependente das constantes de Bogoliubov $\mathfrak{b}_{m}$.

Sendo a Hamiltoniana (5.6) uma soma de operadores locais que comutam (o que é a principal ventagem do Hamiltoniano aproximado de Bogoliubov) e a propriedade do traço para operadores fermiônicos (álgebra CAR) que atuam sobre regiões disjuntas [31] $\left(\operatorname{tr}(A B)=\operatorname{tr}(A) \operatorname{tr}(B)^{3}\right)$ temos que

$$
p\left(\mathfrak{b}_{1}, \mathfrak{b}_{2}, \mathfrak{b}_{3}, \mathfrak{b}_{4}\right)=\frac{1}{\beta|\Lambda|} \ln \operatorname{Tr}\left(\mathrm{e}^{-\beta H_{s c}\left(\mathfrak{b}_{1}, \mathfrak{b}_{2}, \mathfrak{b}_{3}, \mathfrak{b}_{4}\right)}\right)=\frac{1}{\beta\left|\Lambda_{b}\right|} \ln \operatorname{Tr}\left(\mathrm{e}^{-\beta H_{\Lambda_{b}}\left(\mathfrak{b}_{1}, \mathfrak{b}_{2}, \mathfrak{b}_{3}, \mathfrak{b}_{4}\right)}\right)
$$

onde $\Lambda_{b}$ é uma região que contem um bloco base.

$$
\begin{aligned}
H_{\Lambda_{b}}\left(\mathfrak{b}_{1}, \mathfrak{b}_{2}, \mathfrak{b}_{3}, \mathfrak{b}_{4}\right) \doteq & -\mu \sum_{s \in\{\uparrow, \downarrow\}, x \in \Lambda_{b}} a_{x, s}^{\dagger} a_{x, s}+U_{0} \sum_{x \in \Lambda_{b}} n_{x, \uparrow} n_{x, \downarrow} \\
& -\hat{w} \sum_{m=1}^{4} \sum_{x \in \Lambda_{b}}\left(\left(\mathfrak{b}_{m}^{*} \mathrm{e}^{i k_{m} \cdot x} c_{x}\right)^{\dagger}+\mathfrak{b}_{m}^{*} \mathrm{e}^{i k_{m} \cdot x} c_{x}\right)
\end{aligned}
$$

\section{Caso $d$-wave}

Para o caso em que a função de acoplamento efetiva (no espaço de momento) tem máximos em $k_{m} \in\{(0, \pm \pi),( \pm \pi, 0)\}$, e portanto a função do par fermiônico tem simetria $d$-wave no limite de forte acoplamento, notamos que temos só duas constantes de Bogoliubov independentes, assim

$$
\begin{aligned}
H_{\Lambda_{b}}\left(\mathfrak{b}_{1}, \mathfrak{b}_{2}\right)= & -\mu \sum_{s \in\{\uparrow, \downarrow\}, i=1}^{4} a_{i, s}^{\dagger} a_{i, s}+U_{0} \sum_{i=1}^{4} n_{i, \uparrow} n_{i, \downarrow} \\
& -\hat{w} \sum_{m=1}^{2} \sum_{j=1}^{4}\left(\left(\mathfrak{b}_{m}^{*} \mathrm{e}^{i k_{m} \cdot x_{j}} c_{j}\right)^{\dagger}+\mathfrak{b}_{m}^{*} \mathrm{e}^{i k_{m} \cdot x_{j}} c_{j}\right) .
\end{aligned}
$$

\footnotetext{
${ }^{3} \operatorname{tr}(\cdot)=\frac{\operatorname{Tr}(\cdot)}{\operatorname{Tr}(\mathbb{1})}$ é o traço normalizado.
} 
onde $k_{1}=(0, \pi), k_{2}=(\pi, 0)$ e $x_{j} \in\{(0,0),(0,1),(1,0),(1,1)\}$. Agora temos que

$$
\ln \operatorname{Tr}\left(\mathrm{e}^{-\beta H_{\Lambda_{b}}\left(\mathfrak{b}_{1}, \mathfrak{b}_{2}, \mathfrak{b}_{3}, \mathfrak{b}_{4}\right)}\right)=\sum_{i=1}^{4} \ln \operatorname{Tr}\left(\mathrm{e}^{-\beta H_{i}}\right)
$$

onde

$$
\begin{gathered}
H_{i} \doteq-\mu \sum_{s \in\{\uparrow, \downarrow\}} a_{i, s}^{\dagger} a_{i, s}+U_{0} n_{i, \uparrow} n_{i, \downarrow}-\hat{w}\left(\tilde{\mathfrak{b}}_{i}^{*} c_{i}^{\dagger}+\tilde{\mathfrak{b}}_{i} c_{i}\right) \\
\tilde{\mathfrak{b}}_{i} \doteq \sum_{m=1}^{2} \mathfrak{b}_{m}^{*} \mathrm{e}^{i k_{m} \cdot x_{i}} .
\end{gathered}
$$

Para os Hamiltonianos locais $H_{i}$, definimos as constantes $\eta$ e $\tilde{\eta}$

$$
\eta:=\left(\mathfrak{b}_{1}+\mathfrak{b}_{2}\right)^{*}
$$

e

$$
\tilde{\eta}:=\left(\mathfrak{b}_{1}-\mathfrak{b}_{2}\right)^{*}
$$

assim temos que

$$
\begin{aligned}
& H_{1} \doteq-\mu \sum_{s \in\{\uparrow, \downarrow\}} a_{1, s}^{\dagger} a_{1, s}+U_{0} n_{1, \uparrow} n_{1, \downarrow}-\hat{w}\left(\eta^{*} c_{1}^{\dagger}+\eta c_{1}\right) \\
& H_{2} \doteq-\mu \sum_{s \in\{\uparrow, \downarrow\}} a_{2, s}^{\dagger} a_{2, s}+U_{0} n_{2, \uparrow} n_{2, \downarrow}-\hat{w}\left(\tilde{\eta}^{*} c_{2}^{\dagger}+\tilde{\eta} c_{2}\right) \\
& H_{3} \doteq-\mu \sum_{s \in\{\uparrow, \downarrow\}} a_{3, s}^{\dagger} a_{3, s}+U_{0} n_{3, \uparrow} n_{3, \downarrow}+\hat{w}\left(\tilde{\eta}^{*} c_{3}^{\dagger}+\tilde{\eta} c_{3}\right) \\
& H_{4} \doteq-\mu \sum_{s \in\{\uparrow, \downarrow\}} a_{4, s}^{\dagger} a_{4, s}+U_{0} n_{4, \uparrow} n_{4, \downarrow}+\hat{w}\left(\eta^{*} c_{4}^{\dagger}+\eta c_{4}\right)
\end{aligned}
$$




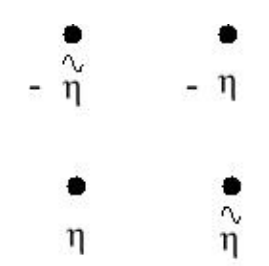

Figura 5.1: Distribuição das constantes $\pm \eta$ e $\pm \tilde{\eta}$ num bloco base.

Agora calculamos a Traça do Hamiltoniano aproximado para um ponto da rede

$$
\operatorname{Tr}\left(\mathrm{e}^{-\beta H_{1}}\right)=\operatorname{Tr}\left(\mathrm{e}^{-\beta\left(-\mu \sum_{s \in\{\uparrow, \downarrow\}} a_{1, s}^{\dagger} a_{1, s}+U_{0} n_{1, \uparrow} n_{1, \downarrow}-\hat{w}\left(\eta^{*} c_{1}^{\dagger}+\eta c_{1}\right)\right)}\right)
$$

como o espaço do sistema num sitio da rede é gerado pela base $\{|0\rangle,|\uparrow\rangle,|\downarrow\rangle,|\uparrow \downarrow\rangle\}$ calculamos agora a ação de $H_{1}$ sobre cada termo da base, assim

$$
\begin{gathered}
\left(-\mu \sum_{s \in\{\uparrow, \downarrow\}} a_{1, s}^{\dagger} a_{1, s}+U_{0} n_{1, \uparrow} n_{1, \downarrow}-\hat{w}\left(\eta^{*} c_{1}^{\dagger}+\eta c_{1}\right)\right)|0\rangle=-\hat{w} \eta^{*}|\uparrow \downarrow\rangle \\
\left(-\mu \sum_{s \in\{\uparrow, \downarrow\}} a_{1, s}^{\dagger} a_{1, s}+U_{0} n_{1, \uparrow} n_{1, \downarrow}-\hat{w}\left(\eta^{*} c_{1}^{\dagger}+\eta c_{1}\right)\right)|\uparrow\rangle=-\mu|\uparrow\rangle \\
\left(-\mu \sum_{s \in\{\uparrow, \downarrow\}} a_{1, s}^{\dagger} a_{1, s}+U_{0} n_{1, \uparrow} n_{1, \downarrow}-\hat{w}\left(\eta^{*} c_{1}^{\dagger}+\eta c_{1}\right)\right)|\downarrow\rangle=-\mu|\downarrow\rangle \\
\left(-\mu \sum_{s \in\{\uparrow, \downarrow\}} a_{1, s}^{\dagger} a_{1, s}+U_{0} n_{1, \uparrow} n_{1, \downarrow}-\hat{w}\left(\eta^{*} c_{1}^{\dagger}+\eta c_{1}\right)\right)|\uparrow \downarrow\rangle=\left(-2 \mu+U_{0}\right)|\uparrow \downarrow\rangle-\hat{w} \eta|0\rangle .
\end{gathered}
$$

Temos então os elementos de matriz $\left\langle i\left|H_{1}\right| j\right\rangle$ do Hamiltoniano de Bogoliubov

$$
\mathbb{H}_{1}=\left(\begin{array}{cccc}
0 & 0 & 0 & -\hat{w} \eta^{*} \\
0 & -\mu & 0 & 0 \\
0 & 0 & -\mu & 0 \\
-\hat{w} \eta & 0 & 0 & -2 \mu+U_{0}
\end{array}\right)
$$

calculamos agora os autovalores de $\mathbb{H}_{1}$ com o fim de fazer os cálculos da pressão na base 
que diagonaliza o Hamiltoniano efetivo. Temos então que

$$
\operatorname{det}\left(\mathbb{H}_{1}-\lambda \mathbb{1}\right)=0 \Longrightarrow(\mu+\lambda)^{2}\left[\left(\lambda^{2}-\left(-2 \mu+U_{0}\right) \lambda-\hat{w}^{2}|\eta|^{2}\right)\right]=0
$$

assim o conjunto de autovalores é $\left\{-\mu,-\mu, \varsigma+\sqrt{\varsigma^{2}+\hat{w}^{2}|\eta|^{2}}, \varsigma-\sqrt{\varsigma^{2}+\hat{w}^{2}|\eta|^{2}}\right\}$, onde

$$
\varsigma:=\frac{-2 \mu+U_{0}}{2}
$$

Observamos que

$$
\operatorname{Tr}\left(\mathrm{e}^{-\beta H_{1}}\right)=2 \mathrm{e}^{\beta \mu}\left(1+\mathrm{e}^{-\frac{\beta U_{0}}{2}} \cosh \left(\beta \sqrt{\varsigma^{2}+\hat{w}^{2}|\eta|^{2}}\right)\right)
$$

assim notamos

$$
\begin{aligned}
\frac{\sum_{i=1}^{4} \ln \operatorname{Tr}\left(\mathrm{e}^{-\beta H_{i}}\right)}{2}=\ln 4+2 \mu \beta+\ln \left(1+\mathrm{e}^{-\frac{\beta U_{0}}{2}}\right. & \left.\cosh \left(\beta \sqrt{\varsigma^{2}+\hat{w}^{2}|\eta|^{2}}\right)\right) \\
& +\ln \left(1+\mathrm{e}^{-\frac{\beta U_{0}}{2}} \cosh \left(\beta \sqrt{\varsigma^{2}+\hat{w}^{2}|\tilde{\eta}|^{2}}\right)\right)
\end{aligned}
$$

e temos para (5.8) que

$$
p_{\Lambda}\left(\mu, U_{0}, \hat{w}, \beta\right)=\frac{\ln 2}{\beta}+\mu+\sup _{\mathfrak{b}_{1}, \mathfrak{b}_{2}} \mathfrak{f}\left(|\eta|^{2},|\tilde{\eta}|^{2}\right)
$$

com

$\mathfrak{f}(x, y)=-\hat{w} \frac{x+y}{2}+\frac{1}{2 \beta}\left(\ln \left(1+\mathrm{e}^{-\frac{\beta U_{0}}{2}} \cosh \left(\beta \sqrt{\varsigma^{2}+\hat{w}^{2} x}\right)\right)+\ln \left(1+\mathrm{e}^{-\frac{\beta U_{0}}{2}} \cosh \left(\beta \sqrt{\varsigma^{2}+\hat{w}^{2} y}\right)\right)\right)$

$\mathrm{Ou}$

$$
\mathfrak{f}\left(x_{1}, x_{2}\right)=\frac{1}{2} \sum_{i=1}^{2}\left(-\hat{w} x_{i}+\frac{1}{\beta} \ln \left(1+\mathrm{e}^{-\frac{\beta U_{0}}{2}} \cosh \left(\beta \sqrt{\varsigma^{2}+\hat{w}^{2} x_{i}}\right)\right) .\right.
$$




\section{Caso $p$-wave}

Se agora consideramos que os máximos da função de acoplamento $\hat{v}(k)$ estão localizados em $k_{m} \in\{(0, \pm \pi / 2),( \pm \pi / 2,0)\}$ a simetria por permutação de blocos na rede sera dada para blocos $4 \times 4$, assim notamos que teremos quatro constantes de Bogoliubov independentes (ver (5.6)). Para os Hamiltonianos locais temos

$$
H_{i} \doteq-\mu \sum_{s \in\{\uparrow, \downarrow\}} a_{i, s}^{\dagger} a_{i, s}+U_{0} n_{i, \uparrow} n_{i, \downarrow}-\hat{w}\left(\tilde{\mathfrak{b}}_{i}^{*} c_{i}^{\dagger}+\tilde{\mathfrak{b}}_{i} c_{i}\right)
$$

com

$$
\begin{gathered}
\tilde{\mathfrak{b}}_{i} \doteq \sum_{m=1}^{4} \mathfrak{b}_{m}^{*} \mathrm{e}^{i k_{m} \cdot x_{i}} \\
k_{1}=(0, \pi / 2), k_{2}=(0,-\pi / 2), k_{3}=(\pi / 2,0), k_{4}=(-\pi / 2,0)
\end{gathered}
$$

e

$$
x_{i} \in\{(n, m)|n, m \geq 0,|(n, m) \mid \leq 3\}
$$

definimos as constantes $\eta_{i}:=\tilde{\mathfrak{b}}_{i}$,

$$
\begin{gathered}
\eta_{1}=\left(\mathfrak{b}_{1}+\mathfrak{b}_{2}+\mathfrak{b}_{3}+\mathfrak{b}_{4}\right)^{*} \\
\eta_{2}=\left(\mathfrak{b}_{1}+\mathfrak{b}_{2}-i \mathfrak{b}_{3}+i \mathfrak{b}_{4}\right)^{*} \\
\eta_{3}=\left(\mathfrak{b}_{1}+\mathfrak{b}_{2}-\mathfrak{b}_{3}-\mathfrak{b}_{4}\right)^{*} \\
\eta_{4}=\left(\mathfrak{b}_{1}+\mathfrak{b}_{2}+i \mathfrak{b}_{3}-i \mathfrak{b}_{4}\right)^{*} \\
\eta_{5}=\left(-i \mathfrak{b}_{1}+i \mathfrak{b}_{2}+\mathfrak{b}_{3}+\mathfrak{b}_{4}\right)^{*} \\
\eta_{6}=\left(-i \mathfrak{b}_{1}+i \mathfrak{b}_{2}-i \mathfrak{b}_{3}+i \mathfrak{b}_{4}\right)^{*} \\
\eta_{7}=\left(-i \mathfrak{b}_{1}+i \mathfrak{b}_{2}-\mathfrak{b}_{3}-\mathfrak{b}_{4}\right)^{*} \\
\eta_{8}=\left(-i \mathfrak{b}_{1}+i \mathfrak{b}_{2}+i \mathfrak{b}_{3}-i \mathfrak{b}_{4}\right)^{*}
\end{gathered}
$$


e a pressão de equilíbrio sera dada por

$$
p_{\Lambda}\left(\mu, U_{0}, \hat{w}, \beta\right)=\frac{\ln 2}{\beta}+\mu+\sup _{\mathfrak{b}_{1}, \mathfrak{b}_{2}, \mathfrak{b}_{3}, \mathfrak{b}_{4}} \mathfrak{g}\left(|\mathfrak{b}|^{2},\left|\eta_{1}\right|^{2},\left|\eta_{2}\right|^{2},\left|\eta_{3}\right|^{2},\left|\eta_{4}\right|^{2},\left|\eta_{5}\right|^{2},\left|\eta_{6}\right|^{2},\left|\eta_{7}\right|^{2},\left|\eta_{8}\right|^{2}\right)
$$

com

$\mathfrak{g}\left(x_{1}, x_{2}, x_{3}, x_{4}, x_{5}, x_{6}, x_{7}, x_{8}, x_{9}\right):=-\hat{w} x_{1}+\frac{1}{8 \beta}\left(\sum_{i=2}^{9} \ln \left(1+\mathrm{e}^{-\frac{\beta U_{0}}{2}} \cosh \left(\beta \sqrt{\varsigma^{2}+\hat{w}^{2} x_{i}}\right)\right)\right)$
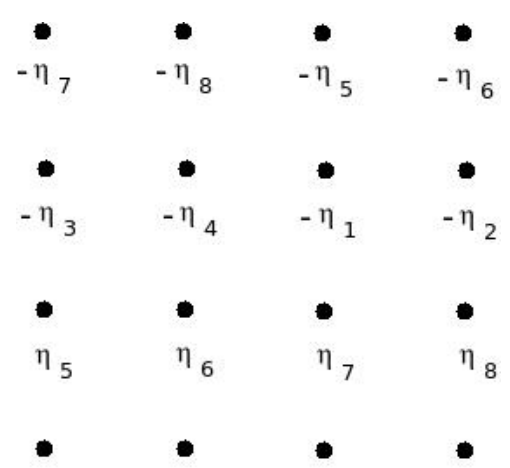

$\eta_{1} \quad \eta_{2} \quad \eta_{3} \quad \eta_{4}$

Figura 5.2: Ilustração da distribuição das constantes $\eta_{i}$ para cada ponto de um bloco base para o caso $p$-wave.

Notamos que as constantes $\mathfrak{b}_{1}$ e $\mathfrak{b}_{2}$ estão associadas aos picos da função de interação no eixo horizontal do espaço de momento, e respetivamente assim para as constantes $\mathfrak{b}_{3}$ e $\mathfrak{b}_{4}$ para o eixo vertical. Definimos assim as constantes

$$
\begin{gathered}
z_{h}^{+}:=\mathfrak{b}_{1}+\mathfrak{b}_{2} \\
z_{h}^{-}:=i\left(\mathfrak{b}_{1}-\mathfrak{b}_{2}\right) \\
z_{v}^{+}:=\mathfrak{b}_{3}+\mathfrak{b}_{4} \\
z_{v}^{-}:=i\left(\mathfrak{b}_{3}-\mathfrak{b}_{4}\right)
\end{gathered}
$$

e podemos escrever

$$
\left|\eta_{1}\right|=\left|z_{h}^{+}+z_{v}^{+}\right|
$$




$$
\begin{aligned}
& \left|\eta_{2}\right|=\left|z_{h}^{+}-z_{v}^{-}\right| \\
& \left|\eta_{3}\right|=\left|z_{h}^{+}-z_{v}^{+}\right| \\
& \left|\eta_{4}\right|=\left|z_{h}^{+}+z_{v}^{-}\right| \\
& \left|\eta_{5}\right|=\left|z_{v}^{+}-z_{h}^{-}\right| \\
& \left|\eta_{6}\right|=\left|z_{h}^{-}+z_{v}^{-}\right| \\
& \left|\eta_{7}\right|=\left|z_{h}^{-}+z_{v}^{+}\right| \\
& \left|\eta_{8}\right|=\left|z_{v}^{-}-z_{h}^{-}\right| .
\end{aligned}
$$

Notamos que

$$
\sum_{i=1}^{8}\left|\eta_{i}\right|^{2}=4\left(\left|z_{h}^{+}\right|^{2}+\left|z_{h}^{-}\right|^{2}+\left|z_{v}^{+}\right|^{2}+\left|z_{v}^{-}\right|^{2}\right)
$$

e que

$$
|\mathfrak{b}|^{2}=\sum_{m=1}^{4}\left|\mathfrak{b}_{m}\right|^{2}=\frac{1}{4}\left(\left|z_{h}^{+}+i z_{h}^{-}\right|^{2}+\left|z_{h}^{+}-i z_{h}^{-}\right|^{2}+\left|z_{v}^{+}+i z_{v}^{-}\right|^{2}+\left|z_{v}^{+}-i z_{v}^{-}\right|^{2}\right)=\frac{1}{8} \sum_{i=1}^{8}\left|\eta_{i}\right|^{2}
$$

portanto a pressão (5.13) fica desacoplada numa soma de termos similar ao caso d-wave.

Assim podemos escrever para o caso $p$-wave

$$
p_{\Lambda}\left(\mu, U_{0}, \hat{w}, \beta\right)=\frac{\ln 2}{\beta}+\mu+\sup _{\mathfrak{b}_{1}, \mathfrak{b}_{2}, \mathfrak{b}_{3}, \mathfrak{b}_{4}} \tilde{\mathfrak{g}}\left(\left|\eta_{1}\right|^{2},\left|\eta_{2}\right|^{2},\left|\eta_{3}\right|^{2},\left|\eta_{4}\right|^{2},\left|\eta_{5}\right|^{2},\left|\eta_{6}\right|^{2},\left|\eta_{7}\right|^{2},\left|\eta_{8}\right|^{2}\right)
$$

com

$$
\tilde{\mathfrak{g}}\left(x_{1}, x_{2}, x_{3}, x_{4}, x_{5}, x_{6}, x_{7}, x_{8}\right)=\frac{1}{8} \sum_{i=1}^{8}\left(-\hat{w} x_{i}+\frac{1}{\beta} \ln \left(1+\mathrm{e}^{-\frac{\beta U_{0}}{2}} \cosh \left(\beta \sqrt{\varsigma^{2}+\hat{w}^{2} x_{i}}\right)\right) .\right.
$$




\section{Condição no Limite de Baixas Temperaturas para Existência de Ondas de Densidade no Caso $d$ - wave e $p$-wave}

Nesta secção vamos mostrar a condição para a existência de ondas de densidade para o caso no que a função de acoplamento presenta picos em $\left(k_{1}, k_{2}\right) \in\{(0, \pm \pi),( \pm \pi, 0)\}$ (caso $d$-wave) ou $\left(k_{1}, k_{2}\right) \in\{(0, \pm \pi / 2),( \pm \pi / 2,0)\}$ (caso $p$-wave). A aproximação a ser usada sera o limite de baixa temperatura $(\beta>>1)$.

Como foi mostrado na secção anterior temos no problema variacional para encontrar a pressão de equilíbrio, uma soma de funções do tipo

$$
f(r)=-\gamma r+\frac{1}{\beta} \ln \left(1+\mathrm{e}^{-\lambda \beta} \cosh \left(\beta g_{r}\right)\right)
$$

$\operatorname{com} g_{r}=\sqrt{(\mu-\lambda)^{2}+\gamma^{2} r}, \gamma=\hat{w}$ e $\lambda=U_{0} / 2$.

A condição $\partial_{r} f(r)=0$ conduz a equação

$$
\tanh \left(\beta g_{r_{\beta}}\right)=\frac{2 g_{r_{\beta}}}{\gamma}\left(1+\frac{\mathrm{e}^{\lambda \beta}}{\cosh \left(\beta g_{r_{\beta}}\right)}\right)
$$

que também pode ser escrita assim

$$
\mathfrak{K}(x)=x \operatorname{com} \mathfrak{K}(x):=\frac{\gamma \beta}{2} \frac{\tanh (x)}{1+\mathrm{e}^{\lambda \beta} \operatorname{sech}(x)}
$$

$\operatorname{com} x=\beta g_{r_{\beta}}$.

Para um $\beta>>1$ se tem

$$
\frac{\tanh (x)}{1+\mathrm{e}^{\lambda \beta} \operatorname{sech}(x)} \rightarrow \Theta^{\beta}(x)
$$

com

$$
\Theta^{\beta}(x)=\frac{1}{1+\mathrm{e}^{\lambda \beta-x+\ln 2}}=\frac{1}{2}+\frac{1}{2} \tanh (\beta(\tilde{\mathfrak{r}}-\mathfrak{r}))
$$


$\operatorname{com} \tilde{\mathfrak{r}}=\frac{g_{r_{\beta}}}{2}$ e $\mathfrak{r}=\frac{\lambda}{2}+\frac{\ln 2}{2 \beta}$.

Notamos que conforme $\beta \rightarrow \infty$ a função $\Theta^{\beta}(x)$ se aproxima a uma função de Heaviside, assim

$$
\lim _{\beta \rightarrow \infty} \Theta^{\beta}(x)=H\left(\tilde{\mathfrak{r}}-\mathfrak{r}^{\prime}\right)
$$

$\operatorname{com} \mathfrak{r}^{\prime}=\lambda / 2 \mathrm{e}$

$$
H\left(\tilde{\mathfrak{r}}-\mathfrak{r}^{\prime}\right)=\left\{\begin{array}{lll}
0 & \text { se } & \tilde{\mathfrak{r}}<\mathfrak{r}^{\prime} \\
1 & \text { se } & \tilde{\mathfrak{r}}>\mathfrak{r}^{\prime}
\end{array}\right.
$$

Assim a equação (5.16) no limite $\beta \rightarrow \infty$ pode ser escrita como

$$
\frac{\gamma}{2} H\left(\tilde{\mathfrak{r}}-\mathfrak{r}^{\prime}\right)=2 \tilde{\mathfrak{r}}
$$

Então neste caso $(\beta \rightarrow \infty)$ é simples notar de (5.19) que a função (5.15) sera decrescente para $0<r<r_{\beta}^{-}$, onde $r_{\beta}^{-}$corresponde ao menor solução de $(5.19)^{4}$. A partir do ponto $r_{\beta}^{-}$a função (5.15) será crescente ate o ponto $r_{\beta}^{+}$, e para $r>r_{\beta}^{+}$sera novamente decrescente. No caso em que existam $r_{\beta}^{-}, r_{\beta}^{+}>0$ notamos que os parâmetros físicos de (5.15) podem "ajustar-se" para que existam dois maximantes (0 e $\left.r_{\beta}^{+}\right)$tal que $f(0)=f\left(r_{\beta}^{+}\right)$. Notamos agora que as condições para que existam duas soluções $r_{\beta}^{-}$e $r_{\beta}^{+}$, maiores que zero, da equação (5.19), $\operatorname{com} r_{\beta}^{-}<r_{\beta}^{+}$, são

$$
g_{r_{\beta}^{-}}<\frac{\gamma}{2} \text { e } r_{\beta}^{-}>0
$$

Observamos que $g_{r_{\beta}^{-}}=2 \mathfrak{r}^{\prime}$, ou seja

$$
r_{\beta}^{-}=\frac{\mu(2 \lambda-\mu)}{\gamma^{2}}
$$

e as condições (5.20) ficam resumidas como

$$
\frac{\mu}{2}<\lambda<\frac{\gamma}{2} \text { se } \mu>0
$$

\footnotetext{
${ }^{4} \mathrm{em}$ caso de existir duas soluções $r_{\beta}^{-}$e $r_{\beta}^{+}$.
} 
o caso $\mu<0$ implicaria $\lambda<0$, se consideramos $\lambda>0$ então descartamos a possibilidade $\mu<0$.

A segunda solução $r_{\beta}^{+}$satisfaz a equação

$$
g_{r_{\beta}^{+}}=\frac{\gamma}{2}
$$

$\mathrm{Ou}$

$$
r_{\beta}^{+}=\frac{1}{4}-\frac{(\mu-\lambda)^{2}}{\gamma^{2}}
$$

Para que existam ondas de densidade devem existir duas soluções para o maximizante, assim como mencionamos anteriormente, se deve satisfazer a condição

$$
f(0)=f\left(r_{\beta}^{+}\right)
$$

$\mathrm{Ou}$

$$
\gamma\left(\frac{1}{4}-\frac{(\mu-\lambda)^{2}}{\gamma^{2}}\right)=\frac{1}{\beta}\left(\ln \left(1+\mathrm{e}^{-\lambda \beta} \cosh \frac{\gamma}{2}\right)-\ln \left(1+\mathrm{e}^{-\lambda \beta} \cosh \beta|\mu-\lambda|\right)\right)
$$

no limite $\beta \rightarrow \infty$

$$
\begin{gathered}
\frac{1}{\beta} \ln \left(1+\mathrm{e}^{-\lambda \beta} \cosh \beta|\mu-\lambda|\right) \rightarrow \frac{1}{\beta} \ln \left(1+\frac{\mathrm{e}^{\beta(|\mu-\lambda|-\lambda)}}{2}\right) \rightarrow 0 \\
\frac{1}{\beta} \ln \left(1+\mathrm{e}^{-\lambda \beta} \cosh \frac{\gamma}{2}\right) \rightarrow \frac{1}{\beta} \ln \left(1+\frac{\mathrm{e}^{\beta\left(\frac{\gamma}{2}-\lambda\right)}}{2}\right) \rightarrow \frac{\gamma}{2}-\lambda
\end{gathered}
$$

sempre que $|\mu-\lambda|<\lambda$ e $0<\lambda<\frac{\gamma}{2}$, mas a condição (5.21) implica as ultimas.

Assim no limite $\beta \rightarrow \infty$ a equação (5.22) fica como

$$
\frac{(\mu-\lambda)^{2}}{\gamma^{2}}=\frac{\lambda}{\gamma}-\frac{1}{4}
$$

$\mathrm{Ou}$

$$
\mu=\lambda \pm \gamma \sqrt{\frac{\lambda}{\gamma}-\frac{1}{4}}
$$


observamos que no intervalo onde a equação (5.23) tem solução real e a condição (5.21) é respeitada, ou seja

$$
\frac{1}{4} \leq \frac{\lambda}{\gamma}<\frac{1}{2}
$$

o potencial químico dado por (5.23) barre tudo o intervalo permitido pela condição (5.21), ou seja $\mu \in\left(0,2 \lambda_{\max }\right)$, com $\lambda_{\max }=\gamma / 2$.

Assim as condições para que existam dois soluções para maximizar a pressão de equilíbrio, e portanto exista a solução com ondas de densidade, no caso $d, p$-wave para $\beta \rightarrow \infty$ vem dadas por (5.23) e (5.24).
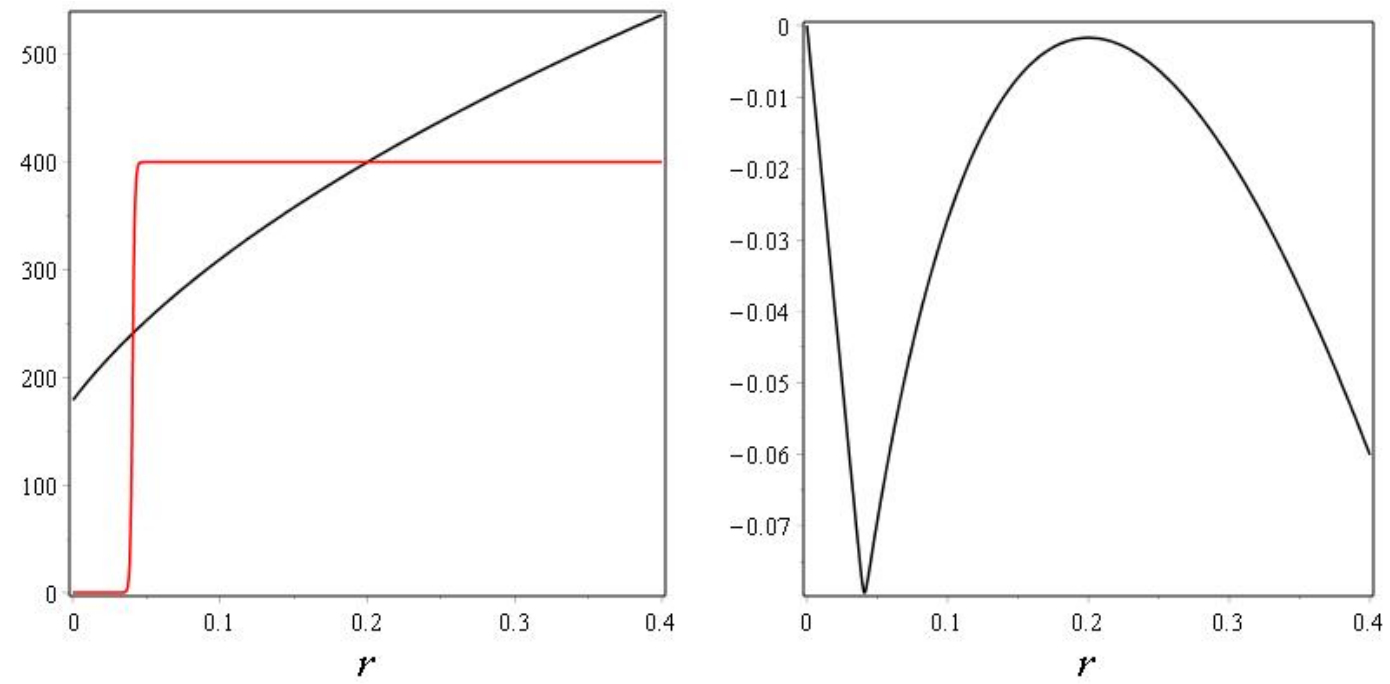

Figura 5.3: (esquerda) Função $\beta g_{r}$ (curva preta) e função $\mathfrak{K}\left(\beta g_{r}\right)$ (5.17) (curva vermelha) para $\lambda / \gamma=0.3, \gamma=2, \beta=400$ e $\mu=\lambda-\gamma \sqrt{\frac{\lambda}{\gamma}-\frac{1}{4}} \approx 0.15$; (direta) função $f(r)$ para os mesmos valores de parâmetros.

\section{Ondas de Densidade}

Como mostramos na secção anterior existe uma região de parâmetros $\left(\mu, U_{0}, \hat{w}\right)$ (ver (5.5)) onde a pressão termodinâmica de equilíbrio, calculada mediante o Hamiltoniano aproximado de Bogoliubov (5.6), apresenta duas soluções ou conjunto de contastes de Bogoliubov possíveis. Nesta região de parâmetros temos então uma família de soluções onde a "pressão local" oscila na rede $\mathbb{Z}^{2}$. Calcularemos agora a densidade eletrônica local 
para este tipo de soluções, mostrando assim que nosso modelo prediz a formação de ondas de densidade em uma família de estados de equilíbrio.

Como é provado em [4], os estados de equilíbrio para um ponto da rede (em nosso caso um bloco base) vem dados por a dinâmica do Hamiltoniano aproximado de Bogoliubov com as constantes $\mathfrak{b}_{m}(5.6)$ que atingem a pressão de equilíbrio. Assim para o caso $d$-wave temos os estados de Bogoliubov dados por

$$
\Omega_{\eta, \tilde{\eta}}(\cdot)=\frac{\operatorname{Tr}\left(\cdot \mathrm{e}^{-\beta H_{\Lambda_{b}}}\right)}{\operatorname{Tr}\left(\mathrm{e}^{-\beta H_{\Lambda_{b}}}\right)}
$$

com $H_{\Lambda_{b}}$ dado por (5.10). Vemos que o estado $\Omega_{\eta, \tilde{\eta}}(\cdot)$ atua sobre elementos $A \in \mathcal{U}_{\Lambda_{b}}$, onde $\mathcal{U}_{\Lambda_{b}}$ é a álgebra $C^{*}$ gerada por $\left\{a_{x, \uparrow}, a_{x, \downarrow}, \mathbb{1}\right\}$ com $x \in \Lambda_{b}$. Notamos também que o estado $\Omega_{\eta, \tilde{\eta}}(\cdot)$ é um estado produto, ou seja se $A_{i} \in \mathcal{U}_{i} \operatorname{com} \mathcal{U}_{i}$ a sub-álgebra $C^{*}$ local gerada por $\left\{a_{i, \uparrow}, a_{i, \downarrow}, \mathbb{1}\right\}$ com $i$ sendo um ponto pertence-te ao bloco base $\Lambda_{b}$, temos que

$$
\Omega_{\eta, \tilde{\eta}}\left(\prod_{i} A_{i}\right)=\prod_{i} \Omega^{i}\left(A_{i}\right)
$$

com

$$
\Omega^{i}(\cdot)=\frac{\operatorname{Tr}\left(\cdot \mathrm{e}^{-\beta H_{i}}\right)}{\operatorname{Tr}\left(\mathrm{e}^{-\beta H_{i}}\right)} .
$$

Vemos então que o valor médio da densidade eletrônica em um ponto $i \in \Lambda_{b}$ é

$$
\Omega_{\eta, \tilde{\eta}}\left(n_{i, \uparrow}+n_{i, \downarrow}\right)=\Omega^{i}\left(n_{i, \uparrow}+n_{i, \downarrow}\right)=\partial_{\mu} \tilde{p}_{i}(\mu, \lambda, \gamma, \beta)
$$

com

$$
\tilde{p}_{i}(\mu, \lambda, \gamma, \beta)=\frac{1}{\beta} \ln \operatorname{Tr}\left(\mathrm{e}^{-\beta H_{i}}\right)
$$

De (5.11) temos que

$$
\tilde{p}_{i}(\mu, \lambda, \gamma, \beta)=\frac{\ln 2}{\beta}+\mu+\frac{1}{\beta} \ln \left(1+\mathrm{e}^{-\lambda \beta} \cosh \left(\beta g_{\eta}\right)\right)
$$


e

$$
\partial_{\mu} \tilde{p}_{i}(\mu, \lambda, \gamma, \beta)=1+\frac{\tanh \left(\beta g_{\eta}\right)}{1+\mathrm{e}^{\lambda \beta} \operatorname{sech}\left(\beta g_{\eta}\right)} \cdot \frac{(\mu-\lambda)}{g_{\eta}}
$$

Como vimos na secção anterior, no limite de baixas temperaturas temos que

$$
\delta_{i}^{e}:=\partial_{\mu} \tilde{p}_{i}(\mu, \lambda, \gamma, \beta) \rightarrow 1+\frac{(\mu-\lambda)}{g_{\eta}} H\left(\tilde{\mathfrak{r}}-\mathfrak{r}^{\prime}\right)
$$

$\operatorname{com} \tilde{\mathfrak{r}}=\frac{g_{\eta}}{2}, \mathfrak{r}^{\prime}=\frac{\lambda}{2} \mathrm{e}$

$$
H\left(\tilde{\mathfrak{r}}-\mathfrak{r}^{\prime}\right)=\left\{\begin{array}{lll}
0 & \text { se } & \tilde{\mathfrak{r}}<\mathfrak{r}^{\prime} \\
& & \\
1 & \text { se } & \tilde{\mathfrak{r}}>\mathfrak{r}^{\prime}
\end{array}\right.
$$

Tal como foi discutido antes, os dois possíveis valores para a constante $\eta$ na pressão de equilíbrio são $\eta=r_{\beta}^{+}$e $\eta=0$, sempre que se esteja na região de parâmetros

$$
\begin{gathered}
\frac{1}{4} \leq \frac{\lambda}{\gamma}<\frac{1}{2} \\
\mu=\lambda \pm \gamma \sqrt{\frac{\lambda}{\gamma}-\frac{1}{4}}
\end{gathered}
$$

Para $\eta=r_{\beta}^{+}$temos $g_{r_{\beta}^{+}}=\frac{\gamma}{2}$, como $\gamma / 4 \leq \lambda<\gamma / 2$, esto implica que

$$
\delta_{i}^{e}=1+2 \frac{(\mu-\lambda)}{\gamma} H\left(\frac{\gamma}{2}-\lambda\right)=1 \pm 2 \sqrt{\frac{\lambda}{\gamma}-\frac{1}{4}}
$$

o que implica que $\delta_{i}^{e} \in(0,2)$.

Agora para a outra solução $\eta^{-}=0$ temos $g_{0}=|\mu-\lambda|$ e

$$
\delta_{i}^{e}=1+\operatorname{sgn}(\mu-\lambda) H(|\mu-\lambda|-\lambda)=1
$$

devido a condição de que $0<\mu<2 \lambda$. 


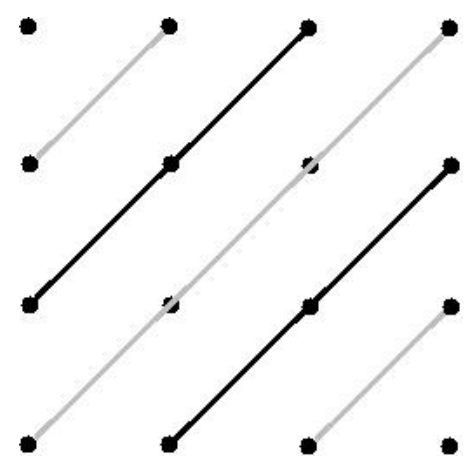

(a) $\Omega_{r_{\beta}^{+}, 0}$

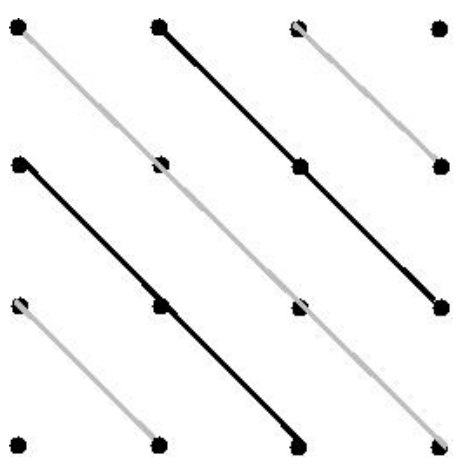

(b) $\Omega_{0, r_{\beta}^{+}}$

Figura 5.4: Ilustração dos dois possíveis estados de equilíbrio com ondas de densidade no caso $d$-wave. Se $\lambda \rightarrow \frac{\gamma}{2}$, o cor cinza representaria as franjas com densidade media eletrônica por site igual zero (ou dois dependendo do valor de $\mu$ ) e o cor preto representaria as franjas com densidade media igual um.

Na figura acima observamos o tipo de ondas de densidade presentadas para os dois possíveis estados de equilíbrio para o caso $d$-wave. Para o caso $p$-wave notamos que o analise é idêntico mas a família de estados de equilíbrio com ondas de densidade e muito maior ${ }^{5}$. Por exemplo, no caso $p$-wave podem existir ondas de densidade com franjas horizontais ou verticais, a diferença do caso $d$-wave onde este tipo de solução não é permitida no limite de baixas temperaturas.

\footnotetext{
${ }^{5} \mathrm{O}$ número de configurações possíveis para parâmetros $\mu, \lambda$ e $\gamma$ fixos seria $N=2^{8}-2=254$.
} 


\section{Outra Condição para a Existência de Ondas de Densidade}

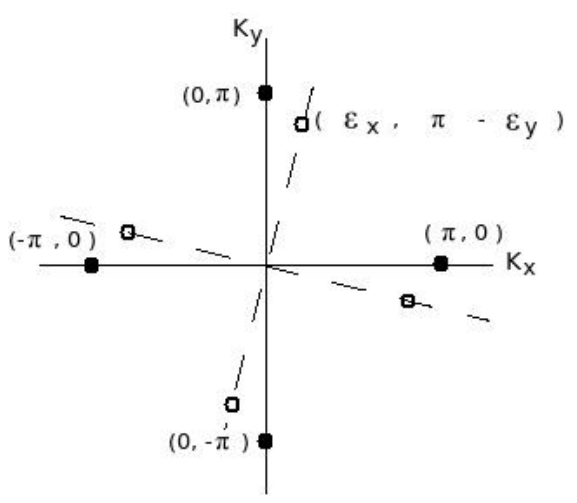

- Hamiltoniano aproximado de campo médio sera Figura 5.5: Ilustração do invariante por permutação de blocos "infinitos". deslocamento dos máximos da função de acoplamento $\hat{v}\left(K_{x}, K_{y}\right)$.

Consideraremos agora este caso $\left(\epsilon_{x}, \epsilon_{y}<<1\right)$ em que os máximos da função de acoplamento da interação bipolaron-elétron estão um pouco deslocados dos pontos $( \pm \pi, 0),(0, \pm \pi)$. Seguindo os mesmos passos feitos anteriormente calculamos a pressão termodinâmica (5.8), assim

$p_{\Lambda}\left(\mu, U_{0}, \hat{w}, \beta\right)=\frac{\ln 2}{\beta}+\mu+\sup _{\mathfrak{b}_{1}, \mathfrak{b}_{2}, \mathfrak{b}_{3}, \mathfrak{b}_{4}}\left(-\hat{w}|\mathfrak{b}|^{2}+\frac{1}{\beta|\Lambda|} \sum_{(x, y) \in \Lambda} \ln \left(1+\mathrm{e}^{-\frac{\beta U_{0}}{2}} \cosh (\beta g(x, y))\right)\right)$

onde $\Lambda \subset \mathbb{Z}^{2}$ é a região ocupada por um bloco base $(|\Lambda| \rightarrow \infty),|\mathfrak{b}|^{2}=\sum_{m}\left|\mathfrak{b}_{m}\right|^{2}$ e

$$
g(x, y)=\sqrt{\varsigma^{2}+\hat{w}^{2}|\eta(x, y)|^{2}}
$$

com

$$
\eta(x, y)=\mathfrak{b}_{1}^{*}(-1)^{y} \mathrm{e}^{i\left(\epsilon_{x} x-\epsilon_{y} y\right)}+\mathfrak{b}_{2}^{*}(-1)^{x} \mathrm{e}^{-i\left(\epsilon_{y} x+\epsilon_{x} y\right)}+\mathfrak{b}_{3}^{*}(-1)^{y} \mathrm{e}^{-i\left(\epsilon_{x} x-\epsilon_{y} y\right)}+\mathfrak{b}_{4}^{*}(-1)^{x} \mathrm{e}^{i\left(\epsilon_{y} x+\epsilon_{x} y\right)}
$$


Observamos que

$$
\begin{aligned}
& \frac{1}{\beta|\Lambda|} \sum_{(x, y) \in \Lambda} \ln \left(1+\mathrm{e}^{-\frac{\beta U_{0}}{2}} \cosh (\beta g(x, y))\right)= \\
& \frac{1}{\beta|\Lambda|} \sum_{(x, y) \in\left\{(2 n+m, m) \mid(n, m) \in \mathbb{Z}^{2}\right\} \subset \Lambda} \ln \left(1+\mathrm{e}^{-\frac{\beta U_{0}}{2}} \cosh \left(\beta g_{+}(x, y)\right)\right) \\
& \quad+\frac{1}{\beta|\Lambda|} \sum_{(x, y) \in\left\{(2 n+m+1, m) \mid(n, m) \in \mathbb{Z}^{2}\right\} \subset \Lambda} \ln \left(1+\mathrm{e}^{-\frac{\beta U_{0}}{2}} \cosh \left(\beta g_{-}(x, y)\right)\right)
\end{aligned}
$$

onde

$$
g_{+}\left(x^{\prime}, y^{\prime}\right)=\sqrt{\varsigma^{2}+\hat{w}^{2}\left|\mathfrak{b}_{1}^{*} \mathrm{e}^{i\left(\epsilon_{x} x-\epsilon_{y} y\right)}+\mathfrak{b}_{2}^{*} \mathrm{e}^{-i\left(\epsilon_{y} x+\epsilon_{x} y\right)}+\mathfrak{b}_{3}^{*} \mathrm{e}^{-i\left(\epsilon_{x} x-\epsilon_{y} y\right)}+\mathfrak{b}_{4}^{*} \mathrm{e}^{i\left(\epsilon_{y} x+\epsilon_{x} y\right)}\right|^{2}}
$$

e

$$
g_{-}\left(x^{\prime}, y^{\prime}\right)=\sqrt{\varsigma^{2}+\hat{w}^{2}\left|\mathfrak{b}_{1}^{*} \mathrm{e}^{i\left(\epsilon_{x} x-\epsilon_{y} y\right)}-\mathfrak{b}_{2}^{*} \mathrm{e}^{-i\left(\epsilon_{y} x+\epsilon_{x} y\right)}+\mathfrak{b}_{3}^{*} \mathrm{e}^{-i\left(\epsilon_{x} x-\epsilon_{y} y\right)}-\mathfrak{b}_{4}^{*} \mathrm{e}^{i\left(\epsilon_{y} x+\epsilon_{x} y\right)}\right|^{2}} .
$$

Agora notamos que se $\epsilon_{x}, \epsilon_{y} \rightarrow \epsilon \rightarrow 0$

$$
\begin{aligned}
& \frac{1}{\beta|\Lambda|} \sum_{(x, y) \in \Lambda} \ln \left(1+\mathrm{e}^{-\frac{\beta U_{0}}{2}} \cosh (\beta g(x, y))\right) \\
&=\lim _{\epsilon \rightarrow 0} \frac{1}{\beta|\Lambda| \epsilon^{2}} \int_{-\epsilon L}^{\epsilon L} d y^{\prime} \int_{-\epsilon L / 2}^{\epsilon L / 2} d x^{\prime}\left(F_{+}\left(x^{\prime}, y^{\prime}\right)+F_{-}\left(x^{\prime}, y^{\prime}\right)\right) \\
&=\frac{1}{2 \beta \pi^{2}} \int_{-\pi / 2}^{\pi / 2} \int_{-\pi / 2}^{\pi / 2}\left(F_{+}\left(x^{\prime}, y^{\prime}\right)+F_{-}\left(x^{\prime}, y^{\prime}\right)\right) d x^{\prime} d y^{\prime}
\end{aligned}
$$

onde $|\Lambda|=4 L^{2},\left(x^{\prime}, y^{\prime}\right) \in \mathbb{R}^{2}$, e

$$
F_{ \pm}\left(x^{\prime}, y^{\prime}\right)=\ln \left(1+\mathrm{e}^{-\frac{\beta U_{0}}{2}} \cosh \left(\beta \tilde{g}_{ \pm}\left(x^{\prime}, y^{\prime}\right)\right)\right)
$$

com

$$
\begin{gathered}
\tilde{g}_{+}\left(x^{\prime}, y^{\prime}\right)=\sqrt{\varsigma^{2}+\hat{w}^{2}\left|\mathfrak{b}_{1}^{*} \mathrm{e}^{2 i x^{\prime}}+\mathfrak{b}_{2}^{*} \mathrm{e}^{-2 i\left(x^{\prime}+y^{\prime}\right)}+\mathfrak{b}_{3}^{*} \mathrm{e}^{-2 i x^{\prime}}+\mathfrak{b}_{4}^{*} \mathrm{e}^{2 i\left(x^{\prime}+y^{\prime}\right)}\right|^{2}} \\
\tilde{g}_{-}\left(x^{\prime}, y^{\prime}\right)=\sqrt{\varsigma^{2}+\hat{w}^{2}\left|\mathfrak{b}_{1}^{*} \mathrm{e}^{2 i\left(x^{\prime}+\epsilon / 2\right)}-\mathfrak{b}_{2}^{*} \mathrm{e}^{-2 i\left(x^{\prime}+y^{\prime}+\epsilon / 2\right)}+\mathfrak{b}_{3}^{*} \mathrm{e}^{-2 i\left(x^{\prime}+\epsilon / 2\right)}-\mathfrak{b}_{4}^{*} \mathrm{e}^{2 i\left(x^{\prime}+y^{\prime}+\epsilon / 2\right)}\right|^{2}} .
\end{gathered}
$$


Como vimos na anteriormente a densidade eletrônica por site vem dada por

$$
\Omega^{i}\left(n_{i, \uparrow}+n_{i, \downarrow}\right)=\frac{1}{\beta} \partial_{\mu} \ln \operatorname{Tr}\left(\mathrm{e}^{-\beta H_{i}}\right)
$$

onde $H_{i}$ é o Hamiltoniano local aproximado de Bogoliubov. Em nosso caso, temos agora

$$
H_{i=(x, y) \in \mathbb{Z}^{2}}=-\mu \sum_{s \in\{\uparrow, \downarrow\}} a_{i, s}^{\dagger} a_{i, s}+U_{0} n_{i, \uparrow} n_{i, \downarrow}-\hat{w}\left(\eta(x, y)^{*} c_{i}^{\dagger}+\eta(x, y) c_{i}\right)
$$

Notamos que a densidade eletrônica necessariamente varia na rede se o tamanho do bloco base tende a infinito e $\epsilon_{x}, \epsilon_{y}=\epsilon \rightarrow 0$, salvo o caso em que as constantes de Bogoliubov $\left(\mathfrak{b}_{1}, \mathfrak{b}_{2}, \mathfrak{b}_{3}, \mathfrak{b}_{4}\right)$ sejam todas idênticas a zero. Esto pode verse de (5.29) ao considerar-se uma expansão ate $\mathcal{O}\left(\epsilon^{2}\right)$, vemos que

$$
|\eta(x, y)|=\left\{\begin{array}{l}
\left|\eta_{+}(x, y)\right| \text { se } \quad(x, y) \in\left\{(2 n+m, m) \mid(n, m) \in \mathbb{Z}^{2}\right\} \subset \Lambda \\
\left|\eta_{-}(x, y)\right| \text { se }(x, y) \in\left\{(2 n+m+1, m) \mid(n, m) \in \mathbb{Z}^{2}\right\} \subset \Lambda
\end{array}\right.
$$

onde

$$
\eta_{+}(x, y)=\mathfrak{b}_{1}^{*} \mathrm{e}^{i\left(\epsilon_{x} x-\epsilon_{y} y\right)}+\mathfrak{b}_{2}^{*} \mathrm{e}^{-i\left(\epsilon_{y} x+\epsilon_{x} y\right)}+\mathfrak{b}_{3}^{*} \mathrm{e}^{-i\left(\epsilon_{x} x-\epsilon_{y} y\right)}+\mathfrak{b}_{4}^{*} \mathrm{e}^{i\left(\epsilon_{y} x+\epsilon_{x} y\right)}
$$

e

$$
\eta_{-}(x, y)=\mathfrak{b}_{1}^{*} \mathrm{e}^{i\left(\epsilon_{x} x-\epsilon_{y} y\right)}-\mathfrak{b}_{2}^{*} \mathrm{e}^{-i\left(\epsilon_{y} x+\epsilon_{x} y\right)}+\mathfrak{b}_{3}^{*} \mathrm{e}^{-i\left(\epsilon_{x} x-\epsilon_{y} y\right)}-\mathfrak{b}_{4}^{*} \mathrm{e}^{i\left(\epsilon_{y} x+\epsilon_{x} y\right)}
$$

Observamos então para $\epsilon_{x}, \epsilon_{y}=\epsilon \rightarrow 0$ que

$$
\begin{aligned}
\eta_{+}(x, y)=\left(\mathfrak{b}_{1}^{*}+\mathfrak{b}_{2}^{*}+\right. & \left.\mathfrak{b}_{3}^{*}+\mathfrak{b}_{4}^{*}\right) \\
& \begin{aligned}
+i \epsilon\left((x+y)\left(\mathfrak{b}_{4}^{*}-\mathfrak{b}_{2}^{*}\right)+(x-y)\left(\mathfrak{b}_{1}^{*}-\mathfrak{b}_{3}^{*}\right)\right) \\
\quad-\epsilon^{2}\left((x-y)^{2}\left(\mathfrak{b}_{1}^{*}+\mathfrak{b}_{3}^{*}\right)+(x+y)^{2}\left(\mathfrak{b}_{4}^{*}+\mathfrak{b}_{2}^{*}\right)\right)+\mathcal{O}\left(\epsilon^{3}\right)
\end{aligned}
\end{aligned}
$$




$$
\begin{aligned}
\eta_{-}(x, y)=\left(\mathfrak{b}_{1}^{*}-\mathfrak{b}_{2}^{*}+\right. & \left.\mathfrak{b}_{3}^{*}-\mathfrak{b}_{4}^{*}\right) \\
& \begin{aligned}
+i \epsilon\left((x+y)\left(\mathfrak{b}_{2}^{*}-\mathfrak{b}_{4}^{*}\right)+(x-y)\left(\mathfrak{b}_{1}^{*}-\mathfrak{b}_{3}^{*}\right)\right) \\
\quad-\epsilon^{2}\left((x-y)^{2}\left(\mathfrak{b}_{1}^{*}+\mathfrak{b}_{3}^{*}\right)-(x+y)^{2}\left(\mathfrak{b}_{4}^{*}+\mathfrak{b}_{2}^{*}\right)\right)+\mathcal{O}\left(\epsilon^{3}\right)
\end{aligned}
\end{aligned}
$$

assim se $\mathfrak{b}_{1}=\mathfrak{b}_{3}$ e $\mathfrak{b}_{2}=\mathfrak{b}_{4}$ podem existir constantes de Bogoliubov que anulem a variação de $\eta(x, y)$ só ate $\mathcal{O}(\epsilon)$. Daqui pode observar-se que a única possibilidade para que $|\eta(x, y)|$ seja constante é que $\mathfrak{b}_{1}=\mathfrak{b}_{2}=\mathfrak{b}_{3}=\mathfrak{b}_{4}=0$.

Mostraremos agora que em geral a solução "zero" para as constantes de Bogoliubov não são maximizantes da pressão termodinâmica (5.28), e que tem uma região de parâmetros $\left(\mu, U_{0}, \hat{w}\right)$ onde existe uma terna $\left(\mathfrak{b}_{1}, \mathfrak{b}_{2}, \mathfrak{b}_{3}, \mathfrak{b}_{4}\right)$ diferente de zero que maximiza a pressão termodinâmica, e por tanto neste caso $(\epsilon \rightarrow 0)$ podem se apresentar ondas de densidade eletrônica na rede com uma única terna de constantes de Bogoliubov maximizantes, a diferença dos casos discutido anteriormente ( $d, p$-wave).

Definimos agora a função

$$
\mathfrak{G}\left(\mathfrak{b}_{1}, \mathfrak{b}_{2}, \mathfrak{b}_{3}, \mathfrak{b}_{4}\right):=-\hat{w}|\mathfrak{b}|^{2}+\frac{1}{2 \beta \pi^{2}} \int_{-\pi / 2}^{\pi / 2} \int_{-\pi / 2}^{\pi / 2}\left(F_{+}\left(x^{\prime}, y^{\prime}\right)+F_{-}\left(x^{\prime}, y^{\prime}\right)\right) d x^{\prime} d y^{\prime}
$$

assim temos que para $\epsilon_{x}, \epsilon_{y} \rightarrow \epsilon \rightarrow 0$

$$
p_{\Lambda}\left(\mu, U_{0}, \hat{w}, \beta\right) \rightarrow \frac{\ln 2}{\beta}+\mu+\sup _{\mathfrak{b}_{1}, \mathfrak{b}_{2}, \mathfrak{b}_{3}, \mathfrak{b}_{4}} \mathfrak{G}\left(\mathfrak{b}_{1}, \mathfrak{b}_{2}, \mathfrak{b}_{3}, \mathfrak{b}_{4}\right)
$$

Observamos que

$$
\mathfrak{G}(0,0,0,0)=\frac{1}{\beta} \ln \left(1+\mathrm{e}^{-\beta \lambda} \cosh (\beta|\mu-\lambda|)\right)
$$

onde $\lambda=\frac{U_{0}}{2}$. Ao considerar um deslocamento $\overrightarrow{\delta \mathfrak{b}}=(\delta \mathfrak{b}, \delta \mathfrak{b}, \delta \mathfrak{b}, \delta \mathfrak{b})$ a partir do ponto zero 
notamos que

$$
\begin{gathered}
\mathfrak{G}(\overrightarrow{\delta \mathfrak{b}})-\mathfrak{G}(\overrightarrow{0})=-4 \hat{w}|\delta \mathfrak{b}|^{2}+\frac{\tanh (\beta|\mu-\lambda|)}{1+\mathrm{e}^{\lambda \beta} \operatorname{sech}(\beta|\mu-\lambda|)} \cdot \frac{1}{2|\mu-\lambda|} \frac{2 \hat{w}^{2}|\delta \mathfrak{b}|^{2}}{\pi^{2}} \\
\cdot \int_{-\pi / 2}^{\pi / 2} \int_{-\pi / 2}^{\pi / 2}\left[\cos ^{2}\left(2\left(x^{\prime}+y^{\prime}\right)\right)+\cos ^{2}\left(2 x^{\prime}\right)+\right. \\
\left.\cos ^{2}\left(2\left(x^{\prime}+y^{\prime}+\epsilon / 2\right)\right)+\cos ^{2}\left(2\left(x^{\prime}+\epsilon / 2\right)\right)\right] d x^{\prime} d y^{\prime}
\end{gathered}
$$$$
+\mathcal{O}\left(|\delta \mathfrak{b}|^{4}\right)
$$

$\mathrm{Ou}$

$$
\mathfrak{G}(\overrightarrow{\delta \mathfrak{b}})-\mathfrak{G}(\overrightarrow{0})=-4 \hat{w}|\delta \mathfrak{b}|^{2}+\frac{\tanh (\beta|\mu-\lambda|)}{1+\mathrm{e}^{\lambda \beta} \operatorname{sech}(\beta|\mu-\lambda|)} \cdot \frac{2}{|\mu-\lambda|} \hat{w}^{2}|\delta \mathfrak{b}|^{2}+\mathcal{O}\left(|\delta \mathfrak{b}|^{4}\right)
$$

Como observamos antes no limite de baixas temperaturas $(\beta>>1)$ temos que

$$
\frac{\tanh (\beta|\mu-\lambda|)}{1+\mathrm{e}^{\lambda \beta} \operatorname{sech}(\beta|\mu-\lambda|)} \rightarrow H(|\mu-\lambda|-\lambda)
$$

assim no limite de baixas temperaturas

$$
\mathfrak{G}(\overrightarrow{\delta \mathfrak{b}})-\mathfrak{G}(\overrightarrow{0})>0
$$

se

$$
H(|\mu-\lambda|-\lambda)>\frac{2|\mu-\lambda|}{\hat{w}}
$$

Dado que $\hat{w}>0$, se $\lambda<|\mu-\lambda|<\hat{w} / 2$ temos então que $\mathfrak{G}(\overrightarrow{\delta \mathfrak{b}})-\mathfrak{G}(\overrightarrow{0})>0$, no limite de baixas temperaturas. Esto demostra que existe uma região de parâmetros onde temos uma terna de constantes de Bogoliubov diferentes da solução "zero" que maximizam a energia livre. 
Tal como é feito em (5.27), temos a densidade eletrônica por site $\delta$ dada por

$$
\delta(x, y)=1+\frac{(\mu-\lambda)}{\sqrt{(\mu-\lambda)^{2}+\hat{w}^{2}|\eta(x, y)|^{2}}} H(|\mu-\lambda|-\lambda) .
$$

Na figura abaixo colocamos o gráfico da função $\delta(x, y)$ no caso em que todas as constantes de Bogoliubov são iguais, só para exemplificar a estrutura da função.

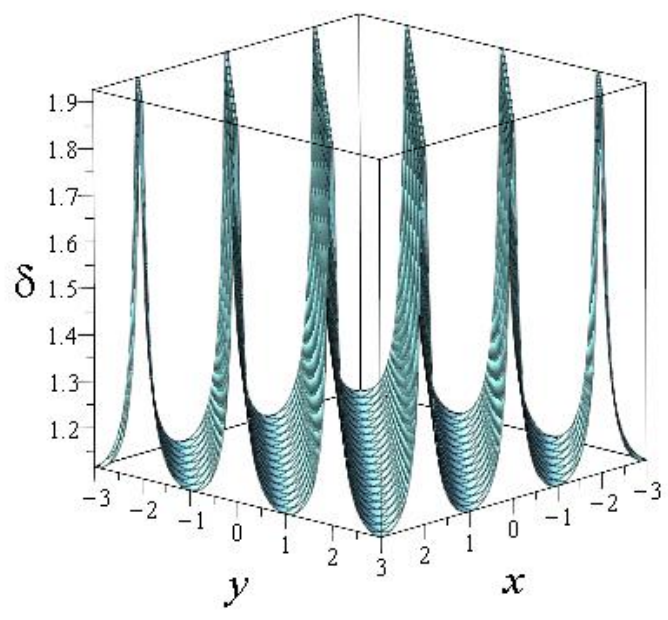

Figura 5.6: Gráfico da função $\delta(x, y)$ para $\lambda=0.5, \mu=1.2$ e $\hat{w}=1.5$. A escolha dos parâmetros satisfaz a condição $\lambda<|\mu-\lambda|<\hat{w} / 2$, e se tomo o limite $\beta>>1$. 


\section{Efeito do Termo Cinético na Pressão Termodinâmica}

Nesta secção mostraremos que ao perturbar o Hamiltoniano aproximado de Bogoliubov com o termo cinético não se modifica a "estrutura" da pressão termodinâmica de equilíbrio e portanto a presença das ondas de densidade nos possíveis estados de equilíbrio. Como se mostro anteriormente o regime de "strong coupling" pode aproximar-se a um Hamiltoniano que é uma soma de operadores locais, ao considerar o termo cinético pode verse que para um parâmetro de hopping $\epsilon$ pequeno a pressão se deslocara pouco da pressão "local".

A diferença do Hamiltoniano aproximado de Bogoliubov

$$
\begin{aligned}
H_{s c}\left(\mathfrak{b}_{1}, \mathfrak{b}_{2}, \mathfrak{b}_{3}, \mathfrak{b}_{4}\right) \doteq-\mu & \sum_{s \in\{\uparrow, \downarrow\}, x \in \Lambda} n_{x, s}+U_{0} \sum_{x \in \Lambda} n_{x, \uparrow} n_{x, \downarrow} \\
& -\hat{w} \sum_{m=1}^{4} \sum_{x \in \Lambda}\left(\left(\mathfrak{b}_{m}^{*} \mathrm{e}^{i k_{m} \cdot x} a_{x, \uparrow} a_{x, \downarrow}\right)^{\dagger}+\mathfrak{b}_{m}^{*} \mathrm{e}^{i k_{m} \cdot x} a_{x, \uparrow} a_{x, \downarrow}\right)
\end{aligned}
$$

que é uma soma de operadores locais que comutam, o termo cinético $\mathbf{T}_{f}$

$$
\mathbf{T}_{f}=\sum_{s \in\{\uparrow, \downarrow\}, x \in \Lambda,|z|=1} a_{x+z, s}^{\dagger} a_{x, s}
$$

não apresenta esta simetria. Notamos entanto que o Hamiltoniano

$$
\widetilde{H}=\epsilon \mathbf{T}_{f}+H_{s c}\left(\mathfrak{b}_{1}, \mathfrak{b}_{2}, \mathfrak{b}_{3}, \mathfrak{b}_{4}\right)
$$

é invariante por traslação de "blocos".

Notamos que as constantes de Bogoliubov que determinam a pressão de equilíbrio não vão diferir muito das "novas constantes" se se perturba o Hamiltoniano aproximado com um termo cinético com hopping $\epsilon \rightarrow 0$. Esto pode observar-se da desigualdade de Bogoliubov

$$
\left|\ln \operatorname{Tr}\left(\mathrm{e}^{A+B}\right)-\ln \operatorname{Tr}\left(\mathrm{e}^{A}\right)\right| \leq\|B\| .
$$


Temos então similar a (5.8) que a nova pressão de equilíbrio vem dada por

$$
p_{\Lambda}\left(\mu, U_{0}, \hat{w}, \epsilon, \beta\right)=\sup _{\mathfrak{b}_{1}, \mathfrak{b}_{2}, \mathfrak{b}_{3}, \mathfrak{b}_{4}}\left(-\hat{w}|\mathfrak{b}|^{2}+\tilde{p}_{\Lambda}\left(\mathfrak{b}_{1}, \mathfrak{b}_{2}, \mathfrak{b}_{3}, \mathfrak{b}_{4}\right)\right)
$$

onde

$$
\tilde{p}_{\Lambda}\left(\mu, U_{0}, \hat{w}, \epsilon, \beta\right) \doteq \frac{1}{\beta|\Lambda|} \ln \operatorname{Tr}\left(\mathrm{e}^{-\beta\left(\epsilon \mathbf{T}_{f}+H_{s c}\right)}\right)
$$

usando a desigualdade de Bogoliubov temos que

$$
\left\|\tilde{p}_{\Lambda}\left(\mu, U_{0}, \hat{w}, \epsilon, \beta\right)-p\left(\mathfrak{b}_{1}, \mathfrak{b}_{2}, \mathfrak{b}_{3}, \mathfrak{b}_{4}\right)\right\| \leq \epsilon \frac{\left\|\mathbf{T}_{f}\right\|}{|\Lambda|}
$$

onde lembramos que

$$
p\left(\mathfrak{b}_{1}, \mathfrak{b}_{2}, \mathfrak{b}_{3}, \mathfrak{b}_{4}\right):=\frac{1}{\beta|\Lambda|} \ln \operatorname{Tr}\left(\mathrm{e}^{-\beta H_{s c}\left(\mathfrak{b}_{1}, \mathfrak{b}_{2}, \mathfrak{b}_{3}, \mathfrak{b}_{4}\right)}\right)
$$

assim

$$
p_{\Lambda}\left(\mu, U_{0}, \hat{w}, \epsilon, \beta\right)=\sup _{\mathfrak{b}_{1}, \mathfrak{b}_{2}, \mathfrak{b}_{3}, \mathfrak{b}_{4}}(\underbrace{-\hat{w}|\mathfrak{b}|^{2}+p\left(\mathfrak{b}_{1}, \mathfrak{b}_{2}, \mathfrak{b}_{3}, \mathfrak{b}_{4}\right)}_{\mathfrak{g}(\overrightarrow{\mathfrak{b}})}+\mathcal{O}_{\overrightarrow{\mathfrak{b}}}(\epsilon))
$$

onde $\overrightarrow{\mathfrak{b}}=\left(\mathfrak{b}_{1}, \mathfrak{b}_{2}, \mathfrak{b}_{3}, \mathfrak{b}_{4}\right)$

Seja

$$
\sup _{\overrightarrow{\mathfrak{b}}} \mathfrak{g}(\overrightarrow{\mathfrak{b}})=\mathfrak{g}\left(\overrightarrow{\mathfrak{b}_{\mathfrak{o}}}\right)
$$

temos que

$$
\sup _{\overrightarrow{\mathfrak{b}}}\left(\mathfrak{g}(\overrightarrow{\mathfrak{b}})+\mathcal{O}_{\overrightarrow{\mathfrak{b}}}(\epsilon)\right)=\mathfrak{g}\left(\overrightarrow{\mathfrak{b}^{\prime}}\right)+\mathcal{O}_{\overrightarrow{\mathfrak{b}^{\prime}}}(\epsilon) \leq \sup _{\overrightarrow{\mathfrak{b}}} \mathfrak{g}(\overrightarrow{\mathfrak{b}})+\mathcal{O}_{\overrightarrow{\mathfrak{b}}}(\epsilon)=\mathfrak{g}\left(\overrightarrow{\mathfrak{b}_{\mathfrak{o}}}\right)+\mathcal{O}_{\overrightarrow{\mathfrak{b}}}(\epsilon)
$$

onde

$$
\sup _{\overrightarrow{\mathfrak{b}}} \mathcal{O}_{\overrightarrow{\mathfrak{b}}}(\epsilon)=\mathcal{O}_{\overrightarrow{\mathfrak{b}}}(\epsilon)
$$

assim

$$
\mathfrak{g}\left(\overrightarrow{\mathfrak{b}^{\prime}}\right)-\mathfrak{g}\left(\overrightarrow{\mathfrak{b}_{\mathcal{O}}}\right) \leq \mathcal{O}_{\overrightarrow{\mathfrak{b}}}(\epsilon)-\mathcal{O}_{\overrightarrow{\mathfrak{b}^{\prime}}}(\epsilon)=\mathcal{O}(\epsilon)
$$


se $\epsilon \rightarrow 0$

$$
\left|\mathfrak{g}\left(\overrightarrow{\mathfrak{b}^{\prime}}\right)-\mathfrak{g}\left(\overrightarrow{\mathfrak{b}_{\mathcal{o}}}\right)\right| \rightarrow 0
$$

$\mathrm{Ou}$

$$
\mathfrak{g}\left(\overrightarrow{\mathfrak{b}^{\prime}}\right)=\mathfrak{g}\left(\overrightarrow{\mathfrak{b}_{\mathfrak{o}}}\right)+\delta \mathfrak{g}_{\epsilon}
$$

como $\sup _{\overrightarrow{\mathfrak{b}}} \mathfrak{g}(\overrightarrow{\mathfrak{b}})=\mathfrak{g}\left(\overrightarrow{\mathfrak{b}_{\mathfrak{o}}}\right)$ corresponde a um único ponto $\overrightarrow{\mathfrak{b}_{0}}$ (considerando por exemplo o caso tratado na secção anterior), temos que (5.32) implica que $\left|\overrightarrow{\mathfrak{b}^{\prime}}-\overrightarrow{\mathfrak{b}_{\mathfrak{o}}}\right| \rightarrow 0$, pelo que a correção com $\epsilon \rightarrow 0$ não perturbara muito a posição das constantes $\left(\mathfrak{b}_{1}, \mathfrak{b}_{2}, \mathfrak{b}_{3}, \mathfrak{b}_{4}\right)$ maximizantes.

Nosso interesse agora é estudar o efeito de o termo cinético com um hopping pequeno $\epsilon \rightarrow 0$ sobre as ondas de densidade nos estados de equilíbrio com $\epsilon=0$. O cálculo desta pressão é mais difícil mas em geral pode ser usada uma expansão conhecida como "expansão por clusters".

Tal como é exposto em [33], apresentamos aqui uma versão geral do teorema de expansão por clusters. Este teorema nos permite obter, para alguns modelos, uma serie infinta convergente para a pressão termodinâmica em um domínio de parâmetros. Seja $(\mathbb{A}, \mathcal{A}, \mu)$ um espaço de medida, $\mu$ é finita. A função de partição é definida como

$$
\mathcal{Z}=\sum_{n=0}^{\infty} \frac{1}{n !} \int d \mu\left(A_{1}\right) \cdots \int d \mu\left(A_{n}\right) \prod_{1 \leq i<j \leq n}\left(1+\zeta\left(A_{i}, A_{j}\right)\right)
$$

onde $\zeta\left(A_{i}, A_{j}\right)$ é uma medida simétrica que atua sobre $\mathbb{A} \times \mathbb{A}$.

Teorema 2. Se $\left|1+\zeta\left(A, A^{\prime}\right)\right| \leq 1$ para todo $A, A^{\prime} \in \mathbb{A}$ e a $(A)$ uma função não negativa tal que para todo $A \in \mathbb{A}$

$$
\int d|\mu|\left(A^{\prime}\right)\left|\zeta\left(A, A^{\prime}\right)\right| e^{a\left(A^{\prime}\right)} \leq a(A)
$$

$e \int d|\mu|(A) e^{a(A)}<\infty$, então

$$
\ln (\mathcal{Z})=\sum_{n=1}^{\infty} \int d \mu\left(A_{1}\right) \cdots \int d \mu\left(A_{n}\right) \varphi\left(A_{1}, \cdots, A_{n}\right)
$$


a função $\varphi\left(A_{1}, \cdots, A_{n}\right)$ é definida como

$$
\varphi\left(A_{1}, \cdots, A_{n}\right)=\left\{\begin{array}{cl}
1 & \text { se } n=1 \\
\frac{1}{n !} \sum_{G \in \mathcal{C}_{n}} \prod_{(i, j) \in G} \zeta\left(A_{i}, A_{j}\right) & \text { se } n \geq 2
\end{array}\right.
$$

onde $\mathcal{C}_{n}$ é o conjunto dos grafos conetados de $n$ vértices.

Em geral para uma situação na rede $\mathbb{Z}^{2}$ temos um Hamiltoniano

$$
H_{\Lambda}^{\phi}=\sum_{A \subset \Lambda} \phi(A)
$$

onde $\phi(A)$ é a interação restringida no setor $A \subset \Lambda$. A função de partição no volume $\Lambda$, dependendo do modelo em particular, pode escrever-se como uma soma de produtos de "pesos" que atuam sobre uma família de objetos chamados "polímeros" [32].

$$
\mathcal{Z}(\Lambda)=\operatorname{Tr}\left(\mathrm{e}^{-\beta H_{\Lambda}^{\phi}}\right)=\sum \prod_{\mathcal{A} \in \Xi} \Phi(\mathcal{A})
$$

A soma é sobre as famílias de polímeros $(\Xi)$, e $\mathcal{A}$ denota um polímero pertencente a uma das famílias.

Tendo uma visão geral da expansão por clusters da pressão termodinâmica para um sistema qualquer (que admita expansão por clusters) voltamos agora a nosso problema. Para fazer uma expansão da função de partição de nosso sistema usamos a formula de Duhamel tal como é feito em [34]. Usando a formula de Duhamel obtemos uma expressão da função de partição do tipo "expansão por clusters".

Seja um Hamiltoniano (ou um operador geral $H$ )

$$
H=H_{0}+H^{\prime}
$$

notamos que

$$
\partial_{\beta}\left(\mathrm{e}^{\beta H_{0}} \mathrm{e}^{-\beta H}\right)=-\mathrm{e}^{\beta H_{0}} H^{\prime} \mathrm{e}^{-\beta H}
$$


assim integrando temos que

$$
\mathrm{e}^{-\beta H}=\mathrm{e}^{-\beta H_{0}}-\int_{0}^{\beta} \mathrm{e}^{-\left(\beta-\beta_{1}\right) H_{0}} H^{\prime} \mathrm{e}^{-\beta_{1} H} d \beta_{1}
$$

e de forma recursiva obtemos a formula de Duhamel

$\mathrm{e}^{-\beta H}=\mathrm{e}^{-\beta H_{0}}+\sum_{n=1}^{\infty}(-1)^{n} \int_{0}^{\beta} d \beta_{1} \int_{0}^{\beta_{1}} d \beta_{2} \cdots \int_{0}^{\beta_{n-1}} d \beta_{n} \mathrm{e}^{-\left(\beta-\beta_{1}\right) H_{0}} H^{\prime} \mathrm{e}^{-\left(\beta_{1}-\beta_{2}\right) H_{0}} H^{\prime} \cdots H^{\prime} \mathrm{e}^{-\beta_{n} H_{0}}$.

Agora consideraremos para nosso caso $H_{0}=H_{s c}\left(\mathfrak{b}_{1}, \mathfrak{b}_{2}, \mathfrak{b}_{3}, \mathfrak{b}_{4}\right)$ e $H^{\prime}=\epsilon \mathbf{T}_{f}$,

$$
\mathbf{T}_{f}=\sum_{A \subset \Lambda} T_{A}
$$

onde

$$
T_{A}=\left\{\begin{array}{cll}
a_{x, \uparrow}^{\dagger} a_{y, \uparrow}+a_{y, \uparrow}^{\dagger} a_{x, \uparrow}+a_{x, \downarrow}^{\dagger} a_{y, \downarrow}+a_{y, \downarrow}^{\dagger} a_{x, \downarrow} & \text { se } & |x-y|=1 \\
0 & \text { se } & |x-y| \neq 1
\end{array}\right.
$$

assim

$$
\begin{aligned}
& \mathcal{Z}=\operatorname{Tr}\left(\mathrm{e}^{-\beta H}\right)=\operatorname{Tr}\left(\mathrm{e}^{-\beta H_{0}}\right)+ \\
& \sum_{n=1}^{\infty}(-\epsilon)^{n} \int_{0}^{\beta} d \beta_{1} \int_{0}^{\beta_{1}} d \beta_{2} \cdots \int_{0}^{\beta_{n-1}} d \beta_{n} \sum_{A_{1}, \cdots, A_{n}} \operatorname{Tr}\left(\mathrm{e}^{-\left(\beta-\beta_{1}\right) H_{0}} T_{A_{1}} \mathrm{e}^{-\left(\beta_{1}-\beta_{2}\right) H_{0}} T_{A_{2}} \cdots T_{A_{n}} \mathrm{e}^{-\beta_{n} H_{0}}\right) .
\end{aligned}
$$

Tal como é explicado em [34], cada conjunto de subconjuntos $\left\{A_{1}, \cdots, A_{n}\right\}$ pode ser decomposto em subconjuntos conetados $\left\{\mathcal{A}_{1}, \cdots, \mathcal{A}_{l}\right\}$ com $l \leq n$. Estos subconjuntos conetados seriam os clusters neste caso. Com esta definição é possível reescrever a função de partição (expandida com a formula de Duhamel) na forma de expansão por clusters. Dai a analiticidade da pressão termodinâmica é uma consequência direta do teorema de expansão por clusters provado em [32]. 

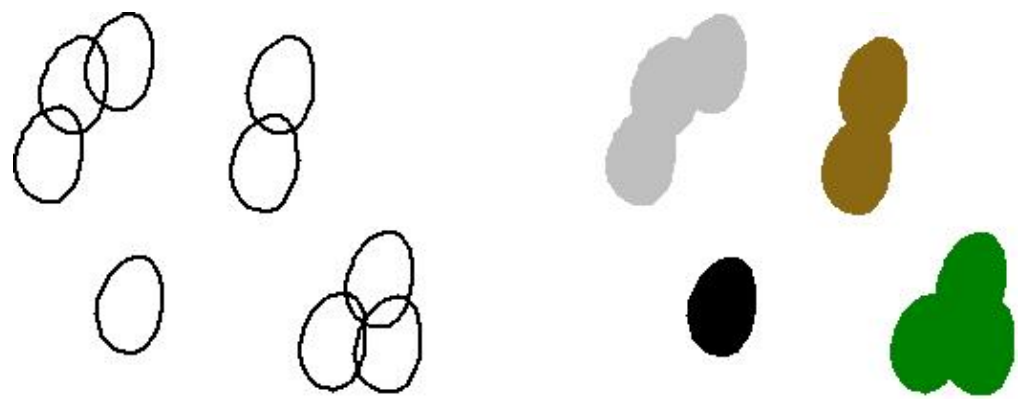

Figura 5.7: Ilustração geral dos clusters $\mathcal{A}_{i}$ (cinza, preto, verde e marrom) formados por os subconjuntos $A_{i} \subset \mathbb{Z}^{2}$.

Definimos agora mais precisamente os clusters para nosso problema. Seja $\left(A_{1}, \ldots, A_{n}\right)$ uma sequencia de $n \in \mathbb{N}$ subconjuntos de $\Lambda$. Associamos a ela o grafo (não orientado) de $n$ vértices

$$
G\left(A_{1}, \ldots, A_{n}\right)=\left\{\{i, j\}: i, j=1, . ., n, i \neq j, A_{i} \cap A_{j} \neq \emptyset\right\} .
$$

Sejam $\left\{K_{1}, . ., K_{j}\right\} \subset P(\{1, \ldots, n\}), j \leq n$, o conjunto das partes conexas deste grafo. Definimos também

$$
\mathcal{A}_{k}=\cup_{p \in K_{k}} A_{p}, k=1, \ldots, j .
$$

$\left\{\mathcal{A}_{1}, \ldots, \mathcal{A}_{j}\right\}$ é a decoposição, disjunta, do suporte $\cup_{p=1}^{n} A_{p}$ da sequência $\left(A_{1}, \ldots, A_{n}\right)$. Os subconjuntos $\mathcal{A}_{k}$ são os "clusters" dessa sequência.

Seja

$$
\mathfrak{C}_{n}: P(\Lambda)^{n} \rightarrow P(P(\Lambda))
$$

o mapa que à sequência $\left(A_{1}, \ldots, A_{n}\right)$ associa o conjunto $\left\{\mathcal{A}_{1}, \ldots, \mathcal{A}_{j}\right\}$ de seus clusters ${ }^{6}$.

Expressamos agora (5.36) em termos dos polímeros $\mathcal{A}_{i}$, tal como é feito em [34]. A soma sobre os conjuntos $A_{i}$ sera trocada numa soma sobre os polímeros $\mathcal{A}_{i}$, como cada conjunto $\left\{A_{1}, \cdots, A_{n}\right\}$ pertence a um polímero ou um conjunto de polímeros é simples

\footnotetext{
${ }^{6} \mathcal{P}(\Lambda)$ é o conjunto potencia de $\Lambda$.
} 
notar que para uma função geral $\Upsilon:\left\{A_{1}, \cdots, A_{n}\right\} \rightarrow \mathbb{R}$ temos

$$
\begin{aligned}
\sum_{A_{1}, \cdots, A_{n}} \Upsilon\left(A_{1}, \cdots, A_{n}\right)=\sum_{\mathcal{A}_{1}} \sum_{\substack{A_{1}, \cdots, A_{n}: \\
\mathfrak{C}_{n}\left(A_{1}, \cdots, A_{n}\right)=\mathcal{A}_{1}}} \Upsilon\left(A_{1}, \cdots, A_{n}\right)+ \\
\sum_{\substack{\mathcal{A}_{1}, \mathcal{A}_{2}: \\
\mathcal{A}_{1} \cap \mathcal{A}_{2}=\emptyset}} \sum_{\substack{A_{1}, \cdots, A_{n}: \\
\mathfrak{C}_{n}\left(A_{1}, \cdots, A_{k}\right)=\mathcal{A}_{1} \cup \mathcal{A}_{2}}} \Upsilon\left(A_{1}, \cdots, A_{n}\right)+\cdots
\end{aligned}
$$

Seja agora o "peso" $\rho\left(\mathcal{A}_{i}\right)$

$$
\begin{gathered}
\rho\left(\mathcal{A}_{i}\right)=\mathrm{e}^{-\beta p_{0}\left|\mathcal{A}_{i}\right|} \sum_{n=1}^{\infty}(-\epsilon)^{n} \int_{0}^{\beta} d \beta_{1} \int_{0}^{\beta_{1}} d \beta_{2} \cdots \int_{0}^{\beta_{n-1}} d \beta_{n} \\
\sum_{\substack{A_{1}, \cdots, A_{n}: \\
\mathfrak{C}_{n}\left(A_{1}, \cdots, A_{n}\right)=\mathcal{A}_{i}}} \operatorname{Tr}_{\mathcal{A}_{i}}\left(\mathrm{e}^{-\left(\beta-\beta_{1}\right) H_{0}} T_{A_{1}} \mathrm{e}^{-\left(\beta_{1}-\beta_{2}\right) H_{0}} T_{A_{2}} \cdots T_{A_{n}} \mathrm{e}^{-\beta_{n} H_{0}}\right)
\end{gathered}
$$

onde $\operatorname{Tr}_{\mathcal{A}_{i}}(\cdot)$ é o traço restringido no polímero $\mathcal{A}_{i}$. Temos então que

$$
\mathcal{Z}=\mathrm{e}^{\beta p_{0}|\Lambda|}\left(1+\sum_{l} \sum_{\substack{\mathcal{A}_{1}, \cdots, \mathcal{A}_{l}: \\ \mathcal{A}_{i} \cap \mathcal{A}_{j}=\emptyset}} \prod_{i=1}^{l} \rho\left(\mathcal{A}_{i}\right)\right)
$$

$\mathrm{ou}$

$$
\mathcal{Z}=\mathrm{e}^{\beta p_{0}|\Lambda|}\left(1+\sum_{\mathcal{A}_{1}} \rho\left(\mathcal{A}_{1}\right)+\frac{1}{2} \sum_{\mathcal{A}_{1}, \mathcal{A}_{2}} \rho\left(\mathcal{A}_{1}\right) \rho\left(\mathcal{A}_{2}\right) \tilde{\varphi}\left(\mathcal{A}_{1}, \mathcal{A}_{2}\right)+\cdots\right)
$$

onde

$$
\tilde{\varphi}\left(\mathcal{A}_{1}, \mathcal{A}_{2}\right)=\left\{\begin{array}{lll}
1 & \text { se } & \mathcal{A}_{1} \cap \mathcal{A}_{2}=\emptyset \\
0 & \text { se } & \mathcal{A}_{1} \cap \mathcal{A}_{2} \neq \emptyset .
\end{array}\right.
$$

Observamos que o termo $\rho\left(\mathcal{A}_{1}\right)$ considera o caso em que os conjuntos $\left\{A_{1}, \cdots, A_{m}\right\}$ pertencem a um só polímero $\mathcal{A}_{1}$, em geral um dado conjunto $\left\{A_{1}, \cdots, A_{m}\right\}$ terá mas de uma "componente de polímero", daqui em (5.39) estão considerados todos os casos possíveis que se apresentam na soma "original" em (5.36). Se $\mathcal{A}_{i} \cap \mathcal{A}_{j}=\emptyset \operatorname{com} i \neq j$ 
temos (como é provado para operadores fermiônicos em [31]) que

$$
\operatorname{tr}_{\mathcal{A}_{i} \cup \mathcal{A}_{j}}(\cdot)=\operatorname{tr}_{\mathcal{A}_{i}}(\cdot) \operatorname{tr}_{\mathcal{A}_{j}}(\cdot)
$$

onde $\operatorname{tr}(\cdot)$ é o traço normalizado.

Assim de (5.37) e a propriedade do traço para operadores fermiônicos que atuam em regiões disjuntas temos (5.39). De (5.40) pode observar-se a diretamente a estrutura da expansão por cluster geral (5.33). A função $\zeta\left(A_{i}, A_{j}\right)$ corresponderia neste caso a

$$
\zeta\left(A_{i}, A_{j}\right)=\left\{\begin{array}{ccc}
0 & \text { se } & A_{i} \cap A_{j}=\emptyset \\
-1 & \text { se } & A_{i} \cap A_{j} \neq \emptyset
\end{array}\right.
$$

assim notamos de (5.40)

$$
\begin{aligned}
1+\sum_{\mathcal{A}_{1}} \rho\left(\mathcal{A}_{1}\right)+\frac{1}{2} \sum_{\mathcal{A}_{1}, \mathcal{A}_{2}} \rho\left(\mathcal{A}_{1}\right) \rho\left(\mathcal{A}_{2}\right) \tilde{\varphi}\left(\mathcal{A}_{1}, \mathcal{A}_{2}\right)+\cdots & \\
& =\sum_{n=0}^{\infty} \frac{1}{n !} \sum_{\mathcal{A}_{1}, \cdots, \mathcal{A}_{n}} \prod_{i=1}^{n} \rho\left(\mathcal{A}_{i}\right) \prod_{1 \leq i<j \leq n}\left(1+\zeta\left(\mathcal{A}_{i}, \mathcal{A}_{j}\right)\right) .
\end{aligned}
$$

De o teorema de expansão por clusters (5.35) temos então para (5.40) que

$$
\frac{1}{\beta|\Lambda|} \ln (\mathcal{Z})=p_{0}+\frac{1}{\beta|\Lambda|} \sum_{n=1}^{\infty} \sum_{\mathcal{A}_{1}, \cdots, \mathcal{A}_{n}} \varphi\left(\mathcal{A}_{1}, \cdots, \mathcal{A}_{n}\right) \prod_{i=1}^{n} \rho\left(\mathcal{A}_{i}\right)
$$

Analisamos agora similar como é feito em [34], a convergência da expansão, basicamente observamos em que rango de parâmetros o peso $\rho(\mathcal{A})$ decai exponencialmente com o tamanho do polímero $|\mathcal{A}|$. Em (5.38)

$$
\operatorname{Tr}_{\mathcal{A}}=\sum_{n_{\mathcal{A}}}\left\langle n_{\mathcal{A}}\left|\mathrm{e}^{-\left(\beta-\beta_{1}\right) H_{0}} T_{A_{1}} \mathrm{e}^{-\left(\beta_{1}-\beta_{2}\right) H_{0}} T_{A_{2}} \cdots T_{A_{n}} \mathrm{e}^{-\beta_{n} H_{0}}\right| n_{\mathcal{A}}\right\rangle
$$

onde $\left|n_{\mathcal{A}}\right\rangle$ é um vetor pertencente ao espaço de Hilbert gerado pela base de configurações 
eletrônicas possíveis no volume $|\mathcal{A}|$. Se observa que

$$
\begin{aligned}
& \left|\left\langle n_{\mathcal{A}}\left|\mathrm{e}^{-\left(\beta-\beta_{1}\right) H_{0}} T_{A_{1}} \mathrm{e}^{-\left(\beta_{1}-\beta_{2}\right) H_{0}} T_{A_{2}} \cdots T_{A_{n}} \mathrm{e}^{-\beta_{n} H_{0}}\right| n_{\mathcal{A}}\right\rangle\right| \leq \\
& \left\|\mathrm{e}^{-\left(\beta-\beta_{1}\right) H_{0}} T_{A_{1}} \mathrm{e}^{-\left(\beta_{1}-\beta_{2}\right) H_{0}} T_{A_{2}} \cdots T_{A_{n}} \mathrm{e}^{-\beta_{n} H_{0}}\right\| \leq\left\|\mathrm{e}^{-\beta H_{0}}\right\| \prod_{i=1}^{n}\left\|T_{A_{i}}\right\|
\end{aligned}
$$

lembrando que em nosso caso

$$
H_{0}:=H_{s c}\left(\mathfrak{b}_{1}, \mathfrak{b}_{2}, \mathfrak{b}_{3}, \mathfrak{b}_{4}\right)
$$

como $H_{s c}\left(\mathfrak{b}_{1}, \mathfrak{b}_{2}, \mathfrak{b}_{3}, \mathfrak{b}_{4}\right)=\sum_{x} H_{x}$ é uma soma de operadores locais $H_{x}$ que comutam (5.30), temos

$$
\left\|\mathrm{e}^{-\beta H_{0}}\right\|=\left\|\mathrm{e}^{-\beta H_{x}}\right\|^{|\mathcal{A}|}=\mathrm{e}^{-\beta|\mathcal{A}| E_{0}} \leq \mathrm{e}^{\beta|\mathcal{A}| p_{0}}
$$

onde $E_{0}$ é o autovalor base de $H_{x}$ e

$$
p_{0}:=\frac{1}{\beta|\Lambda|} \ln \operatorname{Tr}\left(\mathrm{e}^{-\beta H_{0}}\right)
$$

assim temos que

$$
\left|\left\langle n_{\mathcal{A}}\left|\mathrm{e}^{-\left(\beta-\beta_{1}\right) H_{0}} T_{A_{1}} \mathrm{e}^{-\left(\beta_{1}-\beta_{2}\right) H_{0}} T_{A_{2}} \cdots T_{A_{n}} \mathrm{e}^{-\beta_{n} H_{0}}\right| n_{\mathcal{A}}\right\rangle\right| \leq \mathrm{e}^{\beta|\mathcal{A}| p_{0}} \prod_{i=1}^{n}\left\|T_{A_{i}}\right\|
$$

ou que implica que

$$
\left|\operatorname{Tr}_{\mathcal{A}}\left(\mathrm{e}^{-\left(\beta-\beta_{1}\right) H_{0}} T_{A_{1}} \mathrm{e}^{-\left(\beta_{1}-\beta_{2}\right) H_{0}} T_{A_{2}} \cdots T_{A_{n}} \mathrm{e}^{-\beta_{n} H_{0}}\right)\right| \leq 4^{|\mathcal{A}|} \mathrm{e}^{\beta|\mathcal{A}| p_{0}} \prod_{i=1}^{n}\left\|T_{A_{i}}\right\|
$$

De (5.38) e (5.42) temos que

$$
|\rho(\mathcal{A})| \leq \sum_{n=1}^{\infty}(-\epsilon)^{n} 4^{|\mathcal{A}|} \sum_{\substack{A_{1}, \cdots, A_{n}: \\ \mathfrak{C}_{n}\left(A_{1}, \cdots, A_{n}\right)=\mathcal{A}}} \prod_{i=1}^{n}\left\|T_{A_{i}}\right\| \int_{0}^{\beta} d \beta_{1} \int_{0}^{\beta_{1}} d \beta_{2} \cdots \int_{0}^{\beta_{n-1}} d \beta_{n}
$$


desde que

$$
\int_{0}^{\beta} d \beta_{1} \int_{0}^{\beta_{1}} d \beta_{2} \cdots \int_{0}^{\beta_{n-1}} d \beta_{n}=\frac{\beta^{n}}{n !}
$$

temos então

$$
|\rho(\mathcal{A})| \leq 4^{|\mathcal{A}|} \sum_{n=1}^{\infty} \frac{(\beta \epsilon)^{n}}{n !} \sum_{\substack{A_{1}, \cdots, A_{n}: \\ \mathfrak{C}_{n}\left(A_{1}, \cdots, A_{n}\right)=\mathcal{A}}} \prod_{i=1}^{n}\left\|T_{A_{i}}\right\|
$$

como

$$
\mathcal{A}=\bigcup_{i=1}^{n} A_{i} \Longrightarrow\|\mathcal{A}\| \leq \sum_{i=1}^{n}\left\|A_{i}\right\| \Longrightarrow \mathrm{e}^{-c|| \mathcal{A} \|} \prod_{i=1}^{n} \mathrm{e}^{c\left\|A_{i}\right\|} \geq 1
$$

onde $c \in \mathbb{R}^{+}$,

$$
|\rho(\mathcal{A})| \leq 4^{|\mathcal{A}|} \mathrm{e}^{-c|| \mathcal{A}||} \sum_{n=1}^{\infty} \frac{(\beta \epsilon)^{n}}{n !} \sum_{\begin{array}{c}
A_{1}, \cdots, A_{n}: \\
\mathfrak{C}_{n}\left(A_{1}, \cdots, A_{n}\right)=\mathcal{A}
\end{array}} \prod_{i=1}^{n}\left\|T_{A_{i}}\right\| \mathrm{e}^{c\left\|A_{i}\right\|}
$$

também

$$
\sum_{\substack{A_{1}, \cdots, A_{n}: \\ \mathfrak{C}_{n}\left(A_{1}, \cdots, A_{n}\right)=\mathcal{A}}} \prod_{i=1}^{n}\left\|T_{A_{i}}\right\| \mathrm{e}^{c|| A_{i} \|} \leq\left(\sum_{i=1}^{n}\left\|T_{A_{i}}\right\| \mathrm{e}^{c|| A_{i} \|}\right)^{n} \leq\left(|\mathcal{A}| \sup _{x \in \mathbb{Z}^{2}} \sum_{A \ni x}\left\|T_{A}\right\| \mathrm{e}^{c\|A\|}\right)^{n}
$$

aqui $A \subset \mathcal{A}$ e $\|A\|=\min _{B \supset A}|B|$ tal que $B$ é conetado, assim

$$
|\rho(\mathcal{A})| \leq 4^{|\mathcal{A}|} \mathrm{e}^{-c|| \mathcal{A} \|} \sum_{n=1}^{\infty} \frac{\left(\beta \epsilon\|\mathcal{A}\|\|T\|_{c}\right)^{n}}{n !} \leq \mathrm{e}^{-\left(c-\beta \epsilon\|T\|_{c}-\ln 4\right)\|\mathcal{A}\|}
$$

onde

$$
\|T\|_{c}:=\sup _{x \in \mathbb{Z}^{2}} \sum_{A \ni x}\left\|T_{A}\right\| \mathrm{e}^{c\|A\|}
$$

Para um $\epsilon$ dado e $c>\ln 4+\mathcal{O}(\epsilon)$ temos que os pesos $\rho(\mathcal{A}) \rightarrow 0$ se $\|\mathcal{A}\| \rightarrow \infty$ e a condição para a convergência da expansão por clusters (5.34) pode ser satisfeita. Diretamente da formula de Duhamel vemos que

$$
\rho(\mathcal{A})=\sum_{k=0}^{\infty} \mathfrak{c}_{k} \epsilon^{k}
$$


se

$$
\sum_{k=0}^{\infty}\left|\mathfrak{c}_{k}\right| r^{k} \leq \infty
$$

a função $\rho(A)$ sera analítica para $\epsilon \leq r$ (teorema provado por Weierstrass). Assim para $c>\ln 4+\mathcal{O}(\epsilon), \rho(A)$ sera analítica respeito a $\epsilon$ ao existir uma serie majorante para (5.45) que converge.

Fazendo um procedimento similar podemos mostrar também que os pesos $\rho(\mathcal{A})$ vão converge para pertubações sobre o potencial químico local $\mu_{x}$, de (5.30) e (5.31) notamos que

$$
\widetilde{H}\left(\mu_{x}+\delta \mu_{x}\right)=H_{s c}\left(\mathfrak{b}_{1}, \mathfrak{b}_{2}, \mathfrak{b}_{3}, \mathfrak{b}_{4}\right)+\epsilon \mathbf{T}_{f}-\delta \mu_{x}\left(n_{x, \uparrow}+n_{x, \downarrow}\right)
$$

Seja

$$
\epsilon \mathbf{T}_{f}-\delta \mu_{x} \mathbf{n}_{f}=\sum_{A \subset \Lambda}\left(\epsilon T_{A}-\delta \mu_{x} n_{A}\right)=\sum_{A \subset \Lambda} \mathfrak{T}_{A}
$$

onde $\mathbf{n}_{f}=n_{x, \uparrow}+n_{x, \downarrow} \mathrm{e}$

$$
n_{A}=\left\{\begin{array}{ccc}
n_{x_{p, q, \uparrow}}+n_{x_{p, q, \downarrow}} & \text { se } & A=\left\{x_{p, q}\right\} \\
0 & \text { se } & A \neq\left\{x_{p, q}\right\}
\end{array}\right.
$$

aqui $x_{p, q} \in\{x+2 L(p, q) \mid p, q \in \mathbb{N}\}$, e $L$ é tal que o volume de um bloco base vem dado por $\left|\Lambda_{b}\right|=4 L^{2}$.

Para os pesos temos então

$$
\begin{gathered}
\rho(\mathcal{A})=\mathrm{e}^{-\beta p_{0}|\mathcal{A}|} \sum_{n=1}^{\infty}(-1)^{n} \int_{0}^{\beta} d \beta_{1} \int_{0}^{\beta_{1}} d \beta_{2} \cdots \int_{0}^{\beta_{n-1}} d \beta_{n} \\
\sum_{\substack{A_{1}, \cdots, A_{n}: \\
\mathfrak{C}_{n}\left(A_{1}, \cdots, A_{n}\right)=\mathcal{A}}} \operatorname{Tr}_{\mathcal{A}}\left(\mathrm{e}^{-\left(\beta-\beta_{1}\right) H_{0}} \mathfrak{T}_{A_{1}} \mathrm{e}^{-\left(\beta_{1}-\beta_{2}\right) H_{0}} \mathfrak{T}_{A_{2}} \cdots \mathfrak{T}_{A_{n}} \mathrm{e}^{-\beta_{n} H_{0}}\right)
\end{gathered}
$$

observamos que

$$
\left\|\mathrm{e}^{-\left(\beta-\beta_{1}\right) H_{0}} \mathfrak{T}_{A_{1}} \mathrm{e}^{-\left(\beta_{1}-\beta_{2}\right) H_{0}} \mathfrak{T}_{A_{2}} \cdots \mathfrak{T}_{A_{n}} \mathrm{e}^{-\beta_{n} H_{0}}\right\| \leq\left\|\mathrm{e}^{-\beta H_{0}}\right\| \sum_{k=0}^{n} \epsilon^{k}\left(\delta \mu_{x}\right)^{n-k} \prod_{i=1}^{k} \prod_{j=k+1}^{n}\left\|T_{A_{i}}\right\|\left\|n_{A_{j}}\right\|
$$


$\mathrm{Ou}$

$$
\left\|\mathrm{e}^{-\left(\beta-\beta_{1}\right) H_{0}} \mathfrak{T}_{A_{1}} \mathrm{e}^{-\left(\beta_{1}-\beta_{2}\right) H_{0}} \mathfrak{T}_{A_{2}} \cdots \mathfrak{T}_{A_{n}} \mathrm{e}^{-\beta_{n} H_{0}}\right\| \leq \mathrm{e}^{\beta p_{0}|\mathcal{A}|} \sum_{k=0}^{n} \epsilon^{k}\left(2 \delta \mu_{x}\right)^{n-k} \prod_{i=1}^{k}\left\|T_{A_{i}}\right\|
$$

assim similar a (5.43) temos

$$
|\rho(\mathcal{A})| \leq 4^{|\mathcal{A}|} \sum_{n=1}^{\infty} \frac{(\beta)^{n}}{n !} \sum_{k=0}^{n} \epsilon^{k}\left(2 \delta \mu_{x}\right)^{n-k} \sum_{\substack{A_{1}, \cdots, A_{k}: \\ \mathfrak{C}_{n}\left(A_{1}, \cdots, A_{n}\right)=\mathcal{A}}} \prod_{i=1}^{k}\left\|T_{A_{i}}\right\|
$$

ao igual que em (5.44) vemos que

$$
\sum_{\substack{A_{1}, \cdots, A_{k}: \\ \mathfrak{C}_{n}\left(A_{1}, \cdots, A_{n}\right)=\mathcal{A}}} \prod_{i=1}^{k}\left\|T_{A_{i}}\right\| \mathrm{e}^{c|| A_{i} \|} \leq\left(\sum_{i=1}^{k}\left\|T_{A_{i}}\right\| \mathrm{e}^{c\left\|A_{i}\right\|}\right)^{k} \leq\left(|\mathcal{A}| \sup _{x \in \mathbb{Z}^{2}} \sum_{A \ni x}\left\|T_{A}\right\| \mathrm{e}^{c|| A \|}\right)^{k}
$$

$\mathrm{e}$

$$
\begin{aligned}
|\rho(\mathcal{A})| \leq 4^{|\mathcal{A}|} \mathrm{e}^{-c|| \mathcal{A} \|} \sum_{n=1}^{\infty} \frac{(\beta)^{n}}{n !} \sum_{k=0}^{n} \epsilon^{k}\left(2 \delta \mu_{x}\right)^{n-k}\left(|\mathcal{A}|\|T\|_{c}\right)^{k} \\
\leq 4^{|\mathcal{A}|} \mathrm{e}^{-c|| \mathcal{A}||} \sum_{n=1}^{\infty} \frac{\beta^{n}}{n !} \sum_{k=0}^{n} \epsilon^{k}\left(2 \delta \mu_{x}\right)^{n-k}\left(|\mathcal{A}|\|T\|_{c}\right)^{k} \\
\leq 4^{|\mathcal{A}|} \mathrm{e}^{-c|| \mathcal{A}||} \sum_{n=1}^{\infty} \frac{\beta^{n}}{n !} \sum_{k=0}^{n}\left(\begin{array}{c}
n \\
k
\end{array}\right)\left(\epsilon|\mathcal{A}|\|T\|_{c}\right)^{k}\left(2 \delta \mu_{x}\right)^{n-k} \\
\leq 4^{|\mathcal{A}|} \mathrm{e}^{-c|| \mathcal{A}||} \sum_{n=1}^{\infty} \frac{(\beta|\mathcal{A}|)^{n}}{n !}\left(\epsilon\|T\|_{c}+2 \delta \mu_{x}\right)^{n} \\
\leq \mathrm{e}^{-\left(c-\ln 4-\beta\left(\epsilon\|T\|_{c}+2 \delta \mu_{x}\right)\right)\|\mathcal{A}\|} .
\end{aligned}
$$

Vemos que se $c>\ln 4+\mathcal{O}\left(\epsilon+\delta \mu_{x}\right)$ os pesos são convergentes se $|\mathcal{A}| \rightarrow \infty$. Similar a como foi dito anteriormente, observamos diretamente de Duhamel que

$$
\rho(\mathcal{A})=\sum_{k, l=0}^{\infty} \mathfrak{c}_{k, l} \epsilon^{k}(\underbrace{\delta \mu_{x}}_{\tilde{\mu}_{x}-\mu_{x}})^{l}
$$


se

$$
\sum_{k, l=0}^{\infty}\left|\mathfrak{c}_{k, l}\right| r^{k+l} \leq \sum_{k, l=0}^{\infty}\left|\mathfrak{d}_{k+l}\right| r^{k+l}=\sum_{K=0}^{\infty}(K+1)\left|\mathfrak{d}_{K}\right| r^{K}<\infty
$$

$\rho(\mathcal{A})$ é analítica para $\left(\epsilon, \delta \mu_{x}\right) \leq \max \left(\epsilon, \delta \mu_{x}\right) \leq r$.

Assim é claro de $(5.46)$ que os pesos $\rho(\mathcal{A})$ são analíticos respeito aos parâmetros $\left(\mu_{x}\right.$, $\epsilon)$, em particular perto do ponto $\left(\mu_{x}, 0\right)$, por o que a expansão (5.41) também sera. Para $\epsilon \rightarrow 0$

$$
\tilde{p}_{\Lambda}\left(\mu, U_{0}, \hat{w}, \epsilon, \beta\right) \approx \underbrace{\tilde{p}_{\Lambda}\left(\mu, U_{0}, \hat{w}, 0, \beta\right)}_{p_{0}}+\epsilon \partial_{\epsilon} \tilde{p}_{\Lambda}\left(\mu, U_{0}, \hat{w}, \epsilon, \beta\right)
$$

$\mathrm{e}$

$$
\partial_{\mu_{x}} \tilde{p}_{\Lambda}\left(\mu, U_{0}, \hat{w}, \epsilon, \beta\right) \approx \partial_{\mu_{x}} p_{0}+\epsilon \partial_{\mu_{x}} \partial_{\epsilon} \tilde{p}_{\Lambda}\left(\mu, U_{0}, \hat{w}, \epsilon, \beta\right)=\partial_{\mu_{x}} p_{0}+\mathcal{O}(\epsilon)
$$

Vemos assim que o valor médio da densidade eletrônica local

$$
\frac{\left\langle n_{x, \uparrow}+n_{x, \downarrow}\right\rangle}{\left|\Lambda_{b}\right|}=\partial_{\mu_{x}} \tilde{p}_{\Lambda}\left(\mu, U_{0}, \hat{w}, \epsilon, \beta\right)
$$

se desloca pouco da densidade eletrônica sem "perturbação" sempre que o parâmetro de hopping seja suficientemente pequeno. Esto prova que as ondas de densidade são estáveis ao considerar o efeito de um termo cinético perturbativo.

Adicionalmente, observamos que o cálculo explicito da densidade local pode ser realizado com auxilio de uma extensão de teorema de expansão por clusters para as chamadas funções de correlação, dado em [33]. Seja

$$
\mathcal{Z}\left(A_{1}, \cdots, A_{m}\right)=\sum_{n \geq m} \frac{1}{(n-m) !} \int d \mu\left(A_{m}+1\right) \cdots \int d \mu\left(A_{n}\right) \prod_{1 \leq i<j \leq n}\left(1+\zeta\left(A_{i}, A_{j}\right)\right)
$$

e

$$
\hat{\mathcal{Z}}\left(A_{1}, \cdots, A_{m}\right)=\sum_{n \geq m} \frac{n !}{(n-m) !} \int d \mu\left(A_{m}+1\right) \cdots \int d \mu\left(A_{n}\right) \varphi\left(A_{1}, \cdots, A_{n}\right) .
$$


Em [33] é provado que

$$
\frac{\mathcal{Z}\left(A_{1}, \cdots, A_{m}\right)}{\mathcal{Z}}=\sum_{\left\{V_{1}, \cdots, V_{k}\right\}} \prod_{j=1}^{k} \hat{Z}\left(\left(A_{i}\right)_{i \in V_{j}}\right)
$$

onde a soma é sobre as partições de $\{1, \cdots, m\}$, ou seja $V_{i} \cup \cdots \cup V_{k}=\{1, \cdots, m\}$ com $V_{i} \cap V_{j}=\emptyset$ se $i \neq j$.

Em nosso caso notamos que a densidade num ponto fixo $x$ qualquer pode ser escrita em termos das quantidades

$$
\frac{\mathcal{Z}\left(\mathcal{A}_{1}\right)}{\mathcal{Z}}
$$

\section{Perspectivas}

Na continuação deste trabalho pode estudar-se mais detalhadamente o limite de temperatura nula, através de melhores estimativas das atividades dos polímeros ou através do desenvolvimento de métodos perturbativos para baixas temperatura, tais como "contour expansions".

O caso de pares fermiônicos com spin 1 também seria de interesse explorar, para os quais pode haver ferromagnetismo coletivo.

Pode estudar-se também métodos perturbativos para tratar o caso de bipolarons não localizados, como foi feito com auxilio de cálculo numérico para o problema de três corpos [39]. Para o problema coletivo não há, neste caso, invariância por permutação de blocos para o regime de acoplamento forte. 


\section{Bibliografia}

[1] Bonn D. A., Are high-temperature superconductors exotic?. Nat. Physics, (2006)

[2] J. Ranninger, The polaron scenario for high- $T_{c}$ superconductors. Cambridge University Press, (1995)

[3] N. N. Bogoliubov Jr., On Model Dynamical Systems in Statistical Mechanics. Physica, (1966)

[4] Bru, J.-B. and W. Pedra, Effect of a Locally Repulsive Interaction on s-wave Supercondctors. World Scientific, (2010)

[5] Hubbard, J., Electron correlations in narrow energy bands. Journal of The Royal Society, (1963)

[6] Reznik D., Giant electron-phonon anomaly in doped $\mathrm{La}_{2} \mathrm{CuO}_{4}$ and others cuprates. Adv. Condens. Matter Phys, (2010)

[7] Ishihara S, Egami T and Tachiki, Electron-lattice interaction in cuprates: effect of electron correlation. Phys. Rev, (1997)

[8] V.V. Kabanov and D. Mihailovic, Finite-Wave-Vector Phonon Coupling to Degenerate Electronic States in $\mathrm{La}_{2-x} \mathrm{Ba}_{x} \mathrm{CuO}_{4}$. J. Supercond., (2000)

[9] D. Mihailovic D and V.V. Kabanov, Finite wave vector Jahn-Teller pairing and superconductivity in the cuprates. Phys. Rev. B, (2001)

[10] B.I. Kochelaev et al., Three-Spin-Polarons and Their Elastic Interaction in Cuprates. Mod. Phys. Lett. B, (2003) 
[11] K.A. Müller, On the superconductivity in hole doped cuprates. J. Phys.: Condens. Matter, (2007)

[12] N. Plakida, High-Temperature Cuprate Superconductors, Experiment, Theory and Applications. Springer-Verlag, (2010)

[13] R. Wesche, Physical Properties of High-Temperature Superconductors. Wiley series in materials for electronic and optoelectronic applications, (2015)

[14] W.J. Padilla et al., Constant effective mass across the phase diagram of high$T_{c}$ cuprates. Phys. Rev. B, (2005)

[15] W. Chaibi et al., Effect of a magnetic field in photodetachment microscopy. Eur. Phys. J. D, (2010)

[16] J. Mannhart and H. Hilgenkamp, Interfaces involving complex superconductors. Phys. C, (1999)

[17] G.F. Giuliani and G. Vignale, Quantum Theory of the Electron Liquid. Cambrigde Univ. Press, (2005)

[18] M.A. Kastner, R.J. Birgeneau, G. Shirane and Y. Endoh, Magnetic transport and optical properties of monolayer copper oxides. Rev. Mod. Phys, (1998)

[19] E.H. Lieb, N. Rougerie and J. Yngvason, Local incompressibility estimates for the laughlin phase. arXiv:1701.09064, (2017)

[20] D. Reagor, Large Dielectric Constants and Massive Carriers in $\mathrm{La}_{2} \mathrm{CuO}_{4}$. Phys. Rev. Lett., (1989)

[21] C.Y. Chen et al., Frequency and magnetic-field dependence of the dielectric constant and conductivity of $\mathrm{La}_{2} \mathrm{CuO}_{4+y}$. Phys. Rev. B, (1991)

[22] I. Božović, X. He, J. Wu and A.T. Bollinger, Dependence of the critical temperature in overdoped copper oxides on superfluid density. Nature, (2016) 
[23] B.J. Ramshaw et al., Broken rotational symmetry on the Fermi surface of a high$\mathrm{T}_{c}$ superconductor. Quantum Materials, (2017)

[24] K.A. Müller and H. Keller, High-Tc Superconductivity 1996: Ten Years After the Discovery. NATO ASI Ser. E, (1996)

[25] R. Kasanov et al., Experimental Evidence for Two Gaps in the High-Temperature $\mathrm{La}_{1.83} \mathrm{Sr}_{0.17} \mathrm{CuO}_{4}$ Superconductor. Phys. Rev. Lett., (2007)

[26] A. Furrer, Admixture of an s-Wave Component to the d-Wave Gap Symmetry in High-Temperature Superconductors. J. Supercond. Nov. Magn., (2008)

[27] T. Timusk and B. Statt, The pseudogap in high-temperature superconductors: an experimental survey. Rep. Prog. Phys., (1999)

[28] L. Pintschovius, D. Reznik and K. Yamada, Oxygen phonon branches in overdoped $\mathrm{La}_{1.7} \mathrm{Sr}_{0.3} \mathrm{CuO}_{4}$. Phys. Rev. B, (2006)

[29] J.-B. Bru, W. de Siqueira Pedra and A. Delgado de Pasquale, d-Wave pairing driven by bipolaric modes related to giant electron-phonon anomalies in high- $T_{c}$ superconductors. J. Stat. Mech., (2015)

[30] Y. Marnoe et al., Evaluation of Spin-Triplet Superconductivity in $\mathrm{Sr}_{2} \mathrm{RuO}_{4}$. J. Phys. Soc. Jpn., (2012)

[31] H. Araki and H. Moriya, Equilibrium statistical mechanics of fermion lattice systems. Rev. Math. Phys., (2003)

[32] R. Kotecký and D. Preiss, Cluster expansion for abstract polymer models. Commun. Math. Phys., (1986)

[33] D. Ueltschi, Cluster Expansions and Correlation Functions. Moscow Mathematical Journal, (2004)

[34] D. Ueltschi, Analyticity in Hubbard Models. Journal of Statistical Physics, (1999) 
[35] Reed, M., Simon, B., Methods of Modern Mathematical Physics, Vol. IV. Academic Press, New York-London, (1972)

[36] Teschl, G., Mathematical Methods in Quantum Mechanics. American Mathematical Society, (2009)

[37] Barut, A. O., Theory of Group Representations and Applications. World Scientific, (1986)

[38] Handbook of High-Temperature Superconductor Electronics. Edited by Neeraj Khare, (2005)

[39] Bru, J.-B., W. Pedra and A. Delgado, Isotropic Bipolaron-Fermion-Exchange Theory and Unconventional Pairing in Cuprate Superconductors, (To appear)

[40] M. Imada, A. Fujimori and Y. Tokura, Metal-insulator transitions. Rev. Mod. Phys., (1998)

[41] B. Keimer et al., From quantum matter to high-temperature superconductivity in copper oxides. Nature, (2015)

[42] K.A. Müller and J.G. Bednorz, Possible high $T_{c}$ superconductivity in the Ba-La-Cu-O system. Z. Phys. B., (1986)

[43] A.K. Saxena, High-Temperature Superconductors. Springer-Verlag, Berlin Heidelberg, (2010)

[44] C.C. Tsuei and J.R. Kirtley, Pairing symmetry in cuprate superconductors. Rev. Mod. Phys., (2000)

[45] H. Keller, A. Bussmann-Holder and K.A. Müller, Jahn-Teller physics and high- $T_{c}$ superconductivity. Materials Today, (2008)

[46] K.A. Müller, On the macroscopic $s$ - and $d$-wave symmetry in cuprate superconductors. Phil. Mag. Lett., (2002) 